\title{
Promoting cardiovascular health through personalized supervised exercise therapy in intermittent claudication
}

Citation for published version (APA):

Jansen, S. C. P. (2022). Promoting cardiovascular health through personalized supervised exercise therapy in intermittent claudication. [Doctoral Thesis, Maastricht University]. Proefschriftenprinten.nl. https://doi.org/10.26481/dis.20220211sj

Document status and date:

Published: 01/01/2022

DOI:

$10.26481 /$ dis.20220211sj

Document Version:

Publisher's PDF, also known as Version of record

\section{Please check the document version of this publication:}

- A submitted manuscript is the version of the article upon submission and before peer-review. There can be important differences between the submitted version and the official published version of record. People interested in the research are advised to contact the author for the final version of the publication, or visit the DOI to the publisher's website.

- The final author version and the galley proof are versions of the publication after peer review.

- The final published version features the final layout of the paper including the volume, issue and page numbers.

Link to publication

\footnotetext{
General rights rights.

- You may freely distribute the URL identifying the publication in the public portal. please follow below link for the End User Agreement:

www.umlib.nl/taverne-license

Take down policy

If you believe that this document breaches copyright please contact us at:

repository@maastrichtuniversity.nl

providing details and we will investigate your claim.
}

Copyright and moral rights for the publications made accessible in the public portal are retained by the authors and/or other copyright owners and it is a condition of accessing publications that users recognise and abide by the legal requirements associated with these

- Users may download and print one copy of any publication from the public portal for the purpose of private study or research.

- You may not further distribute the material or use it for any profit-making activity or commercial gain

If the publication is distributed under the terms of Article 25fa of the Dutch Copyright Act, indicated by the "Taverne" license above, 


\section{PROMOTING CARDIOVASCULAR HEALTH through personalized supervised exercise therapy in intermittent claudication}





\section{PROMOTING CARDIOVASCULAR HEALTH}

through personalized supervised exercise therapy in intermittent claudication

Sandra Catharina Petronella Jansen 


Printing of this thesis was financially supported by Maastricht University, Catharina Hospital Eindhoven, and Chronisch ZorgNet.

\section{Maastricht University}

\section{(2) catharina \\ een santeon ziekenhuis}

Chronisch ZorgNet

bewegen \& leefstijl

\section{COLOFON}

Author: Sandra Catharina Petronella Jansen

Printing: Proefschriften.nl, Ede

Layout and design: Sara Terwisscha van Scheltinga, persoonlijkproefschrift.nl

ISBN: 978-94-6421-608-0

\section{(C) S.C.P. Jansen, 2022}

All rights reserved. No part of this publication may be reproduced or transmitted, in any form by any means, without permission of the author. 


\title{
Promoting cardiovascular health through personalized supervised exercise therapy in intermittent claudication
}

\author{
Proefschrift \\ ter verkrijging van de graad van doctor aan de Universiteit Maastricht, \\ op gezag van de Rector Magnificus, Prof. dr. Pamela Habibovic, \\ volgens het besluit van het College van Decanen, \\ in het openbaar te verdedigen \\ op vrijdag 11 februari 2022 om 16.00 uur \\ door \\ Sandra Catharina Petronella Jansen \\ geboren te Veldhoven op 19 februari 1992
}




\section{Promotor}

Prof. dr. J.A.W. Teijink

\section{Copromotor}

Dr. M.R.M. Scheltinga (Máxima Medisch Centrum)

Dr. E.V. Rouwet (Erasmus Medisch Centrum)

\section{Beoordelingscommissie}

Prof. dr. M.H. Prins (voorzitter)

Dr. M.E.L. Bartelink (UMC Utrecht)

Prof. dr. H. ten Cate

Dr. B.H.P. Elsman (Deventer Ziekenhuis) 


\section{TABLE OF CONTENTS}

Part I

Introduction

9

Chapter 1

General introduction and outline of thesis

Part II

Studies on the potential influence of supervised exercise

21 therapy on cardiovascular risk, secondary interventions and mortality

Chapter 2

A systematic review and meta-analysis of the effects of supervised exercise therapy on modifiable cardiovascular risk factors in intermittent claudication

Chapter 3

Successful implementation of the exercise first approach for intermittent claudication in the Netherlands is associated with few lower limb revascularisations

\section{Part III Suitability of supervised exercise therapy in patients with intermittent claudication \\ Chapter 4 \\ Protocol for a prospective, longitudinal cohort study on the effect of arterial disease level on the outcomes of supervised exercise in intermittent claudication: the ELECT Registry \\ Chapter 5 The effect of arterial disease level on outcomes of supervised exercise therapy for intermittent claudication: a prospective cohort study}

Chapter 6

Supervised exercise therapy is effective for patients with intermittent claudication regardless of psychological constructs

Chapter $7 \quad$ Modes of exercise training for intermittent claudication

Part IV

Summary and discussion

Chapter 8

Summary and general discussion

Chapter 9

Impactparagraaf

Part V

Postscript

Chapter 10

Dankwoord

Lijst van publicaties

256

Curriculum vitae 


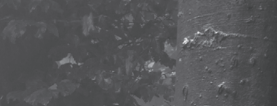

is setwe

$x=\ldots+\infty$

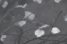

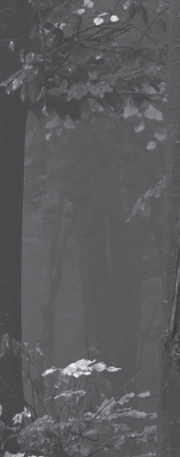

Heste

2

fordegis?

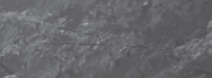

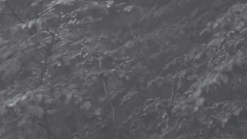
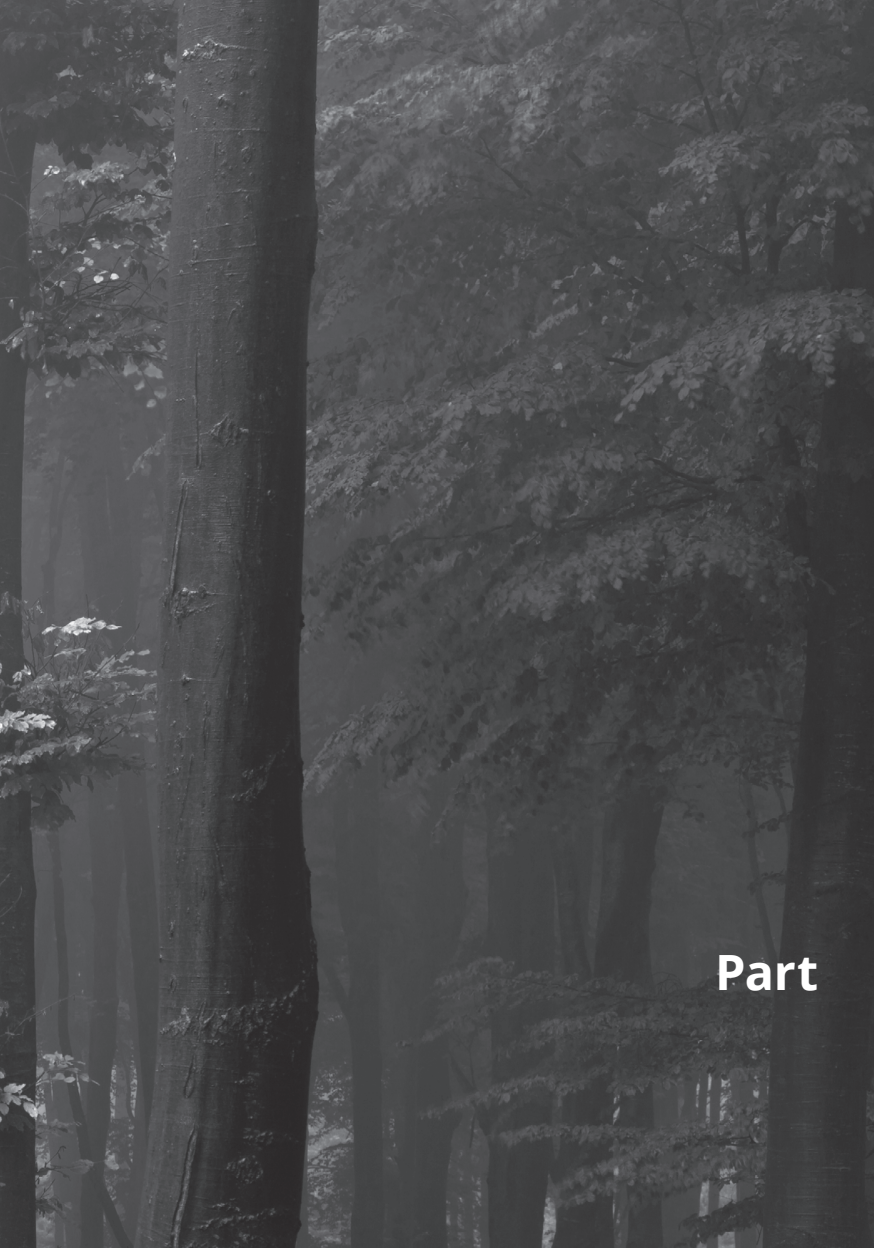

8

19.

Part

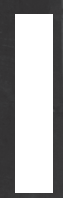

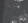

$a^{2}$

The

4

(1)

18

$1: 5$

ti
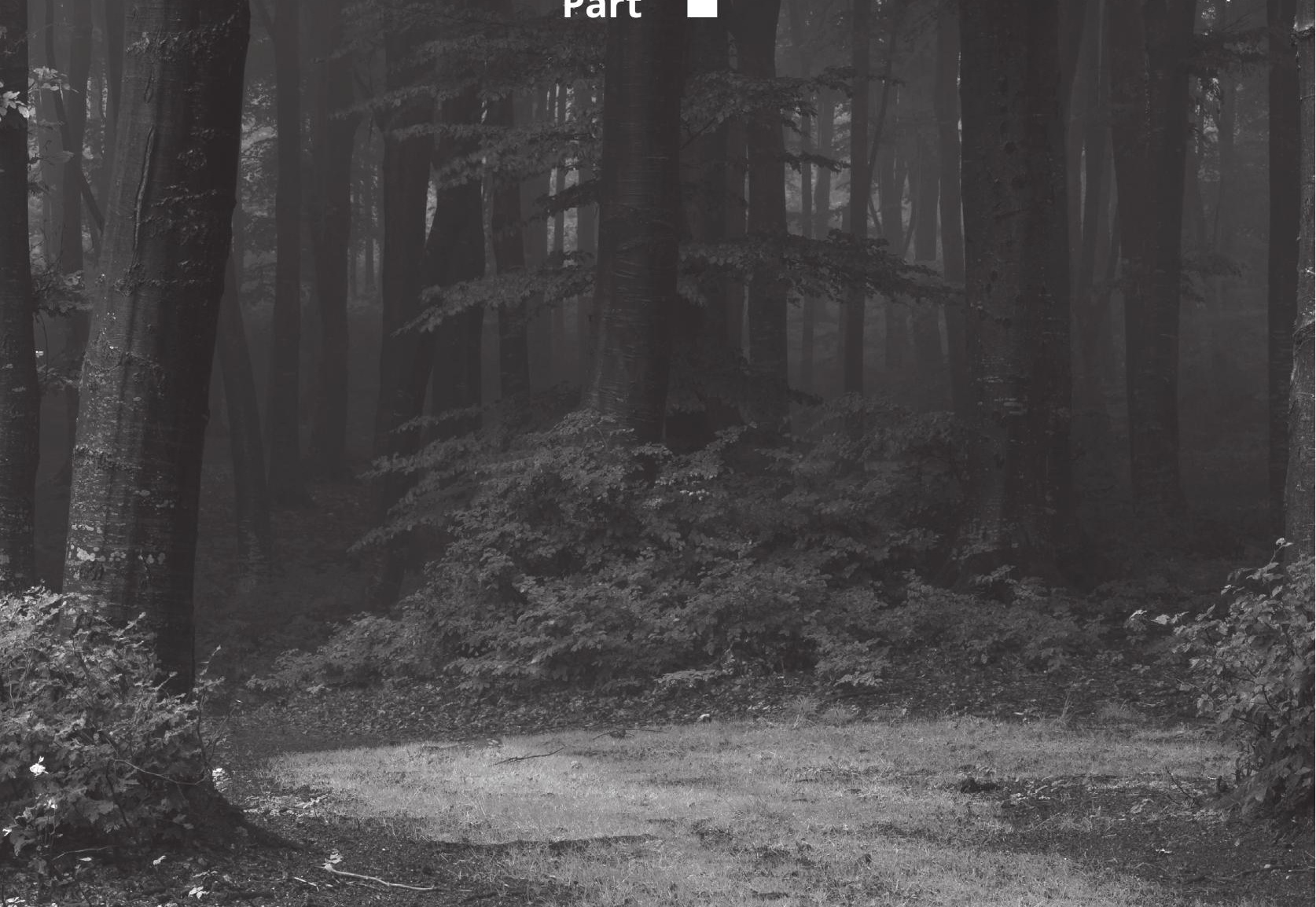


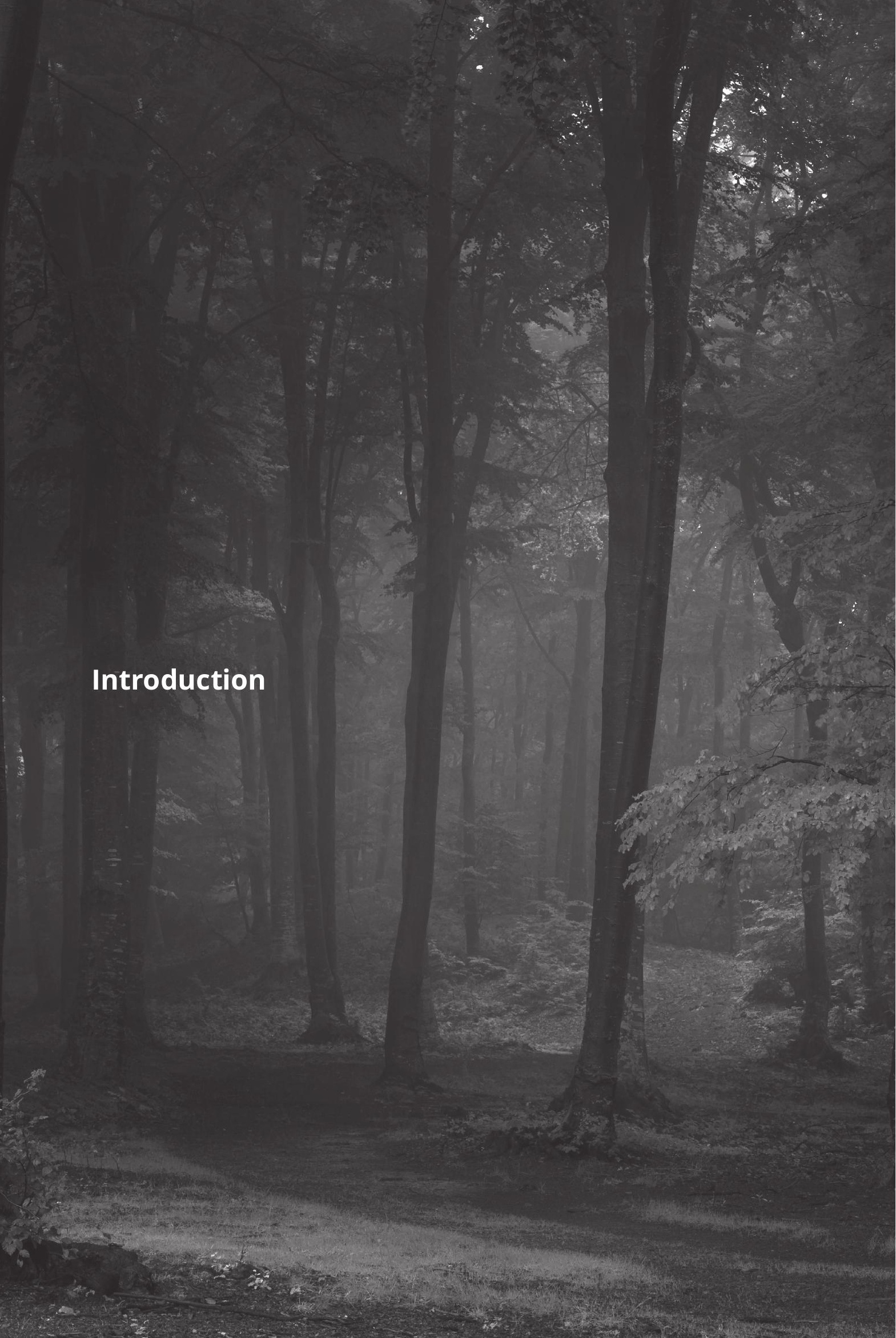





\section{Chapter}

\section{General introduction and outline of thesis}

\section{Adapted from}

Teijink, J. \& Jansen, S. Hoofdstuk 12: Beweging.

De Vries, M., \& de Weijer, T. (Red.) Handboek Leefstijlgeneeskunde (pp. 221-237).

Houten, Nederland: Bohn Stafleu van Loghum. 2020

https://doi.org/10.1007/9789036823241 
Lower extremity peripheral artery disease (PAD) is an expression of systemic atherosclerosis affecting the infrarenal aorta and lower limb arteries. Its prevalence is highly age-related, as it usually occurs after the age of 50 and affects an estimated 20 percent of 80 -year-old patients. ${ }^{1}$ The majority of patients is asymptomatic or has atypical leg symptoms. Approximately 20-30 percent presents with the classical symptoms of intermittent claudication (IC), defined as a cramping pain in calf, thigh or buttock muscles during walking that is relieved upon a short period of rest. Although these symptoms can be very invalidating, the larger issue is the associated increased risk of cardiovascular events. ${ }^{2,3}$ Approximately $10-15 \%$ of PAD patients will die within 5 years after the onset of IC symptoms, with fatal cardiovascular events accounting for $70 \%$ of these deaths. An additional 30\% of patients are confronted with a non-fatal cardiovascular event. ${ }^{1,4-6}$ Current guidelines advice to start supervised exercise therapy (SET) in patients presenting with IC., However, the majority of studies on SET focus on outcomes such as walking distance and quality of life, but relatively few data are available on the potential effects of SET on cardiovascular outcomes such as modifiable risk factors and mortality.

The two main components of IC treatment are cardiovascular risk management and symptom relief. SET and endovascular revascularisation (ER) have been shown to be equally effective in improving walking capacity and health-related quality of life..$^{8,9}$ However, SET is non-invasive and more cost-effective..$^{10,11}$ Therefore, current guidelines recommend SET as initial treatment for IC and support a stepped care approach in which revascularization is reserved once SET is unsuccessful. ${ }^{1,7}$ Occasionally however, revascularisation can be proposed as primary treatment, alongside with SET, if daily life activity is severely compromised. ${ }^{1,7,12}$ Unfortunately, use of this stepped care model is not yet self-evident as implementation is hampered by suboptimal availability of SET facilities, referral patterns, and the lack of reimbursement of SET in most countries. ${ }^{13,14}$

In 2011, already $56 \%$ of Dutch IC patients received SET as the primary treatment modality. A $22 \%$ increase, compared to the year 2009, was associated with decreased vascular intervention rates and $6 \%$ lower average costs of IC treatment per patient. ${ }^{11,15}$ There are no recent data available on the consequences of the 'exercise-first approach' in the Netherlands or whether this affects the number of secondary vascular interventions in the long term.

Despite these well-founded recommendations, supposed arguments for an intervention-first strategy remain. ${ }^{14,16}$ Some vascular professionals argue that SET will probably fail in certain subsets of patients. Such claims are mostly practicebased as the current literature provides no grounds for the identification of a subset of patients who will be unresponsive to SET. Aortoiliac disease location is a common argument for such an early revascularisation. ${ }^{17}$ The idea that patients with proximal disease would benefit less from SET compared to patients with distal lesions remains widespread, despite the lack of empirical evidence. ${ }^{8,18,19}$ Therefore, 
the demand for research investigating the effect of the location of stenosis on the effectiveness of SET in a real-world setting is highly relevant.

Other factors possibly affecting SET's effectiveness are psychosocial, which may influence treatment outcomes as they act as potential barriers to treatment adherence. ${ }^{20}$ For example, it may seem that depression and type D personality, which is related to neuroticism and introversion, negatively influence walking ability and quality of life. ${ }^{21,22}$ If certain psychological constructs indeed affect outcomes, IC treatment may require a tailored approach in these patients. However, research on the potential moderating influence of certain psychological constructs on SET outcomes is scarce.

Essentially, SET is a physical rehabilitation program that revolves around lifestyle interventions. Its main goal is to increase physical capacity through exercise training which enables patients to be sufficiently physically active in a safe and comfortable way despite their chronic disease (see box for explanation of the terms exercise and physical activity). Physical activity is a key factor in aetiology, prevention and treatment of cardiovascular risk. ${ }^{23-26}$ Moreover, physical activity is an actual treatment modality to reduce symptoms and improve outcomes, not only for PAD but also for common comorbidities (see table 1). ${ }^{23-37}$ Although the evidence for the claimed beneficial effects is abundant in various settings, little is known about the effects of SET on cardiovascular risk in the IC population. Furthermore, it is not clear if SET contributes to actual change of health behaviour, for example via increasing self-efficacy. Self-efficacy is an important mediator of behaviour change and can be promoted through mastery experiences, social modeling, and motivational interviewing. ${ }^{38,39}$ As these are main components of SET, it would be interesting to evaluate the potential effect of SET on self-efficacy.

Ideally, exercise therapy is supervised by qualified personnel. ${ }^{40}$ SET typically contains up to 37 individual sessions of 30 minutes during a 3 to 12 months period. The main part of the program consists of treadmill-based or track-based exercise until the patient experiences claudication symptoms of moderate severity. This 'exercise-rest-exercise' cycle is repeated several times per session. ${ }^{41,42}$ As walking may not be the optimal mode of exercise for all patients, alternatives such as cycling or resistance training are potentially interesting. However, it is not yet clear if such alternative modes produce equivalent results compared to walking-based SET. ${ }^{43}$

Exercise is a subset of physical activity that is planned, structured, and repetitive and has the objective to improve or maintain physical fitness.

Physical activity is defined as 'any bodily movement produced by skeletal muscles that results in energy expenditure' and also includes activities related to work, housekeeping, leisure time or transportation. 
Table 1 - Summary of positive effects of physical activity

\begin{tabular}{lll}
\hline modifiable risk factors & $\downarrow$ systolic and diastolic blood & $\downarrow$ visceral adipose tissue \\
& pressure & $\uparrow$ exercise tolerance and \\
& $\downarrow$ LDL-and non-HDL & capacity \\
& cholesterol & $\uparrow$ muscle mass and strength \\
& $\downarrow$ insulin resistance & $\uparrow$ compliance to lifestyle \\
& $\uparrow$ glucose uptake & regimens \\
& $\downarrow$ risk of myocardial ischemia and arrythmia \\
ischemic heart disease & fewer and shorter hospitalisations \\
and heart failure & $\downarrow$ cardiovascular mortality (reduction up to 30 percent) \\
& peripheral artery disease & $\uparrow$ functional and maximum walking distance \\
diabetes mellitus & $\uparrow$ glycaemic control \\
(type 1 and 2) & $\downarrow$ cardiovascular and overall mortality \\
obesity & improved body composition \\
& positive effect on maintaining weight \\
cancer & $\downarrow$ risk on breast, colon and lung cancer \\
& accelerated recovery and fewer complications after treatment \\
chronic obstructive & $\downarrow$ exercise-induced dyspnoea \\
pulmonary disease & accelerated recovery after acute exacerbation \\
& $\downarrow$ health care use \\
& $\downarrow$ mortality \\
general & $\uparrow$ quality of life \\
& improved mental health \\
& helps maintain independence in daily life activities \\
& $\downarrow$ fall risk and related fractures \\
& positive effect on cognitive impairment \\
\hline
\end{tabular}

\section{General aim of thesis}

This thesis focuses on optimising supervised exercise therapy (SET) with emphasis on personalisation of treatment and cardiovascular risk management in patients with intermittent claudication (IC).

\section{Specific aims}

1. To analyse the effects of SET on modifiable cardiovascular risk factors in IC.

2. To determine whether primary treatment (SET, endovascular revascularisation or open surgery) is related to secondary lower limb revascularisation rates and survival in patients with IC.

3. To assess if level of arterial obstruction determines the effectiveness of SET in IC.

4. To determine possible associations between a set of psychological constructs and treatment outcomes in IC.

5. To investigate whether self-efficacy increased after SET in patients with IC.

6. To evaluate the effects of alternative modes of SET compared to traditional walking exercise in patients with IC. 


\section{Outline of thesis}

The first part determines the effects of SET on cardiovascular risk, secondary interventions and mortality. There is growing evidence on the positive effects of exercise on modifiable cardiovascular risk factors, but specific data for SET and IC are scarce. Chapter $\mathbf{2}$ is a systematic review and meta-analysis on the possible effects of SET on such factors, including blood pressure, heart rate, lipid profile, glucose, body mass index, and cigarette smoking. It is unknown which percentage of IC patients is currently receiving SET as primary treatment and whether potentially increased rates have long-term consequences regarding percentages of secondary vascular interventions and mortality. Chapter 3 presents the results of a nationwide retrospective data analysis of health insurance claims regarding IC. It maps recent data on the implementation of the exercise first approach in the Netherlands and assesses the risk of secondary lower limb revascularisations and overall five year survival according to the primary treatment (SET, ER or open surgery).

The second part of this thesis evaluates if SET is suitable for each patient with IC. Some vascular surgeons believe that aortoiliac stenotic disease requires primary revascularisation at all times. Although this assumption is not confirmed in multiple $\operatorname{RCT}^{\prime} s^{8,18,19}$, there is a widely held belief that patients with aortoiliac disease would benefit less from SET in real-world settings. Chapter $\mathbf{4}$ describes the protocol of the ELECT Registry, a prospective longitudinal cohort study recruiting patients with IC receiving primary SET. The main objective was to evaluate whether level of arterial obstruction determines the effectiveness of SET in IC patients (chapter 5). Another possible issue for questioning the suitability of SET is the role of psychological factors on treatment effectiveness. It is thought that depression and type D personality, which is related to neuroticism and introversion, may negatively influence walking ability and quality of life in IC patients. ${ }^{21,22}$ On the other hand, positive factors such as optimism and self-control could contribute to a beneficial outcome. ${ }^{44,45}$ Chapter 6 illustrates a substudy of the ELECT Registry on possible associations between a set of psychological constructs, including depression and anxiety, and SET related outcomes. This study also investigated whether self-efficacy increased during 12 months of SET. Furthermore, walking might not be the most convenient mode of exercise for all patients, especially due to concomitant comorbidities. Chapter $\mathbf{7}$ is a systematic review and meta-analysis determining the effects of alternative modes of SET compared to traditional walking exercise in patients with IC.

This thesis concludes by summarizing and discussing the main findings in chapter 8, also providing future perspectives for clinical practice and research. 


\section{REFERENCES}

1. Aboyans V, Ricco JB, Bartelink MEL, et al. ESC Guidelines on the Diagnosis and Treatment of Peripheral Arterial Diseases, in collaboration with the European Society for Vascular Surgery (ESVS). Eur J Vasc Endovasc Surg. 2017; 10.1016/j.ejvs.2017.07.018.

2. Golomb BA, Dang TT, Criqui $\mathrm{MH}$. Peripheral arterial disease: morbidity and mortality implications. Circulation. 2006;114(7):688-699.

3. Criqui MH, Aboyans V. Epidemiology of peripheral artery disease. Circ Res. 2015;116(9):1509-1526.

4. Criqui $\mathrm{MH}$, Langer RD, Fronek $\mathrm{A}$, et al. Mortality over a period of 10 years in patients with peripheral arterial disease. N Engl J Med. 1992;326(6):381-386.

5. Weitz JI, Byrne J, Clagett GP, et al. Diagnosis and treatment of chronic arterial insufficiency of the lower extremities: a critical review. Circulation. 1996;94(11):3026-3049.

6. Leng GC, Lee AJ, Fowkes FG, et al. Incidence, natural history and cardiovascular events in symptomatic and asymptomatic peripheral arterial disease in the general population. Int J Epidemiol. 1996;25(6):1172-1181.

7. Gerhard-Herman MD, Gornik HL, Barrett C, et al. 2016 AHA/ACC Guideline on the Management of Patients With Lower Extremity Peripheral Artery Disease: Executive Summary: A Report of the American College of Cardiology/American Heart Association Task Force on Clinical Practice Guidelines. Circulation. 2017;135(12):e686-e725.

8. Fakhry F, Rouwet EV, den Hoed PT, Hunink MG, Spronk S. Long-term clinical effectiveness of supervised exercise therapy versus endovascular revascularization for intermittent claudication from a randomized clinical trial. BrJ Surg. 2013;100(9):1164-1171.

9. Fakhry F, Fokkenrood HJ, Spronk S, Teijink JA, Rouwet EV, Hunink MGM. Endovascular revascularisation versus conservative management for intermittent claudication. Cochrane Database Syst Rev. 2018;3:CD010512.

10. van den Houten MM, Lauret GJ, Fakhry F, et al. Cost-effectiveness of supervised exercise therapy compared with endovascular revascularization for intermittent claudication. Br J Surg. 2016;103(12):1616-1625.

11. Hageman D, Fokkenrood HJP, Essers PPM, et al. Improved Adherence to a Stepped-care Model Reduces Costs of Intermittent Claudication Treatment in The Netherlands. EurJ Vasc Endovasc Surg. 2017; 54(1):51-57.

12. Layden J, Michaels J, Bermingham S, Higgins B, Guideline Development G. Diagnosis and management of lower limb peripheral arterial disease: summary of NICE guidance. BMJ. 2012;345:e4947.

13. Popplewell MA, Bradbury AW. Why do health systems not fund supervised exercise programmes for intermittent claudication? Eur J Vasc Endovasc Surg. 2014;48(6):608-610.

14. Gommans LN, Teijink JA. Attitudes to supervised exercise therapy. Br J Surg. 2015;102(10):1153-1155.

15. Fokkenrood HJ, Scheltinga MR, Koelemay MJ, et al. Significant savings with a stepped care model for treatment of patients with intermittent claudication. Eur J Vasc Endovasc Surg. 2014;48(4):423429. 
16. Hageman D, Lauret GJ, Gommans LNM, et al. Supervised Exercise Therapy for Intermittent Claudication Is Increasingly Endorsed by Dutch Vascular Surgeons. Ann Vasc Surg. 2018;47:149-156.

17. van Zitteren $M$, Vriens $P W$, Burger $\mathrm{DH}$, et al. Determinants of invasive treatment in lower extremity peripheral arterial disease. J Vasc Surg. 2014;59(2):400-408 e402.

18. Murphy TP, Cutlip DE, Regensteiner $J G$, et al. Supervised exercise versus primary stenting for claudication resulting from aortoiliac peripheral artery disease: six-month outcomes from the claudication: exercise versus endoluminal revascularization (CLEVER) study. Circulation. 2012;125(1):130-139.

19. Frans FA, Bipat $S$, Reekers JA, Legemate DA, Koelemay MJ, Collaborators SS. SUPERvised exercise therapy or immediate PTA for intermittent claudication in patients with an iliac artery obstruction--a multicentre randomised controlled trial; SUPER study design and rationale. Eur J Vasc Endovasc Surg. 2012;43(4):466-471.

20. Piepoli MF, Hoes AW, Agewall S, et al. 2016 European Guidelines on cardiovascular disease prevention in clinical practice: The Sixth Joint Task Force of the European Society of Cardiology and Other Societies on Cardiovascular Disease Prevention in Clinical Practice (constituted by representatives of 10 societies and by invited experts)Developed with the special contribution of the European Association for Cardiovascular Prevention \& Rehabilitation (EACPR). Eur Heart J. 2016;37(29):2315-2381.

21. Brostow DP, Petrik ML, Starosta AJ, Waldo SW. Depression in patients with peripheral arterial disease: A systematic review. Eur J Cardiovasc Nurs. 2017;16(3):181-193.
22. Sliwka A, Furgal $M$, Maga $P$, et al. The role of psychopathology in perceiving, reporting and treating intermittent claudication: a systematic review. Int Angiol. 2018;37(5):335-345.

23. Eckel RH, Jakicic JM, Ard JD, et al. 2013 AHA/ACC guideline on lifestyle management to reduce cardiovascular risk: a report of the American College of Cardiology/American Heart Association Task Force on Practice Guidelines. J Am Coll Cardiol. 2014;63(25 Pt B):2960-2984.

24. Perk J, De Backer G, Gohlke H, et al. European Guidelines on cardiovascular disease prevention in clinical practice (version 2012). The Fifth Joint Task Force of the European Society of Cardiology and Other Societies on Cardiovascular Disease Prevention in Clinical Practice (constituted by representatives of nine societies and by invited experts). Eur Heart J. 2012;33(13):1635-1701.

25. Piercy KL, Troiano RP, Ballard RM, et al. The Physical Activity Guidelines for Americans. JAMA. 2018;320(19):20202028.

26. Bull FC, Al-Ansari SS, Biddle $S$, et al. World Health Organization 2020 guidelines on physical activity and sedentary behaviour. $\mathrm{Br} J$ Sports Med. 2020;54(24):1451-1462.

27. Pedersen BK, Saltin B. Exercise as medicine - evidence for prescribing exercise as therapy in 26 different chronic diseases. Scand J Med Sci Sports. 2015;25 Suppl 3:1-72.

28. Kujala UM. Evidence on the effects of exercise therapy in the treatment of chronic disease. $\mathrm{Br} J$ Sports Med. 2009;43(8):550-555. 
29. Lee IM, Shiroma EJ, Lobelo F, et al. Effect of physical inactivity on major noncommunicable diseases worldwide: an analysis of burden of disease and life expectancy. Lancet. 2012;380(9838):219-229.

30. Mora S, Cook N, Buring JE, Ridker PM, Lee IM. Physical activity and reduced risk of cardiovascular events: potential mediating mechanisms. Circulation. 2007;116(19):2110-2118.

31. Colberg SR, Sigal RJ, Fernhall B, et al. Exercise and type 2 diabetes: the American College of Sports Medicine and the American Diabetes Association: joint position statement. Diabetes Care. 2010;33(12):e147-167.

32. Yumuk V, Tsigos C, Fried M, et al. European Guidelines for Obesity Management in Adults. Obes Facts. 2015;8(6):402-424.

33. Anderson L, Taylor RS. Cardiac rehabilitation for people with heart disease: an overview of Cochrane systematic reviews. Cochrane Database Syst Rev. 2014(12):CD011273.

34. Taylor RS, Unal B, Critchley JA, Capewell S. Mortality reductions in patients receiving exercise-based cardiac rehabilitation: how much can be attributed to cardiovascular risk factor improvements? Eur J Cardiovasc Prev Rehabil. 2006;13(3):369-374.

35. Thomas DE, Elliott EJ, Naughton GA. Exercise for type 2 diabetes mellitus. Cochrane Database Syst Rev. 2006(3):CD002968.

36. Verheggen RJ, Maessen MF, Green DJ, Hermus AR, Hopman MT, Thijssen DH. A systematic review and meta-analysis on the effects of exercise training versus hypocaloric diet: distinct effects on body weight and visceral adipose tissue. Obes Rev. 2016;17(8):664-690.

37. Ross R, Dagnone $D$, Jones PJ, et al. Reduction in obesity and related comorbid conditions after diet-induced weight loss or exercise-induced weight loss in men. A randomized, controlled trial. Ann Intern Med. 2000;133(2):92-103.

38. Bandura A. Health promotion by social cognitive means. Health Educ Behav. 2004;31(2):143-164.

39. Miller, W., Rollnick, S. (2002). Motivational interviewing: Preparing people for change (2nd ed.). New York, NY: Guilford Press.

40. Hageman D, Fokkenrood HJ, Gommans $L N$, van den Houten MM, Teijink JA. Supervised exercise therapy versus home-based exercise therapy versus walking advice for intermittent claudication. Cochrane Database Syst Rev. 2018;4:CD005263.

41. Hageman D, van den Houten MM, Spruijt S, Gommans LN, Scheltinga MR, Teijink JA. Supervised exercise therapy: it does work, but how to set up a program? J Cardiovasc Surg (Torino). 2017;58(2):305-312.

42. Treat-Jacobson D, McDermott MM, Bronas UG, et al. Optimal Exercise Programs for Patients With Peripheral Artery Disease: A Scientific Statement From the American Heart Association. Circulation. 2019;139(4):e10-e33.

43. Lauret GJ, Fakhry F, Fokkenrood HJ, Hunink MG, Teijink JA, Spronk S. Modes of exercise training for intermittent claudication. Cochrane Database Syst Rev. 2014(7):CD009638.

44. Rozanski A, Bavishi C, Kubzansky LD, Cohen R. Association of Optimism With Cardiovascular Events and AllCause Mortality: A Systematic Review and Meta-analysis. JAMA Netw Open. 2019;2(9):e1912200.

45. Huffman JC, Beale EE, Celano CM, et al. Effects of Optimism and Gratitude on Physical Activity, Biomarkers, and Readmissions After an Acute Coronary Syndrome: The Gratitude Research in Acute Coronary Events Study. Circ Cardiovasc Qual Outcomes. 2016;9(1):55-63. 

Studies on the potential influence of supervised exercise therapy on cardiovascular risk, secondary interventions and mortality 



\section{Chapter}

\section{The Effects of Supervised Exercise Therapy on Modifiable Cardiovascular Risk Factors in Intermittent Claudication - A Systematic Review and}

Meta-Analysis

Jansen SCP

Hoorweg BBN

Hoeks SE

van den Houten MML

Scheltinga MRM

Teijink JAW

Rouwet EV

J Vasc Surg. 2019 Apr;69(4):1293-1308.e2 


\section{ABSTRACT}

\section{Objectives}

Cardiovascular events, such as myocardial infarction and stroke, contribute significantly to the prognosis of patients with peripheral artery disease. Therefore cardiovascular risk reduction is a vital element of treatment in patients with intermittent claudication (IC). The cardiovascular risk is largely determined by modifiable risk factors, which can be treated with medical care and lifestyle adjustments, such as increasing physical activity. The objective of this study was to determine the effects of supervised exercise therapy (SET) on modifiable cardiovascular risk factors in IC patients.

\section{Methods}

This is a systematic review and meta-analysis of prospective studies on the effects of SET on cardiovascular risk factors in symptomatic IC patients. Studies were eligible if they presented baseline and follow-up values for at least one of the following risk factors: blood pressure (systolic or diastolic), heart rate, lipid profile (total cholesterol, triglycerides, high-density lipoprotein cholesterol, lowdensity lipoprotein cholesterol), glucose, glycated haemoglobin, body weight, body mass index, or cigarette smoking. Pooled mean differences between follow-up and baseline were analysed using a random-effects model. Data were classified into short-term results (6 weeks-3 months) and midterm results (6-12 months). Statistical heterogeneity was presented as $\mathrm{I}^{2}$ and Q statistic.

\section{Results}

Twenty-seven studies with a total of 808 patients were included in this review. In the short term, SET resulted in significant improvements of systolic blood pressure (decrease of $4 \mathrm{~mm} \mathrm{Hg}$; 10 studies; 95\% confidence interval [CI], -6.40 to -1.76 ; I $^{2}$ 0\%) and diastolic blood pressure (decrease of $2 \mathrm{~mm} \mathrm{Hg}$; 8 studies; $95 \% \mathrm{Cl},-3.64$ to -0.22 ; $\left.I^{2} 35 \%\right)$. In the midterm, SET contributed to significant lowering of levels of lowdensity lipoprotein cholesterol (decrease of $0.2 \mathrm{mmol} / \mathrm{L}$; four studies; $95 \% \mathrm{Cl},-0.30$ to $-0.12 ; I^{2} 29 \%$ ) and total cholesterol (decrease of $0.2 \mathrm{mmol} / \mathrm{L}$, four studies; $95 \% \mathrm{Cl}$, -0.38 to $\left.-0.10 ; 1^{2} 36 \%\right)$. No significant effects of SET were identified for heart rate, triglycerides, high-density lipoprotein cholesterol, glucose, glycated haemoglobin, body weight, body mass index, or cigarette smoking.

\section{Conclusions}

This systematic review and meta-analysis shows favourable effects of SET on modifiable cardiovascular risk factors, specifically blood pressure and cholesterol levels. Despite the moderate quality, small trial sample sizes, and study heterogeneity, these findings support the prescription of SET programs not only to increase walking distances but also for risk factor modification. Future studies should address the potential effectiveness of SET to promote a healthier lifestyle and to improve cardiovascular outcomes in patients with claudication. 


\section{INTRODUCTION}

Peripheral artery disease (PAD) is an expression of systemic atherosclerosis and its prognosis is largely determined by cardiovascular events. The overall 5 -year mortality rate of PAD is approximately $20 \%$ with fatal cardiovascular events accounting for $70 \%$ of these deaths. An additional 30\% of PAD patients are confronted with a non-fatal cardiovascular event. ${ }^{1}$ Therefore, cardiovascular risk reduction is a vital element in the management of PAD patients. ${ }^{2,3}$ Important targets for reducing cardiovascular risk are the well-known modifiable risk factors including hypertension, dyslipidaemia, obesity, hyperglycaemia, tobacco smoking, and physical inactivity. ${ }^{4,5}$ These factors can be treated with medical treatment and lifestyle adjustments. ${ }^{6}$

Additionally, there might be a direct benefit following institution of supervised exercise therapy (SET). Exercise has been demonstrated to exert positive effects on cardiovascular risk factors and cardiovascular disease., ${ }^{7,8}$ Guidelines on secondary cardiovascular prevention acknowledge that many of the risk factor improvements occurring in cardiac rehabilitation can be mediated through exercise. ${ }^{9,10}$ As a consequence, exercise training is recommended as a central component of rehabilitation and preventive interventions. ${ }^{2,3,6,10,11}$

The most prevalent symptom of PAD is intermittent claudication (IC) defined as a cramping pain in calf, thigh or buttock muscles during walking that is relieved upon a short period of rest. SET is generally accepted as the optimal primary treatment to improve walking ability and quality of life in patients with IC. ${ }^{2,3}$ A typical SET program consists of intermittent bouts of walking to moderate-to-maximum claudication symptoms alternated with periods of rest in a recommended frequency of three 30-45 minute sessions per week for a minimum of 12 weeks. ${ }^{2}$ SET should be provided under the guidance of a qualified healthcare professional, such as a physical therapist, either in a hospital or a community-based setting. The majority of publications emphasize the (cost-)effectiveness of SET for improving walking distance and quality of life ${ }^{12,13}$, but relatively few studies focus on the effects of SET on cardiovascular risk factors in patients with IC. ${ }^{14-18}$

The aim of this review was to provide a systematic overview of the effectiveness of SET on modifying cardiovascular risk factors in patients with IC. This was assessed by (a) analysing pooled mean differences between baseline and follow-up (after SET) for common cardiovascular risk factors in IC patients, and (b) by conducting a meta-analysis of SET's effectiveness on reduction of cardiovascular risk factors versus usual care or invasive treatment. 


\section{METHODS}

This systematic review and meta-analysis was performed according to standards as described in the Preferred Reporting Items for Systematic reviews and MetaAnalysis (PRISMA) guideline. ${ }^{19}$ The protocol for this review has been published prior to data synthesis on the PROSPERO website (http://www.crd.york.ac.uk/PROSPERO) with registration number CRD42017080706.

\section{Eligibility criteria}

Randomized controlled trials, prospective cohort studies and case control studies were considered eligible for inclusion if they were conducted in patients with IC (symptomatic PAD Fontaine stage II or Rutherford 1-3) and if SET was tested as an intervention. Furthermore, a study was required to include both baseline and follow-up assessments of at least one of the following modifiable cardiovascular risk factors: blood pressure, heart rate, body weight, BMI, lipid profile, glycaemic status, and/or smoking status. SET was defined as treadmill or track based walking exercise performed under the supervision of medical professionals (e.g. physical therapists). SET was either hospital or community based with a minimum of one supervised session per week for at least six weeks. Nordic pole walking (NPW) was also considered as SET. Only original research published in English was included. Studies were excluded if the supervised exercise programme did not predominantly include walking exercise (e.g. circuit training, a programme with 'several sorts of exercise', 'dynamic leg exercises').

\section{Search strategy}

Several databases were searched with the assistance of a medical librarian (MEDLINE, EMBASE, Cochrane Central Register of Controlled Trials (CENTRAL), Web of Science, CINAHL EBSCOhost, and Google scholar) identifying articles from inception to April 2018. The following keywords were used: "claudication", "peripheral vascular disease", "peripheral occlusive artery disease", " angina cruris", "angiosclerotica intermittens", " leg ischemia", "kinesiotherapy", " exercise", "walking", "treadmill exercise", "aerobic exercise", "endurance training", "high intensity interval training", "leg exercise", "physiotherapy", "supervision", "physiotherapist", "rehabilitation'. See Addendum 1 for the complete search. Furthermore, reference lists of relevant articles were searched manually.

\section{Study selection}

Two authors (S.J. and B.H.) independently screened titles and abstracts and selected articles for inclusion through full-text evaluation. Disagreements between the two reviewers were resolved by discussion and consensus. 


\section{Outcomes}

This study assessed the change of a number of modifiable cardiovascular risk factors between baseline and follow-up. The outcomes of interest were resting blood pressure (systolic or diastolic), resting heart rate, body weight, body mass index, lipid profile (low-density lipoprotein (LDL), high-density lipoprotein (HDL), total cholesterol (TC), and triglycerides (TG)), glycaemic status ( $\mathrm{HbA} 1 \mathrm{C}$, and blood glucose), and smoking status.

\section{Data extraction}

A standardized data extraction form was used to obtain the following data of an included study: Characteristics of study and participants, description of SET intervention (content, frequency, duration, intensity, and amount of supervision), description of control group, duration of follow-up, and outcome measures at baseline and longest follow-up.

\section{Quality assessment}

Two investigators (S.J. and B.H.) independently assessed the quality of studies and any discrepancies were resolved by discussion. Multiple assessment tools were used because studies with different designs, including non-randomized studies, were also included in this review. Randomized controlled trials were assessed using the Cochrane Collaboration's Risk of Bias tool, categorizing studies into high, low and unclear risk. ${ }^{20}$ Case-control and cohort studies with control group were assessed using the ROBINS-I tool ${ }^{21}$, and the Quality Assessment Tool for Before-After Studies With No Control Group was used for before-after studies. ${ }^{22}$

\section{Statistical analysis}

Data were analysed using R Version 3.4.3. Analysis was executed and presented separately for each of the modifiable cardiovascular risk factors. Meta-analysis was performed if 3 or more studies were available per risk factor, otherwise results were presented narratively. Pooled mean differences between baseline and follow-up were analysed using a random-effects model according to DerSimonian and Laird ${ }^{23}$, to account for between-study variance. Data were divided into short-term follow-up (six weeks to three months) and mid-term follow-up (six to twelve months), because of expected variation in follow-up duration among studies. Change score data were converted into final values prior to entry into the pooled analysis. Where only change scores were provided, final value means were calculated using change score means and baseline data. Baseline intervention and control standard deviations were used to estimate final value standard deviations. Dichotomous outcomes were presented as relative risks. The heterogeneity was evaluated using the $1^{2}$ statistic, in which values from $25 \%, 50 \%$, and $75 \%$ correspond to low, moderate, and high heterogeneity, respectively, as well as the Q-statistic. ${ }^{24}$ Possible publication bias 
was expected to be high because of small sample sizes of studies. The number of studies did not allow for examining funnel plots for asymmetry.

To compare the effectiveness of SET on modifying risk factors to usual care or invasive revascularization, meta-analysis was conducted if 3 or more RCT's were available for a particular risk factor. Mean differences were calculated as a summary statistic using a random-effects model according to DerSimonian and Laird ${ }^{23}$, and were based on change-from-baseline scores and standard deviations. Where mean differences and standard deviations were not reported, standard deviations were calculated using a conservative correlation coefficient of 0.5 for within-patient correlation from baseline to follow-up.

\section{RESULTS}

\section{Literature search}

After removal of duplicates, 2999 potentially relevant records were identified (Figure 1). A total of 42 articles met our inclusion criteria. However, nine of these 42 articles did not qualify because of missing data that could not be collected through contact with the authors. Of the remaining articles, five appeared to be double publications necessitating omission from analysis. No additional articles were identified through the reference lists of relevant articles. Eventually, a total of 28 publications on 27 studies were included in this review.

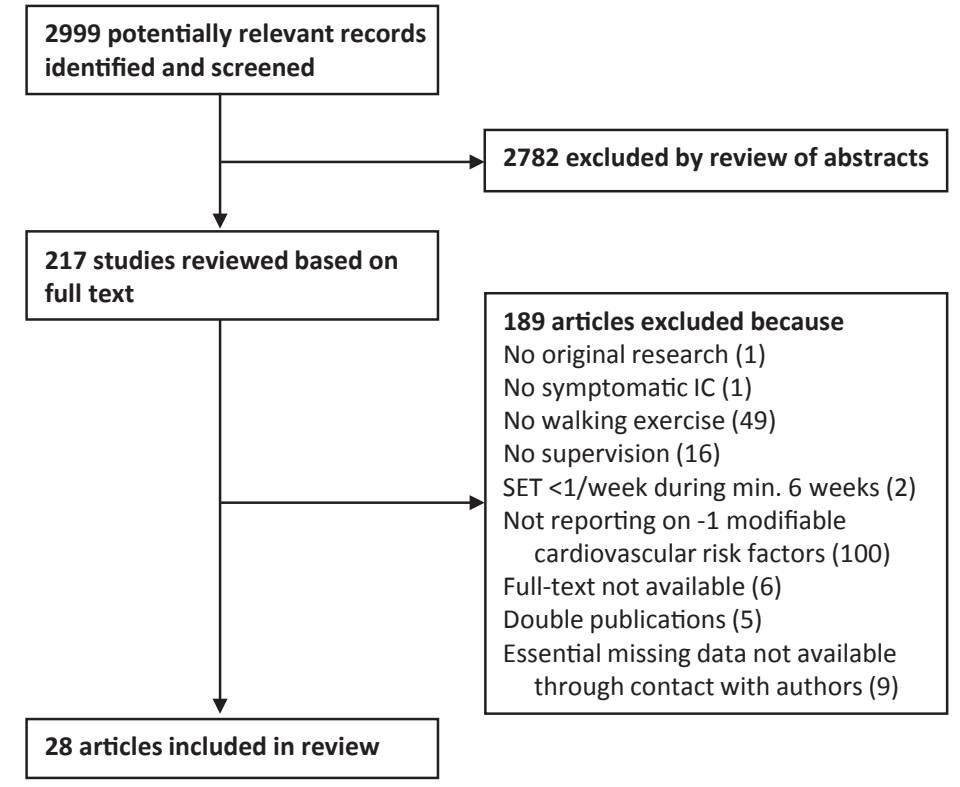

Figure 1 Flowchart of the study selection process. 


\section{Study characteristics}

Characteristics of the included studies are listed in Table I. This review included the results of 808 patients who received SET only (i.e. not in combination with invasive treatment). The mean age of the participants ranged from 57 to 74 years and the majority were male (42 to 100\%). The duration of the intervention and follow-up varied from 6 weeks to 12 months. A particularly short follow-up of 6 weeks was observed in three studies. ${ }^{25-27}$ The number of participants in the supervised walking groups varied from eight to 73 and ten studies reported follow-up data for less than twenty patients. Almost all studies described a reproducible method of SET, which mostly consisted of treadmill walking two to three times per week until the onset of pain or until moderate pain. Notably, one study ${ }^{27}$ stood out because of a relatively intensive SET program. For the study of McDermott et $\mathrm{al}^{28}$, which included patients with IC and asymptomatic PAD, the specific data of IC patients were collected.

Among the 26 included studies were fifteen RCT's. For the analysis of pooled mean differences between baseline and follow-up for the risk factors after SET only the risk factor data of the SET groups of these RCT'S were used. Two studies ${ }^{29,30}$ compared variations of SET programs, which were considered as two independent SET groups for this review.

\section{Study quality}

The quality assessment per study is summarized in the final column of Table I and the comprehensive assessment is presented in Addendum 2. Most studies were of fair or moderate methodological quality. All studies were considered at high risk of performance bias due to the nature of the intervention. Several studies did not report on blinding of outcome assessors and hampering whether knowledge of the intervention would substantially influence the outcomes of our interest. The risk of outcome reporting bias was generally low. 


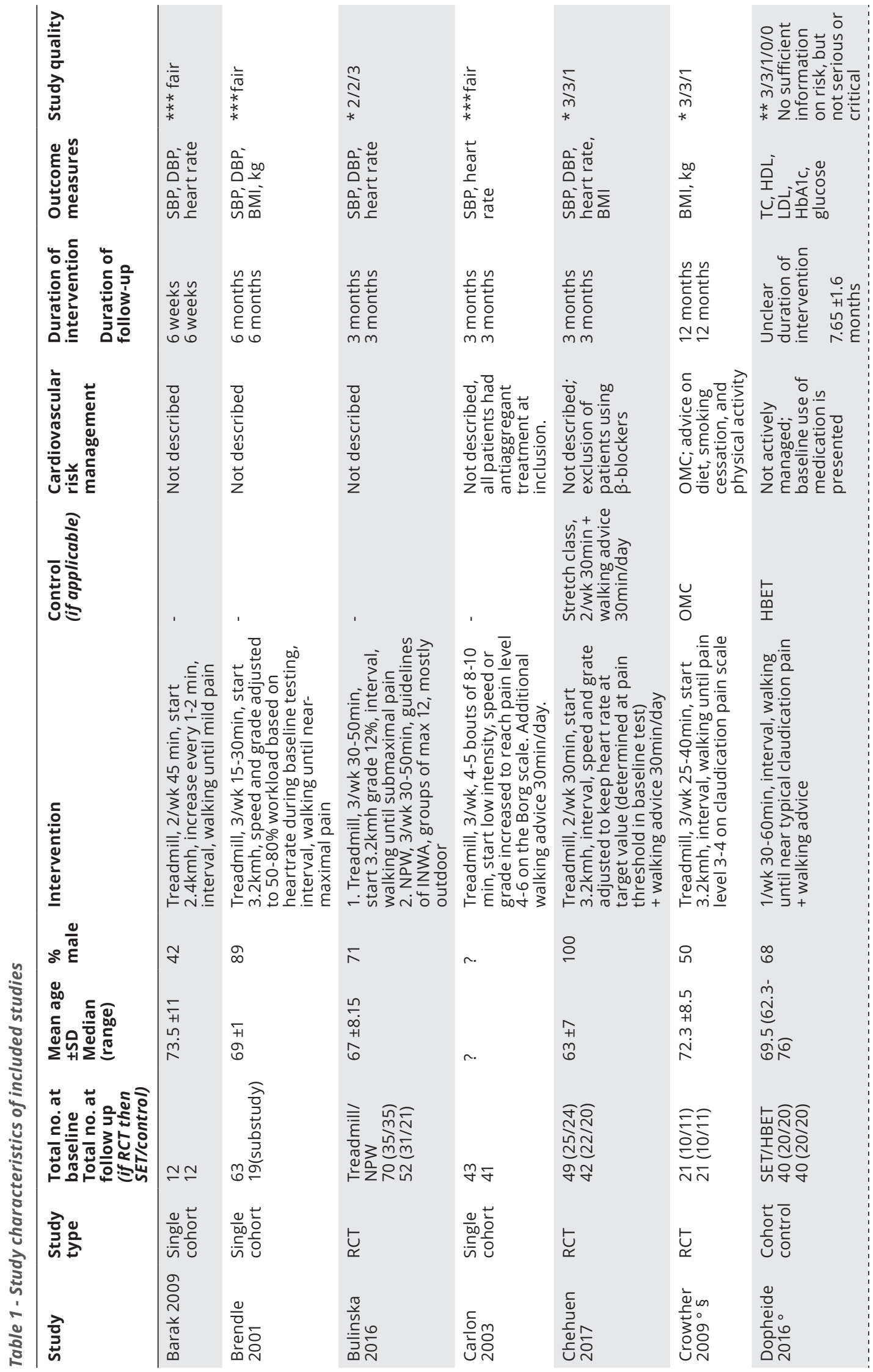




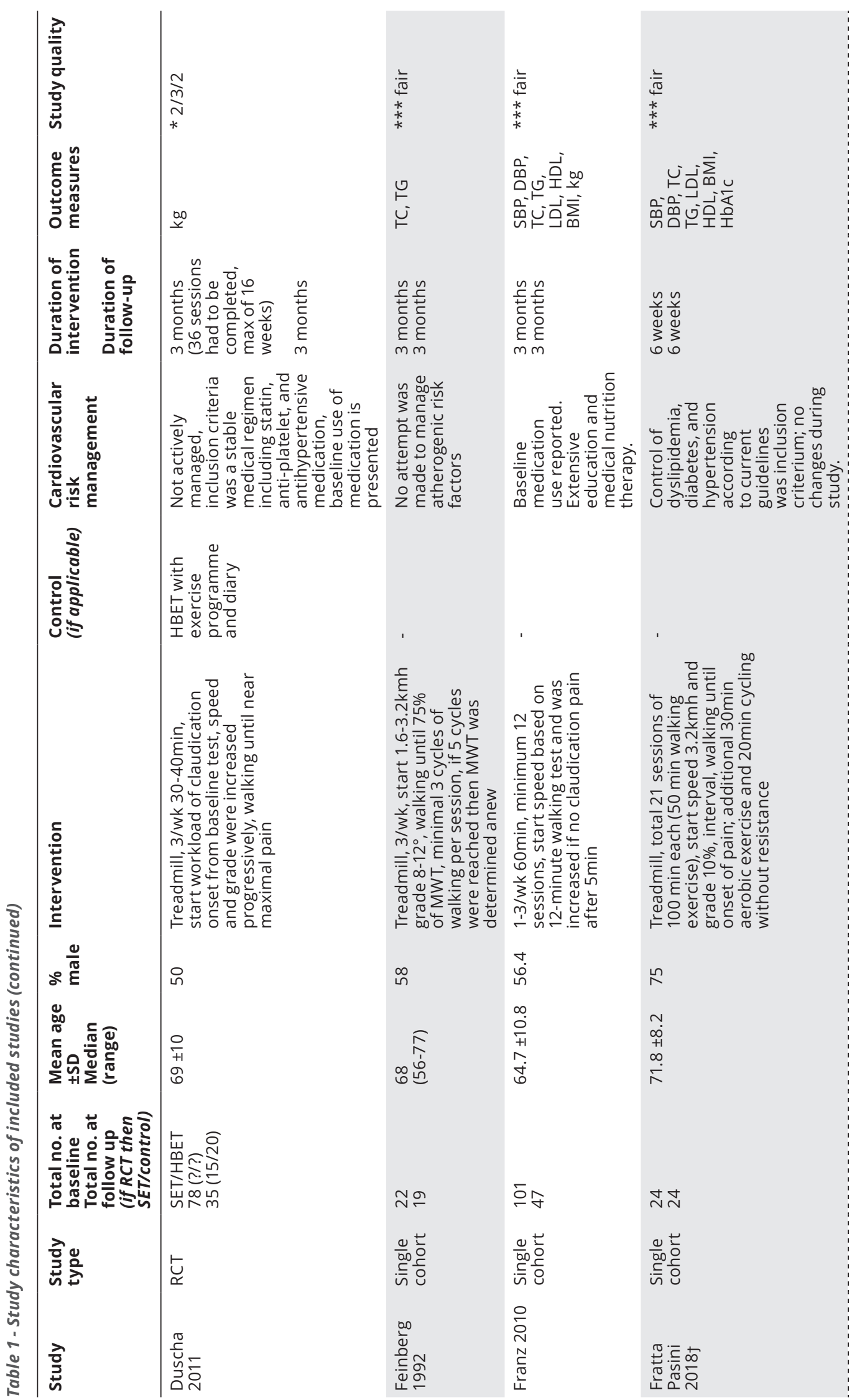




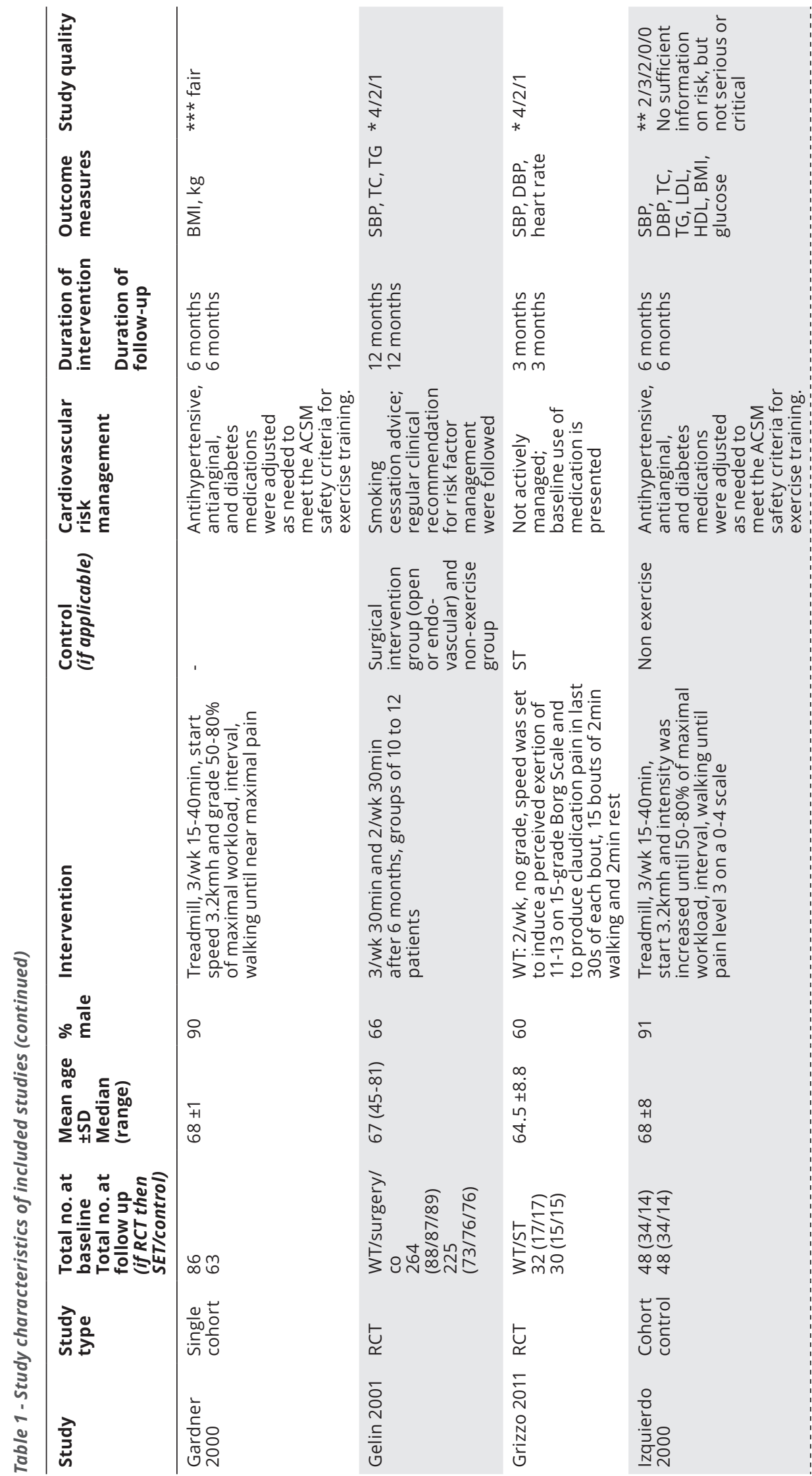




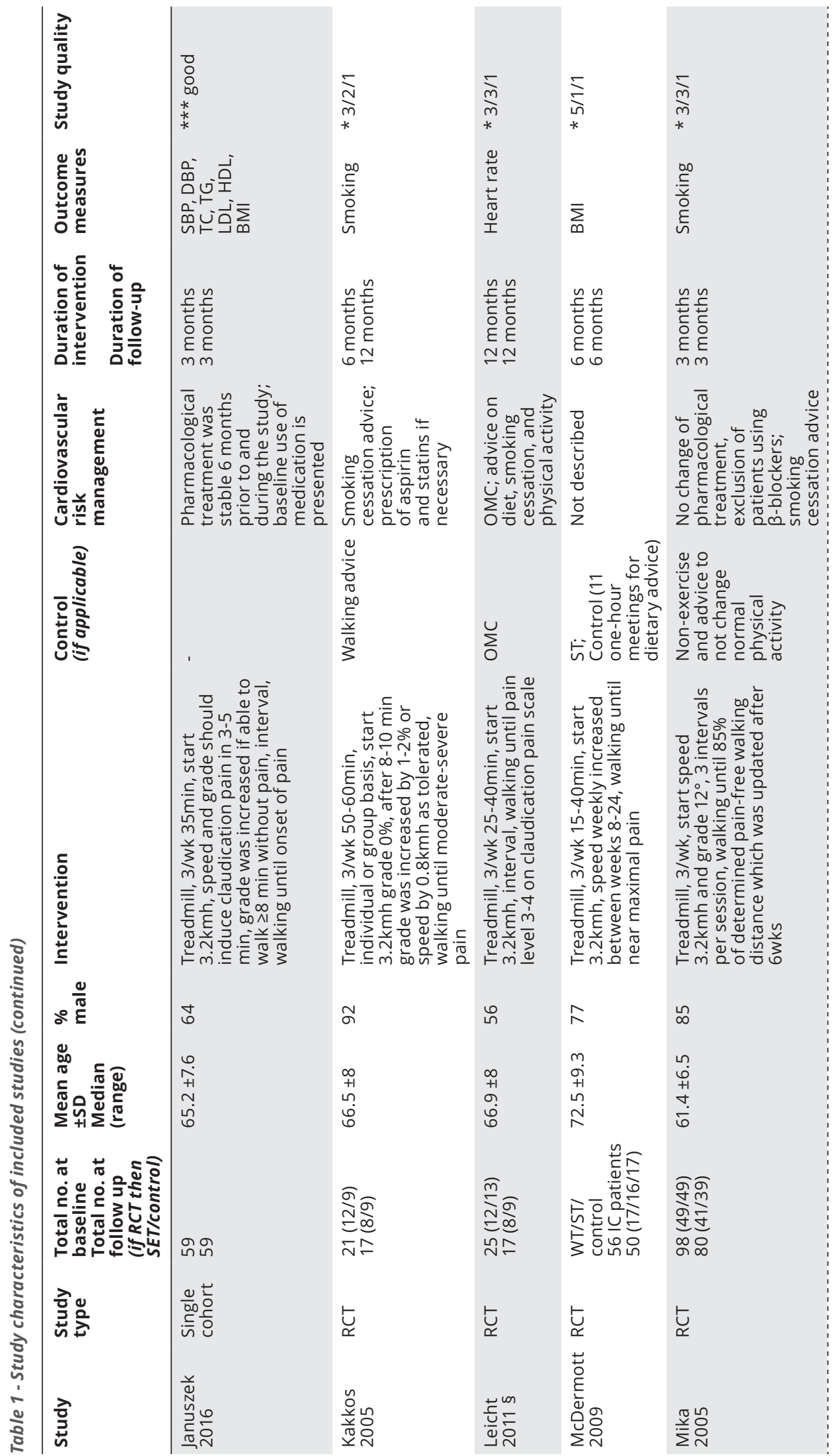




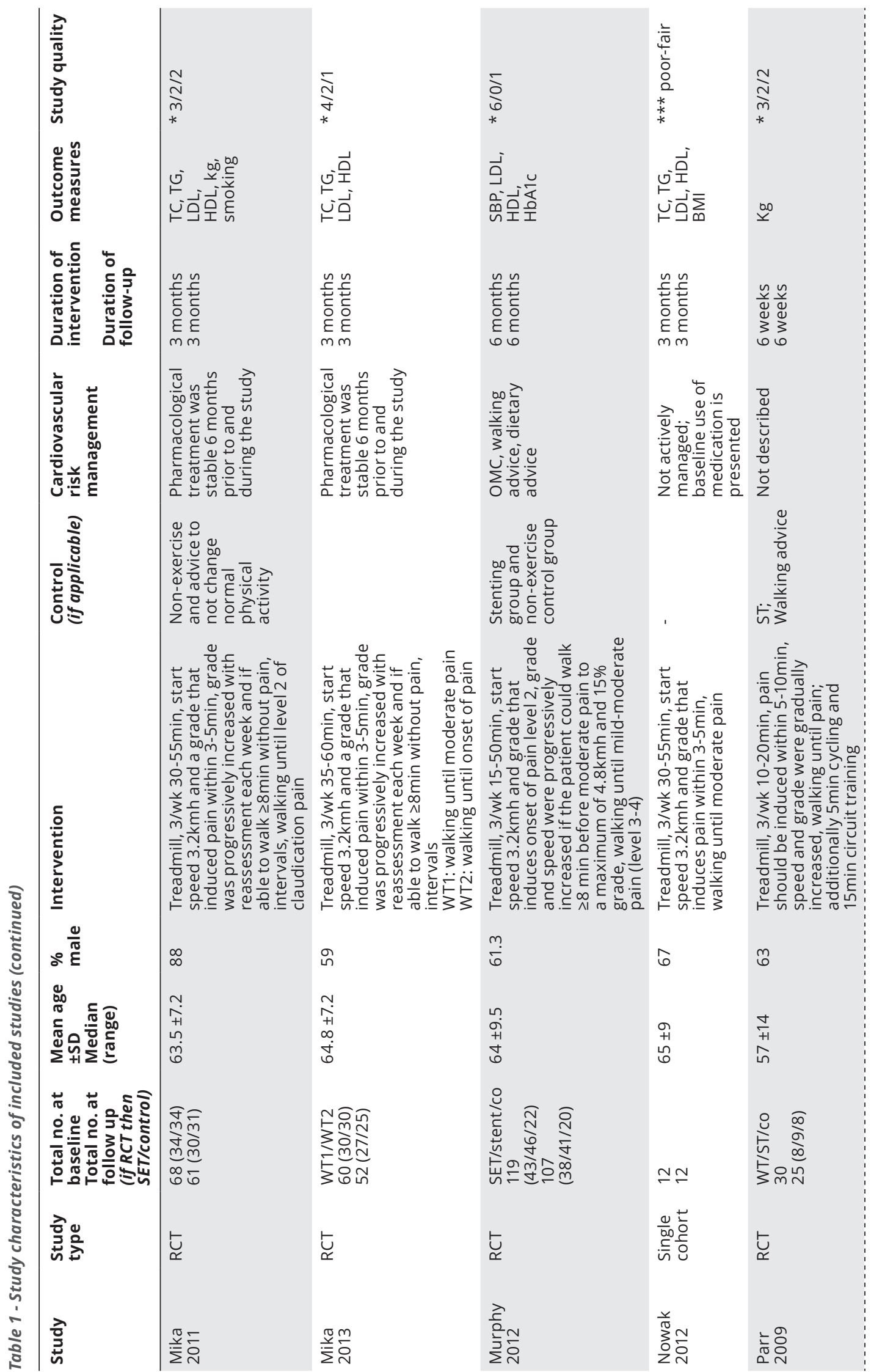




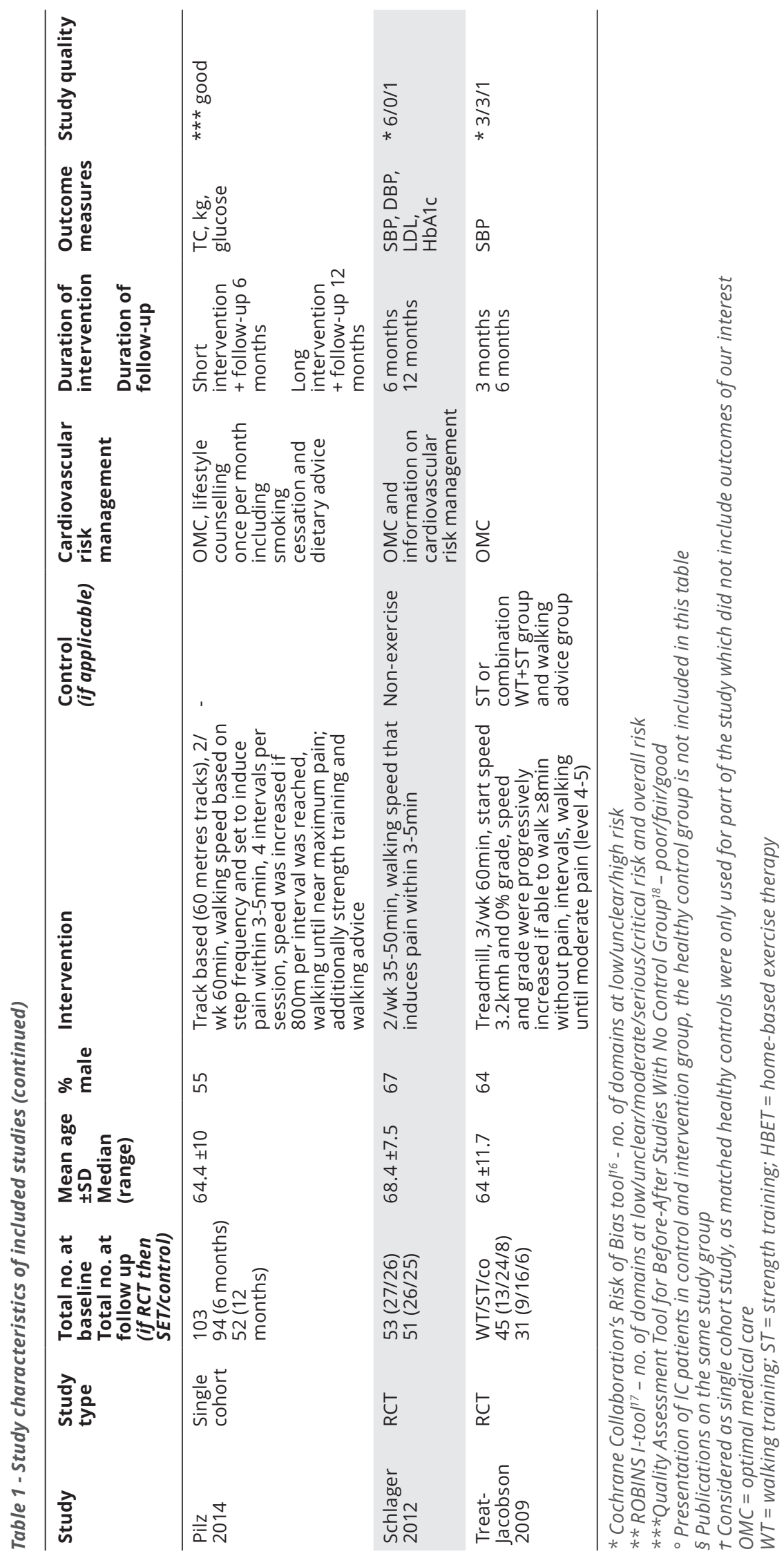




\section{Risk factor outcomes}

\section{Resting blood pressure}

Systolic blood pressure Sufficient data of fourteen studies were available (notably, the study of Bulinska et al ${ }^{29}$ was considered as two separate groups). Mean values at baseline ranged from 129 to $154 \mathrm{mmHg}$. A significant $4 \mathrm{mmHg}$ decrease was seen at short-term (10 studies, 95\% confidence interval [-6.40 to -1.76$], \mathrm{I}^{2} 0 \%$, Q 2.43, p 0.99), but not at mid-term follow-up (6 studies, $-1 \mathrm{mmHg}$ [-6.20 to 3.73], $I^{2}$ 86\%, Q 63.78, p 0.00) (Figure 2). Furthermore, meta-analysis of five RCT's which compared SET to usual care demonstrated a significant difference of $8 \mathrm{mmHg}$ ([-10.84 to -4.73], $\left.\right|^{2}$ 0\%) in favour of exercise on short-term follow-up, but no significant mid-term difference $\left(-3 \mathrm{mmHg},\left[-8.31\right.\right.$ to 2.03], $\left.\mathrm{I}^{2} 55 \%\right)$ (Figure 3). Furthermore, two RCT's were available which compared SET to invasive treatment, in which the latter existed of open or endovascular surgery in Gelin's study ${ }^{31}$ and of stenting in Murphy's study ${ }^{32}$. Neither found a significant intergroup difference.

Diastolic blood pressure The mean values at baseline ranged from 66 to 81 $\mathrm{mmHg}$ among included studies. A significant decrease of $2 \mathrm{mmHg}$ was seen at short-term (8 studies, [-3.64 to -0.22], I2 35\%, Q 10.85, p 0.21), but not at mid-term follow-up (3 studies, $-0.6 \mathrm{mmHg}$ [-2.40 to 1.23], I2 86\%, Q 17.25, p 0.00) (Supplemental figure 1). Two RCT's comparing SET to usual care ${ }^{14,33}$ found no significant change in either the exercise or the usual care groups.

Resting heart rate The mean values at baseline ranged from 68 to $74 \mathrm{bpm}$ among included studies. Analysis of the short-term outcomes of five studies presented a non-significant decrease of $1 \mathrm{bpm}$ ([-3.95 to 1.93], I2 0\%, Q 1.88, p 0.87) (Figure 4). Additionally, one study ${ }^{16}$ reported a decrease of 8 bpm (SD 14.8) in the SET group after 12 months of follow-up. Although this RCT found no decrease for the conservative control group (1.2 $\pm 11.3 \mathrm{bpm})$, the intergroup difference was not significant. Another $\mathrm{RCT}^{14}$ did show a significant intergroup difference ( $\left.\mathrm{p} 0.01\right)$ in heart rate change between walking exercise (significant decrease) and stretching control (no change).

\section{Body weight and Body Mass Index}

Body weight The mean values at baseline ranged from 79 to $89 \mathrm{~kg}$ in the analysed studies. There was no significant change at short-term (4 studies, $-1.6 \mathrm{~kg}$ [-5.00 to 1.83], I2 0\%, Q 0.38, p 0.94) or mid-term follow-up (4 studies, $-0.1 \mathrm{~kg}$ [-0.62 to 0.46], $I^{2} 0 \%, Q 0.08$, p 0.99) (Supplemental figure 2). Three RCT's ${ }^{18,25,34}$ compared SET to usual care, but the variation of follow-up duration ( 6 weeks to 12 months) did not allow for a meta-analysis. These RCT's found no significant change in body weight for the SET or usual care groups.

Body Mass Index The mean values at baseline ranged from 24.6 to $31 \mathrm{~kg} / \mathrm{m}^{2}$ among included studies. There was no significant change at short-term (5 studies, $-0.3 \mathrm{~kg} / \mathrm{m}^{2}$ [-1.13 to 0.57$], \mathrm{I}^{2} 0 \%$, 
Q 0.16, p 1.00) or mid-term follow-up (5 studies, $0.1 \mathrm{~kg} / \mathrm{m}^{2}$ [-0.09 to 0.25$], \mathrm{I}^{2}$ 0\%, $\mathrm{Q} 0.27, \mathrm{p}$ 0.99) (Figure 5). Three RCT's $\mathrm{s}^{14,28,34}$ compared SET to usual care and found no significant change for either group. Notably, these RCT's were not pooled for a metaanalysis because of the broad variation in follow-up duration (from 3 to 12 months).

\section{Lipid profile}

LDL-cholesterol The mean values at baseline ranged from 2.14 to $4.13 \mathrm{mmol} / \mathrm{L}$ in the analysed studies. A significant decrease of $0.2 \mathrm{mmol} / \mathrm{L}$ was seen at mid-term (4 studies, [-0.30 to -0.12], I2 29\%, Q 3.19, p 0.36), but not at short-term follow-up (7 studies, $-0.2 \mathrm{mmol} / \mathrm{L}$ [-0.41 to 0.10], I2 83\%, Q 39.22, p 0.00) (Figure 6). Three RCT's compared walking exercise to usual care, however meta-analysis was not possible because for one of these studies baseline and final values for the control group were not available. This particular study ${ }^{18}$ found no significant change in the control group compared to a significant decrease of LDL in the exercise group. Another study found no significant change in both groups. ${ }^{33}$ And the final study ${ }^{32}$ showed a decrease in LDL in the exercise group, conservative group, and invasive treatment group, however the intergroup differences were not significant (exercise vs. control $p=0.813$ and exercise vs. invasive $p=0.474$ ).

HDL-cholesterol The mean values at baseline ranged from 0.95 to $1.57 \mathrm{mmol} / \mathrm{L}$ among analysed studies. There was no significant change at short-term ( 6 studies, $0.1 \mathrm{mmol} / \mathrm{L}$ [0.00 to 0.10], $\mathrm{I}^{2}$ 0\%, Q 3.71, p 0.72) or mid-term follow-up (3 studies, $0.1 \mathrm{mmol} / \mathrm{L}$ [-0.02 to 0.19), I2 84\%, Q 15.87, p 0.00) (Supplemental figure 3). One $\mathrm{RCT}^{18}$ found a significant increase in HDL in the exercise group, but not in the control group. The CLEVER study ${ }^{32}$ found a significantly greater increase in HDL in the exercise group compared with the stenting group $(p=0.013)$, but no significant intergroup difference was found between exercise and conservative treatment $(p=0.551)$.

Total cholesterol The mean values at baseline ranged from 3.82 to $6.16 \mathrm{mmol} / \mathrm{L}$ among included studies. A significant decrease of $0.2 \mathrm{mmol} / \mathrm{L}$ was observed at midterm (4 studies, [-0.38 to -0.10], I2 36\%, Q 4.67, p 0.20), but not at short-term follow-up (7 studies, $-0.1 \mathrm{mmol} / \mathrm{L}$ [-0.40 to 0.20], I2 83\%, Q 47.74, p 0.00) (Supplemental figure 4). One RCT ${ }^{18}$ found a significant decrease in the exercise group, but not in the control group. In another $\mathrm{RCT}^{31}$, changes in total cholesterol in the supervised exercise group did not differ from changes in the surgical or conservatively treated groups.

Triglycerides The mean values at baseline ranged from 1.16 to $2.37 \mathrm{mmol} / \mathrm{L}$ among included studies. There was no significant change at short-term follow-up (7 studies, $-0.1 \mathrm{mmol} / \mathrm{L}$ [-0.14 to 0.01], I2 0\%, Q 9.16, p 0.24) (Supplemental figure 5). For mid-term follow-up only two studies ${ }^{15,31}$ were available, which did not allow for meta-analysis. Both studies reported no significant change. One of these studies ${ }^{31}$ also found no significant change after conservative or invasive treatment. Another $\mathrm{RCT}^{18}$ found a significant decrease in triglycerides after 3 months of SET, but not after conservative treatment. 


\section{Glycaemic profile}

HbA1c The mean values at baseline ranged from $6.08 \%$ to $6.10 \%$ in the analysed studies. The three available studies presented data on six months follow-up and meta-analysis showed no significant change (0.01\% [-0.09 to 0.10$]$, I $2 \%$, Q 0.05 , p 0.98) (Figure 7). One cohort study ${ }^{27}$ reported no significant change after 6 weeks of SET. One RCT33 found no significant change in the exercise or the conservative group. Another $\mathrm{RCT}^{32}$ found no significant change in the exercise, stenting or conservative group and also no significant intergroup differences.

Blood glucose The mean values at baseline ranged from 5.9 to $6.8 \mathrm{mmol} / \mathrm{L}$ among included studies. The three available studies presented data on six months followup and meta-analysis showed no significant change $(-0.1 \mathrm{mmol} / \mathrm{L}$ [- 0.24 to 0.02$]$, $I^{2} 0 \%, Q$ 0.80, p 0.67) (Supplemental figure 6).

smoking Only three studies reported on changes in smoking status. One study ${ }^{35}$ described that the two patients which were current smokers at baseline (41 pack years) reduced smoking by at least $40 \%$ at follow-up. Two RCT's ${ }^{18,36}$ comparing SET to usual care reported on the reduction of the amount of cigarettes smoked per day, but found no significant intergroup differences. 


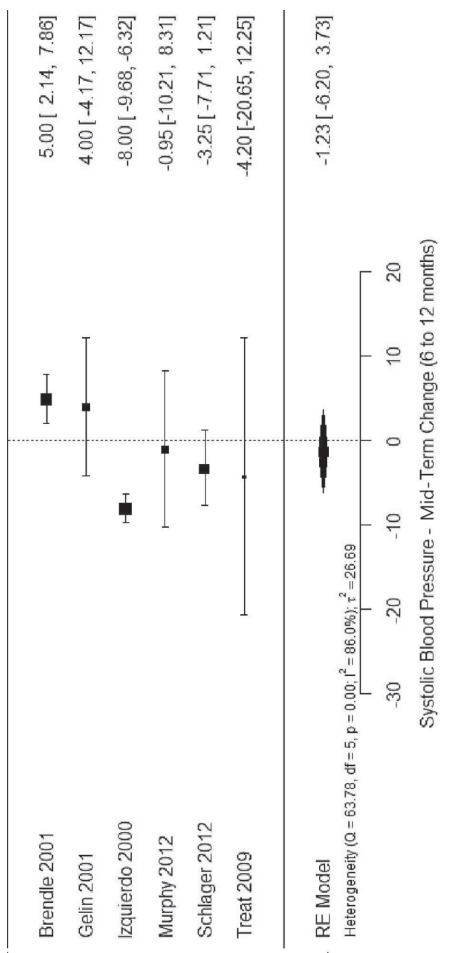

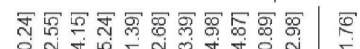

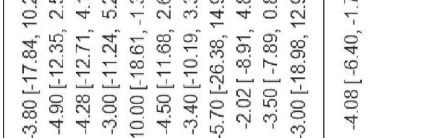



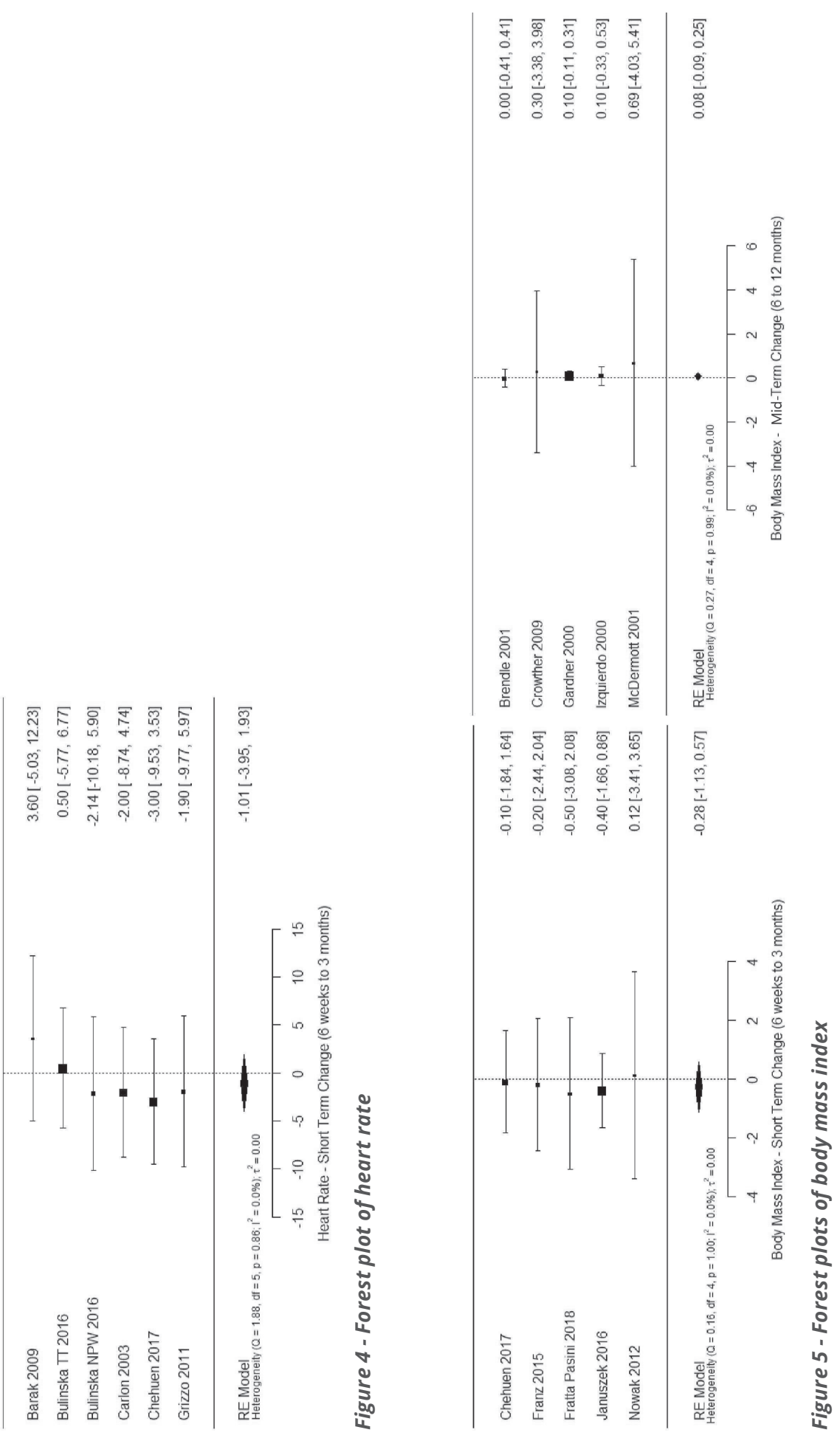

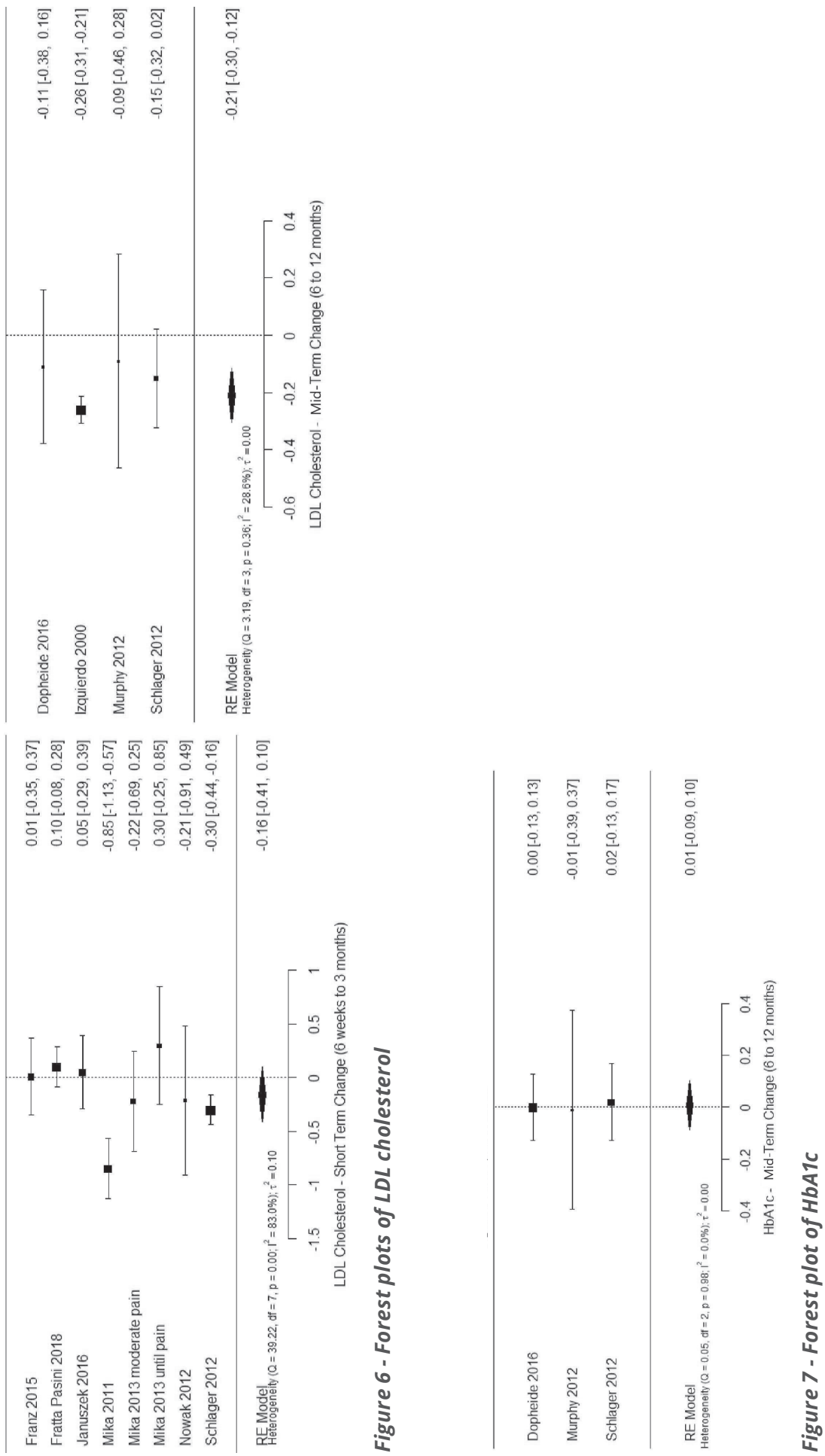


\section{DISCUSSION}

This review provides an overview of the effects of SET on modifiable cardiovascular risk factors in patients with IC. Our findings show that SET contributes to a reduction of systolic and diastolic blood pressure in the short term, and a lowering of LDLcholesterol and total cholesterol in the mid-term. However, these findings should be interpreted cautiously as included studies were of small sample size, moderate study quality, heterogeneous, and the outcomes of interest were mostly reported as secondary outcomes.

Exercise is an important element of cardiovascular risk management. Previous studies in other patient populations report on ample positive effects of exercise on cardiovascular risk factors.,8 For example, exercise has been shown to lower blood pressure, irrespective of baseline blood pressure levels $6,11,37-41$, and reduces LDL-cholesterol and non-HDL cholesterol levels. ${ }^{11,37,39,40,42}$ The results of the current systematic review are in line with these findings. Although the reductions found seem limited, especially the effect on systolic blood pressure is clinically relevant as this reduction of $4 \mathrm{mmHg}$ reduces cardiovascular risk and all-cause mortality by $8 \%$ and $5 \%$, respectively. ${ }^{43}$

However, it is unclear to what extent the observed effects are actually related to the exercise intervention. Surely, exercise is recommended as a central component of various rehabilitation programs as there is compelling evidence that exercise is an essential means of improving cardiovascular risk factors. ${ }^{2,3,6,10,11}$ Nevertheless, it is likely that the observed reductions in blood pressure and cholesterol levels are at least in part due to medication, as treatment of hypertension and dyslipidaemia should be an integral part of optimal medical care (OMC) in all patients with PAD. Since medication use was incompletely reported in most of included studies, it was not possible to distinguish between the overall effects of SET and OMC. A meta-analysis comparing SET to usual care was only possible for systolic blood pressure at short-term follow-up and showed that SET was superior to usual care in reducing systolic blood pressure. In this analysis, the prevalence of hypertension was similar among the control and SET groups. ${ }^{14,33,44}$ In two of these studies all participants received OMC. The third study ${ }^{14}$ did not explicitly mention OMC as part of treatment and only documented baseline use of antihypertensive agents which was 55\% among controls and $73 \%$ in the SET group. Regarding lipid control, the RCT of Mika et al ${ }^{18}$ presented a large decline in serum lipid levels in favour of the SET group, while medication was kept stable 6 months prior to and during the study period, suggesting a direct effect of exercise training. Although we could not establish the effect ratios of exercise versus pharmacotherapy, it is evident that both therapies should be an integral part of cardiovascular risk management.

SET did not have a significant effect on glycaemic profile and body weight in this comprehensive review. Based on available literature, such effects were not necessarily expected in this review's population. Regarding the glycaemic profile, 
most studies with improved glycaemic control after exercise are conducted in participants with impaired glucose tolerance or type 2 diabetes and investigate the effect of extended programs (i.e. several years). ${ }^{38}$ Optimal results are obtained in programs combining aerobic and resistance training with intensive lifestyle interventions (including dietary interventions). ${ }^{7,38,45}$ Similarly, in overweight or obese patients, the effect of exercise alone on weight loss is modest, though it aids in long-term weight maintenance. ${ }^{37,46}$ Furthermore, exercise reduces visceral adiposity which is a stronger predictor for morbidity and mortality than body weight per se. ${ }^{47-49}$ In this review, the lack of effect on glycaemic profile and body weight may be expected as baseline values of these parameters were mostly within the normal range, although one study ${ }^{50}$ reported a $43 \%$ prevalence of obesity. Moreover, SET in IC patients usually consists solitary of aerobic exercise and most of the programs did not contain intensive lifestyle management. It may therefore be argued that a combination of SET and intensive lifestyle management will probably optimize results.

One may assume that any SET-associated reduction in cardiovascular risk favourably influences the prognosis of PAD patients. Several reports highlight the importance of exercise in improving cardiovascular outcome. Exercise-based rehabilitation in cardiac patients diminishes the number of hospitalisations and reduces cardiovascular mortality up to $30 \% .6,39,40,51,52 \mathrm{~A}$ recent systematic review in PAD patients reported a 0.71 risk ratio for mortality in favour of exercise compared to control. ${ }^{53}$ Furthermore, a prospective study even reported a $52 \%$ reduction of cardiovascular mortality and 30\% reduction of morbidity after 12 weeks of SET.54 As data on clinical outcomes such as cardiac morbidity and mortality were not regularly reported in most SET studies, the current review focused on cardiovascular risk factors as intermediate outcomes. Importantly, these risk factors have proven to be predictive of cardiovascular risk and are therefore clinically relevant outcomes. ${ }^{40}$ However, given the lack of data on the effects of SET on cardiovascular risk factors, it is not possible to draw firm conclusions on the potential benefits of SET in terms of cardiovascular risk management. Future studies with long-term follow-up should address the potential effectiveness of SET to improve overall cardiovascular health in PAD patients.

\section{Limitations and weaknesses}

This meta-analysis has limitations that should be addressed. First, only articles written in English were included. Second, many studies had non-randomized designs and the methodological quality of studies was moderate at the most. In particular, unclear or high ratings for risk of bias were found for blinding. Study quality could be underestimated, as blinding of participants and personnel was not possible due to the nature of the intervention and the outcomes of interest were objective measurements (e.g. laboratory tests), implying a low impact of bias. A number of studies were of small sample size and the outcomes of interest for this review were 
mostly presented as secondary outcomes, increasing the risk of publication bias. For some risk factors, the number of studies was not sufficient to execute a metaanalysis to determine the effects of SET, while a meta-analysis for the comparison of SET to usual care or invasive treatment was only possible for systolic blood pressure. Finally, heterogeneity across included studies was substantial due to differences in types of study design, exact content of SET, and duration of follow-up. To handle this variation, a random-effects model was used in the meta-analysis and data were divided into subgroups of short-term and mid-term follow-up. Furthermore, the $I^{2}$ and $\mathrm{Q}$ values were presented for transparency.

\section{Future research recommendations}

Although the available evidence might not be sufficient to draw firm conclusions about the explicit effects of SET on cardiovascular risk in IC patients, it is not desirable to perform future studies to compare the effectiveness of SET to usual care. That is, SET combined with OMC is an established recommendation for the primary treatment of $\mathrm{IC}$, precluding randomization into control groups in future studies on ethical grounds. Moreover, there is solid evidence indicating beneficial effects of regular exercise in both healthy and chronically ill patients. Therefore, future treatment and research should focus on expanding the intensity of lifestyle management and counselling as an addition to SET. This approach would most likely create more durable effects of SET and is supposed to have a synergetic effect on the modifiable risk factors. To effectuate this, implementation barriers to SET should be overcome, especially accessibility, referral, and adherence, and physiotherapists should be educated in competencies to motivate, educate, and support patients in changing their lifestyle.

\section{CONCLUSION}

This review shows a significant reduction of blood pressure and cholesterol levels after SET in IC patients. These findings support the standard prescription of SET programs not only to increase walking distances in patients with claudication, but also for risk factor modification. The next step is to expand on intensive lifestyle management and counselling as an integrative part of current SET programs. Future studies should address the potential effectiveness of such integrated programs to promote a healthy lifestyle and improve cardiovascular outcomes in patients with claudication.

\section{ACKNOWLEDGEMENTS}

The authors would like to express their great appreciation to WM Bramer (Medical Library, Erasmus MC) for his assistance in developing the search strategies. 


\section{REFERENCES}

1. Lauret GJ, Van Dalen DCW, Willigendael EM, et al. Supervised exercise therapy for intermittent claudication: Current status and future perspectives. Vascular. 2012;20(1):12-19.

2. Gerhard-Herman MD, Gornik HL, Barrett C, et al. 2016 AHA/ACC Guideline on the Management of Patients With Lower Extremity Peripheral Artery Disease: Executive Summary: A Report of the American College of Cardiology/American Heart Association Task Force on Clinical Practice Guidelines. Circulation. 2017;135(12):e686-e725.

3. Aboyans V, Ricco JB, Bartelink MEL, et al. ESC Guidelines on the Diagnosis and Treatment of Peripheral Arterial Diseases, in collaboration with the European Society for Vascular Surgery (ESVS). Eur J Vasc Endovasc Surg. 2017; 10.1016/j.ejvs.2017.07.018.

4. Yu E, Rimm E, Qi L, et al. Diet, Lifestyle, Biomarkers, Genetic Factors, and Risk of Cardiovascular Disease in the Nurses' Health Studies. Am J Public Health. 2016;106(9):1616-1623.

5. Berger JS, Hochman J, Lobach I, Adelman MA, Riles TS, Rockman CB. Modifiable risk factor burden and the prevalence of peripheral artery disease in different vascular territories. J Vasc Surg. 2013;58(3):673-681 e671.

6. Perk J, De Backer G, Gohlke H, et al. European Guidelines on cardiovascular disease prevention in clinical practice (version 2012). The Fifth Joint Task Force of the European Society of Cardiology and Other Societies on Cardiovascular Disease Prevention in Clinical Practice (constituted by representatives of nine societies and by invited experts). Eur Heart J. 2012;33(13):1635-1701.
7. Pedersen BK, Saltin B. Exercise as medicine - evidence for prescribing exercise as therapy in 26 different chronic diseases. Scand J Med Sci Sports. 2015;25 Suppl 3:1-72.

8. Kujala UM. Evidence on the effects of exercise therapy in the treatment of chronic disease. Br J Sports Med. 2009;43(8):550-555.

9. European Association of Cardiovascular P, Rehabilitation Committee for Science G, Eacpr, et al. Secondary prevention through cardiac rehabilitation: physical activity counselling and exercise training: key components of the position paper from the Cardiac Rehabilitation Section of the European Association of Cardiovascular Prevention and Rehabilitation. Eur Heart J. 2010;31(16):1967-1974.

10. Smith SC, Jr., Benjamin EJ, Bonow RO, et al. AHA/ACCF secondary prevention and risk reduction therapy for patients with coronary and other atherosclerotic vascular disease: 2011 update: a guideline from the American Heart Association and American College of Cardiology Foundation endorsed by the World Heart Federation and the Preventive Cardiovascular Nurses Association. J Am Coll Cardiol. 2011;58(23):2432-2446.

11. Eckel RH, Jakicic JM, Ard JD, et al. 2013 AHA/ACC guideline on lifestyle management to reduce cardiovascular risk: a report of the American College of Cardiology/American Heart Association Task Force on Practice Guidelines. J Am Coll Cardiol. 2014;63(25 Pt B):2960-2984.

12. Hageman D, Fokkenrood HJ, Gommans LN, van den Houten MM, Teijink JA. Supervised exercise therapy versus home-based exercise therapy versus walking advice for intermittent claudication. Cochrane Database Syst Rev. 2018;4:CD005263. 
13. Fakhry F, Fokkenrood HJ, Spronk S, Teijink JA, Rouwet EV, Hunink MGM. Endovascular revascularisation versus conservative management for intermittent claudication. Cochrane Database Syst Rev. 2018;3:CD010512.

14. Chehuen M, Cucato GG, Carvalho CRF, et al. Walking training at the heart rate of pain threshold improves cardiovascular function and autonomic regulation in intermittent claudication: A randomized controlled trial. J Sci Med Sport. 2017;20(10):886-892.

15. Izquierdo-Porrera AM, Gardner AW, Powell CC, Katzel LI. Effects of exercise rehabilitation on cardiovascular risk factors in older patients with peripheral arterial occlusive disease. J Vasc Surg. 2000;31(4):670-677.

16. Leicht AS, Crowther RG, Golledge J. Influence of peripheral arterial disease and supervised walking on heart rate variability. J Vasc Surg. 2011;54(5):13521359.

17. Grizzo Cucato G, de Moraes Forjaz CL, Kanegusuku $\mathrm{H}$, et al. Effects of walking and strength training on resting and exercise cardiovascular responses in patients with intermittent claudication. Vasa J Vasc Dis. 2011;40(5):390-397.

18. Mika P, Wilk B, Mika A, Marchewka A, Nizankowski R. The effect of painfree treadmill training on fibrinogen, haematocrit, and lipid profile in patients with claudication. Eur J Cardiovasc Prev Rehabil. 2011;18(5):754-760.

19. Moher D, Liberati A, Tetzlaff J, Altman DG, Group P. Preferred reporting items for systematic reviews and metaanalyses: the PRISMA statement. BMJ. 2009;339:b2535.

20. Higgins JP, Altman DG, Gotzsche PC, et al. The Cochrane Collaboration's tool for assessing risk of bias in randomised trials. BMJ. 2011;343:d5928.
21. Sterne JA, Hernan MA, Reeves BC, et al. ROBINS-I: a tool for assessing risk of bias in non-randomised studies of interventions. BMJ. 2016;355:i4919.

22. HealthNIo. Quality Assessment Tool for Before-After (Pre-Post) Studies with no Control Group. 2014; https://www. nhlbi.nih.gov/health-pro/guidelines/indevelop/cardiovascular-risk-reduction/ tools/before-after. Accessed January, 2018.

23. DerSimonian R, Laird N. Meta-analysis in clinical trials. Control Clin Trials. 1986;7(3):177-188.

24. Higgins JP, Thompson SG, Deeks JJ, Altman DG. Measuring inconsistency in meta-analyses. BMJ. 2003;327(7414):557560.

25. Parr BM, Noakes TD, Derman EW. Peripheral arterial disease and intermittent claudication: Efficacy of short-term upper body strength training, dynamic exercise training, and advice to exercise at home. S Afr Med J. 2009;99(11):800-804.

26. Barak S, Stopka CB, Archer Martinez C, Carmeli E. Benefits of low-intensity painfree treadmill exercise on functional capacity of individuals presenting with intermittent claudication due to peripheral arterial disease. Angiology. 2009;60(4):477-486.

27. Fratta Pasini AM, Stranieri C, Rigoni $A M$, et al. Physical Exercise Reduces Cytotoxicity and Up-Regulates Nrf2 and UPR Expression in Circulating Cells of Peripheral Artery Disease Patients: An Hypoxic Adaptation? 2018.

28. McDermott MM, Ades P, Guralnik JM, et al. Treadmill exercise and resistance training in patients with peripheral arterial disease with and without intermittent claudication: A randomized controlled trial. J Am Med Assoc. 2009;301(2):165174. 
29. Bulińska K, Kropielnicka K, Jasiński T, et al. Nordic pole walking improves walking capacity in patients with intermittent claudication: a randomized controlled trial. Disabil Rehabil. 2016;38(13):13181324.

30. Mika P, Konik A, Januszek R, et al. Comparison of two treadmill training programs on walking ability and endothelial function in intermittent claudication. Int J Cardiol. 2013;168(2):838-842.

31. Taft C, Karlsson J, Gelin J, et al. Treatment efficacy of intermittent claudication by surgical intervention, supervised physical exercise training compared to no treatment in unselected randomised patients I: One year results of functional and physiological improvements. Eur J Vasc Endovasc Surg. 2001;22(2):107-113.

32. Murphy TP, Cutlip DE, Regensteiner JG, et al. Supervised exercise versus primary stenting for claudication resulting from aortoiliac peripheral artery disease: six-month outcomes from the claudication: exercise versus endoluminal revascularization (CLEVER) study. Circulation. 2012;125(1):130-139.

33. Schlager O, Hammer A, Giurgea A, et al. Impact of exercise training on inflammation and platelet activation in patients with intermittent claudication. Swiss Med Wkly. 2012;142.

34. Crowther RG, Spinks WL, Leicht AS, Sangla K, Quigley F, Golledge J. The influence of a long term exercise program on lower limb movement variability and walking performance in patients with peripheral arterial disease. Hum Mov Sci. 2009;28(4):494-503.

35. Kakkos SK, Geroulakos G, Nicolaides AN. Improvement of the walking ability in intermittent claudication due to superficial femoral artery occlusion with supervised exercise and pneumatic foot and calf compression: A randomised controlled trial. Eur J Vasc Endovasc Surg. 2005;30(2):164-175.
36. Mika P, Spodaryk K, Cencora A. Effects of treadmill training on walking distance and lower limb blood flow in patients with intermittent claudication. Rehabilitacja Medyczna. 2005;9(1):3-9.

37. Yumuk V, Tsigos C, Fried M, et al. European Guidelines for Obesity Management in Adults. Obes Facts. 2015;8(6):402-424.

38. Colberg SR, Sigal RJ, Yardley JE, et al. Physical Activity/Exercise and Diabetes: A Position Statement of the American Diabetes Association. Diabetes Care. 2016;39(11):2065-2079.

39. Anderson L, Taylor RS. Cardiac rehabilitation for people with heart disease: an overview of Cochrane systematic reviews. Cochrane Database Syst Rev. 2014(12):CD011273.

40. Taylor RS, Unal B, Critchley JA, Capewell S. Mortality reductions in patients receiving exercise-based cardiac rehabilitation: how much can be attributed to cardiovascular risk factor improvements? Eur J Cardiovasc Prev Rehabil. 2006;13(3):369-374.

41. Douketis JD, Macie C, Thabane L, Williamson DF. Systematic review of longterm weight loss studies in obese adults: clinical significance and applicability to clinical practice. Int J Obes (Lond). 2005;29(10):1153-1167.

42. Colberg SR, Sigal RJ, Fernhall B, et al. Exercise and type 2 diabetes: the American College of Sports Medicine and the American Diabetes Association: joint position statement. Diabetes Care. 2010;33(12):e147-167.

43. Ettehad D, Emdin CA, Kiran A, et al. Blood pressure lowering for prevention of cardiovascular disease and death: a systematic review and meta-analysis. Lancet. 2016;387(10022):957-967. 
44. Treat-Jacobson D, Bronas UG, Leon AS. Efficacy of arm-ergometry versus treadmill exercise training to improve walking distance in patients with claudication. Vasc Med. 2009;14(3):203213.

45. Orozco LJ, Buchleitner AM, GimenezPerez G, Roque IFM, Richter B, Mauricio D. Exercise or exercise and diet for preventing type 2 diabetes mellitus. Cochrane Database Syst Rev. 2008(3):CD003054.

46. Mathus-Vliegen L, Toouli J, Fried M, et al. World Gastroenterology Organisation global guidelines on obesity. J Clin Gastroenterol. 2012;46(7):555-561.

47. Thomas DE, Elliott EJ, Naughton GA. Exercise for type 2 diabetes mellitus. Cochrane Database Syst Rev. 2006(3):CD002968.

48. Verheggen RJ, Maessen MF, Green DJ, Hermus AR, Hopman MT, Thijssen DH. A systematic review and meta-analysis on the effects of exercise training versus hypocaloric diet: distinct effects on body weight and visceral adipose tissue. Obes Rev. 2016;17(8):664-690.

49. Ross R, Dagnone D, Jones PJ, et al. Reduction in obesity and related comorbid conditions after diet-induced weight loss or exercise-induced weight loss in men. A randomized, controlled trial. Ann Intern Med. 2000;133(2):92-103.

50. Franz RW, Garwick T, Haldeman K. Initial results of a 12-week, institution-based, supervised exercise rehabilitation program for the management of peripheral arterial disease. Vascular. 2010;18(6):325-335.

51. Anderson L, Thompson DR, Oldridge N, et al. Exercise-based cardiac rehabilitation for coronary heart disease. Cochrane Database Syst Rev. 2016(1):CD001800.
52. Piepoli MF, Davos C, Francis DP, Coats AJ, ExTra MC. Exercise training metaanalysis of trials in patients with chronic heart failure (ExTraMATCH). BMJ. 2004;328(7433):189.

53. Lane R, Harwood A, Watson L, Leng GC. Exercise for intermittent claudication Review. Cochrane Database Syst Rev. 2017;12:CD000990.

54. Sakamoto S, Yokoyama N, Tamori Y, Akutsu K, Hashimoto H, Takeshita S. Patients with peripheral artery disease who complete 12-week supervised exercise training program show reduced cardiovascular mortality and morbidity. Circ J. 2009;73(1):167-173. 


\section{ADDENDUM 1 - COMPLETE SEARCH}

\section{Embase.com 2288}

('kinesiotherapy'/exp OR 'exercise'/de OR 'walking'/de OR 'treadmill exercise'/de OR treadmill/de OR 'aerobic exercise'/de OR 'endurance training'/de OR 'exercise intensity'/de OR ‘high intensity interval training'/de OR 'leg exercise'/de OR ‘muscle exercise'/de OR 'resistance training'/de OR physiotherapy/de OR (kinesiotherap* OR kinesitherap* OR exercis* OR walking OR ((treadmill* OR tread-mill*) NOT ((treadmill* OR tread-mill*) NEXT/1 test*)) OR ((training OR physical*) NEAR/3 therap*) OR (physical* NEAR/3 train*) OR physiokinesitherap* OR physiotherap*):ab,ti) AND (claudication/exp OR 'peripheral vascular disease'/de OR 'peripheral occlusive artery disease'/de OR (claudica* OR (peripher* NEAR/3 (vascul* OR vessel* OR arter*) NEAR/3 (disease* OR occlus* OR rehab* OR pathol*)) OR (arteriosclero* NEAR/3 obliter*) OR (peripher* NEAR/3 arteriopath*)):ab,ti) AND ('kinesiotherapy'/exp OR 'supervision'/de OR 'physiotherapist'/de OR physiotherapy/de OR 'rehabilitation'/ de OR rehabilitation:Ink OR ‘nurse'/de OR ‘treadmill exercise'/de OR treadmill/de OR (supervis* OR physiotherap* OR rehabilit* OR nurse* OR kinesiotherap* OR kinesitherap* OR ((exercis* OR physical*) NEAR/6 (therap* OR treat* OR train* OR program*)) OR ((treadmill* OR tread-mill*) NOT ((treadmill* OR tread-mill*) NEXT/1 test*))):ab,ti) NOT ([animals]/lim NOT [humans]/lim) NOT ([Conference Abstract]/lim OR [Letter]/lim OR [Note]/lim OR [Editorial]/lim) AND [english]/lim

\section{Medline Ovid 1730}

(exp Exercise therapy/ OR Exercise/ OR Walking/ OR Exercise Movement Techniques/ OR High-Intensity Interval Training/ OR Resistance Training/ OR Physical Therapy Modalities/ OR (kinesiotherap* OR kinesitherap* OR exercis* OR walking OR ((treadmill* OR tread-mill*) NOT ((treadmill* OR tread-mill*) ADJ test*)) OR ((training OR physical*) ADJ3 therap*) OR (physical* ADJ3 train*) OR physiokinesitherap* OR physiotherap*).ab,ti.) AND (Intermittent Claudication/ OR Peripheral Vascular Diseases/ OR (claudica* OR (peripher* ADJ3 (vascul* OR vessel* OR arter*) ADJ3 (disease* OR occlus* OR rehab* OR pathol*)) OR (arteriosclero* ADJ3 obliter*) OR (peripher* ADJ3 arteriopath*)).ab,ti.) AND (exp Exercise therapy/ OR Physical Therapists/ OR Physical Therapy Modalities/ OR rehabilitation/ OR rehabilitation. fs. OR nurses/ OR (supervis* OR physiotherap* OR rehabilit* OR nurse* OR kinesiotherap* OR kinesitherap* OR ((exercis* OR physical*) ADJ6 (therap* OR treat* OR train* OR program*)) OR ((treadmill* OR tread-mill*) NOT ((treadmill* OR treadmill*) ADJ test*))).ab,ti.) NOT (exp animals/ NOT humans/) NOT (letter OR news OR comment OR editorial OR congresses OR abstracts).pt. AND english.la.

\section{CINAHL EBSCOhost 418}

(MH Therapeutic Exercise + OR MH Exercise OR MH Walking OR MH Resistance Training OR MH Physical Therapy OR TI (kinesiotherap* OR kinesitherap* OR 
exercis* OR walking OR ((treadmill* OR tread-mill*) NOT ((treadmill* OR treadmill*) N1 test*)) OR ((training OR physical*) N2 therap*) OR (physical* N2 train*) OR physiokinesitherap* OR physiotherap*) OR AB (kinesiotherap* OR kinesitherap* OR exercis* OR walking OR ((treadmill* OR tread-mill*) NOT ((treadmill* OR treadmill*) N1 test*)) OR ((training OR physical*) N2 therap*) OR (physical* N2 train*) OR physiokinesitherap* OR physiotherap*)) AND (MH Intermittent Claudication OR MH Peripheral Vascular Diseases OR TI (claudica* OR (peripher* N2 (vascul* OR vessel* OR arter*) N2 (disease* OR occlus* OR rehab* OR pathol*)) OR (arteriosclero* N2 obliter*) OR (peripher* N2 arteriopath*)) OR AB (claudica* OR (peripher* N2 (vascul* OR vessel* OR arter*) N2 (disease* OR occlus* OR rehab* OR pathol*)) OR (arteriosclero* N2 obliter*) OR (peripher* N2 arteriopath*))) AND (MH Therapeutic Exercise + OR MH Physical Therapists OR MH Physical Therapy OR MH rehabilitation OR MH nurses OR TI (supervis* OR physiotherap* OR rehabilit* OR nurse* OR kinesiotherap* OR kinesitherap* OR ((exercis* OR physical*) N5 (therap* OR treat* OR train* OR program*)) OR ((treadmill* OR tread-mill*) NOT ((treadmill* OR treadmill*) N1 test*))) OR AB (supervis* OR physiotherap* OR rehabilit* OR nurse* OR kinesiotherap* OR kinesitherap* OR ((exercis* OR physical*) N5 (therap* OR treat* OR train* OR program*)) OR ((treadmill* OR tread-mill*) NOT ((treadmill* OR treadmill*) N1 test*))) NOT (MH animals+ NOT humans+) NOT PT (letter OR news OR comment OR editorial OR congresses OR abstracts) AND LA (english)

\section{Cochrane CENTRAL $\mathbf{5 2 3}$}

((kinesiotherap* OR kinesitherap* OR exercis* OR walking OR ((treadmill* OR tread-mill*) NOT ((treadmill* OR tread-mill*) NEXT/1 test*)) OR ((training OR physical*) NEAR/3 therap*) OR (physical* NEAR/3 train*) OR physiokinesitherap* OR physiotherap*):ab,ti) AND ((claudica* OR (peripher* NEAR/3 (vascul* OR vessel* OR arter*) NEAR/3 (disease* OR occlus* OR rehab* OR pathol*)) OR (arteriosclero* NEAR/3 obliter*) OR (peripher* NEAR/3 arteriopath*)):ab,ti) AND ((supervis* OR physiotherap* OR rehabilit* OR nurse* OR kinesiotherap* OR kinesitherap* OR ((exercis* OR physical*) NEAR/6 (therap* OR treat* OR train* OR program*)) OR ((treadmill* OR tread-mill*) NOT ((treadmill* OR tread-mill*) NEXT/1 test*))):ab,ti)

\section{Web of science 1064}

$\mathrm{TS}=((($ kinesiotherap* OR kinesitherap* OR exercis* OR walking OR ((treadmill* OR tread-mill*) NOT ((treadmill* OR tread-mill*) NEAR/1 test*)) OR ((training OR physical*) NEAR/2 therap*) OR (physical* NEAR/2 train*) OR physiokinesitherap* OR physiotherap*)) AND ((claudica* OR (peripher* NEAR/2 (vascul* OR vessel* OR arter*) NEAR/2 (disease* OR occlus* OR rehab* OR pathol*)) OR (arteriosclero* NEAR/2 obliter*) OR (peripher* NEAR/2 arteriopath*))) AND ((supervis* OR physiotherap* OR rehabilit* OR nurse* OR kinesiotherap* OR kinesitherap* OR ((exercis* OR physical*) NEAR/5 (therap* OR treat* OR train* OR program*)) OR 
((treadmill* OR tread-mill*) NOT ((treadmill* OR tread-mill*) NEAR/1 test*)))) ) AND DT=(article) AND LA=(english)

\section{Google scholar 200}

kinesiotherapy|exercise|walking|physiotherapy claudication|"peripher vascul|vessel|artery disease|occlus" supervised|supervision

\section{ADDENDUM 2 - QUALITY ASSESSMENT SUMMARY OF INCLUDED STUDIES ACCORDING TO THE USED ASSESSMENT TOOL}

\begin{tabular}{|c|c|c|c|c|c|c|c|}
\hline $\begin{array}{l}\text { Cochrane } \\
\text { Collaboration's } \\
\text { Risk of Bias tool }\end{array}$ & 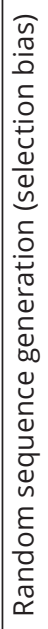 & 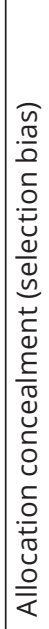 & 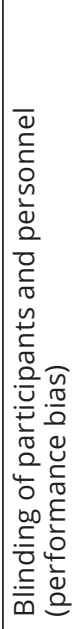 & 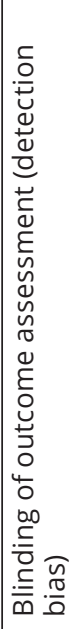 & 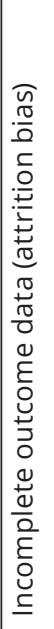 & 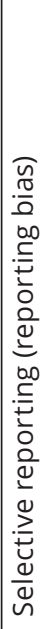 & $\begin{array}{l}\frac{n}{0} \\
\frac{0}{0} \\
\frac{1}{d} \\
\frac{1}{+} \\
0\end{array}$ \\
\hline \multicolumn{8}{|l|}{ Bulinska 2016} \\
\hline \multicolumn{8}{|l|}{ Chehuen 2017} \\
\hline \multicolumn{8}{|l|}{ Crowther 2009* } \\
\hline \multicolumn{8}{|l|}{ Duscha 2011} \\
\hline \multicolumn{8}{|l|}{ Gelin 2001} \\
\hline \multicolumn{8}{|l|}{ Grizzo 2011} \\
\hline \multicolumn{8}{|l|}{ Kakkos 2005} \\
\hline \multicolumn{8}{|l|}{ McDermott 2009} \\
\hline \multicolumn{8}{|l|}{ Mika 2005} \\
\hline \multicolumn{8}{|l|}{ Mika 2011} \\
\hline \multicolumn{8}{|l|}{ Mika 2013} \\
\hline \multicolumn{8}{|l|}{ Murphy 2012} \\
\hline \multicolumn{8}{|l|}{ Parr 2009} \\
\hline \multicolumn{8}{|l|}{ Schlager 2012} \\
\hline Treat-Jacobson 2009 & & & & & & & \\
\hline
\end{tabular}



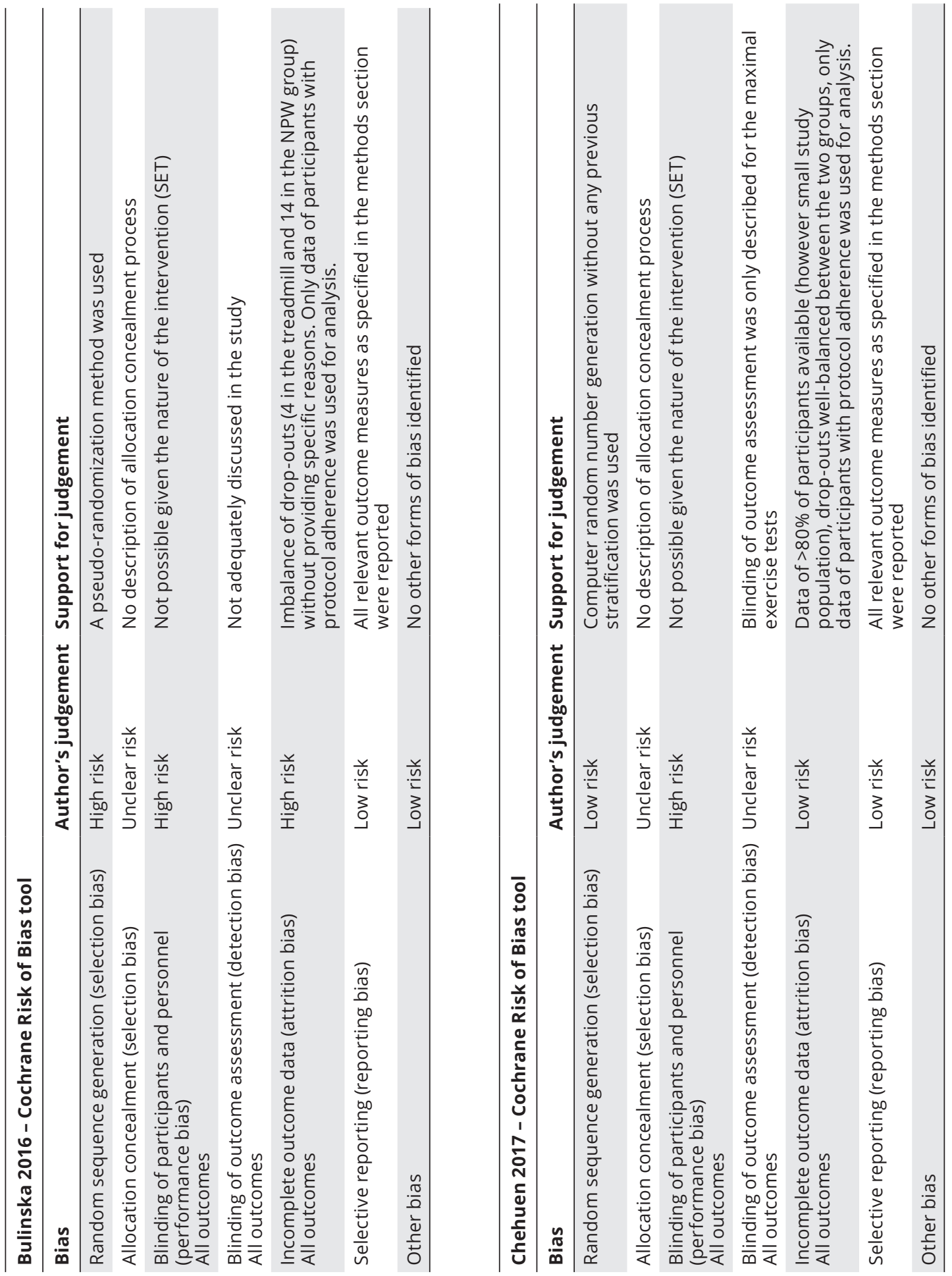

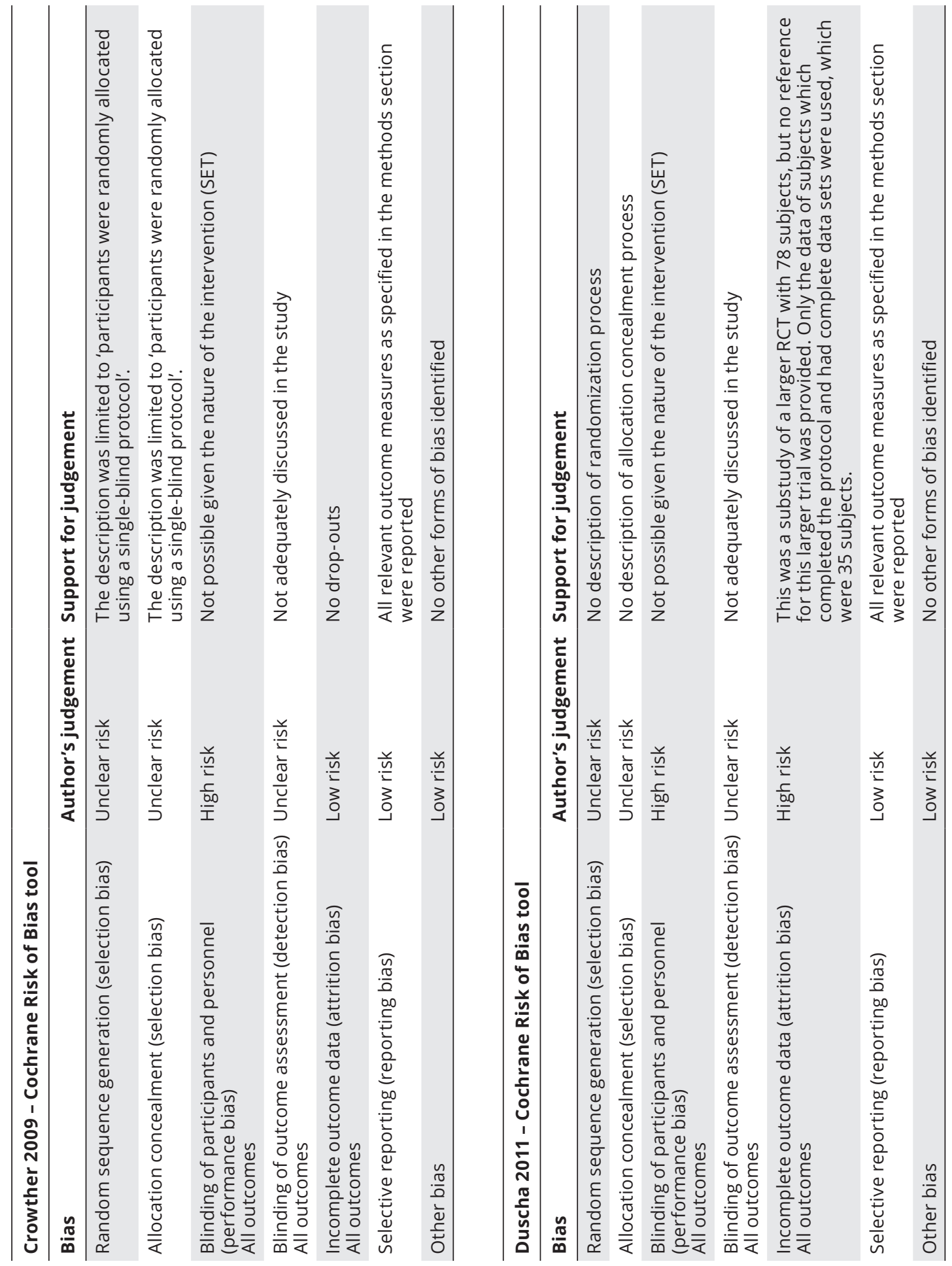


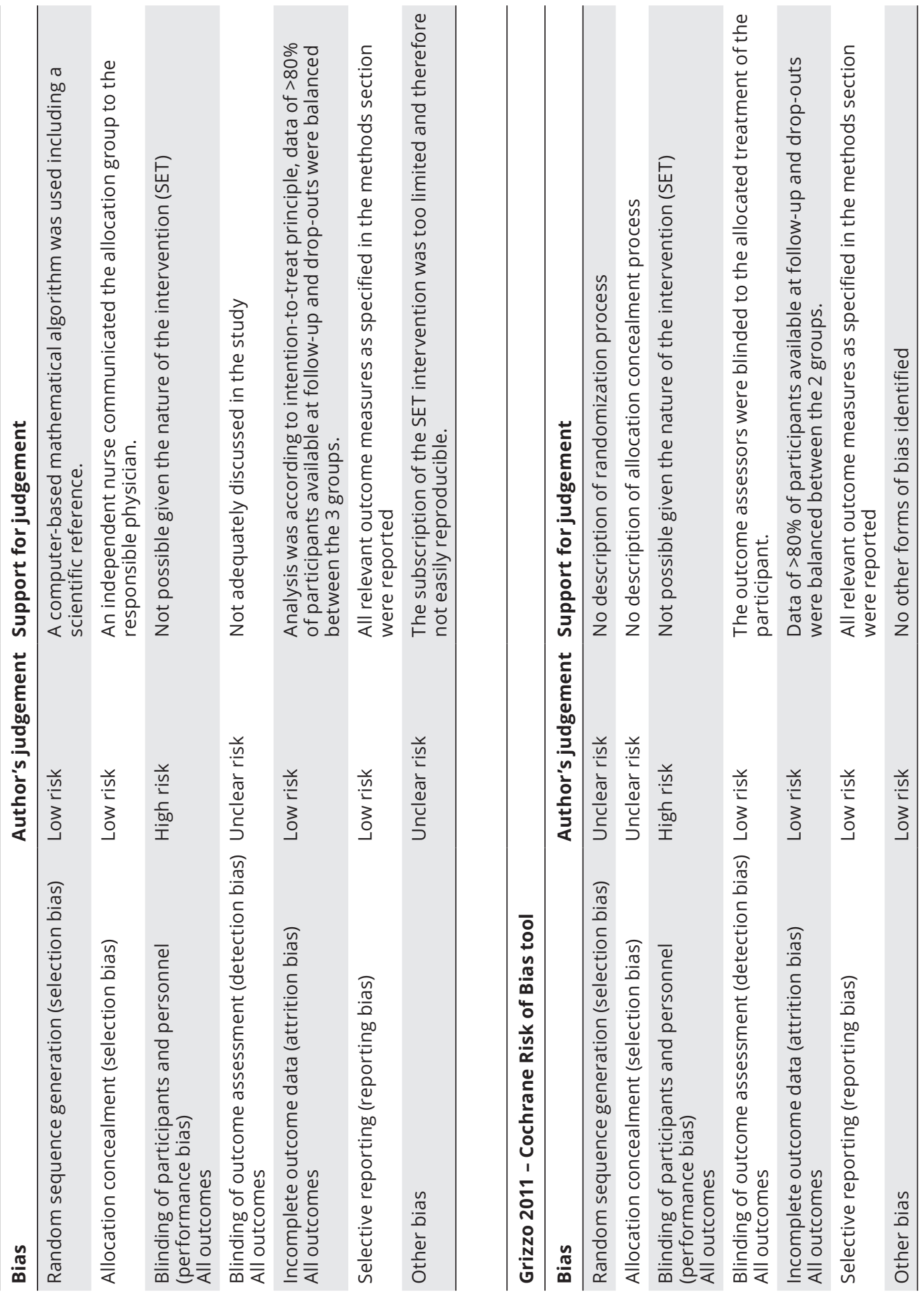



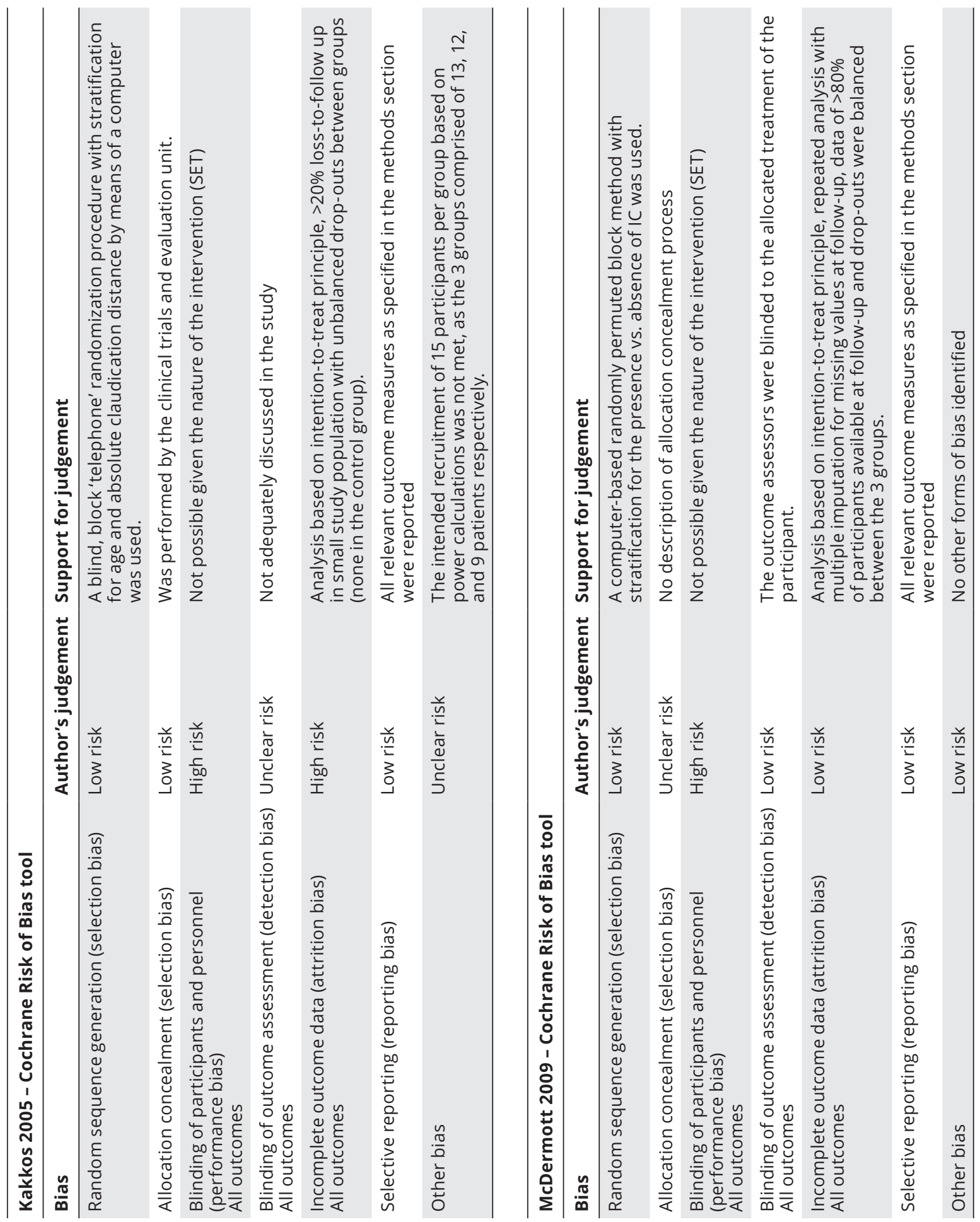

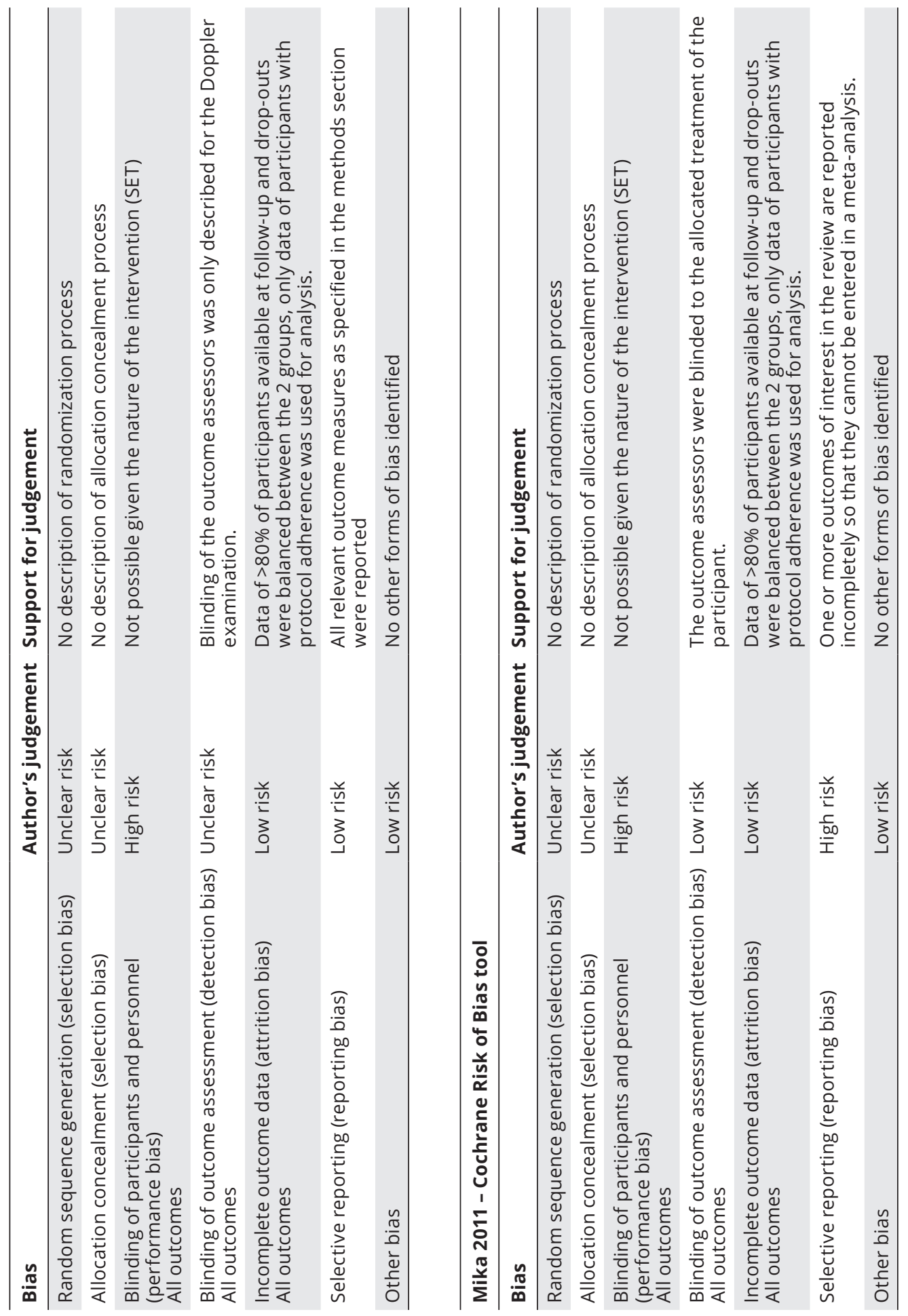

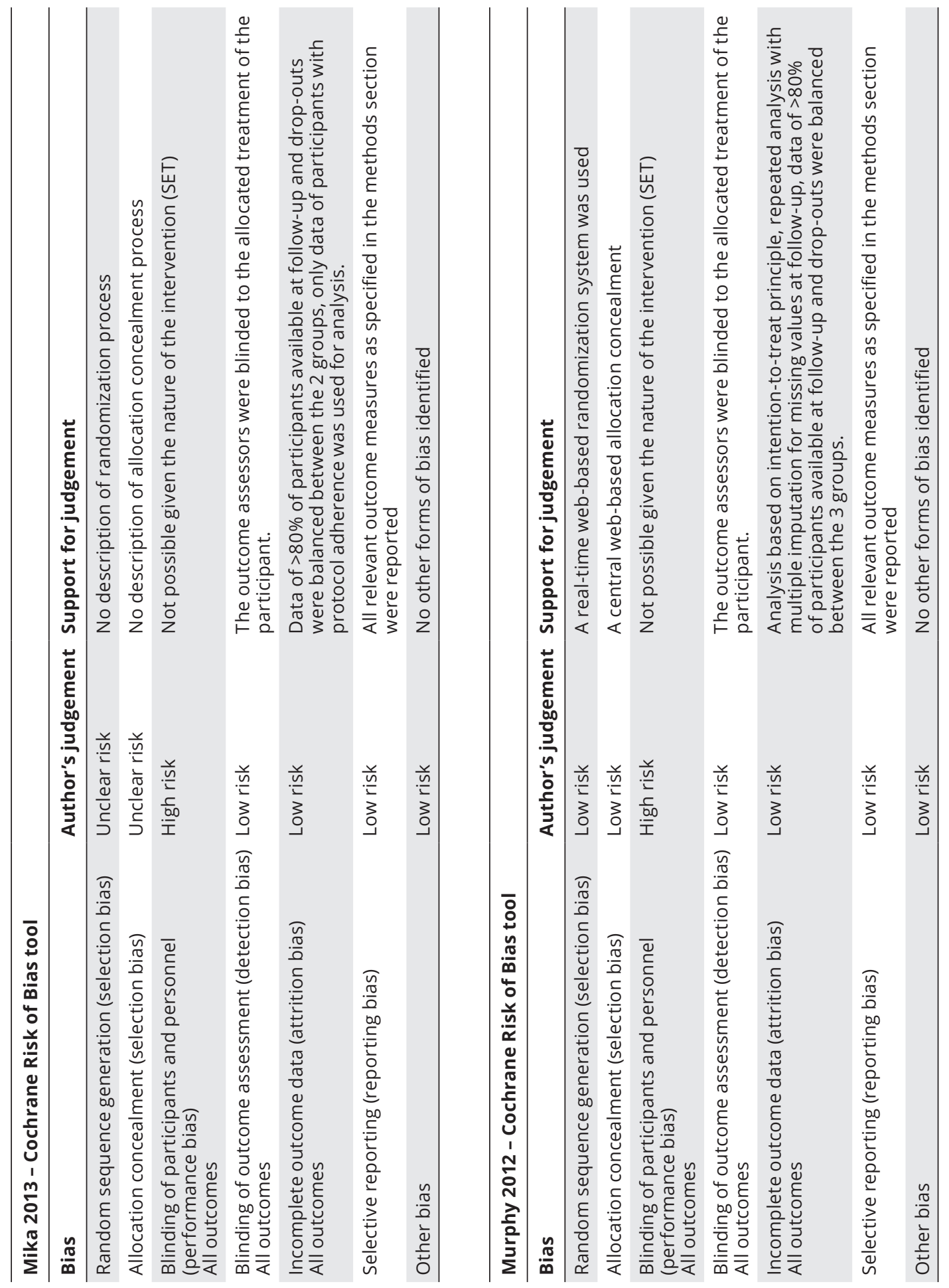

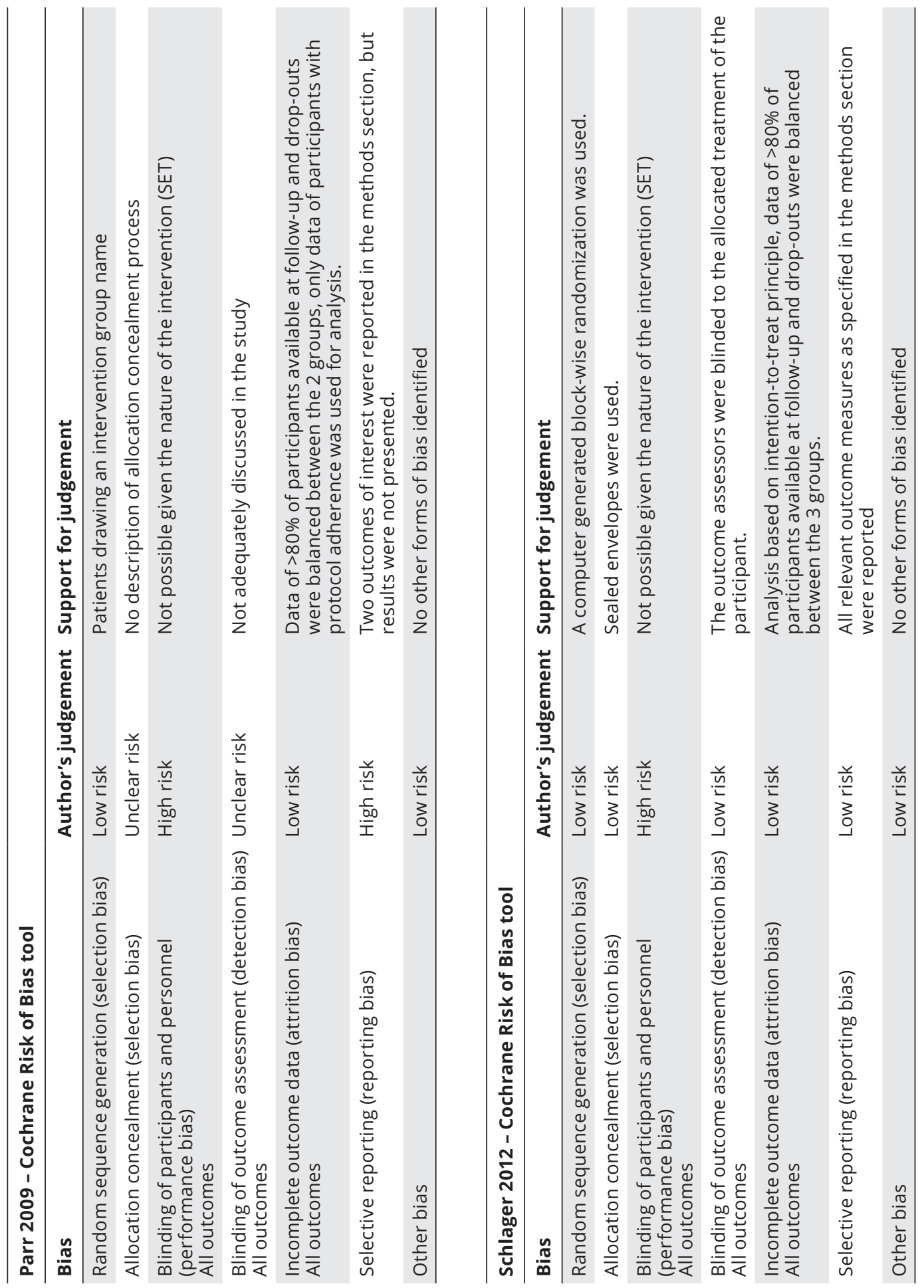


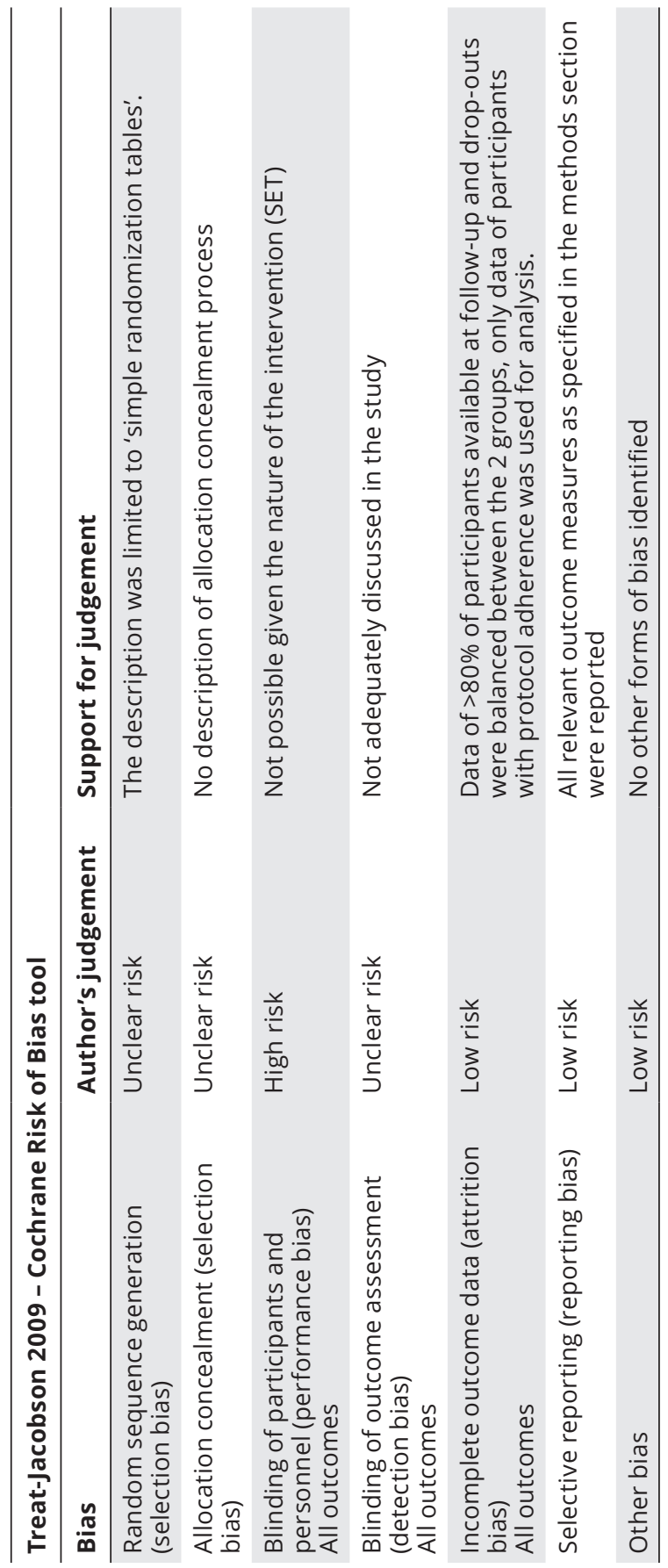




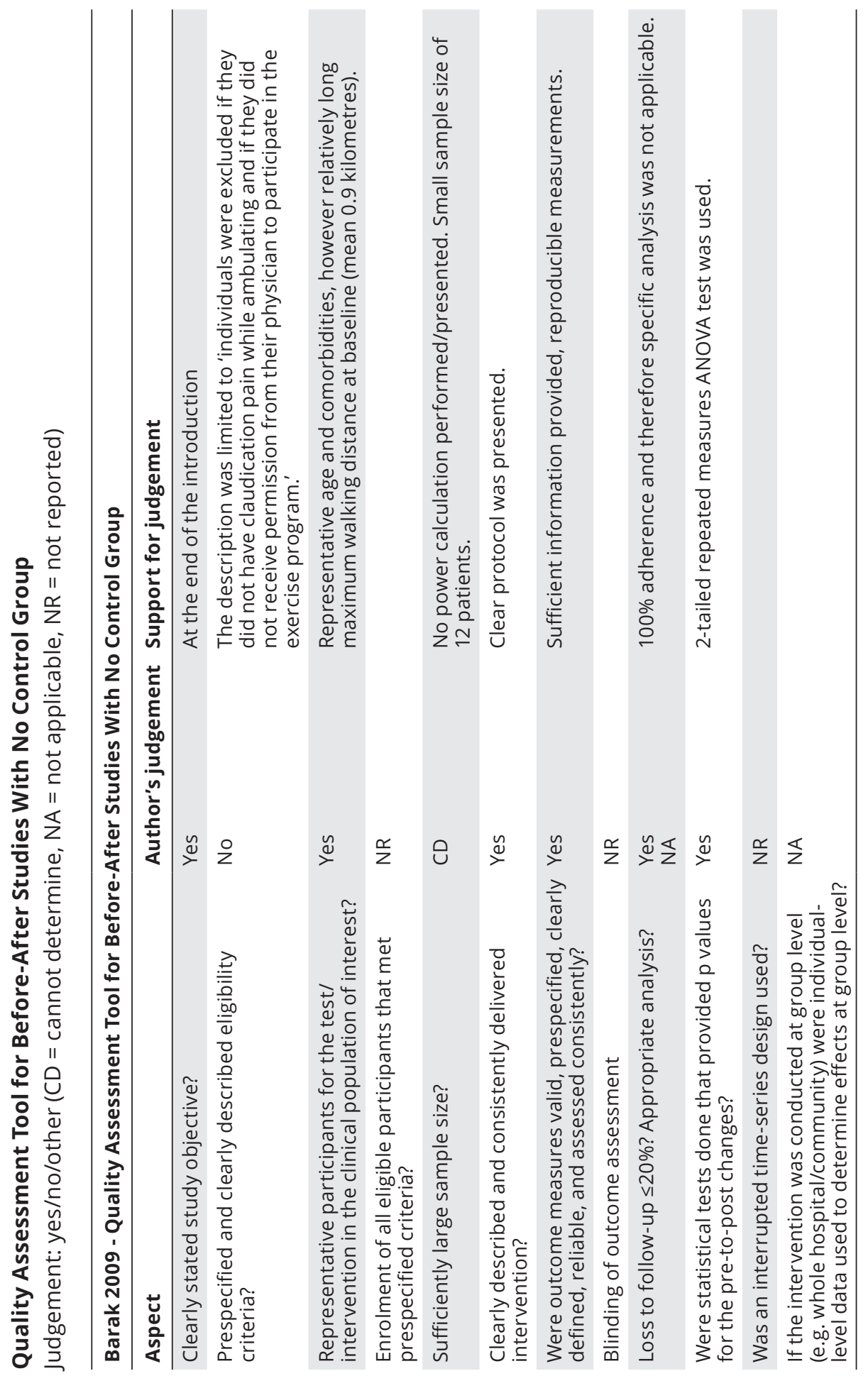




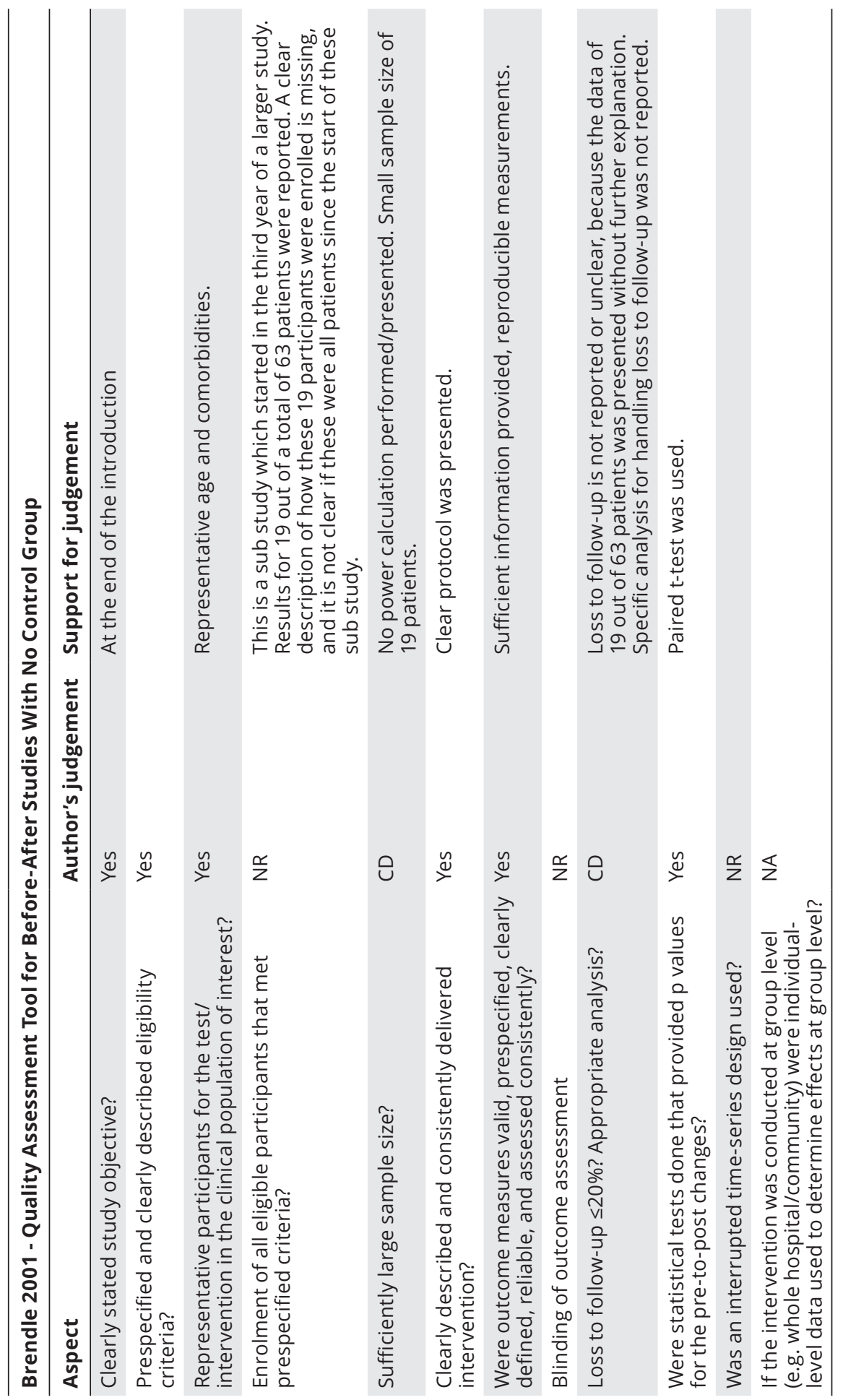




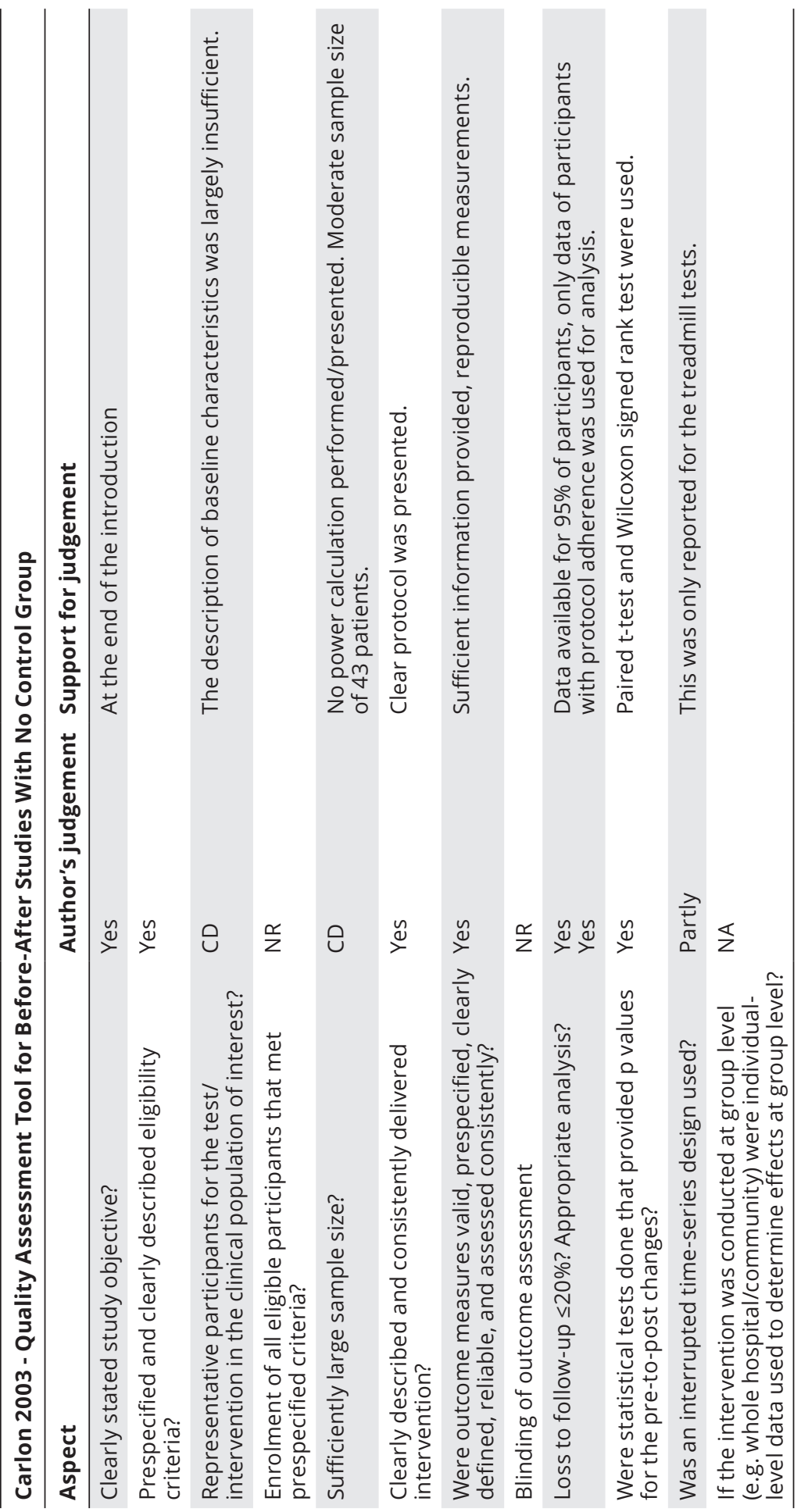




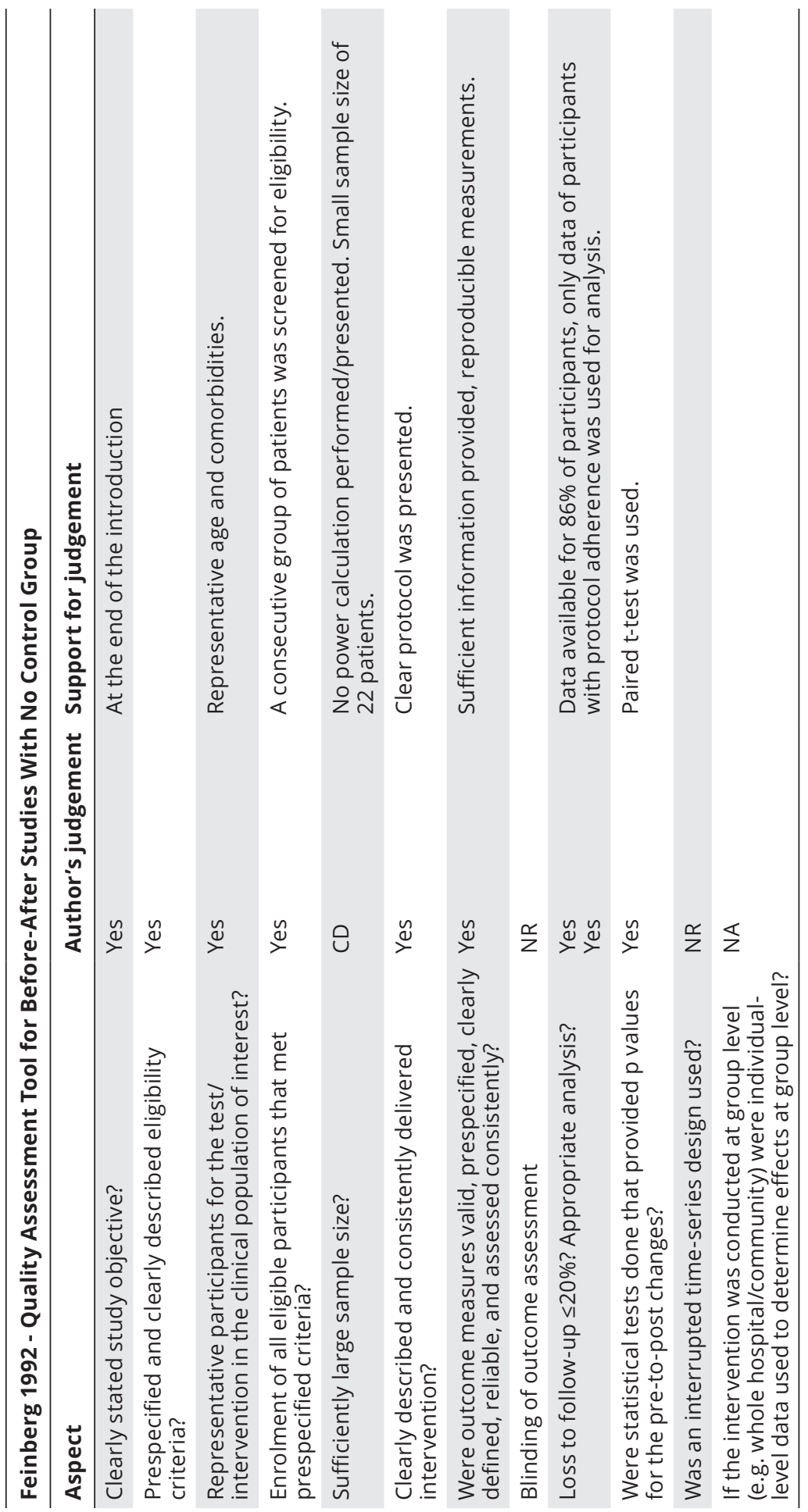




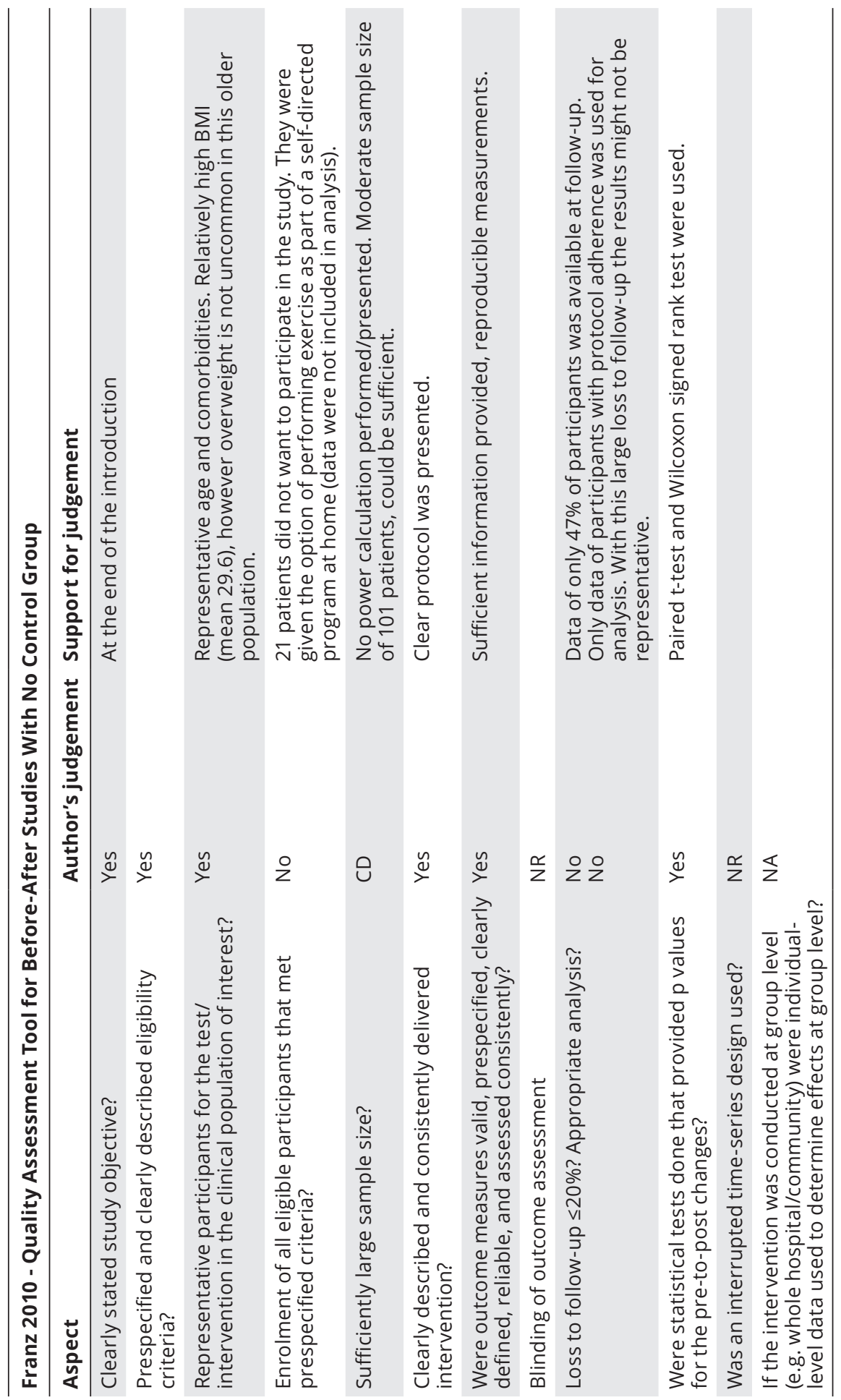




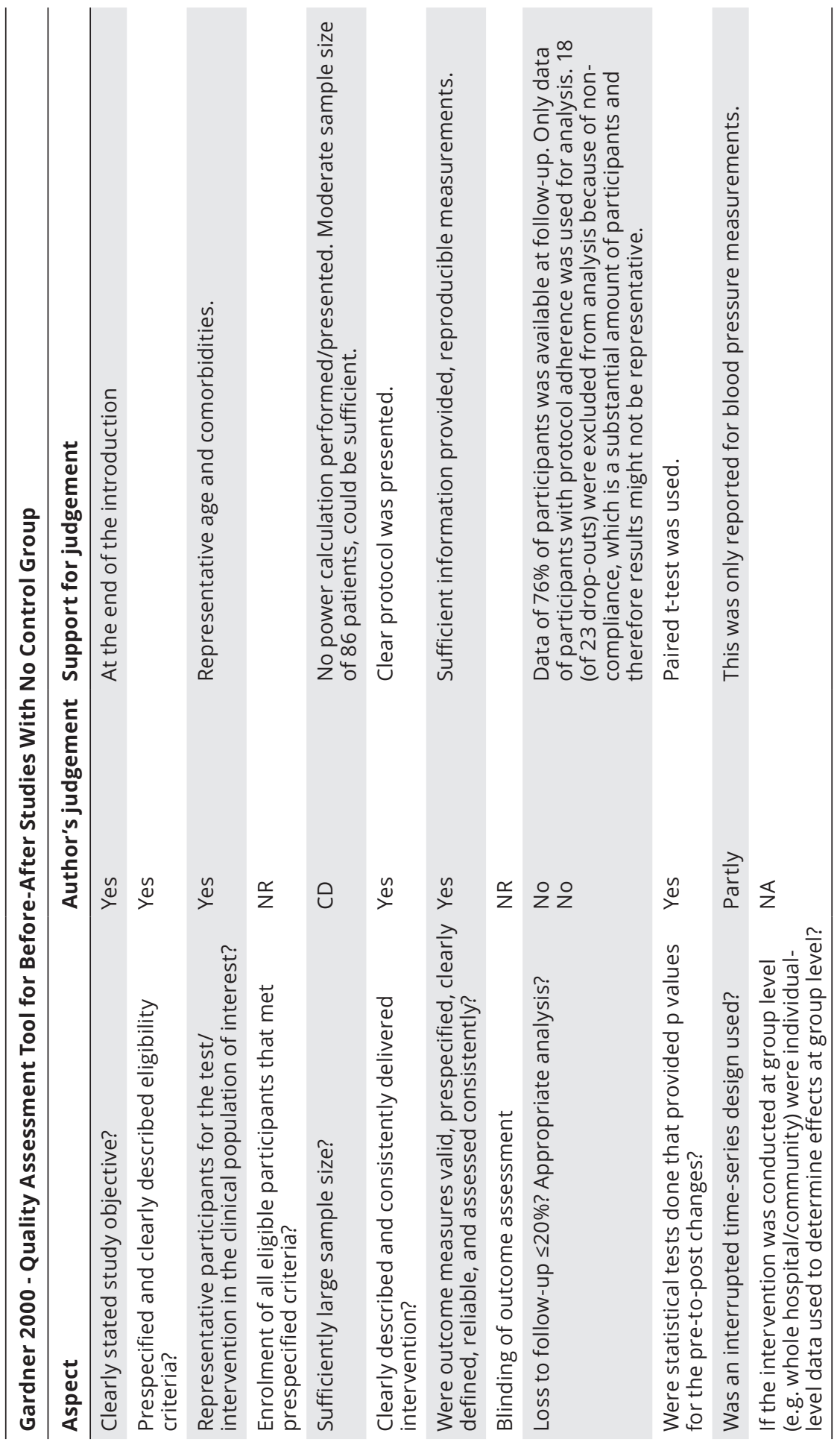




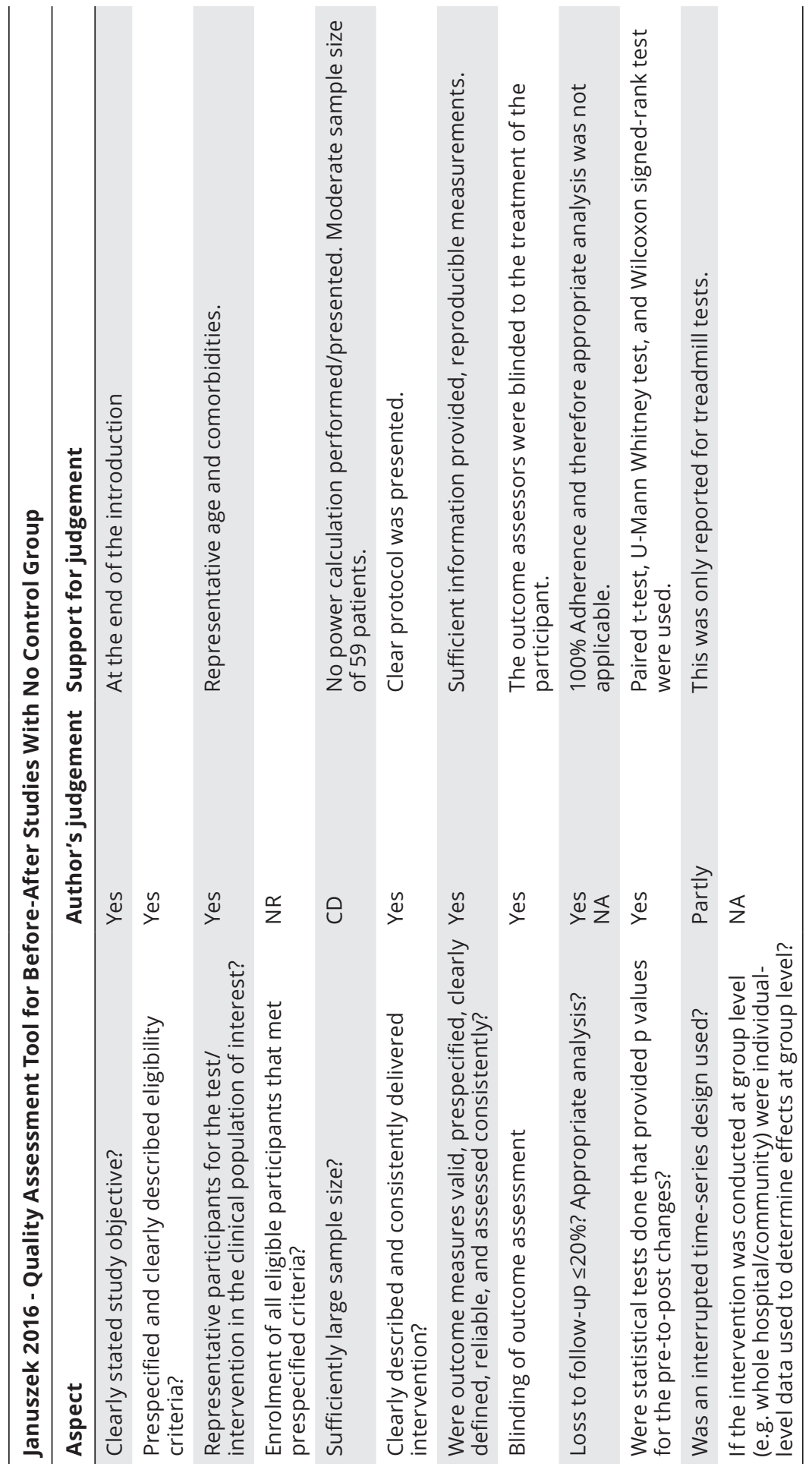




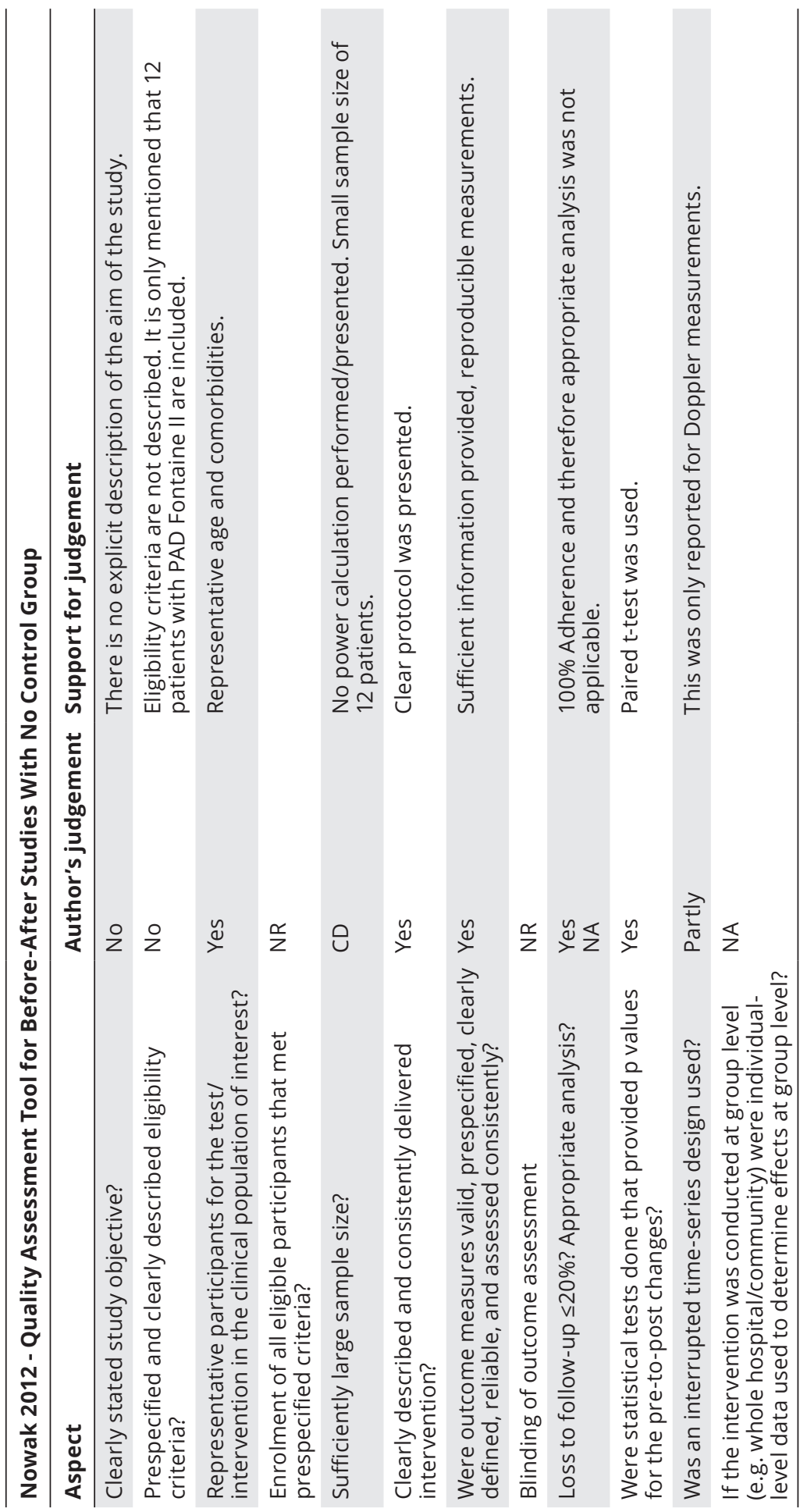




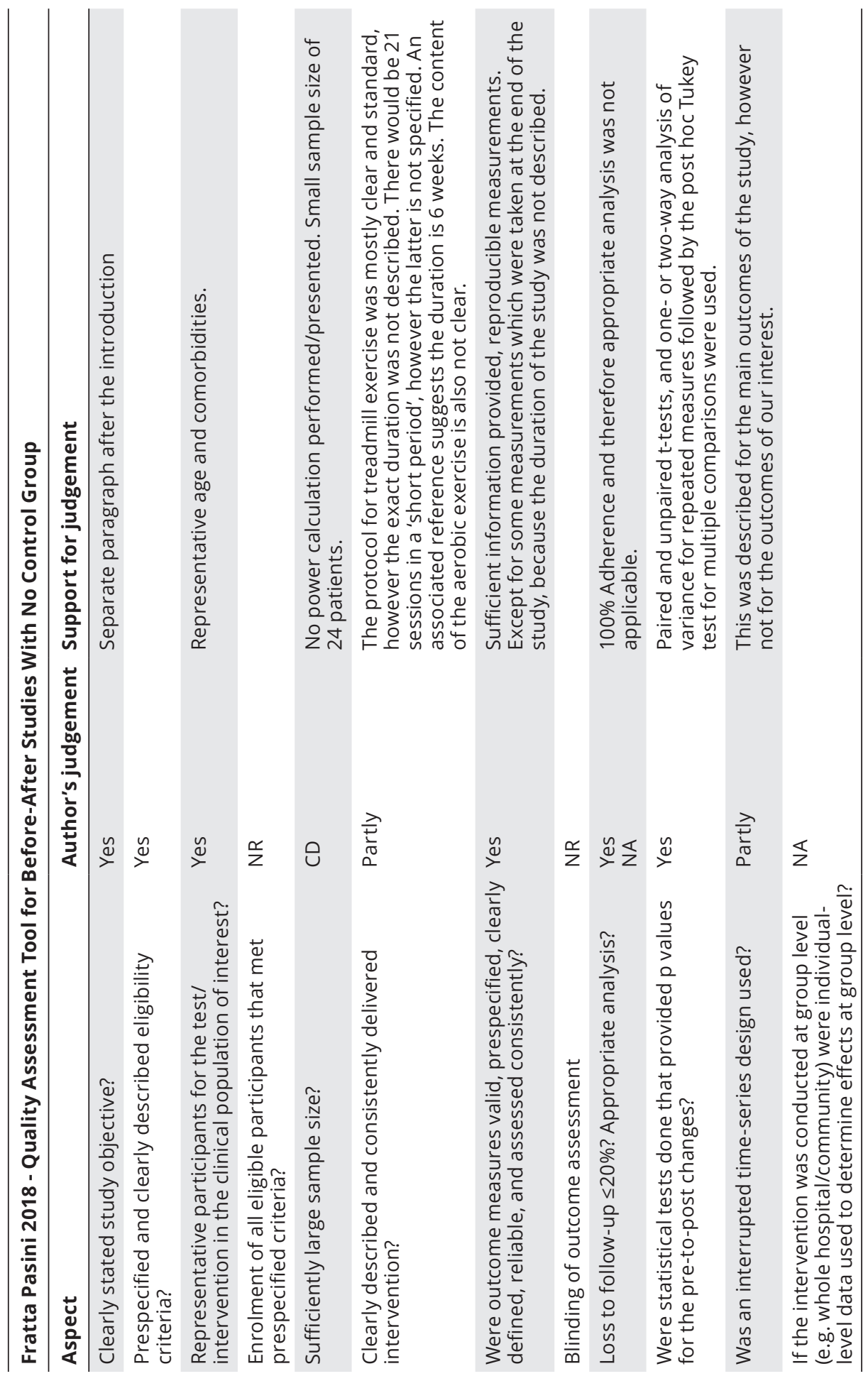




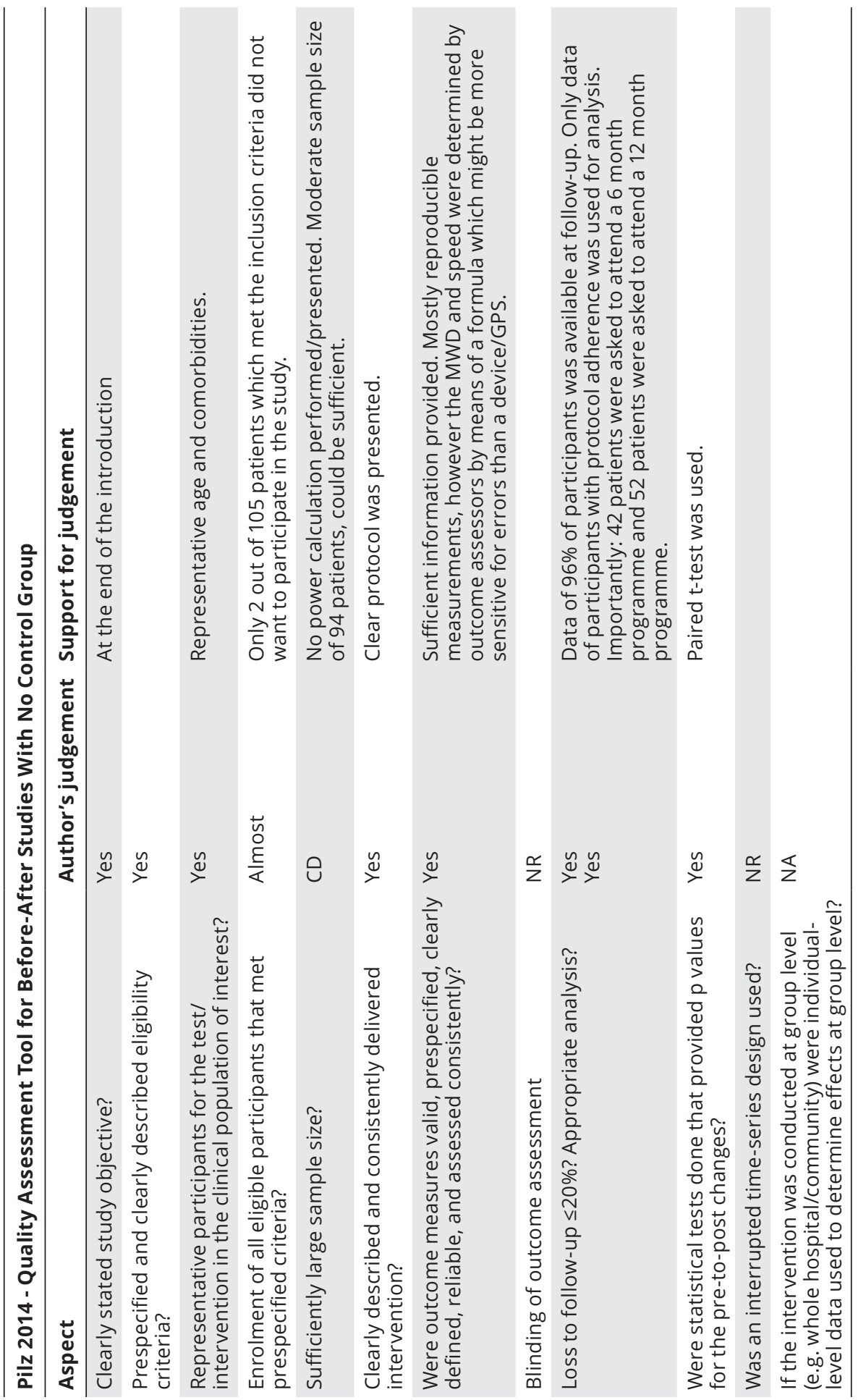




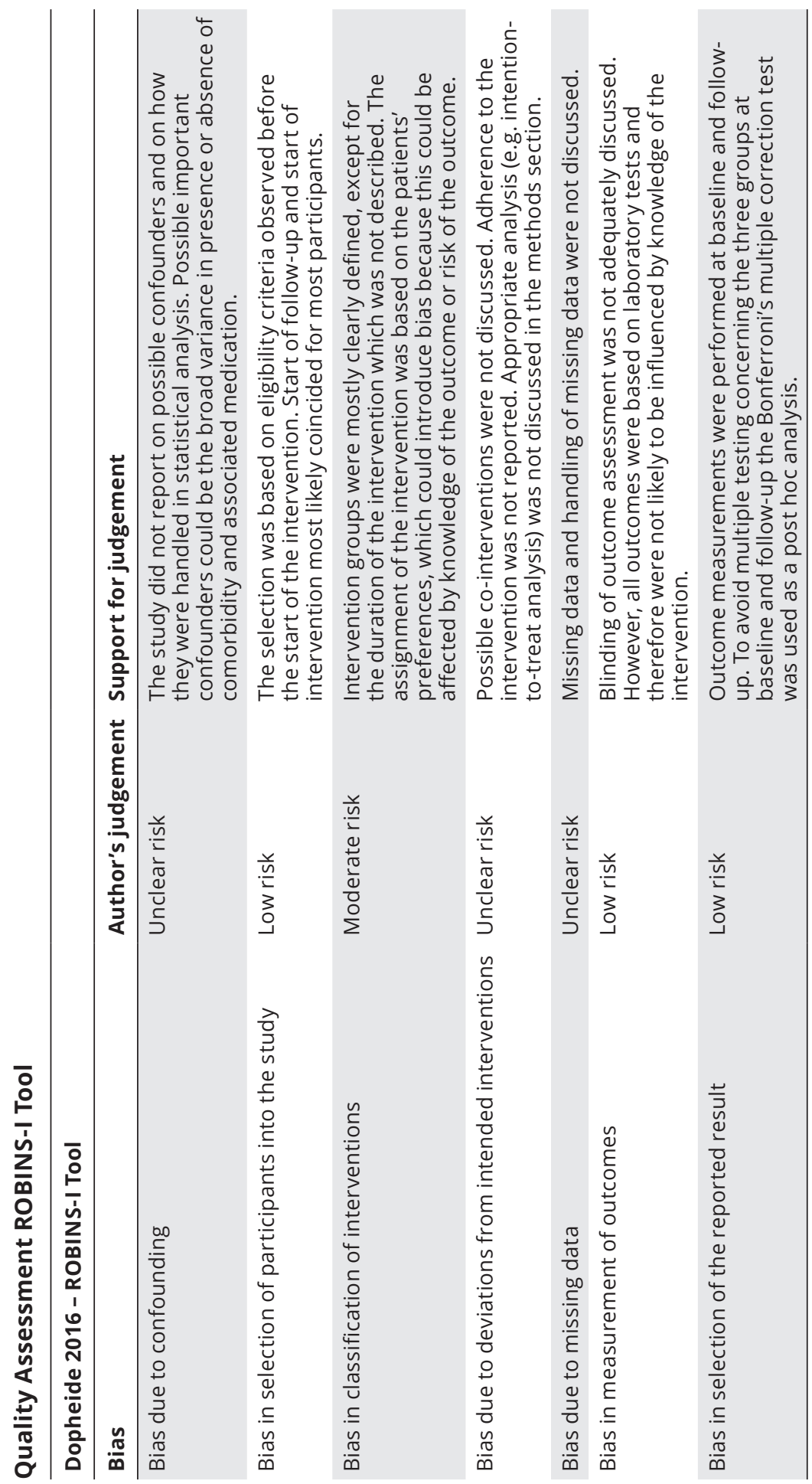




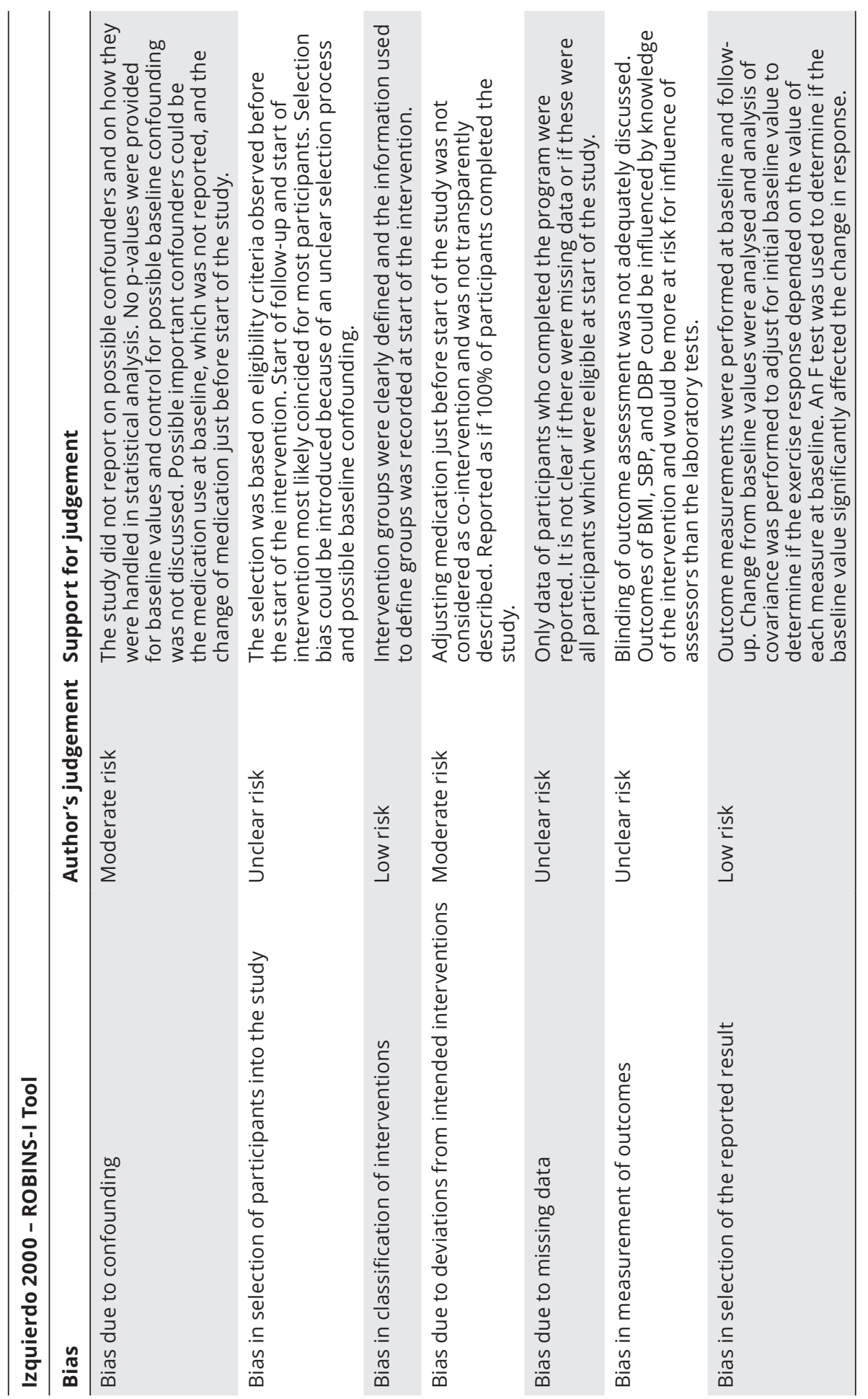



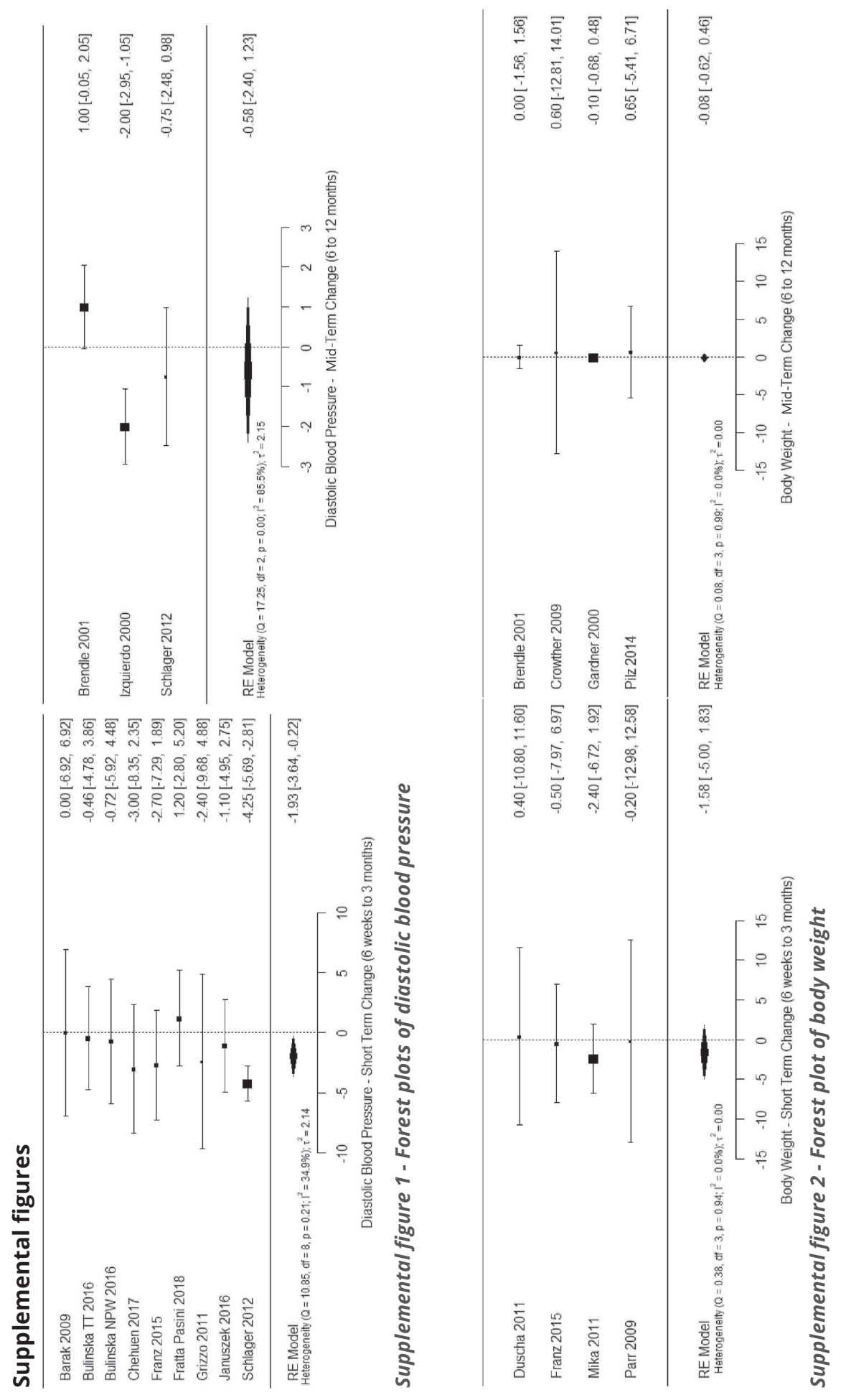

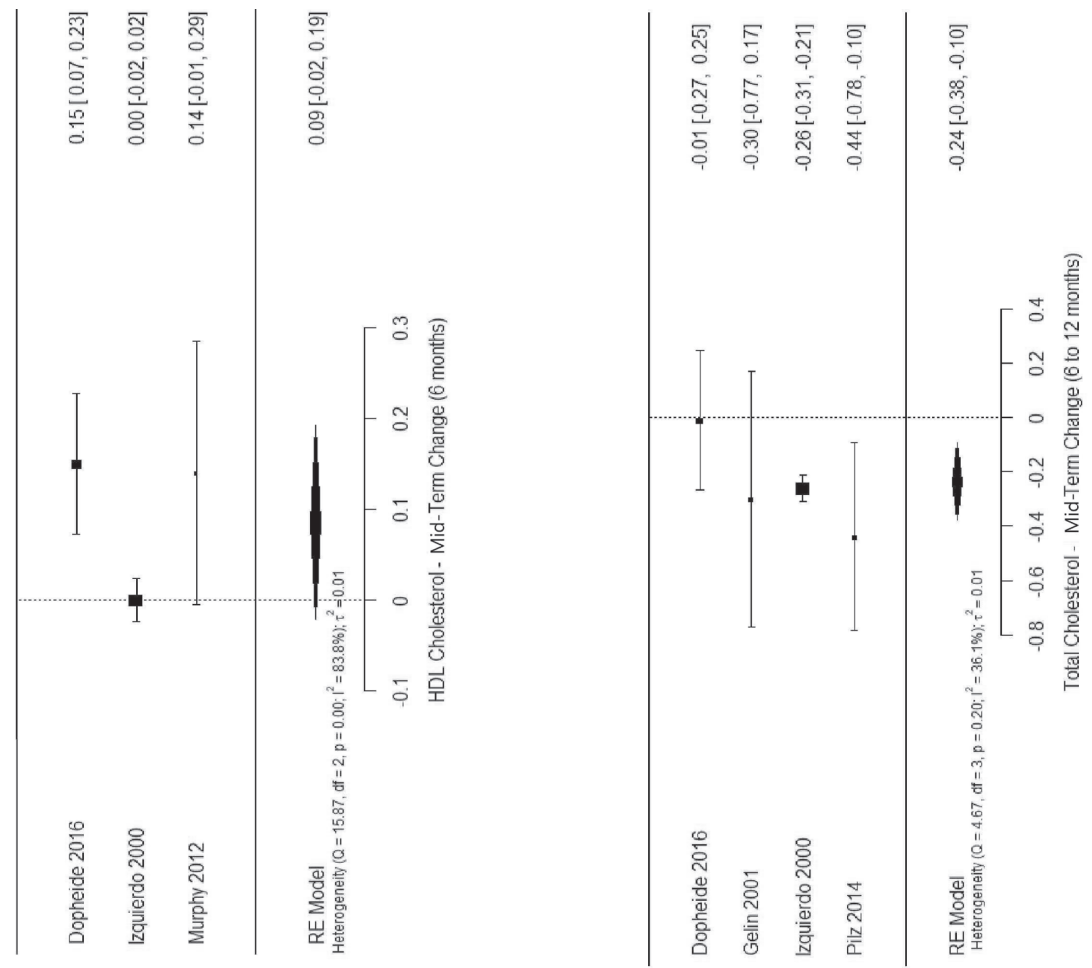

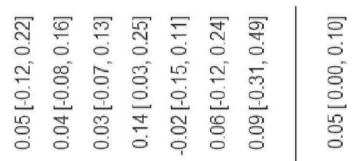

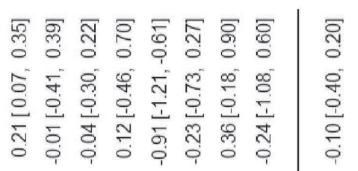
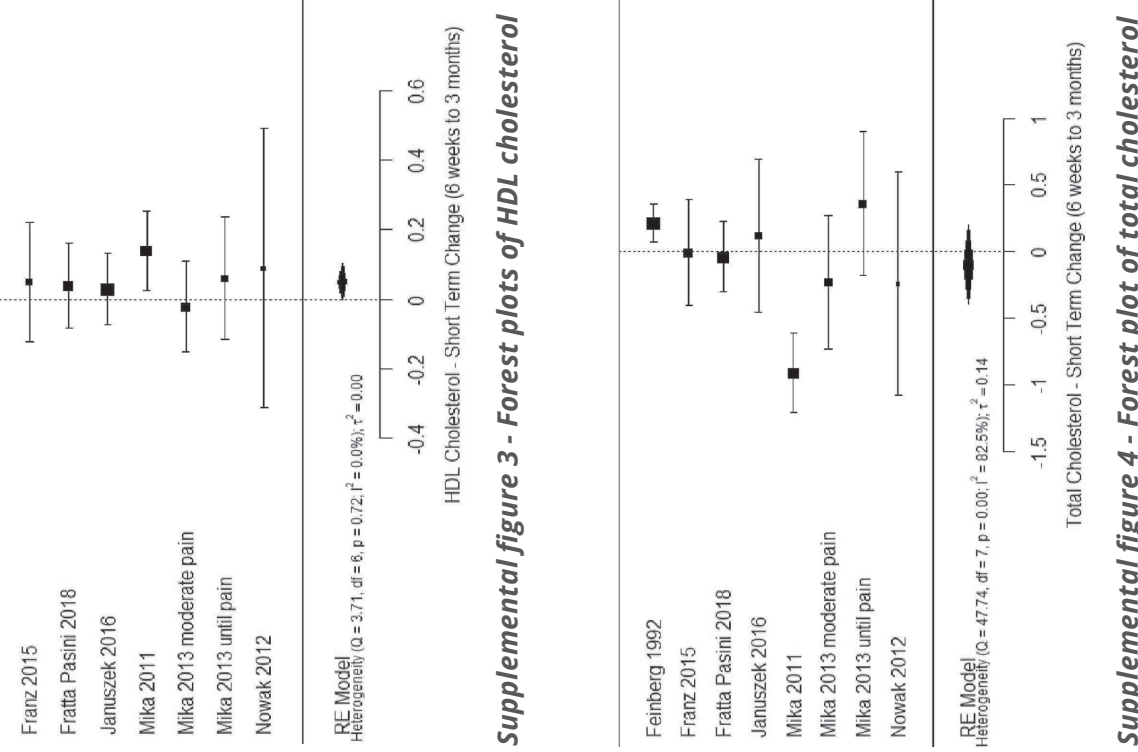

ठे 
chapter 2

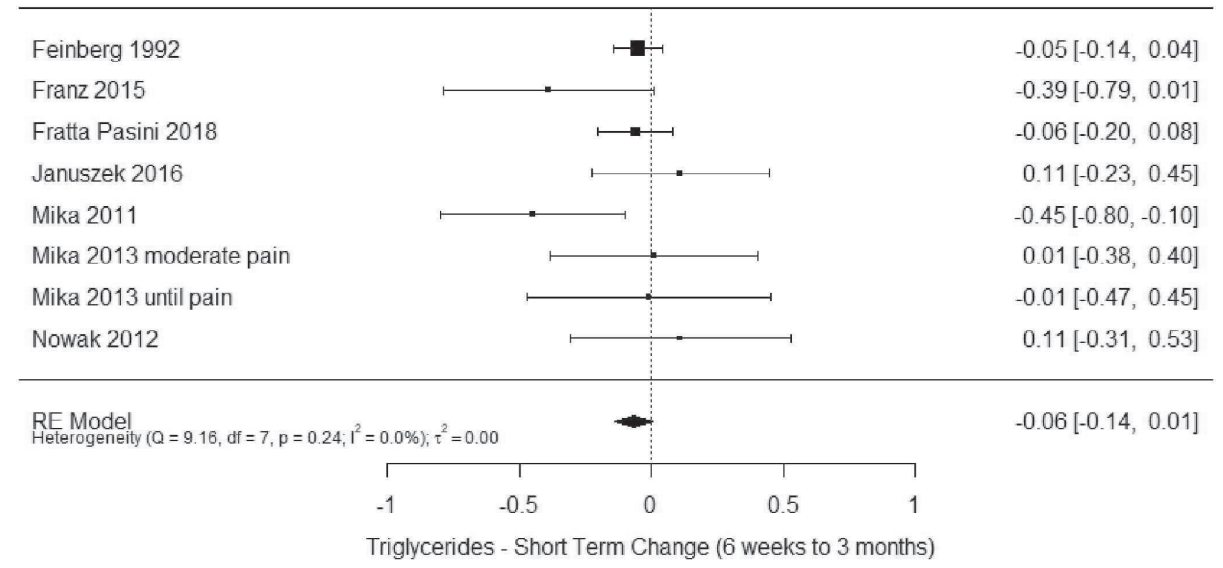

\section{Supplemental figure 5 - Forest plot of triglycerides}

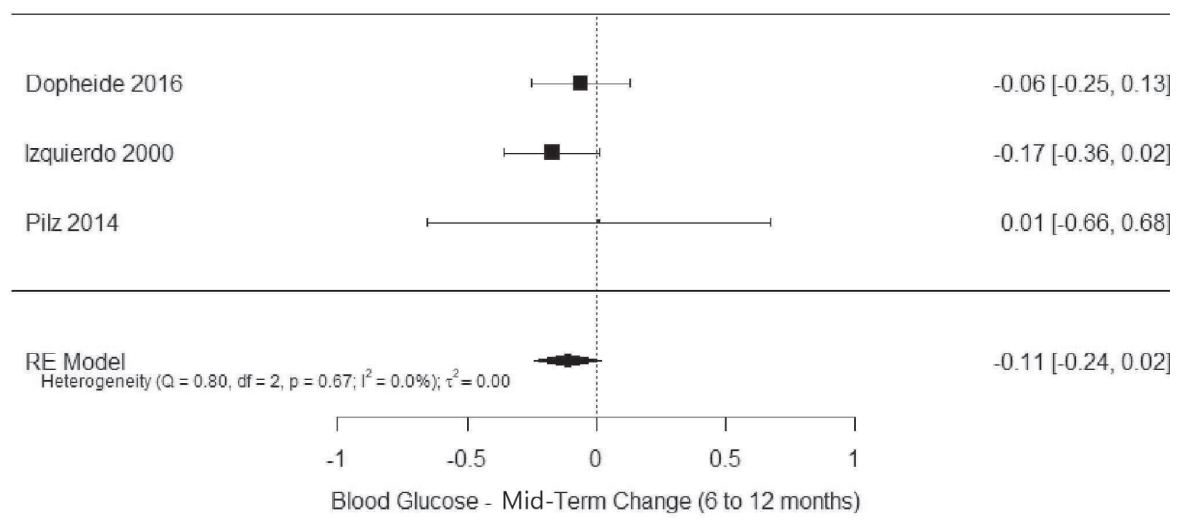

Supplemental figure 6 - Forest plot of blood glucose 



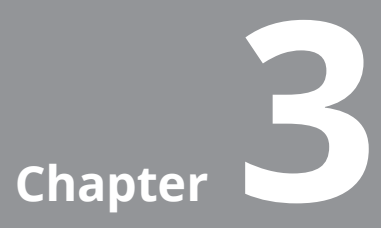

\section{Successful implementation of the exercise first approach for intermittent claudication in the Netherlands is associated with few lower limb revascularisations}

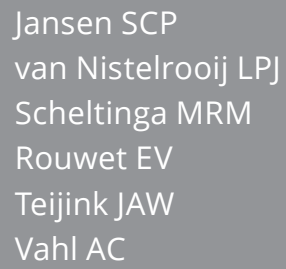




\section{ABSTRACT}

\section{Objectives}

A stepped care model, in which patients are primarily treated with supervised exercise therapy (SET), is recommended as the optimal strategy for intermittent claudication (IC). The aim of this study was to determine the primary treatment (SET, endovascular revascularisation (ER) and open surgery) in relation to secondary lower limb revascularisation and survival in patients with IC.

\section{Methods}

This study is a nationwide retrospective data analysis of health insurance claims of newly diagnosed IC patients between January 2013 and December 2017. Exclusion criteria were the presence of diagnostic codes for critical limb ischemia or for a diabetic foot. Study outcomes were distribution of primary treatment modalities, freedom from secondary lower limb revascularisation, and overall

5 -year survival. Analysis included Kaplan-Meier method and Cox proportional hazards regression models with adjustment for multiple confounders (age, gender, socioeconomic status, use of diabetes medication, statins, platelet aggregation inhibitors or anticoagulants, presence of cardiac disease, chronic obstructive pulmonary disease, and predialysis).

\section{Results}

The 5-year cohort included 54504 IC patients (primary SET $n=39476$, primary ER $\mathrm{n}=11769$, and primary open surgery $\mathrm{n}=3259$ ). SET as primary treatment increased from 63\% in 2013 to $87 \%$ in 2017. Patients who underwent ER or open surgery as a primary treatment had a higher risk of secondary revascularisations (hazard ratio (HR) 1.44; 95\% confidence interval (CI) 1.37 to $1.51 ; p<.0001$ and

HR 1.45; $95 \% \mathrm{Cl} 1.34$ to $1.57 ; \mathrm{p}<.001$, respectively) and a higher mortality risk compared to SET as a primary treatment (HR 1.38; 95\% Cl 1.29 to $1.48 ; p<.001$ and HR 1.49; $95 \% \mathrm{Cl} 1.34$ to $1.65 ; \mathrm{p}<.001$, respectively).

\section{Conclusions}

Guideline adherence improved to $87 \%$ in Dutch patients with IC. Patients receiving primary SET had fewer lower limb revascularisations and demonstrated better survival than patients undergoing primary ER or open surgery. 


\section{INTRODUCTION}

International guidelines on peripheral artery disease (PAD) recommend a stepped care model (SCM) as the optimal treatment strategy for intermittent claudication (IC). A SCM implies that endovascular treatment and surgery are restricted to patients who do not sufficiently benefit from supervised exercise therapy (SET). In addition, revascularisation can be proposed, alongside with SET, if daily life activity is severely compromised. ${ }^{1-3}$ However, implementation of SCM is hampered by suboptimal availability of specialised physiotherapists, referral patterns, and the lack of reimbursement of SET in most countries. ${ }^{4,5}$ Ultimately, the chosen treatment is agreed upon in a shared decision making environment between the care provider and patient.

ClaudicatioNet was founded in 2011 in the Netherlands as a means to improve SCM adherence. ${ }^{6}$ This unique nationwide network of specialised physiotherapists, vascular surgeons, and general practitioners (GPs) offers an innovative organisational structure that guarantees optimal accessibility and quality of SET for IC patients. Previous studies analysed the economic impact of SCM implementation in the Dutch healthcare system using data of one large health insurance company. ${ }^{7,8}$ Compared to the year 2009, a 22\% increase in SCM adherence was demonstrated in 2011, with $56 \%$ of IC patients receiving SET as the primary treatment modality. This improved adherence was associated with $6 \%$ lower average costs of IC treatment per patient.

The current study is a collaboration of ClaudicatioNet and the National Health Care Institute (in Dutch: Zorginstituut Nederland), which is a public organisation that assesses quality, accessibility, and cost-effectiveness of health care in the Netherlands, and advises the Dutch Ministry of Health, Welfare and Sport. This extensive evaluation will contribute to the 'Appropriate Care' project (in Dutch: Zinnige Zorg) that aims to improve the implementation of the SCM in IC treatment to reduce the number of unnecessary invasive interventions and related healthcare costs. ${ }^{9}$ It was hypothesized that higher rates of SCM adherence over a 5-year time period would result in lower rates of lower limb revascularisations. Additionally, this study aimed to determine whether there were differences in overall survival after different primary treatment methods. 


\section{METHODS}

This study was performed according to the STROBE Statement. ${ }^{10}$

Health insurance is obligatory for all Dutch residents. Healthcare activities related to a diagnosis or treatment therefore mandatorily result in healthcare claims. This study is a retrospective analysis of three national claims databases, namely

1. ZPD database (Healthcare Performance and Declarations) that encompasses all reimbursement data from all Dutch health insurance companies, including hospital-based data and community-care based data (i.e. care provided by physiotherapists and general practitioners (GPs)). The mortality data was also retrieved from this database. Source: National Healthcare Institute, data provided by Vektis (data warehouse).

2. DIS database (DBC Information System) that incorporates claims data for hospital-based healthcare. Source: National Healthcare Institute, data made available by Dutch Healthcare Authority (NZA).

3. GIP database (Medicine Information Project) that assembles data on medication use. Source: National Healthcare Institute / GIP.

The combination of these three databases resulted in an accurate compilation of all accorded claims on diagnostic and therapeutic care in all Dutch patients with intermittent claudication within the selected timeframe. The National Health Care Institute is a public organisation that has legal basis and permission for conducting analyses on the databases used in this study. Hence, additional approval from a medical ethical committee was not requested.

\section{Eligibility criteria}

Patients were included if they were diagnosed and treated for IC (i.e. symptomatic PAD Fontaine stage II or Rutherford stages 1-3), defined as the combination of symptomatic PAD specific diagnostic codes, Doppler ultrasound and/or imaging codes (duplex ultrasound, CT-angiography, MR-angiography), and treatment codes (SET, endovascular revascularisation or open surgery). The analysis was restricted to data that had entered the databases between January 2013 and December 2017. Data in national claims databases are obtained retrospectively, which can cause a delay up to 3 years. This is a given phenomenon due to administration issues. Therefore, the analysed data of 2016 and 2017 were $90 \%$ and $80 \%$ complete, respectively. The authors considered these datasets including 2017 reliable and sufficiently complete, since the limited number of missing data was random and proportional. Only newly diagnosed IC patients were eligible, defined as the absence of diagnostic or therapeutic care claims data related to IC in the 3 years prior to 2013. Exclusion criteria were the presence of the diagnostic codes for critical limb ischemia or for a diabetic foot. Please be referred to the supplemental content for in-depth argumentation on the choices made regarding the methodology of this study. 


\section{Definition of groups and specific outcomes}

Patients meeting inclusion criteria were divided into three groups based on primary treatment claims.

- $\quad$ SET group: community-based SET as primary treatment;

- $\quad$ ER group: endovascular revascularisation (ER) including percutaneous transluminal angioplasty with or without stenting as primary treatment;

- $\quad$ OS group: open surgery (OS) including bypass surgery and endarterectomy as primary treatment.

A secondary intervention was defined as lower limb revascularisation following primary treatment and included secondary ipsilateral intervention, primary contralateral intervention, and revision of OS. The available data did not allow for further differentiation between these three groups. According to this definition, a secondary intervention for the SET group was a first revascularisation, whereas it was a second revascularisation for ER and OS groups.

\section{SET programme}

SET typically contains up to 37 individual sessions of 30 minutes during a 3 to 12 months period. The main part of the program consists of treadmill-based or trackbased exercise until the patient experiences claudication symptoms of moderate severity. An exercise-rest-exercise cycle is repeated several times per session.

\section{Outcome measures}

The study objectives were to determine

1. The relative distribution of SET, ER, and OS as the primary treatment for patients with IC between 2013 and 2017.

2. The freedom from secondary interventions according to the primary treatment over a 5-year period.

3. The overall survival following the primary treatment over a 5-year period.

\section{Data analysis}

Patient characteristics were compared using one-way analysis of variance (ANOVA) for continuous variables and the Chi-Square test for categorical variables. Freedom from secondary interventions and overall survival were assessed using the KaplanMeier method. The follow-up period was set at 5 years post intervention. Patients without a secondary intervention were censored at the end of the study period or in case of death. For overall survival, patients were censored at the end of the study. The log-rank test was used to compare survival of groups according to the primary treatment. A p value <.01 was considered statistically significant. Cox proportional hazards regression models were used to analyse the association between primary treatment and time to event outcomes (secondary intervention or death) in unadjusted and adjusted models. Results were summarized as hazard 
ratio (HR) estimates with associated 95\% confidence intervals $(\mathrm{Cl})$. The potential confounders included in the multivariable regression analyses were preselected based on literature, a previous pilot study ${ }^{11}$ analysing claims of a large Dutch health insurance company, and availability of the variables in the claims databases. These confounders were age, gender, socioeconomic status based on postal codes (SES; low, middle, and high tertiles), use of diabetes medication, statins, platelet aggregation inhibitors or anticoagulants (vitamin $\mathrm{K}$ antagonists, direct thrombin inhibitors, and direct factor Xa inhibitors; excl. heparin), the presence of cardiac disease (acute or chronic heart failure, stable or unstable angina pectoris, ST segment elevation myocardial infarction (STEMI) and non-STEMI), chronic obstructive pulmonary disease (COPD), and predialysis (eGFR $<30 \mathrm{ml} / \mathrm{min}$ ). Descriptive statistics were used to determine the other objectives. The number of missing values was expected to be very limited, given the type of data used. Since results are therefore not influenced, only complete case analysis was performed. Statistical analysis was performed using SAS Enterprise Guide 6.1.

\section{RESULTS}

The full study cohort included 54504 patients treated for IC (see Figure 1). Additionally, this study identified a large REST group in which 73239 patients had a Doppler ultrasound and/or imaging, but treatment codes were absent. Patients in this REST group either had no diagnosis of PAD, or were diagnosed as having PAD but were left untreated, or were diagnosed as having PAD but received alternative treatment (e.g. 'walking advice'). Since no further details about these patients could be retrieved from the claims database, this REST group was excluded from analysis as predefined by the study criteria (see Supplemental Methods for extra information).

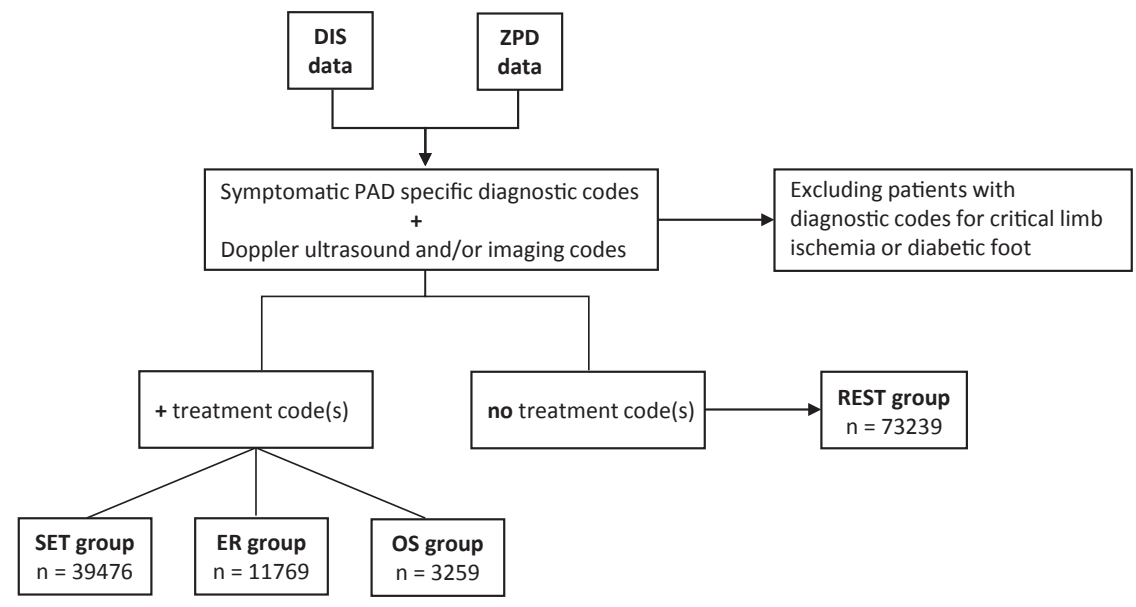

Figure 1 - Flowchart of defined groups

SET supervised exercise therapy, ER endovascular revascularization, OS open surgery 
Patient characteristics and potential confounders are summarized in Table 1 according to their primary treatment. The mean age was 69 years and 59\% were male. Compared to the ER and OS groups, the SET group was 2-4 years older, included more female patients, and more patients used diabetes medication. Furthermore, use of statins, platelet aggregation inhibitors, and anticoagulants were significantly lower in the SET group, and cardiac disease and COPD were less prevalent. The mean length of follow-up was 3 years. Missing data were limited to potential confounders with a maximum of $1.9 \%$.

Table 1. Characteristics of patients with intermittent claudication according to primary treatment between 2013 - 2017 in the Netherlands

\begin{tabular}{|c|c|c|c|}
\hline & SET & ER & os \\
\hline$n$ & 39476 & 11769 & 3259 \\
\hline Age, mean (SD) & $70(10)^{*} \dagger$ & $66(10) \ddagger$ & $68(10)$ \\
\hline Male, n (\%) & $22833(58) * \dagger$ & $7165(61) \ddagger$ & $2361(72)$ \\
\hline Low socioeconomic status, n (\%) & $17055(43)$ *† & $5450(46)$ & $1536(47)$ \\
\hline Use of diabetes medication, $\mathrm{n}(\%)$ & $9968(25) * \dagger$ & $2653(23)$ & $735(23)$ \\
\hline Use of statins, $n(\%)$ & $31769(80) * \dagger$ & $9822(83)$ & $2765(85)$ \\
\hline $\begin{array}{l}\text { Use of platelet aggregation } \\
\text { inhibitors or anticoagulants, } n \\
\text { (\%) }\end{array}$ & $34263(87) *+$ & $10945(93)$ & $3027(93)$ \\
\hline Cardiac disease, n (\%) & $11698(30) * \dagger$ & $3693(31) \ddagger$ & $1167(36)$ \\
\hline COPD, n (\%) & $3803(10) * \dagger$ & $1525(13) \ddagger$ & $477(15)$ \\
\hline Predialysis, n (\%) & $795(2)$ & $213(2)$ & $71(2)$ \\
\hline
\end{tabular}

SET supervised exercise therapy, ER endovascular revascularisation, OS open surgery $* p<0.01$ for SET vs. ER, $t p<0.01$ for SET vs. OS, $\neq p<0.01$ for $E R$ vs. OS

Note: $p$-values were based on one-way ANOVA for continuous variables and Chi-Square test for categorical variables

\section{Distribution of primary treatment modalities}

SET was the primary treatment in the vast majority of patients, with a total of 39476 patients (72\%), followed by ER (22\%) and OS (6\%). Comparison of the years 2013 and 2017 revealed an upward trend of SET as primary treatment from $63 \%$ to $87 \%$ (Figure 2). 


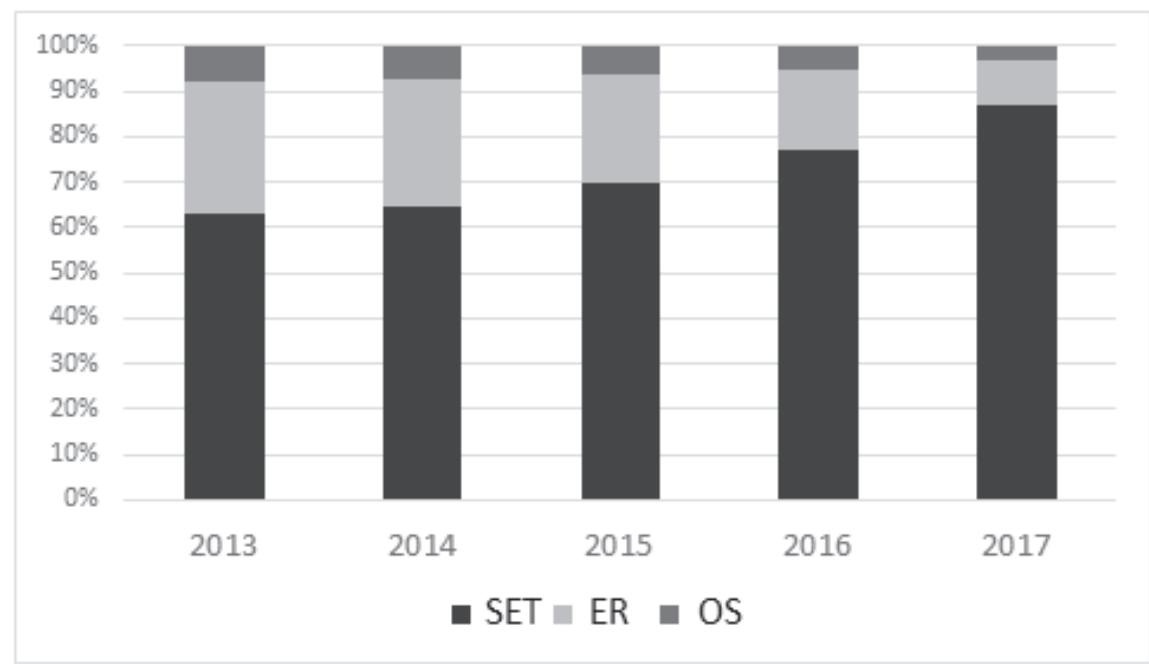

Figure 2 - Distribution of primary treatment modalities 2013 - 2017

SET supervised exercise therapy, ER endovascular revascularization, OS open surgery

\section{Freedom from secondary interventions for IC}

The Kaplan-Meier estimates for the freedom from secondary interventions after 5-year follow-up were 0.8267 (standard error (SE) 0.002) for patients having SET as their primary treatment, 0.7371 (SE 0.005) for ER, and 0.7432 (SE 0.009) for OS (Figure 3). This means that the estimated need for secondary interventions within 5 years was 17 out of 100 patients after primary SET and 26 out of 100 patients for ER and OS. The mean time between primary and secondary intervention was 7 months after primary SET, 6.1 months after ER, and 6.5 months after OS. This analysis included reinterventions done within 30 days after the primary revascularisation, namely in 348 patients (3\%) after primary ER and in 66 patients (2\%) after primary OS. Patients who underwent ER as primary treatment sustained a higher risk of secondary interventions than SET, both in the unadjusted cox-regression model (HR 1.58; 95\% Cl 1.50 to 1.65; $\mathrm{p}<.001$ ) and the adjusted model (HR 1.44; 95\% Cl 1.37 to $1.51 ; p<.0001)$. This was also true for patients who had undergone OS as primary treatment compared to SET in the unadjusted model (HR 1.55; 95\% CI 1.44 to 1.68; $\mathrm{p}<.001$ ) and the adjusted model (HR 1.45; 95\% Cl 1.34 to 1.57; $\mathrm{p}<.001)$. Univariate and multivariable analyses are presented in Supplemental Tables $1 \mathrm{a}$ and $1 \mathrm{~b}$ in the Supplemental Results. 


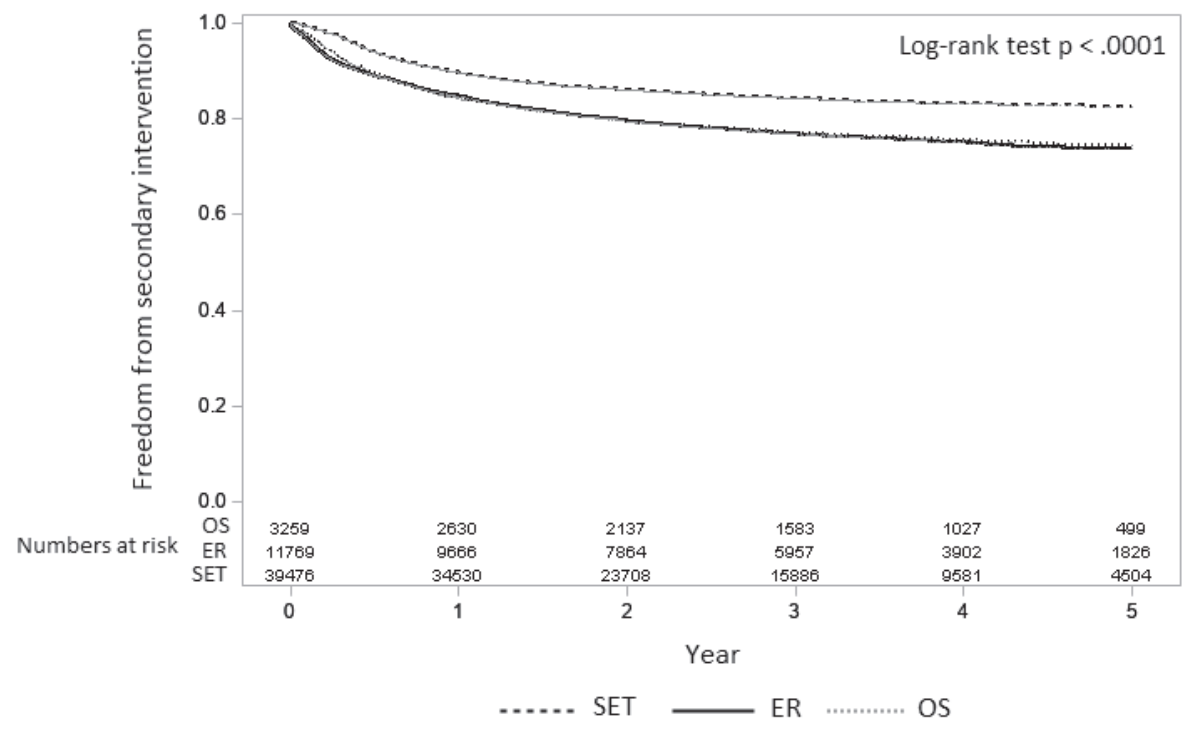

Figure 3 - Freedom from secondary intervention according to primary treatment SET supervised exercise therapy, ER endovascular revascularization, OS open surgery Notable, the graph reflects the first revascularization after SET and the second revascularization for the ER and OS groups.

\section{Overall survival}

The Kaplan-Meier estimates for overall survival after 5-year follow-up were 0.8622 (SE 0.003) for patients who had received SET as the primary treatment, 0.8593 (SE 0.004) for ER, and 0.8187 (SE 0.009) for OS (Figure 4). This means that an estimated 14 out of 100 patients with primary SET or primary ER died within 5 years, and an estimated 18 out of 100 for primary OS. ER as primary treatment was associated with a higher mortality risk compared to SET in the unadjusted coxregression model (HR 1.12; $95 \% \mathrm{Cl} 1.04$ to $1.19 ; \mathrm{p}=.001)$ as well as in the adjusted model (HR 1.38; $95 \% \mathrm{Cl} 1.29$ to $1.48 ; \mathrm{p}<.001)$. The higher mortality risk was also found for OS compared to SET in both the unadjusted model (HR 1.43; $95 \% \mathrm{Cl} 1.29$ to $1.58 ; p<.001)$ and the adjusted model (HR 1.52; $95 \% \mathrm{Cl} 1.37$ to $1.68 ; p<.001)$. Univariate and multivariable analyses are presented in Supplemental Tables 2a and $2 \mathrm{~b}$ in the Supplemental Results. 


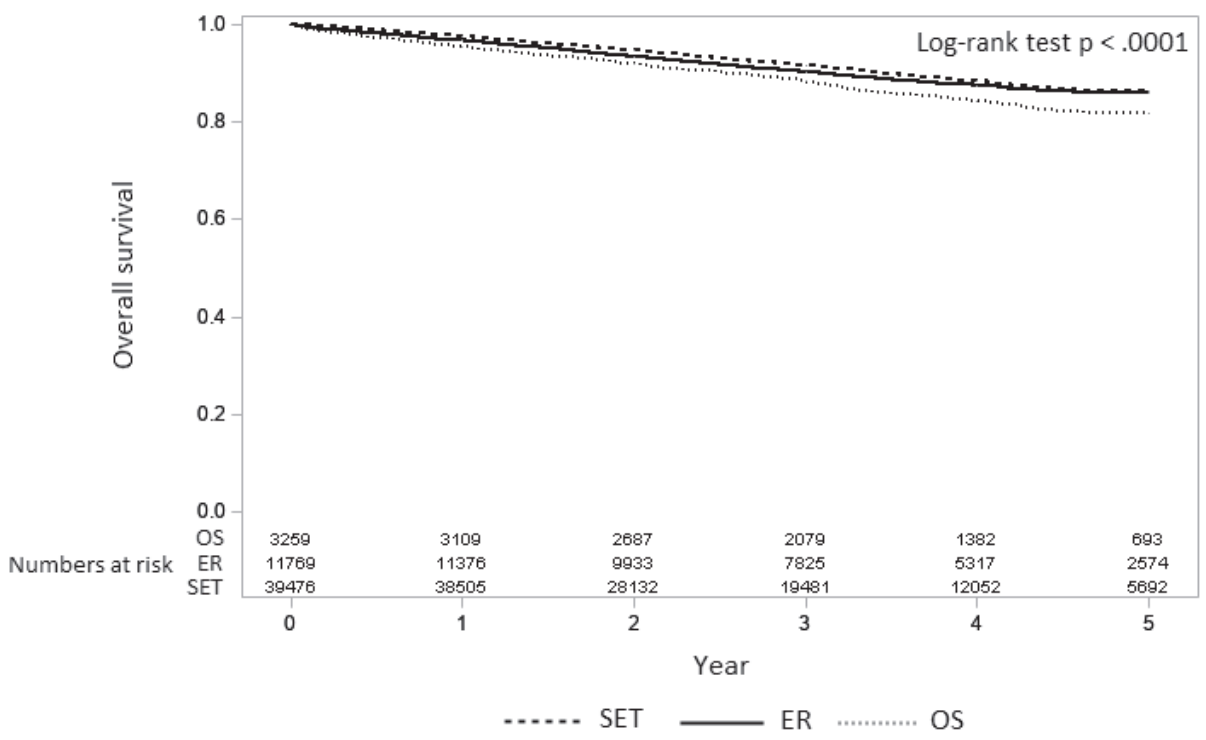

Figure 4 - Overall survival according to primary treatment

SET supervised exercise therapy, ER endovascular revascularization, OS open surgery

\section{DISCUSSION}

This retrospective study of reimbursement data provides a unique insight into realworld nationwide treatment data of 54504 Dutch patients with IC over a 5-year time period. Over time, the guideline adherence to a "SET-first" approach improved to $87 \%$. Interestingly, the 5 -year freedom from revascularisation was $83 \%$ in the primary SET group. Overall, the patients who received SET as primary treatment underwent fewer lower limb revascularisations and had a better 5-year survival than those who were treated with primary revascularisation.

The gradually improved SCM adherence is likely not an automatic consequence of the SET-first approach class I recommendation. ${ }^{1,2}$ Despite abundant evidence supporting benefits of SET, such programs are widely underused in most countries. ${ }^{12}$ Lack of reimbursement and poor patient compliance contribute to this suboptimal participation. 4,5,13,14 The current study reports on the results of IC treatment in a country with sufficient SET availability. Accessibility is optimized through a nationwide organisation of community-based SET provided by specialised physiotherapists as organised by ClaudicatioNet. ${ }^{6}$ Increased awareness of referring physicians also contributed to improved SET referral rates, and offering SET as a full-fledged treatment modality likely decreases patients' reluctance to participate. ${ }^{15}$ The fact that 37 sessions of SET are fully reimbursed since 2017 through basic health insurance (whereas SET was previously only reimbursed through optional 
supplementary health insurance policies) is likely to have further stimulated program participation. Overall, a combined adequate organisation of SET and greater awareness have improved SCM adherence in the Netherlands. Although it is understandable that many researchers focus on alternative treatment options in case of low accessibility of SET, their endeavours should not result in a negligence to improve SET organisation.

A $100 \%$ primary SET rate is not realistic. This is also captured in the guideline recommendations, as primary revascularisation can be proposed if daily life activity is severely compromised., ${ }^{1,2}$ A report of the National Health Care Institute concluded that the number of unnecessary invasive interventions needed to be reduced, based on meanwhile outdated data. ${ }^{9}$ For example, vascular surgeons have been reluctant to prescribe SET in case of cardiopulmonary comorbidity or aortoiliac disease..$^{15}$ The current study demonstrates a successful SCM implementation. Moreover, a primary SET rate of more than $80 \%$ is feasible. Ideally, limited accessibility of SET should not be a factor for favouring revascularisation. Moreover, primary revascularisation should be provided along with SET according to the recommendations. In this study population, only a dismal $10 \%$ received SET in the first year following ER or OS. This finding introduces a new focus for improvement, since enhancing physical capacity should always be included in IC treatment as this is an essential part of cardiovascular risk management.

The current study shows that a "revascularisation first" strategy in IC was associated with a high risk of secondary lower limb revascularisations. Two recent meta-analyses concluded that there was no significant difference in the number of secondary revascularisations at 12 months follow-up in patients who underwent either an endovascular treatment or SET.16,17 These seemingly discrepant findings are possibly explained by the fact that the current study analysed claims data without handling commonly used exclusion criteria regarding comorbidity and lesion specific characteristics, such as unilateral disease or level of disease. In addition, the substantial difference in follow-up (12 months versus 5 years) probably contributed to these different study results. Importantly, we were not able to correct for severity of atherosclerosis, a limitation that can contribute to the observed differences in revascularisation rates. However, it is important to realize that PAD is a manifestation of atherosclerosis resulting in systemic disease. An improved long-term effectiveness is possibly explained by the fact that revascularisation is a focal therapy whereas SET provides systemic benefits. ${ }^{18,19}$

PAD is associated with an increased risk of cardiovascular and all-cause mortality. ${ }^{20,21}$ According to the literature, the 5-year mortality for IC patients is 10 $15 \%$, although these studies may be dated., 22,23 Nevertheless, the mortality rate in this large contemporary cohort is quite similar, suggesting that the efficacy of or adherence to current medications may be limited. As indicated by the medication use in this cohort, there might still be some room for improvement regarding the use of statins (81\%) and platelet aggregation inhibitors or anticoagulants (89\%). ${ }^{1,2}$ 
Moreover, morbidity and mortality risks could possibly be reduced by promoting positive health behaviour. There is abundant evidence on the importance of modifiable risk factors by adapting a healthy lifestyle. A factor that is particularly relevant in IC patients is physical activity. ${ }^{19,24,25}$ In order to optimize results, lifestyle counselling should play a more central role in IC treatment.

Interestingly, a better survival was found in the SET group compared to ER and OS groups. A recent meta-analysis ${ }^{16}$ of studies with up to seven years follow-up found no difference in all-cause mortality when comparing endovascular treatment to SET. On the contrary, the 5-year survival analysis in the current study revealed a survival benefit for those who received SET. However, since we could not adjust for several important determinants of survival in our claims dataset, such as smoking status and ankle-brachial index, these data should be interpreted cautiously. It is possible that SET has a protective effect due to the potential beneficial effects on cardiovascular risk, morbidity, and mortality. ${ }^{19}$ Similar benefits of exercise-based rehabilitation are already well known in cardiac rehabilitation and SET specific evidence is expanding. ${ }^{26}$ An optimal IC treatment strategy should focus on relieving IC symptoms and reducing cardiovascular risk, and should aim for a durable effect.

\section{Limitations}

This retrospective analysis has limitations as the analysed data were restricted to the available national claims. Most importantly, it was not possible to correct for severity of PAD (i.e. walking distance and ankle-brachial indices), which is a potential confounder for all reported outcomes. Additionally, variables such as cigarette smoking and physical activity level, factors known to be associated with overall mortality, were also not available. Because these confounders were not included in the multivariable analysis, it is uncertain whether the observed difference in overall mortality was associated with the primary treatment or caused by residual confounding. In case of a secondary intervention, it was not possible to differentiate between a secondary ipsilateral intervention, a primary contralateral intervention, or a revision of OS. In addition, therapy adherence to SET was unknown. Furthermore, this study could not report results on walking distance and quality of life. However, SET and ER are equally effective in this regard based on current literature. ${ }^{16,17,27}$ It was also not possible to identify combination therapy as a treatment modality. Notably, this upcoming treatment modality of invasive therapy combined with SET is not yet included in the treatment recommendations. Therefore, it is uncertain whether combination therapy was present in the current dataset. As stated in the results section, a large REST group was excluded from analyses. Since we used claims data, it was impossible to retrieve any specifics regarding the patients in this group other than that they were not treated with SET or revascularisation. Despite the mentioned restrictions, this cohort study represents the outcomes of commonly used interventions in a large number of typical patients with IC delivered within 
the context of routine care, in contrast to many trials which offer little insight into adapting treatments in a real-world setting ${ }^{28}$.

\section{CONCLUSION}

SCM adherence improved to 87\% in Dutch patients with IC between 2013 and 2017. Approximately $83 \%$ of patients in the primary SET group remained free of revascularisation up to 5 years of follow-up. Additionally, a new focus for improvement was identified. As only a disappointingly low $10 \%$ received SET in the first year following primary revascularisation, although considered standard care according to the recommendations, future endeavours should be directed towards increasing this rate of participation. 


\section{REFERENCES}

1. Aboyans V, Ricco JB, Bartelink MEL, et al. ESC Guidelines on the Diagnosis and Treatment of Peripheral Arterial Diseases, in collaboration with the European Society for Vascular Surgery (ESVS). Eur J Vasc Endovasc Surg. 2017; 10.1016/j.ejvs.2017.07.018.

2. Gerhard-Herman MD, Gornik HL, Barrett C, et al. 2016 AHA/ACC Guideline on the Management of Patients With Lower Extremity Peripheral Artery Disease: Executive Summary: A Report of the American College of Cardiology/American Heart Association Task Force on Clinical Practice Guidelines. Circulation. 2017;135(12):e686-e725.

3. Layden J, Michaels J, Bermingham S, Higgins B, Guideline Development G. Diagnosis and management of lower limb peripheral arterial disease: summary of NICE guidance. BMJ. 2012;345:e4947.

4. Popplewell MA, Bradbury AW. Why do health systems not fund supervised exercise programmes for intermittent claudication? Eur J Vasc Endovasc Surg. 2014;48(6):608-610.

5. Gommans LN, Teijink JA. Attitudes to supervised exercise therapy. Br J Surg. 2015;102(10):1153-1155.

6. Hageman D, Van Den Houten MML, Spruijt S, Gommans LNM, Scheltinga MRM, Teijink JAW. Supervised exercise therapy: It does work, but how to set up a program? Journal of Cardiovascular Surgery. 2017;58(2):305-312.

7. Fokkenrood HJ, Scheltinga MR, Koelemay $M J$, et al. Significant savings with a stepped care model for treatment of patients with intermittent claudication. Eur J Vasc Endovasc Surg. 2014;48(4):423429.
8. Hageman D, Fokkenrood HJP, Essers PPM, et al. Improved Adherence to a Stepped-care Model Reduces Costs of Intermittent Claudication Treatment in The Netherlands. EurJ Vasc Endovasc Surg. 2017;54(1):51-57.

9. Dutch_National_Healthcare_Institute. Room for improvement analysis: Peripheral Artery Disease. 2016; Available on: https:// english.zorginstituutnederland.nl/ publications/reports/2018/05/30/roomfor-improvement-analysis-claudicationintermittent-zinnige-zorg.

10. von Elm E, Altman DG, Egger $M$, et al. The Strengthening the Reporting of Observational Studies in Epidemiology (STROBE) statement: guidelines for reporting observational studies. J Clin Epidemiol. 2008;61(4):344-349.

11. Internal communication document. Beslisdocument Uitkomstindicatoren Perifeer Arterieel Vaatlijden. Achmea Zorg \& Gezondheid, Kwaliteit van Zorg. December 2013.

12. Treat-Jacobson D, McDermott MM, Bronas UG, et al. Optimal Exercise Programs for Patients With Peripheral Artery Disease: A Scientific Statement From the American Heart Association. Circulation. 2019;139(4):e10-e33.

13. Makris GC, Lattimer CR, Lavida A, Geroulakos G. Availability of supervised exercise programs and the role of structured home-based exercise in peripheral arterial disease. Eur J Vasc Endovasc Surg. 2012;44(6):569-575; discussion 576.

14. McDermott MM. Exercise Rehabilitation for Peripheral Artery Disease: A REVIEW.J Cardiopulm Rehabil Prev. 2018;38(2):63-69. 
15. Hageman D, Lauret GJ, Gommans LNM, et al. Supervised Exercise Therapy for Intermittent Claudication Is Increasingly Endorsed by Dutch Vascular Surgeons. Ann Vasc Surg. 2018;47:149-156.

16. Fakhry F, Fokkenrood HJ, Spronk S, Teijink JA, Rouwet EV, Hunink MGM. Endovascular revascularisation versus conservative management for intermittent claudication. Cochrane Database Syst Rev. 2018;3:CD010512.

17. Pandey A, Banerjee S, Ngo C, et al. Comparative Efficacy of Endovascular Revascularization Versus Supervised Exercise Training in Patients With Intermittent Claudication: Meta-Analysis of Randomized Controlled Trials. JACC Cardiovasc Interv. 2017;10(7):712-724.

18. Lane R, Harwood A, Watson L, Leng GC. Exercise for intermittent claudication. Cochrane Database Syst Rev. 2017;12:CD000990.

19. Jansen SCP, Hoorweg BBN, Hoeks SE, et al. A systematic review and meta-analysis of the effects of supervised exercise therapy on modifiable cardiovascular risk factors in intermittent claudication. J Vasc Surg. 2019;69(4):1293-1308 e1292.

20. Golomb BA, Dang TT, Criqui MH. Peripheral arterial disease: morbidity and mortality implications. Circulation. 2006;114(7):688-699.

21. Criqui MH, Aboyans V. Epidemiology of peripheral artery disease. Circ Res. 2015;116(9):1509-1526.

22. Criqui $\mathrm{MH}$, Langer RD, Fronek $\mathrm{A}$, et al. Mortality over a period of 10 years in patients with peripheral arterial disease. N Eng/ J Med. 1992;326(6):381-386.
23. Weitz Jl, Byrne J, Clagett GP, et al. Diagnosis and treatment of chronic arterial insufficiency of the lower extremities: a critical review. Circulation. 1996;94(11):3026-3049.

24. Piercy KL, Troiano RP, Ballard RM, et al. The Physical Activity Guidelines for Americans. JAMA. 2018;320(19):20202028.

25. Perk J, De Backer G, Gohlke H, et al. European Guidelines on cardiovascular disease prevention in clinical practice (version 2012). The Fifth Joint Task Force of the European Society of Cardiology and Other Societies on Cardiovascular Disease Prevention in Clinical Practice (constituted by representatives of nine societies and by invited experts). Eur Heart J. 2012;33(13):1635-1701.

26. Fletcher GF, Landolfo C, Niebauer J, Ozemek C, Arena R, Lavie CJ. Promoting Physical Activity and Exercise: JACC Health Promotion Series. J Am Coll Cardiol. 2018;72(14):1622-1639.

27. Murphy TP, Cutlip DE, Regensteiner $J G$, et al. Supervised exercise versus primary stenting for claudication resulting from aortoiliac peripheral artery disease: six-month outcomes from the claudication: exercise versus endoluminal revascularization (CLEVER) study. Circulation. 2012;125(1):130-139.

28. Glasgow RE, Lichtenstein E, Marcus AC. Why don't we see more translation of health promotion research to practice? Rethinking the efficacy-to-effectiveness transition. Am J Public Health. 2003;93(8):1261-1267. 


\section{SUPPLEMENTAL METHODS}

This addendum provides in-depth argumentation on choices made regarding the methodology of this study.

\section{Specific diagnostic codes}

Symptomatic PAD specific diagnostic codes included general practitioners' codes and DBC codes 418 and 442 . Code 442 is used for exclusion of PAD and is often registered by the vascular specialist during the first visit of a patient with leg symptoms referred for evaluation of possible intermittent claudication. Theoretically, code 442 would therefore only be combined with diagnostic codes. Following the diagnostic workup, in some cases of confirmed PAD, it is omitted to re-code the patient to 418 (the diagnostic code for intermittent claudication). The combination of code 442 with PAD specific treatment codes indicates that the patient was actually diagnosed with PAD. This occurred in a small but relevant number of cases. Therefore, code 442 was included as specific diagnostic code, in order to obtain an optimally complete dataset.

Exclusion criteria were the presence of the diagnostic codes for critical limb ischemia (DBC code 419 or 420) or for a diabetic foot (DBC code 432).

\section{REST group}

This study identified a large REST group (73239 patients), defined as the combination of symptomatic PAD specific diagnostic codes, and Doppler ultrasound and/or imaging codes, but the absence of treatment codes. The decision to include code 442 as specific diagnostic code largely affected the size of this REST group, which therefore contained a large portion of patients without arterial disease. It possibly also included several patients that were diagnosed as having PAD but were left untreated, or were diagnosed as having PAD but received alternative treatment (e.g. 'walking advice'). It was not possible to describe the composition of this REST group more clearly as the relevant details were not available due to limitations of the claims data. Given its heterogeneity, the REST group was excluded from analysis as predefined by the study criteria.

\section{Time frame}

The analysis was restricted to data that had entered the system between January 2013 and December 2017. Data prior to 2013 were not deemed complete and reliable, since 2012 was a transitional period for a new code system that became operational. Only newly diagnosed patients were eligible, defined as the absence of diagnostic or therapeutic care claims data related to IC in the 3 years prior to 2013. For SET, a period of 12 months prior to 2013 was applied, because SET claims data were first available from 2012. 


\section{SUPPLEMENTAL RESULTS}

Missing values were limited to multivariable analyses with a maximum of 1,9\% (1017 out of 54504 cases excluded in adjusted analyses), due to missing values for SES, sex and age.

Supplemental table 1a. Univariate analysis for the freedom from secondary interventions after 5-year follow-up.

\begin{tabular}{llll}
\hline & HR & $\mathbf{9 5 \%} \mathbf{C l}$ & p value \\
\hline Age & 0.98 & $0.98-0.99$ & $<.0001$ \\
Sex & 0.87 & $0.83-0.91$ & $<.0001$ \\
Socioeconomic status & 1.00 & $0.98-1.03$ & 0.85 \\
Use of diabetes medication & 0.99 & $0.94-1.04$ & 0.81 \\
Use of statins & 1.20 & $1.14-1.27$ & $<.0001$ \\
Use of platelet aggregation & 1.29 & $1.20-1.39$ & $<.0001$ \\
inhibitors or anticoagulants & & & \\
Heart failure & 0.91 & $0.84-0.98$ & 0.02 \\
Angina pectoris & 1.23 & $1.17-1.29$ & $<.0001$ \\
Myocardial infarction & 1.00 & $0.93-1.09$ & 0.93 \\
COPD & 1.11 & $1.04-1.19$ & 0.001 \\
Predialysis & 0.80 & $0.68-0.95$ & 0.01 \\
\hline
\end{tabular}

HR hazard ratio, Cl confidence interval, COPD chronic obstructive pulmonary disease

Supplemental table $1 \mathrm{~b}$. Multivariable analysis for the freedom from secondary interventions after 5-year follow-up, using SET as reference.

\begin{tabular}{llll}
\hline & HR & $\mathbf{9 5 \%} \mathbf{C I}$ & p value \\
\hline ER & 1.44 & $1.37-1.51$ & $<.0001$ \\
OS & 1.45 & $1.34-1.57$ & $<.0001$ \\
Age & 0.98 & $0.98-0.99$ & $<.0001$ \\
Sex & 0.90 & $0.86-0.94$ & $<.0001$ \\
Socioeconomic status & 1.01 & $0.99-1.04$ & 0.31 \\
Use of diabetes medication & 0.99 & $0.95-1.05$ & 0.86 \\
Use of statins & 1.09 & $1.02-1.16$ & $<.01$ \\
Use of platelet aggregation & 1.26 & $1.16-1.37$ & $<.0001$ \\
inhibitors or anticoagulants & & & \\
Heart failure & 0.92 & $0.85-1.00$ & 0.05 \\
Angina pectoris & 1.23 & $1.17-1.30$ & $<.0001$ \\
Myocardial infarction & 0.91 & $0.84-0.99$ & 0.04 \\
COPD & 1.12 & $1.05-1.20$ & $<.001$ \\
Predialysis & 0.85 & $0.72-1.01$ & 0.07 \\
\hline
\end{tabular}

SET supervised exercise therapy, HR hazard ratio, Cl confidence interval, ER endovascular revascularisation, OS open surgery, COPD chronic obstructive pulmonary disease 
Supplemental table 2a. Univariate analysis for overall survival after 5-year follow-up.

\begin{tabular}{llll}
\hline & HR & $\mathbf{9 5 \%} \mathbf{C l}$ & p value \\
\hline Age & 1.08 & $1.07-1.08$ & $<.0001$ \\
Sex & 0.81 & $0.76-0.86$ & $<.0001$ \\
Socioeconomic status & 0.95 & $0.91-0.98$ & $<.01$ \\
Use of diabetes medication & 1.53 & $1.44-1.62$ & $<.0001$ \\
Use of statins & 0.91 & $0.85-0.98$ & 0.01 \\
Use of platelet aggregation & 1.54 & $1.38-1.72$ & $<.0001$ \\
inhibitors or anticoagulants & & & \\
Heart failure & 3.29 & $3.08-3.52$ & $<.0001$ \\
Angina pectoris & 1.15 & $1.08-1.23$ & $<.0001$ \\
Myocardial infarction & 1.54 & $1.40-1.69$ & $<.0001$ \\
COPD & 1.89 & $1.75-2.03$ & $<.0001$ \\
Predialysis & 2.97 & $2.62-3.37$ & $<.0001$ \\
\hline
\end{tabular}

HR hazard ratio, Cl confidence interval, COPD chronic obstructive pulmonary disease

Supplemental table 2 b. Multivariable analysis for overall survival after 5-year follow-up, using SET as reference.

\begin{tabular}{llll}
\hline & HR & $\mathbf{9 5 \%} \mathbf{C l}$ & p value \\
\hline ER & 1.38 & $1.29-1.48$ & $<.0001$ \\
OS & 1.52 & $1.37-1.68$ & $<.0001$ \\
Age & 1.07 & $1.07-1.08$ & $<.0001$ \\
Sex & 0.76 & $0.71-0.80$ & $<.0001$ \\
Socioeconomic status & 0.94 & $0.91-0.98$ & $<.01$ \\
Use of diabetes medication & 1.32 & $1.24-1.41$ & $<.0001$ \\
Use of statins & 0.82 & $0.76-0.89$ & $<.0001$ \\
Use of platelet aggregation & 1.27 & $1.13-1.43$ & $<.0001$ \\
inhibitors or anticoagulants & & & \\
Heart failure & 2.08 & $1.93-2.24$ & $<.0001$ \\
Angina pectoris & 0.86 & $0.81-0.93$ & $<.0001$ \\
Myocardial infarction & 1.31 & $1.19-1.45$ & $<.0001$ \\
COPD & 1.56 & $1.45-1.68$ & $<.0001$ \\
Predialysis & 1.66 & $1.46-1.88$ & $<.0001$ \\
\hline SET supervised exerise therapy & HR hazard ration & $C 1$ confidence & \\
\hline
\end{tabular}

SET supervised exercise therapy, HR hazard ratio, CI confidence interval, ER endovascular revascularisation, OS open surgery, COPD chronic obstructive pulmonary disease 
the exercise first approach is associated with few lower limb revascularisations 



\section{Suitability of supervised exercise therapy}

in patients with intermittent claudication

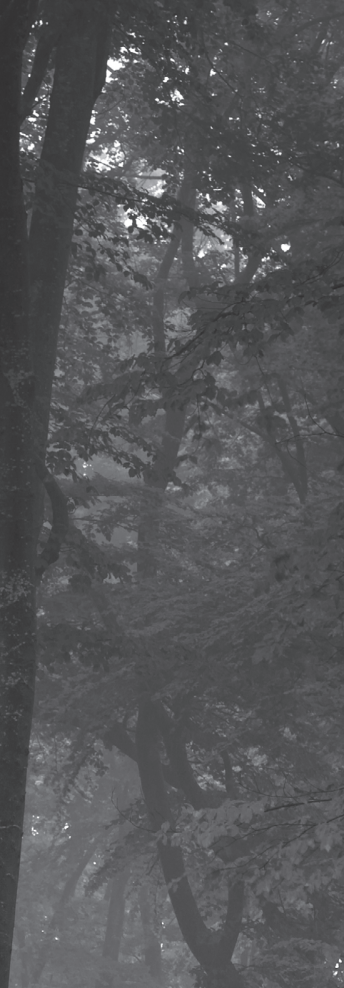





\section{Chapter}

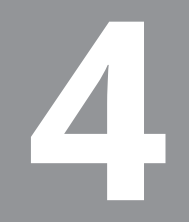

\section{Protocol for a prospective, longitudinal cohort study on the effect of arterial disease level on the outcomes of supervised exercise in intermittent claudication: the ELECT Registry}

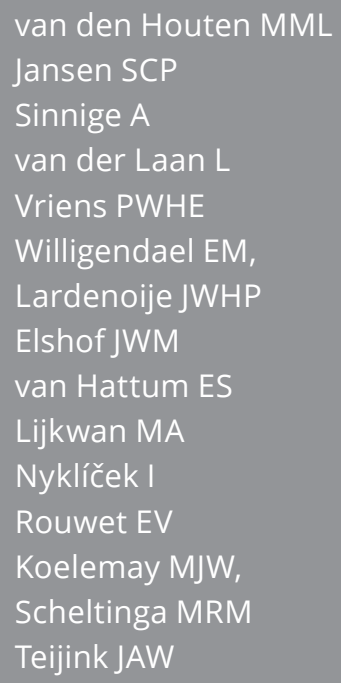




\section{ABSTRACT}

\section{Introduction}

Despite guideline recommendations advocating conservative management before invasive treatment in intermittent claudication, early revascularisation remains widespread in patients with favourable anatomy. The aim of the Effect of Disease Level on Outcomes of Supervised Exercise in Intermittent Claudication Registry is to determine the effect of the location of stenosis on the outcomes of supervised exercise in patients with intermittent claudication due to peripheral arterial disease.

\section{Methods and analysis}

This multicentre prospective cohort study aims to enrol 320 patients in 10 vascular centres across the Netherlands. All patients diagnosed with intermittent claudication (peripheral arterial disease: Fontaine II/Rutherford 1-3), who are considered candidates for supervised exercise therapy by their own physicians are appropriate to participate. Participants will receive standard care, meaning supervised exercise therapy first, with endovascular or open revascularisation in case of insufficient effect (at the discretion of patient and vascular surgeon). For the primary objectives, patients are grouped according to anatomical characteristics of disease (aortoiliac, femoropopliteal or multilevel disease) as apparent on the preferred imaging modality in the participating centre (either duplex, CT angiography or magnetic resonance angiography). Changes in walking performance (treadmill tests, 6 min walk test) and quality of life (QoL; Vascular QoL Questionnaire-6, WHO QoL QuestionnaireBref) will be compared between groups, after multivariate adjustment for possible confounders. Freedom from revascularisation and major adverse cardiovascular disease events, and attainment of the treatment goal between anatomical groups will be compared using Kaplan-Meier survival curves.

\section{Ethics and dissemination}

This study has been exempted from formal medical ethical approval by the Medical Research Ethics Committees United 'MEC-U' (W17.071). Results are intended for publication in peer-reviewed journals and for presentation to stakeholders nationally and internationally.

\section{Trial registration number}

NTR7332; Pre-results. 


\section{INTRODUCTION}

Peripheral arterial disease (PAD) is a chronic condition caused by atherosclerotic narrowing and blocking of the peripheral arteries. Intermittent claudication (IC) is the most common manifestation of PAD and is marked by exertional discomfort in the leg muscles. These symptoms limit walking ability leading to functional disability in daily life. Treatment of IC symptoms aims at improving walking capacity and thereby health-related quality of life (QoL). ${ }^{1}$ Over the past decade, supervised exercise therapy (SET) and endovascular revascularisation (ER) have been shown to be equally effective in this regard. ${ }^{2-8}$ As SET is the non-invasive ${ }^{9}$ and less costly ${ }^{10}$ option, current guidelines recommend SET as primary treatment in the management of patients with IC. ' Ideally, invasive treatment is saved for patients unresponsive to SET, which is the case in approximately $20 \%$ of patients after 2 years. ${ }^{11}$

Clinical practice often deviates from the guidelines, as worldwide reimbursement issues and lacking availability of adequate SET programmes hamper widespread adoption. ${ }^{12-14}$ In the Dutch healthcare system, SET is both available and reimbursed. ${ }^{15}$ Nevertheless, a significant proportion of patients receive early ER in the Netherlands. ${ }^{11}$ Some vascular professionals argue that in certain subsets of patients SET will probably fail and a lower threshold to initiate invasive treatment is warranted. Divergent reasons, oftentimes contradictory, are cited considering factors such as age, comorbidity, (vascular) medical history or personality traits. However, these claims are mostly practice-based as the current literature provides no grounds to discern a subset of patients who will be unresponsive to SET.

One of the main arguments for early revascularisation is the location and extent of the atherosclerotic lesion. Excellent patency rates and procedural results of aortoiliac ER in clinical trials prompt some clinicians to employ more liberal indications to intervene first in these patients. ${ }^{16}$ However, three randomised controlled trials (RCTs) compared SET with ER for patients with IC due to an iliac artery obstruction and found no important differences regarding walking distance or QoL. ${ }^{3517}$ Nonetheless, the idea that in a real-world setting individuals with proximal disease might experience less improvement after exercise training compared with patients with distal lesions remains widespread. Most studies examining the functional outcomes of patients after following a SET programme do not specify the anatomic distribution of disease. Greenhalgh et $\mathrm{al}^{18}$ reported the outcome of SET for aortoiliac and femoropopliteal disease separately, from a trial comparing SET with ER. No formal comparison between outcomes in both anatomic groups was made and the sample size was small, but no apparent difference in effectiveness can be noted. The premise that the outcomes of SET depend on anatomic location and extent of disease is not based on empirical evidence.

Based on the available evidence an inferior effect of SET due to lesion location cannot be assumed. Therefore, the primary aim of this study is to determine the effect of the location of stenosis (femoropopliteal vs aortoiliac vs multilevel disease) 
on the outcomes of SET in patients with IC. To this end, the functional and clinical outcomes from 'real world' subjects treated with SET will be recorded, applying a minimal amount of subject selection criteria.

\section{Research objectives}

\section{Primary objective}

The aim of the Effect of Disease Level on Outcomes of Supervised Exercise in Intermittent Claudication (ELECT) Registry is to determine the effect of the location of stenosis on the outcomes of SET in patients with IC (PAD; Fontaine II, Rutherford 1-3), by recording the clinical outcomes from consecutive 'real world' subjects treated with SET. The primary objective is to determine the outcomes of SET in patients with aortoiliac disease compared with femoropopliteal disease with regard to the following measures:

1. The primary end point is change in maximum and functional walking distance on a standardised treadmill test after 3, 6 and 12 months of SET.

2. Change in 6-minute walk test (6MWT) performance after 3, 6 and 12 months of SET.

3. Change in Vascular QoL Questionnaire-6 (VascuQoL-6) and WHO QoL Questionnaire-Bref (WHOQoL-BREF) outcomes at 3 and 6 months, and 1, 2 and 5 years, follow-up.

4. Freedom from vascular interventions for the lower-extremities, at 6 months and 1, 2, and 5 years, follow-up.

5. Achievement of the main treatment goal, as drafted by the physical therapist and patient at the start of the SET programme, and indicated in the feedback letter after 3, 6 and 12 months of SET.

6. Freedom from major adverse cardiovascular events at 1, 2 and 5 years, follow-up.

\section{Secondary objectives}

This study will also determine the outcomes of SET in patients with aortoiliac disease compared with femoropopliteal disease, multilevel disease and patients without aortoiliac and femoropopliteal disease, with regard to the above-mentioned measures. The ELECT Registry dataset will furthermore be used to investigate the effect of SET on the overall IC population regarding the following objectives:

1. To determine whether several baseline clinical characteristics and functional measures are predictive of changes in walking performance and clinical outcomes of SET.

2. To determine whether specific personality traits (extraversion, neuroticism, conscientiousness, self-control, barrier self-efficacy, anxiety, depression and optimism) measured at baseline are predictive of the clinical outcomes of SET. 
3. To determine the change in barrier self-efficacy after 3, 6 and 12 months of SET.

\section{METHODS}

\section{Study design and setting}

The ELECT Registry is a multicentre prospective cohort study initiated from the vascular surgery department of the Catharina Hospital in Eindhoven, the Netherlands. All patients diagnosed with IC (PAD; Fontaine II/Rutherford 1-3), who are considered candidates for SET by their own physicians, and meet the inclusion criteria (box 1), are eligible. Recruitment will take place in 10 vascular surgery departments throughout the Netherlands: the Catharina Hospital, Amphia Hospital, Elisabeth Twee Steden Hospital, Albert Schweitzer Hospital, Rijnstate Hospital, Medical Spectrum Twente, University Medical Centre Utrecht, Amsterdam University Medical Centre, Máxima Medical Centre and VieCuri Medical Centre. In these centres, the treating vascular surgeon will seek verbal consent from eligible patients to be approached by a research coordinator. Candidates will subsequently receive written information on the study, inviting them to participate, with two consent forms and a self-addressed envelope. These patients will be contacted by telephone by the coordinating investigator within 1 week after their visit to the vascular surgeon to establish formal agreement to participate in the study. If the patient agrees to participate, he/she will sign the consent forms and will send both forms in the self-addressed envelope to the coordinating investigator. After receiving the two consent forms, the coordinating investigator will sign both forms and return one version to the patient. To ensure adequate data collection, the participating centres are recommended to schedule the subjects' follow-up visit(s) based on the current standard of care as prescribed by the Dutch guidelines, which is at 3- 6 months. At this moment, the decision to either continue conservative management, or treat invasively (endovascular or open revascularisation) is generally made. 


\section{Box 1. Eligibility criteria}

\section{Inclusion criteria}

- IC due to unilateral or bilateral PAD (Fontaine II, Rutherford 1-3)

- $\quad$ Resting $A B \mid<0.9$ (or $T B \mid<0.7$ ) or drop in $A B \mid>0.15$ after exercise

- Candidate for SET as a primary treatment, at the discretion of the treating vascular surgeon

- Recent or planned imaging of at least the aortoiliac and femoropopliteal tract (within 6 months of SET initiation, but prior to possible vascular intervention): either colour duplex scanning or CTA or MRA

- $\quad$ Signed informed consent form

\section{Exclusion criteria}

- Advanced PAD beyond IC (i.e. ischaemic rest pain and/or ulcers, Fontaine $>I I$, Rutherford 4-6)

- High probability of non-adherence to physician's or physical therapist's follow-up requirements (e.g. due to lack of motivation or past compliance issues)

- Current participation in a concurrent trial that may confound study results

- Vascular intervention as primary treatment, at the discretion of the treating vascular surgeon

- Prior SET, performed in accordance with the guidelines of the Dutch Society for Physical Therapists, in the previous 12 months

- Neurogenic/venous/orthopaedic claudication more dominant than arterial claudication complaints

$A B I$, ankle-brachial index; CTA, computed tomographic angiography; IC, intermittent claudication; $M R A$, magnetic resonance angiography; $P A D$, peripheral arterial disease; $S E T$, supervised exercise therapy; TBI, toe-brachial index.

\section{Treatment of subjects}

All patients will receive standard cardiovascular risk management by their physician; including smoking cessation advice, statin therapy and platelet inhibitors, as explicated in the multidisciplinary guidelines. ${ }^{1}$ Furthermore, patients will receive a standard regimen of SET, which entails exercise and lifestyle coaching. SET is provided by qualified physical therapists according to usual practice (specified in the physical therapy guidelines). ${ }^{19}$ All therapists are affiliated with ClaudicatioNet, a Dutch network of physical therapists specialised in SET with lifestyle guidance. This guarantees uniform quality of care through mandatory training courses in practice guidelines, motivational interviewing skills and other IC-relevant topics. ${ }^{15} \mathrm{~A}$ typical SET programme contains up to 37 individual sessions, spanning 3-12 months. A session consists of 30 min of treadmill-based or track-based exercise. The initial workload of the treadmill is set to a speed and grade that elicits claudication symptoms within 3-5 min. Patients are asked to continue to walk at this workload until they experience claudication of moderate severity. A brief period of rest permits symptoms to resolve. An exercise-rest-exercise cycle is repeated several times. Such a programme requires intense monitoring of patients aimed at increasing workload by adjusting treadmill grade or speed (or both)..$^{15} 19$ This will generally be performed at least three times a week in the first 4 weeks and one to two times a week for the next 8 weeks. ${ }^{15}$ After that, there is a maintenance phase during which SET will be given for one to two times a month. As stated in their guidelines, the physical 
therapist records several outcome measurements at 3, 6, 9 and 12 months followup. ${ }^{19}$ To investigate the study's objectives, data obtained in this standard follow-up routine will be recorded prospectively.

\section{Assessments}

This study will use diagnostic and outcome measures that are recorded as part of the usual clinical practice, supplemented by several questionnaires, all specified below. Outcomes are collected from the standardised feedback letter sent by physical therapists (see online supplementary file 1) and the patient's hospital electronic health record. Table 1 provides an overview of all study assessments.

Table 1. Study timeline and assessments

\begin{tabular}{|c|c|c|c|c|c|c|}
\hline & $\begin{array}{c}\text { Baseline } \\
T_{0}\end{array}$ & $\begin{array}{c}3 m o \\
t_{1}\end{array}$ & $\begin{array}{c}6 m o \\
t_{2}\end{array}$ & $\begin{array}{c}12 \mathrm{mo} \\
t_{3}\end{array}$ & $\begin{array}{c}2 y \\
t_{4}\end{array}$ & $\begin{array}{l}5 y \\
t_{5}\end{array}$ \\
\hline \multicolumn{7}{|l|}{ Baseline characteristics } \\
\hline Sociodemographic data & $x$ & & & & & \\
\hline Cardiovascular risk factors & $x$ & & & & & \\
\hline Comorbidity & $x$ & & & & & \\
\hline Medical History & $x$ & & & & & \\
\hline Prior vascular interventions & $x$ & & & & & \\
\hline Vascular laboratory assessment & $x$ & & & & & \\
\hline Vascular imaging (DUS/CTA/MRA) & $x^{*}$ & $X^{*}$ & & & & \\
\hline \multicolumn{7}{|l|}{ Outcome measures } \\
\hline Treadmill test $\mathrm{f}$ & $x$ & $x$ & $x$ & $x$ & & \\
\hline 6MWT & $x$ & $x$ & $x$ & $x$ & & \\
\hline WHOQOL-BREF & $x$ & $x$ & $x$ & $x$ & & \\
\hline Barrier self-efficacy scale & $x$ & $x$ & $x$ & $x$ & & \\
\hline Vascuqol-6 & $x$ & $x$ & $x$ & $x$ & $x$ & $x$ \\
\hline Smoking status & $x$ & $x$ & $x$ & $x$ & $x$ & $x$ \\
\hline Attainment of treatment goal & & $x$ & $x$ & $x$ & $x$ & $x$ \\
\hline Freedom from vascular intervention & & $x$ & $x$ & $x$ & $x$ & $x$ \\
\hline Major adverse cardiovascular events & & $x$ & $x$ & $x$ & $x$ & $x$ \\
\hline \multicolumn{7}{|l|}{ Personality traits } \\
\hline Big Five Inventory & $x$ & & & & & \\
\hline HADS & $x$ & & & & & \\
\hline Brief Self-Control Score & $x$ & & & & & \\
\hline Life Orientation Test-Revised & $x$ & & & & & \\
\hline Barrier Self-Efficacy Scale & $x$ & $x$ & $x$ & $x$ & & \\
\hline
\end{tabular}

* Imaging is eligible when performed $\leq 3$ months before or after inclusion. TThe treadmill test and 6MWT are performed on different days, thus two visits are necessary per time point to collect all outcome measures. 6MWT, 6min walk test; CTA, CT angiography; DUS, duplex ultrasound scanning; HADS, Hospital Anxiety and Depression Scale; IC, intermittent claudication; MRA, magnetic resonance angiography; VascuQoL-6, Vascular Quality of Life Questionnaire-6; WHOQoL-BREF, WHO Quality of Life Questionnaire-Bref. 


\section{Baseline patient characteristics}

Participants' sociodemographic data (age, sex), medical history and comorbidity (chronic obstructive pulmonary disease, lower-extremity musculoskeletal disease, diabetes mellitus, dyslipidaemia, hypertension, kidney disease, cerebrovascular disease, ischaemic heart disease, heart failure), cardiovascular risk factors (body mass index (BMI), smoking status), prior (cardio-)vascular interventions (coronary artery bypass grafting, percutaneous coronary intervention, open or endovascular abdominal aortic aneurysm repair, lower-extremity revascularisation) and prior SET will be extracted from the electronic health records of the hospital and/or physical therapist.

\section{Vascular imaging and laboratory assessment}

Resting and postexercise ankle-brachial index (ABI) determinations in both legs are performed by trained vascular technicians in all participating centres using handheld Doppler instruments, as part of the routine work-up. The $A B I$ is defined as the ratio between the highest systolic pressure of the dorsal pedal or posterior tibial artery, and the highest of the left or right brachial pressure. To determine the anatomic location and extent of atherosclerotic disease, the ELECT Registry uses the preferred vascular imaging modality of the treating vascular surgeon, performed $\leq 3$ months before or after inclusion.

In case of magnetic resonance angiography (MRA) or CT angiography (CTA), the scan is evaluated and interpreted by experienced radiologists in the participating centres as per usual care. A stenosis of $>50 \%$ on MRA or CTA is considered significant. The reported accuracy of CTA and MRA to detect significant arterial stenosis in PAD is $>90 \%$. ${ }^{1}$ Duplex ultrasound scanning (DUS) is carried out by accredited vascular technicians to determine the location, extent and severity of the atherosclerotic lesions. A lesion is considered significant if either a peak systolic velocity ratio of $\geq 2.5$, or an end-diastolic velocity of $\geq 0.6 \mathrm{~m} / \mathrm{s}$ is observed, or if an occlusion is visualised (no flow). The reported sensitivity and specificity of DUS in patients with PAD to detect significant arterial stenoses is over $80 \%$ and $90 \%$, respectively. ${ }^{1}$

To categorise all participants according to anatomic location a team of three physicians (one vascular surgeon, two PhD candidates) will independently assess the DUS reports and readings and/or MRA or CTA scans and radiologist reports. Participants will be divided into four groups:

1. Aortoiliac lesions, containing patients with significant stenoses or occlusions in the common iliac artery, external iliac artery and/or internal iliac artery.

2. Femoropopliteal lesions, containing patients with significant stenoses or occlusions in the common femoral artery, superficial femoral artery and/ or popliteal artery.

3. Multilevel disease, containing patients with significant stenoses at both the aortoiliac and femoropopliteal level. 
4. Rest group, containing patients with no significant stenoses in the aortoiliac and femoropopliteal tract. Notably, undetected infrageniculate disease distally from the area scanned with DUS may exist in this group. This category is expected to contain some $5 \%$ of patients meeting the eligibility criteria based on a retrospective exploratory analysis of a consecutive cohort of patients from the vascular surgery outpatient clinic in the initiating centre.

The same three physicians will also assign a Transatlantic Inter-Society Consensus for the Management of PAD (TASC) classification for each arterial trajectory with a significant lesion. Notably, the inter-observer agreement for rating TASC classifications is poor. ${ }^{20} 21$ Thus, disagreement is solved by discussion and consultation of a fourth observer (vascular surgeon).

\section{Walking performance}

Treadmill-measured walking distance The maximum walking distance (MWD) and functional walking distance (FWD) will be recorded for each patient at baseline, 3, 6 and 12 months follow-up, using a standardised treadmill test. The MWD is defined as the walking distance where intolerable claudication pain forces a patient to stop. An alternative term for MWD is 'absolute claudication distance'. The FWD is defined as the distance at which the patient prefers to stop walking because of pain. Notably, this is a different measure from the 'pain-free walking distance' or 'initial claudication distance' commonly used in PAD literature. The FWD was previously found to be reliable (intraclass correlation coefficient 0.959 ) and probably a better reflection of functional impairment compared with the initial claudication distance. ${ }^{22}$ Walking distances are determined by a standardised progressive treadmill test (GardnerSkinner protocol ${ }^{23}$ ) with a constant speed of $3.2 \mathrm{~km} /$ hour starting with $0 \%$ incline, increasing every 2 min by $2 \%$. The maximum incline is $10 \%$, and the maximum duration of the test is $30 \mathrm{~min}$, resulting in a maximum distance of $1600 \mathrm{~m}$. The test protocol is advised by the Dutch physical therapy guidelines ${ }^{19}$ ClaudicatioNet therapists are taught to offer verbal encouragement and coaching during testing, but this was not standardised in this study.

6-Minute walk test To assess walking performance in a setting more resembling daily life, participants will perform the 6MWT at baseline, 3, 6 and 12 months followup. Physical therapists are instructed to perform the treadmill test and 6MWT on different days. The 6MWT records the total distance an individual is able to walk over a total of 6 min on a hard, flat surface. Participants traverse back and forth along a marked walkway. They are allowed to self-pace and rest as needed, while timing continues up to $6 \mathrm{~min}$. The 6MWT has been validated, shown to be responsive to treatment, and is predictive of mortality and mobility loss in a PAD population. ${ }^{24}$ 


\section{Quality of life}

Two questionnaires are used in the ELECT Registry to investigate the effect of treatment on QoL. The VascuQoL- 6 is a valid and responsive instrument for the assessment of health-related QoL in PAD. ${ }^{25}$ It is a shortened version of the VascuQoL-25 and contains questions relating to the patient's activities, symptoms, pain and emotional and social well-being. Answers are recorded on a 4-point scale and added up, resulting in a total score between 6 and 24. Higher scores indicate better health-related QoL. The ELECT Registry uses the Dutch version of the VascuQoL-6.

The WHOQoL questionnaire provides a generic assessment of QoL.26 27 The abbreviated version of this tool (WHOQoL-BREF) is used in this registry and was previously found to be valid and reliable. ${ }^{27}$ It contains questions on four domains, each scored on a 5-point Likert scale: physical health, psychological health, social relationships and environment. Two additional questions assess the subject's overall QoL and general health. Domain scores are obtained by averaging the scores from the individual questions and subsequent rescaling to a 0-100 scale, where higher scores represent a better QoL.

\section{Success of conservative management}

Arguably, a successful conservative treatment eliminates the need for invasive therapies. Thus, success of SET is objectified by freedom from vascular interventions, defined as either PTA (with or without stent), bypass, endarterectomy or major amputation of the lower extremities at 6 months, and 1, 2 and 5 years, follow-up.

The decision to intervene is influenced by the risk-benefit ratio of the patient's lesion and general health status, as well as the preferences of the treating vascular surgeon and patient. Thus, freedom from intervention is only a partial reflection of treatment success. To better capture patient satisfaction with treatment the attainment of the main treatment goal is recorded. At the start of each SET programme each patient determines the main treatment goal in conjunction with his or her physical therapist. This is recorded in an open text field at the start of treatment, with no restrictions with regard to the domain or measure it applies to. Satisfactory achievement of this goal ('yes' or 'no') and the (im)probability of further improvement with SET ('yes' or 'no') are followed-up at 3, 6 and 12 months and indicated in the standard feedback letter to the vascular surgeon (see online supplementary file 1).

\section{Cardiovascular mortality and morbidity}

The incidence of major adverse cardiovascular events (MACE) at 1, 2 and 5 years follow-up. Events considered are atherosclerotic cardiovascular death, non-fatal myocardial infarction and non-fatal stroke (three-component MACE), recorded from hospital records. 


\section{Psychological assessments}

The five self-report questionnaires described below assess personality traits, emotional symptoms and barrier self-efficacy. Missing data, if not completely at random, will be imputed by means of multiple imputation methodology to minimise bias. $^{28}$

\section{Big Five Inventory}

The Big Five Inventory $(\mathrm{BFI})^{29}$ consists of 44 items regarding statements of characteristics associated with five personality traits, which are openness to experiences, agreeableness, extraversion, neuroticism and conscientiousness. This study will focus on the last three traits. Every item starts with "I see myself as someone who..." and items are rated on a 5-point Likert scale (1=disagree strongly, $5=$ agree strongly). The BFI has a high test-retest reliability with intraclass correlation coefficients ranging from 0.93 to $0.96 .{ }^{30} \mathrm{~A}$ validated Dutch translation ${ }^{31}$ is used in this study, with satisfactory reliability for measuring the individual traits, with Cronbach's a ranging among traits from 0.73 to 0.86 ( $\alpha>0.7$ is a sign of good reliability). The scores will be analysed as continuous and as categorical variables. Because there are no official cut-off scores available, the median scores from the current sample will be used as cut-off points to translate scores into low and high on a specific trait ('low' < median $\geq$ 'high').

\section{Hospital Anxiety and Depression Scale}

The Hospital Anxiety and Depression Scale (HADS) is a 14-item self-report screening scale which is used to indicate the possible presence of anxiety and/or depressive symptoms. ${ }^{32}$ The scale includes seven items on anxiety and seven items on depression, both with a score ranging from 0 to 21 . The total score is classified into no anxiety or depression disorder ( $\leq 7)$, possible disorder (8-10) and probable disorder $(\geq 11)$. The Dutch translation of the HADS ${ }^{33}$ used in the ELECT Registry is validated in multiple populations. The internal consistency (Cronbach's a ranged from 0.71 to 0.90 ) and the test-retest stability (Pearson's correlation coefficient=0.91) are high.

\section{Brief self-control score}

The brief self-control score ${ }^{34}$ is used to determine self-control in which a higher score is associated with higher self-control. It consists of 13 items rated on a 5-point Likert scale (1=disagree strongly, 5=agree strongly) with a maximum score of 54 . This brief scale has a good test-retest reliability with a coefficient of 0.87 . A validated Dutch translation ${ }^{35}$ with high internal consistency (Cronbach's $\alpha=0.80$ ) will be used.

\section{Life orientation test-revised}

The life orientation test-revised ${ }^{36}$ measures optimism by means of 10 items, including four 'filler' items which do not contribute to the total score. The items are 
rated on a 5-point Likert scale ( 0 =disagree strongly, $4=$ agree strongly). The total score ranges between 0 and 24 and a higher total score is associated with a higher level of optimism. This revised test has a satisfactory test-retest reliability of $0.60-$ 0.68 . The validated Dutch translation, ${ }^{37}$ with moderate-to-high internal consistency (Cronbach's a ranging from 0.58 to 0.80 ), is used.

\section{Barrier self-efficacy scale}

The barrier self-efficacy scale (BSES) ${ }^{38}$ consists of 13 items describing the possibility that one would exercise despite the presence of possible barriers, such as bad weather or lack of interest. The inventors of the BSES determined the barriers by attributive analysis of participants' arguments for discontinuing exercise programmes. Participants indicate their degree of confidence for each item on a $0 \%$ (no confidence at all) to $100 \%$ (complete confidence) scale. The mean percentage of all items comprises the total score, in which a higher score is associated with higher barrier self-efficacy. This study uses a validated Dutch translation ${ }^{39}$ in which one question ("My work schedule conflicted with my exercise session") is left out because this would not be relevant to the majority of our study population. The internal consistency of the Dutch scale is high (Cronbach's $a=0.84$ ) and has a satisfactory test-retest stability with an intraclass correlation coefficient of 0.63 .

\section{Sample size}

We hypothesise that there will be no clinically relevant difference in changes in maximum walking distance between subjects with aortoiliac and femoropopliteal disease after 6 months. Analogous to previous trials in this population, we would consider a mean difference of $150 \mathrm{~m}$ change in walking distance between groups to be clinically relevant (SD of $300 \mathrm{~m}$ ). ${ }^{4041}$ To exclude a difference in means of $150 \mathrm{~m}$ with an a of 0.01 , a power of 0.80 ( $\beta$ of 0.2 ), 96 patients are needed per arterial disease level group. The ELECT Registry primarily aims to compare patients with single-level disease (aortoiliac vs femoropopliteal). Based on a retrospective analysis of consecutive patients with IC in our hospital, this will be the case in some $75 \%$ of the patients. Thus, for the primary analysis the required sample size amounts to a total of 256 patients $((1 / 0.75) \times(96+96))$. Assuming a conservative drop-out rate of $20 \%, 320$ subjects are required to investigate the primary objective.

\section{Statistical analysis}

Categorical variables at baseline will be presented as numbers with percentages. Continuous variables as means \pm SD if normally distributed, or as medians with interquartile ranges in case of a skewed distribution. Participants will be divided into four groups based on anatomical characteristics of disease, as apparent on the preferential imaging modality of the participating centre (either DUS, CTA or MRA). Groups are defined as follows: aortoiliac disease, femoropopliteal disease, 
multilevel disease or rest group. The primary comparison of interest is aortoiliac versus femoropopliteal disease.

Changes in walking distances (treadmill tests, 6MWT) and QoL (VascuQoL-6, WHOQoL-BREF) will be compared between groups, after multivariate adjustment. Adjustment of these measures for possible confounding variables will be performed using a general linear model with anatomical group as the independent variable. Covariates used for this adjustment are selected using univariate analysis (inclusion criteria $\mathrm{p}<0.2$ ). A stringent significance level of 0.01 will be used to account for multiple comparisons. Freedom from revascularisation, freedom from adverse cardiovascular disease events and attainment of the main treatment goal between groups will be compared using Kaplan-Meier survival curves and log rank tests. Cox proportional hazard analysis will be performed to adjust for the above-mentioned possible confounders.

Analysis for the remainder of secondary objectives is performed on the overall population (regardless of lesion location). Multivariable logistic regression will be used to determine the impact of several patient characteristics (e.g. age, sex, extent of atherosclerotic disease, comorbidity, history of cardiovascular disease and previous lower-extremity interventions) on success of conservative management. Associations between baseline personality traits and SET-related outcomes (walking distance, 6MWT, QoL and freedom from interventions), defined as change-frombaseline scores at follow-up, will be determined. For categorised BFI traits this will be analysed with unpaired t-tests (or Mann-Whitney $U$ test in case of non-normal distribution) for continuous outcomes and $\mathrm{X}^{2}$ or Fisher's exact tests for categorical outcomes. Multiple linear regression is performed with personality traits as independent variables and the SET-related outcomes as dependent variables. A hierarchical series of three models with increasing covariate adjustment will be used. In model I, age and sex are included as covariates. In model II, secondary invasive treatment (if applicable) is added. Finally, in model III, the HADS score is added in order to correct for possible symptoms of anxiety and/or depression. To analyse the change in self-efficacy at 3,6 and 12 months follow-up, a linear mixed model will be used.

\section{Data storage and retention}

All data will be handled in accordance with local regulations and privacy laws in an anonymised dataset. Physical data will be anonymised and stored accessible only by the research team, digital data will be secured using dedicated data management software 'Research Manager' (de Research Manager, Deventer, the Netherlands). After the last participant's final follow-up moment, all data will be stored for a minimum of 15 years. 


\section{Ethics and dissemination}

This trial does not hamper routine vascular surgery or physical therapy treatment for the participants. Furthermore, it mainly records diagnostic and outcome measures that are performed as part of the usual clinical routine, supplemented by several short, non-intrusive questionnaires. Therefore, the ELECT Registry has been exempted from formal medical ethical approval by the Medical Research Ethics Committees United 'MEC-U' (reference number W17.071). Nonetheless, privacy laws require that each subject must authorise the treating physician(s), therapists and institutions to release their medical information. Each subject must therefore sign a patient informed consent form before any data can be sent to the coordinating centre. The results are intended for publication in peer-reviewed journals and for presentation to stakeholders nationally and internationally.

\section{Patients and public involvement}

Patients and public were not explicitly involved in the design or conduct of this study. All participants will be informed of the results of the ELECT Registry through post or email.

\section{DISCUSSION}

The ELECT Registry will link anatomical characteristics of atherosclerotic disease to the functional and clinical outcomes of a SET-first management strategy in IC. This prospective observational study will thus primarily offer insight in the effect of lesion location on the outcomes of SET. It will produce novel results as most previously published research on SET as primary treatment either does not specify the location of the stenosis, does not include all lesions in its study design or does not include functional outcome measures. By including a diverse range of vascular surgery centres throughout the Netherlands, the study will feature a heterogenous patient population closely resembling real-world practice.

The dataset from this study will secondarily be used to identify predictors of treatment outcome from various functional and clinical variables. Previously published research aimed to investigate the effect of various patient characteristics on outcomes of conservative management in IC. ${ }^{22-44}$ However, this is the first study to include information on extent and location of PAD in such analysis. Moreover, the ELECT Registry will include a multitude of functional and clinical outcomes at both baseline and follow-up. The results will facilitate the development of a management strategy more tailored to the individual patient with IC.

The ELECT Registry is an observational study and thus prone to several validity issues. Mainly, included patients in the different lesion location groups are expected to differ regarding several clinical characteristics. Indeed, van Zitteren et al ${ }^{45}$ and Aboyans et al ${ }^{46}$ previously found differences in smoking status, diabetes mellitus, $\mathrm{BMI}$ and $\mathrm{ABI}$ between proximal and distal disease. The influence of these potential 
confounders is addressed in the study protocol by applying multivariate regression analysis. Despite this covariate adjustment, unmeasured confounding could influence the results. For instance, intensity of exercise during SET sessions is not recorded, nor daily life physical activity levels of patients. Furthermore, DUS is a non-invasive and valid tool to assess location and extent of stenosis in PAD, but has its limitations. Namely, visualisation of the iliac vessels can be limited due to body habitus and/or bowel gas, possibly introducing bias in the study design.

Strengths and limitations of this study

- This is the first prospective, longitudinal study linking the outcomes of supervised exercise therapy in intermittent claudication to the location and extent of the underlying atherosclerotic lesions.

- Apart from clinical outcome measures such as freedom from intervention, the Effect of Disease Level on Outcomes of Supervised Exercise in Intermittent Claudication Registry includes a wide range of functional measures of walking performance and quality of life.

- Potential participants are approached in 10 vascular surgery centres across the Netherlands, ensuring a varied patient population and clinical practice reflective of standard care in the Dutch healthcare system.

- The location and extent of stenosis is determined using duplex ultrasound scanning in most participants; a non-invasive and valid tool, but prone to visualisation issues in case of bowel gas or unfavourable body habitus.

- Participants with aortoiliac disease and femoropopliteal disease are expected to differ regarding several clinical characteristics (e.g. smoking status, diabetes mellitus, body mass index and ankle-brachial index) and adjustment for these variables will be performed in the analysis, but unmeasured confounding could influence the results.

\section{CONCLUSION}

The ELECT Registry, a multicentre prospective cohort study, will produce a dataset linking the functional and clinical outcomes of SET in IC to the location and extent of the atherosclerotic lesions. The results from this real-world cohort will inform clinical practice, working towards a more tailored management of IC patients. 


\section{REFERENCES}

1. Conte MS, Pomposelli FB, Clair DG, et al. Society for Vascular Surgery practice guidelines for atherosclerotic occlusive disease of the lower extremities: management of asymptomatic disease and claudication. J Vasc Surg 2015;61:2S41.

2. Frans FA, Bipat S, Reekers JA, et al. Systematic review of exercise training or percutaneous transluminal angioplasty for intermittent claudication. Br J Surg 2012;99:16-28.

3. Murphy TP, Cutlip DE, Regensteiner JG, et al. Supervised exercise versus primary stenting for claudication resulting from aortoiliac peripheral artery disease: six-month outcomes from the claudication: exercise versus endoluminal revascularization (CLEVER) study. Circulation 2012;125:130-9.

4. Spronk S, Bosch JL, den Hoed PT, et al. Intermittent claudication: clinical effectiveness of endovascular revascularization versus supervised hospital-based exercise training-randomized controlled trial. Radiology 2009;250:586-95.

5. Fakhry F, Rouwet EV, den Hoed PT, et al. Long-term clinical effectiveness of supervised exercise therapy versus endovascular revascularization for intermittent claudication from a randomized clinical trial. $\mathrm{Br} J$ Surg 2013;100:1164-71.

6. Nordanstig J, Gelin J, Hensäter M, et al. Walking performance and healthrelated quality of life after surgical or endovascular invasive versus noninvasive treatment for intermittent claudication--a prospective randomised trial. Eur J Vasc Endovasc Surg 2011;42:220-7.
7. Mazari FA, Khan JA, Carradice D, et al. Randomized clinical trial of percutaneous transluminal angioplasty, supervised exercise and combined treatment for intermittent claudication due to femoropopliteal arterial disease. Br J Surg 2012;99:39-48.

8. Fakhry F, Fokkenrood HJ, Spronk S, et al. Endovascular revascularisation versus conservative management for intermittent claudication. Cochrane Database Syst Rev 2018;3:CD010512.

9. Gommans LN, Fokkenrood HJ, van Dalen $\mathrm{HC}$, et al. Safety of supervised exercise therapy in patients with intermittent claudication. J Vasc Surg 2015;61:512-8.

10. van den Houten MM, Lauret GJ, Fakhry $F$, et al. Cost-effectiveness of supervised exercise therapy compared with endovascular revascularization for intermittent claudication. Br J Surg 2016;103:1616-25.

11. Hageman D, Fokkenrood HJP, Essers PPM, et al. Improved adherence to a stepped-care model reduces costs of intermittent claudication treatment in The Netherlands. Eur J Vasc Endovasc Surg 2017;54:51-7.

12. Makris GC, Lattimer CR, Lavida A, et al. Availability of supervised exercise programs and the role of structured home-based exercise in peripheral arterial disease. Eur J Vasc Endovasc Surg 2012;44:569-75.

13. Gommans LN, Teijink JA. Attitudes to supervised exercise therapy. Br J Surg 2015;102:1153-5.

14. Shalhoub J, Hamish M, Davies AH. Supervised exercise for intermittent claudication - an under-utilised tool. Ann R Coll Surg Engl 2009;91:473-6. 
15. Hageman $D$, van den Houten MM, Spruijt $S$, et al. Supervised exercise therapy: it does work, but how to set up a program? J Cardiovasc Surg 2017;58:305-12.

16. van Zitteren $\mathrm{M}$, Vriens $\mathrm{PW}$, Burger $\mathrm{DH}$, et al. Determinants of invasive treatment in lower extremity peripheral arterial disease. J Vasc Surg 2014;59:400-8.

17. Frans FA, Bipat S, Reekers JA, et al. SUPERvised exercise therapy or immediate PTA for intermittent claudication in patients with an iliac artery obstruction--a multicentre randomised controlled trial; SUPER study design and rationale. Eur J Vasc Endovasc Surg 2012;43:466-71.

18. Greenhalgh RM, Belch JJ, Brown LC, et al. The adjuvant benefit of angioplasty in patients with mild to moderate intermittent claudication (MIMIC) managed by supervised exercise, smoking cessation advice and best medical therapy: results from two randomised trials for stenotic femoropopliteal and aortoiliac arterial disease. Eur J Vasc Endovasc Surg 2008;36:680-8.

19. Merry AHH, Teijink JAW, Jongert MWA, et al. KNGF-Richtlijn Symptomatisch perifeer arterieel vaatlijden [In Dutch], 2017.

20. Wu TY, Giesler G, Herscu G, et al. Agreement among observers in the assignment of transatlantic inter-society consensus classification and runoff score. J Vasc Surg 2013;58:1254-8.

21. Kukkonen $\mathrm{T}$, Korhonen $\mathrm{M}$, Halmesmäki $\mathrm{K}$, et al. Poor inter-observer agreement on the TASC II classification of femoropopliteal lesions. Eur J Vasc Endovasc Surg 2010;39:220-4.
22. Kruidenier LM, Nicolaï SP, Willigendael EM, et al. Functional claudication distance: a reliable and valid measurement to assess functional limitation in patients with intermittent claudication. BMC Cardiovasc Disord 2009;9:9:9.

23. Gardner AW, Skinner JS, Cantwell BW, et al. Progressive vs singlestage treadmill tests for evaluation of claudication. Med Sci Sports Exerc 1991;23:402:408-8.

24. McDermott MM, Guralnik JM, Criqui MH, et al. Six-minute walk is a better outcome measure than treadmill walking tests in therapeutic trials of patients with peripheral artery disease. Circulation 2014; 130:61-8.

25. Larsen ASF, Reiersen AT, Jacobsen MB, et al. Validation of the Vascular quality of life questionnaire - 6 for clinical use in patients with lower limb peripheral arterial disease. Health Qual Life Outcomes 2017;15:184.

26. Öztürk Ç, te Slaa A, Dolmans DE, et al. Quality of life in perspective to treatment of postoperative edema after peripheral bypass surgery. Ann Vasc Surg 2012;26:373-82.

27. Development of the world health organization whoqol-bref quality of life assessment. The WHOQOL Group. Psychol Med 1998;28:551-8.

28. van der Heijden GJ, Donders AR, Stijnen $T$, et al. Imputation of missing values is superior to complete case analysis and the missing-indicator method in multivariable diagnostic research: a clinical example. J Clin Epidemiol 2006;59:1102-9.

29. John OP, Naumann LP, Soto CJ. Paradigm shift to the integrative Big Five trait taxonomy: History, measurement, and conceptual issues. In: John OP, Robins RW, Pervin LA, eds. Handbook of personality: Theory and research. New York, NY: Guilford Press, 2008:114-58. 
30. Arterberry BJ, Martens MP, Cadigan JM, et al. Application of generalizability theory to the big five inventory. Pers Individ Dif 2014;69:98-103.

31. Denissen JJ, Geenen R, van Aken MA, et al. Development and validation of a Dutch translation of the Big Five Inventory (BFI). J Pers Assess 2008;90:152-7.

32. Snaith RP, Zigmond AS. The hospital anxiety and depression scale. Br Med J 1986;292:344.33. Spinhoven P, Ormel J, Sloekers PP, et al. A validation study of the Hospital Anxiety and Depression Scale (HADS) in different groups of Dutch subjects. Psychol Med 1997;27:363-70.

34. TangneyJP, Baumeister RF, Boone AL. High self-control predicts good adjustment, less pathology, better grades, and interpersonal success. J Pers 2004;72:271324.

35. Helmerhorst M, de Vries Robbé M. ZelfControle Schaal en ZelfControle Schaal Observatie. Geautoriseerde Nederlandse vertaling van de Self-Control Scale (Tangney, Baumeister, \& Boone, 2004). Utrecht: Van der Hoeven Stichting, 2011.

36. Scheier MF, Carver CS, Bridges MW. Distinguishing optimism from neuroticism (and trait anxiety, self-mastery, and self-esteem): a reevaluation of the life orientation test. J Pers Soc Psychol 1994;67:1063-78.

37. Klooster PMten, Weekers AM, Eggelmeijer $F$, et al. Optimisme en/of pessimisme: factorstructuur van de nederlandse life orientation testrevised. Psychologie en Gezondheid 2010;38:89-100.

38. McAuley E. The role of efficacy cognitions in the prediction of exercise behavior in middle-aged adults. J Behav Med 1992;15:65-88.
39. Stevens M, Dijk AB-van, de Greef MHG, et al. A dutch translation of a questionnaire assessing self-efficacy in leisure-time physical activity. J Aging Phys Act 2001;9:223-32.

40. Fakhry F, Spronk S, van der Laan L, et al. Endovascular revascularization and supervised exercise for peripheral artery disease and intermittent claudication: a randomized clinical trial. JAMA 2015;314:1936-44.

41. Nicolaï SP, TeijinkJA, Prins MH. Multicenter randomized clinical trial of supervised exercise therapy with or without feedback versus walking advice for intermittent claudication. J Vasc Surg 2010;52:348-55.

42. Gardner AW, Parker DE, Montgomery PS. Predictors of improved walking after a supervised walking exercise program in men and women with peripheral artery disease. Int J Vasc Med 2016;2016:1-8.

43. Kruidenier LM, Nicolaï SP, Ten Bosch JA, et al. Predictors of walking distance after supervised exercise therapy in patients with intermittent claudication. Eur J Vasc Endovasc Surg 2009;38:449-55.

44. Dörenkamp S, Mesters I, de Bie R, et al. Patient characteristics and comorbidities influence walking distances in symptomatic peripheral arterial disease: a large one-year physiotherapy cohort study. PLoS One 2016;11:e0146828.

45. van Zitteren $M$, Vriens $P W$, Heyligers $J M$, et al. Self-reported symptoms on questionnaires and anatomic lesions on duplex ultrasound examinations in patients with peripheral arterial disease. J Vasc Surg 2012;55:1025-34.

46. Aboyans V, Lacroix P, Criqui MH. Large and small vessels atherosclerosis: similarities and differences. Prog Cardiovasc Dis 2007;50:112-25. 


\section{SUPPLEMENTARY FILE 1}

\section{Standard ClaudicatioNet Feedback Letter}

Schematic representation of components used in the ELECT Registry

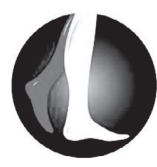

\section{Claudicatio Net}

looptherapie \& leefstijlbegeleiding

Dear [vascular surgeon / general practitioner],

[Patient name and date of birth] is currently under treatment in our therapy practice due to intermittent claudication. The main treatment goal mentioned below is the starting point for the supervised exercise therapy (SET) with lifestyle guidance.

[insert main treatment goal in open text space with a maximum of 50 words]

\section{Treatment results}

\begin{tabular}{|c|c|c|c|c|c|c|}
\hline & Date & No. of sessions & $\begin{array}{l}\text { Treadmil } \\
\text { Functiona }\end{array}$ & $\begin{array}{r}\text { rdner Sk } \\
\text { Maximal }\end{array}$ & Set speed & Motivation* \\
\hline \multicolumn{7}{|l|}{ Start } \\
\hline \multicolumn{7}{|l|}{3 months } \\
\hline \multicolumn{7}{|l|}{6 months } \\
\hline \multicolumn{7}{|l|}{9 months } \\
\hline 12 months & & & & & & \\
\hline
\end{tabular}

*Choose from: good, sufficient, bad.

Additional remarks

[open text space for additional remarks with a maximum of 50 words]

\section{Lifestyle guidance}

Part of the SET programme is modification of present risk factors for cardiovascular disease and monitoring of medication adherence.

[multiple choice questions regarding smoking status and willingness to quit, adherence to statin and/or antiplatelet drugs, willingness to attempt professional guidance on weight reduction and nutrition]

Additional remarks

[open text space for additional remarks with a maximum of 50 words] 


\section{Impeding factors}

Comorbidity with an important effect on walking and trainability:

[insert comorbity if present]

Other factors that hamper SET with lifestyle guidance:

[insert other factors if present]

Additional remarks

[open text space for additional remarks with a maximum of 50 words]

\section{Conclusion:}

Main treatment goal achieved?

Possibility of further improvement?

[yes / no / doubtful]

[yes / no / doubtful]

Follow-up advice:

[continue SET with lifestyle guidance / stop SET with lifestyle guidance]

Please heed the following factors regarding cardiovascular risk management / comorbidity / other factors hampering treatment :

[Automatically generated based on entries in the appropriate categories]

Additional remarks

[open text space for additional remarks with a maximum of 50 words]

Kind regards,

[Physical therapist name and contact information] 
protocol for a prospective, longitudinal cohort study: the ELECT Registry 



\title{
Chapter \\ The effect of arterial disease level on outcomes of supervised exercise therapy for intermittent claudication: a prospective cohort study
}

\author{
van den Houten MML \\ Jansen SCP \\ van der Laan L \\ Vriens PWHE \\ Willigendael EM \\ Koelemay MJW \\ Scheltinga MRM \\ Teijink JAW \\ ELECT Study Group.
}

Ann Surg. 2020 Jul 24. Online ahead of print 


\section{ABSTRACT}

\section{Objective}

To assess whether level of arterial obstruction determines the effectiveness of SET in patients with IC.

\section{Background Data}

Guidelines advocate SET before invasive treatment for IC, but early revascularization remains widespread, especially in patients with aortoiliac disease.

\section{Methods}

Patients were recruited from 10 Dutch centres between October 2017 and October 2018. Participants received SET first, followed by endovascular or open revascularization in case of insufficient effect. They were grouped according to level of stenosis (aortoiliac, femoropopliteal, multilevel, or rest group with no significant stenosis). Changes from baseline walking performance (maximal and functional walking distance on a treadmill test, 6 - minute walk test) and vascular quality of life questionnaire- 6 at 3 and 6 months were compared, after multivariate adjustment for possible confounders. Freedom from revascularization was estimated with Kaplan-Meier analysis.

\section{Results}

Some 267 patients were eligible for analysis (aortoiliac $n=70,26 \%$; femoropopliteal $n=115,43 \%$; multilevel $n=69,26 \%$; rest $n=13,5 \%)$. No between group differences in walking performance or vascular quality of life questionnaire- 6 were found. Mean improvement in maximal walking distance after 6 months was $439 \mathrm{~m}$ [99\% confidence interval (CI) 297-581], 466 m (99\% Cl 359-574), 353 m (99\% Cl 210-496), and $403 \mathrm{~m}(99 \% \mathrm{Cl} 58-749)$, respectively $(\mathrm{P}=0.40)$. Freedom from intervention was 73.9\% for aortoiliac disease and $88.6 \%$ for femoropopliteal disease (hazard ratio $2.46,99 \% \mathrm{Cl} 0.96-6.30, \mathrm{P}=0.013)$.

\section{Conclusions}

Short-term effectiveness of SET for IC is not determined by the location of stenosis. Although aortoiliac disease patients improved walking performance and healthrelated quality of life similarly compared to other arterial disease level groups, they underwent revascularization more often. 


\section{INTRODUCTION}

Patients with intermittent claudication (IC) due to peripheral arterial disease (PAD) are first treated with supervised exercise therapy (SET). Invasive open or endovascular revascularization (OR, ER) is considered if SET fails to satisfactorily relieve symptoms. ${ }^{1}$ With this approach the majority of patients do not require any intervention at all, even after 7 years of follow-up. ${ }^{23}$ Revascularization as initial treatment, thus unnecessary in most, leads to higher costs, ${ }^{4}$ considerable re-intervention rates, ${ }^{5}$ and more amputations. ${ }^{6}$ ER of aortoiliac stenoses is associated with more favourable procedural results and patency rates compared to revascularization in more distal disease. ${ }^{1}$ Nonetheless, SET remains the guidelineadvocated treatment of choice in all IC patients. Three randomized controlled trials compared SET with ER for aortoiliac stenoses and reported no important differences regarding walking performance or health-related quality of life. ${ }^{7-9}$ Even so, IC patients with aortoiliac disease are 4 times more often referred for early revascularization. ${ }^{10}$ This discrepancy is probably related to optimal safety and efficacy of ER in aortoiliac disease compared to femoropopliteal disease, but may also suggest that some vascular surgeons consider these patients less responsive to SET. Proximal and distal PAD indeed are different entities, associated with distinct risk factor profiles ${ }^{11,12}$ and general prognosis. ${ }^{13,14}$ Nevertheless, the effect of arterial disease level on outcomes of SET is unknown. The current study compared the effectiveness of SET in patients with IC according to the location of stenosis (aortoiliac, femoropopliteal, or multilevel disease) regarding walking performance, health-related quality of life, and clinical outcome.

\section{METHODS}

\section{Study design}

The ELECT registry is a multi-centre prospective observational study ("Netherlands Trial Register" registration number: NTR732). Participants were included between October 2017 and October 2018 in 10 vascular surgery centres across the Netherlands. A detailed account of the study methods is found in a previously published study protocol..$^{15}$ In summary, all patients diagnosed with IC (PAD Fontaine II/Rutherford 1-3) who were considered candidates for SET as primary treatment by their physicians were eligible. Patients were excluded in case of advanced stage of PAD (ischemic rest pain and/or ulcers: Fontaine $>$ II, Rutherford 4-6), vascular intervention as primary treatment, prior PAD treatment (SET or revascularization) $<12$ months before inclusion, or co-morbidity limiting proper ambulation.

This study used diagnostic and outcome measures that were recorded as part of the standard care, supplemented by a set of questionnaires and imaging of the aortoiliac and femoropopliteal tract [colour duplex ultrasound scanning (DUS), magnetic resonance angiography (MRA), or computed tomography angiography 
(CTA)]. Functional outcomes regarding walking performance and health-related quality of life were collected by the physical therapist responsible for SET and were extracted from the standardized feedback letter that is sent to the referring vascular surgeon. The participant's hospital electronic health record was used to document baseline characteristics, vascular laboratory, and imaging data. The ELECT registry was exempted from formal medical ethical approval by the Medical Research Ethics Committees United (reference number W17.071). All participants provided formal written informed consent.

\section{Treatment of subjects}

All participants were treated according to current guideline recommendations. ${ }^{1}$ In short, they received a standard regimen of SET, which entails treadmill-based or track-based exercise and lifestyle coaching. SET was provided by qualified physical therapists participating in the nationwide network ClaudicatioNet ${ }^{16}$ and following usual practice as specified in the physical therapy guidelines. ${ }^{17}$ After 3 to 6 months, a follow-up evaluation by the vascular surgeon was scheduled. During these visits, the decision to either continue conservative management or treat invasively (ER or OR) was made in a shared decision-making environment.

\section{Determination of arterial disease level}

The choice of vascular imaging modality was left to the discretion of the treating vascular surgeon. Execution of imaging, mandatorily as part of the ELECT Registry, occurred 3 months before or after inclusion. MRA and CTA were interpreted by experienced radiologists in the participating centres, as per usual care. $A>50 \%$ stenosis on MRA or CTA was considered significant. In some centres DUS, was performed, by accredited vascular technicians. A lesion was considered significant if either a peak systolic velocity ratio of $\geq 2.5$ or an end diastolic velocity of $\geq 0.6 \mathrm{~m} / \mathrm{s}$ was observed, or if flow was absent (occlusion).

A team of physicians including 1 vascular surgeon (JT) and 2 MDs (PhD candidates; $\mathrm{MH}, \mathrm{S}$ ) independently assessed all DUS and MRA or CTA scans readings and radiologist reports. Based on these assessments, participants were divided into 4 groups:

1. Aortoiliac disease: significant stenoses or occlusions in the common iliac artery, external iliac artery, and/or internal iliac artery.

2. Femoropopliteal disease: significant stenoses or occlusions in the common femoral artery, superficial femoral artery, and/or popliteal artery.

3. Multilevel disease: significant stenoses at both the aortoiliac and femoropopliteal level.

4. No disease: no significant stenoses in the aortoiliac and femoropopliteal tract. Notably, this does not rule out undetected infrageniculate disease distally from the area scanned with DUS. 
Each significant lesion was classified according to TransAtlantic Inter-Society Consensus (TASC). ${ }^{18}$ It must be appreciated that the inter-observer agreement of this classification is poor. ${ }^{19,20}$ If required, disagreement was solved by discussion and consultation of a fourth observer (vascular surgeon, MS).

\section{Study end points}

The primary objective was to compare outcomes of SET in patients with aortoiliac disease compared with femoropopliteal disease with respect to change in maximal and functional walking distance (MWD, FWD) on a standardized treadmill test. The standardized Gardner Skinner protocol, ${ }^{21}$ set at a walking speed of $3.2 \mathrm{~km} / \mathrm{h}$, was advocated in the study protocol. Nevertheless, a small portion of physical therapists deviated from this suggested protocol and adjusted the speed to the comfort of the patient (either $2 \mathrm{~km} / \mathrm{u}$ or $4.2 \mathrm{~km} / \mathrm{u}$ ). As no significant difference in set speed was identified between groups (Supplemental Table 1, Supplemental Content, http://links.Iww.com/SLA/C255), this factor was not considered in the primary analysis.

Secondary endpoints were changes in 6-minute walk test (6MWT) performance and vascular quality of life questionnaire-6 (VascuQoL-6), freedom from vascular interventions for the lower extremities, and achievement of the main treatment goal as drafted by the physical therapist and patient at the start of the SET program. All outcomes were also compared for patients with aortoiliac disease, femoropopliteal disease, multilevel disease, and the no disease group.

\section{Sample size}

We hypothesized that there is no clinically relevant difference in change in MWD between subjects with aortoiliac and femoropopliteal disease after 6 months. In an equivalence study design, to exclude a mean difference between groups of $>150 \mathrm{~m}$ change with a standard deviation (SD) of $300 \mathrm{~m}$, an a of 0.01 , and a power of $80 \%$, enrolment of 96 patients per arterial disease level group was projected.

\section{Statistical analysis}

Statistical analysis was performed with SPSS version 22 (IBM Corporation, Armonk, NY). Categorical variables were presented as numbers with percentages and compared using $\mathrm{X}^{2}$ or Fisher exact test. Continuous variables were reported as means \pm SD or as medians with interquartile range. They were compared using 1- way analysis of variance or Kruskall-Wallis rank sum tests for the 4 groups, and Tukey Honest Significant Difference test or Man-Whitney $U$ test for the comparison between aortoiliac and femoropopliteal disease, as appropriate. To account for multiple comparisons, only the 2 a priori formulated comparisons were conducted throughout the study (between the 4 groups overall and between aortoiliac and femoropopliteal disease specifically). Furthermore, a strict significance level of 0.01 was used. Missing continuous outcome and predictor data were imputed using multivariate imputation by chained equation. 
Changes from baseline walking performance (FWD, MWD, 6MWT) and Vascuqol-6 sum scores at 3-and 6-months follow-up were compared between groups after multivariate adjustment. To this end, a general linear model was used with disease level as the independent variable. Covariates used for this adjustment were selected in univariable and multivariable methods. Effects with a P-value of less than 0.2 were considered significant. First, baseline variables displaying a significant difference between aortoiliac and femoropopliteal groups were entered in the multivariable model. Then, covariates were selected using backwards elimination in the multivariable analysis to keep only factors significantly affecting change in MWD in the model. Sex, age, and body-mass index were included regardless of P-value, as the literature considers these parameters as predictors of walking performance. ${ }^{22-24}$ Walking performance data are generally nonnormally distributed, thus for the general linear model the assumption of normality of the residuals was confirmed. A detailed account on the effect of various baseline measures on the different outcome measures in the ELECT Registry will be published separately.

Freedom from intervention between groups was estimated using KaplanMeier survival analysis and compared with log rank tests. Cox proportional hazard analysis was used to correct for the effect of several unevenly distributed potential confounders $(P<0.05$, and $P<0.2)$ between disease level groups at baseline. The time to attainment of the main treatment goal was not exactly measured, but rather determined at fixed intervals ( 3 and 6 months). Thus, instead of the preplanned Kaplan-Meier survival analysis for this outcome, rates for attainment of the treatment goal were compared between groups using $X^{2}$ at 3 months and 6 months follow-up.

\section{Sensitivity analysis}

Several sensitivity analyses assessed the impact of methodological decisions on the conclusions. First, we conducted an analysis without imputation (complete-case analysis). Second, we performed a "per protocol" analysis were all patients who underwent an intervention were excluded. Third, a supplemental analysis redefining disease level to "inflow" lesions (aortoiliac and multilevel disease) versus "outflow" lesions (no evidence of aortoiliac disease).

\section{RESULTS}

During the 1-year inclusion period, 493 patients were evaluated and 343 patients were willing to participate in the ELECT registry. As 46 were excluded for reasons listed in Figure 1, a total of 297 patients participated in the study. Data were missing or incomplete in 30; therefore, 267 patients were eligible for the primary analysis (aortoiliac disease $n=70,26 \%$; femoropopliteal disease $n=115,43 \%$; multilevel disease $n=69,26 \%$; no significant stenosis in either tract $n=13,5 \%$ ). 


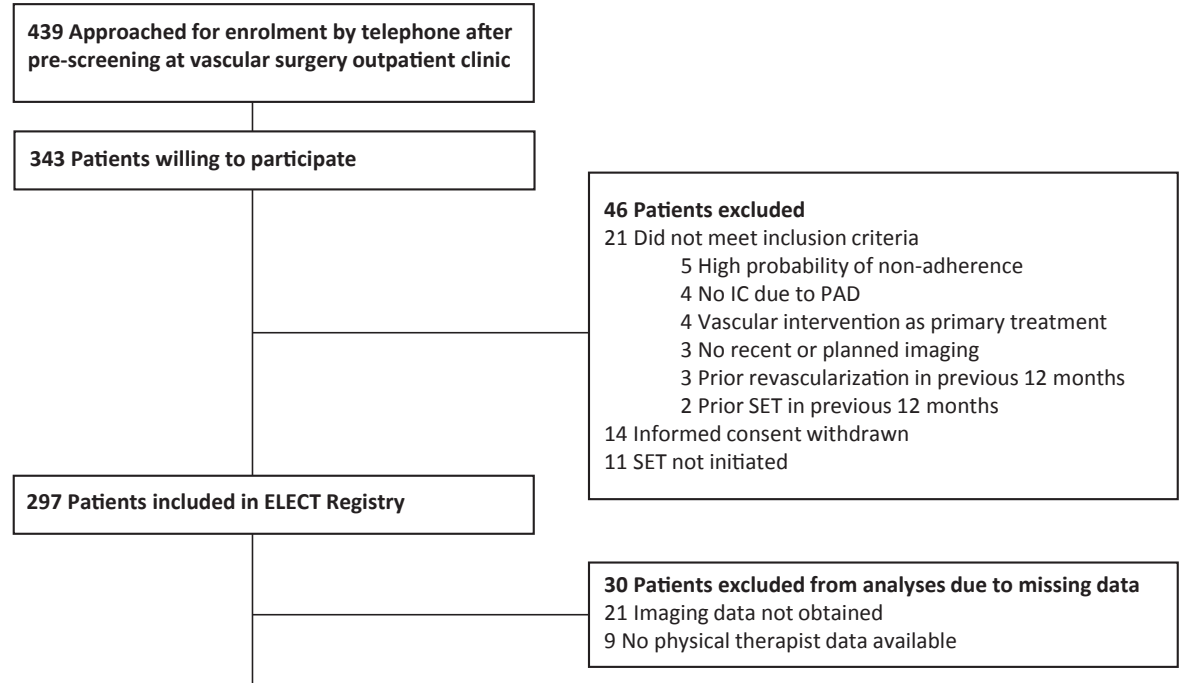

267 Patients included in primary analysis

Figure 1 - Flow chart of the inclusion process

Baseline characteristics per group are compared in Table 1. In general, participants with aortoiliac disease were on average 7 years younger, had diabetes mellitus over 3 times less often, and had higher ankle brachial index ( $\mathrm{ABI}$ ) values and less severe TASC scores compared to participants with femoropopliteal disease. Participants had completed a mean number of $17 \pm 5(n=204)$ SET sessions after 3 months, and $26 \pm 6(n=171)$ after 6 months. The mean number of SET sessions were not different between the aortoiliac, femoropopliteal, multilevel, and no disease group at 3 months $(17 \pm 5,17 \pm 5,20 \pm 4,17 \pm 5$, respectively; $P=0.27)$ and 6 months followup $(26 \pm 6,26 \pm 6,26 \pm 7,29 \pm 6$, respectively; $P=0.64)$.

\section{Walking performance and health-related quality of life}

Unadjusted changes from baseline after 3 and 6 months of SET are shown in Table 2. Patients with aortoiliac, femoropopliteal, or multilevel stenoses all showed significant improvements in MWD, FWD, 6MWD, and Vascuqol-6 sum scores. Participants in the "no disease group" did not improve in Vascuqol-6 and 6MWT. No statistically significant differences in outcomes between overall disease level groups and between patients with femoropopliteal and aortoiliac disease were found. 


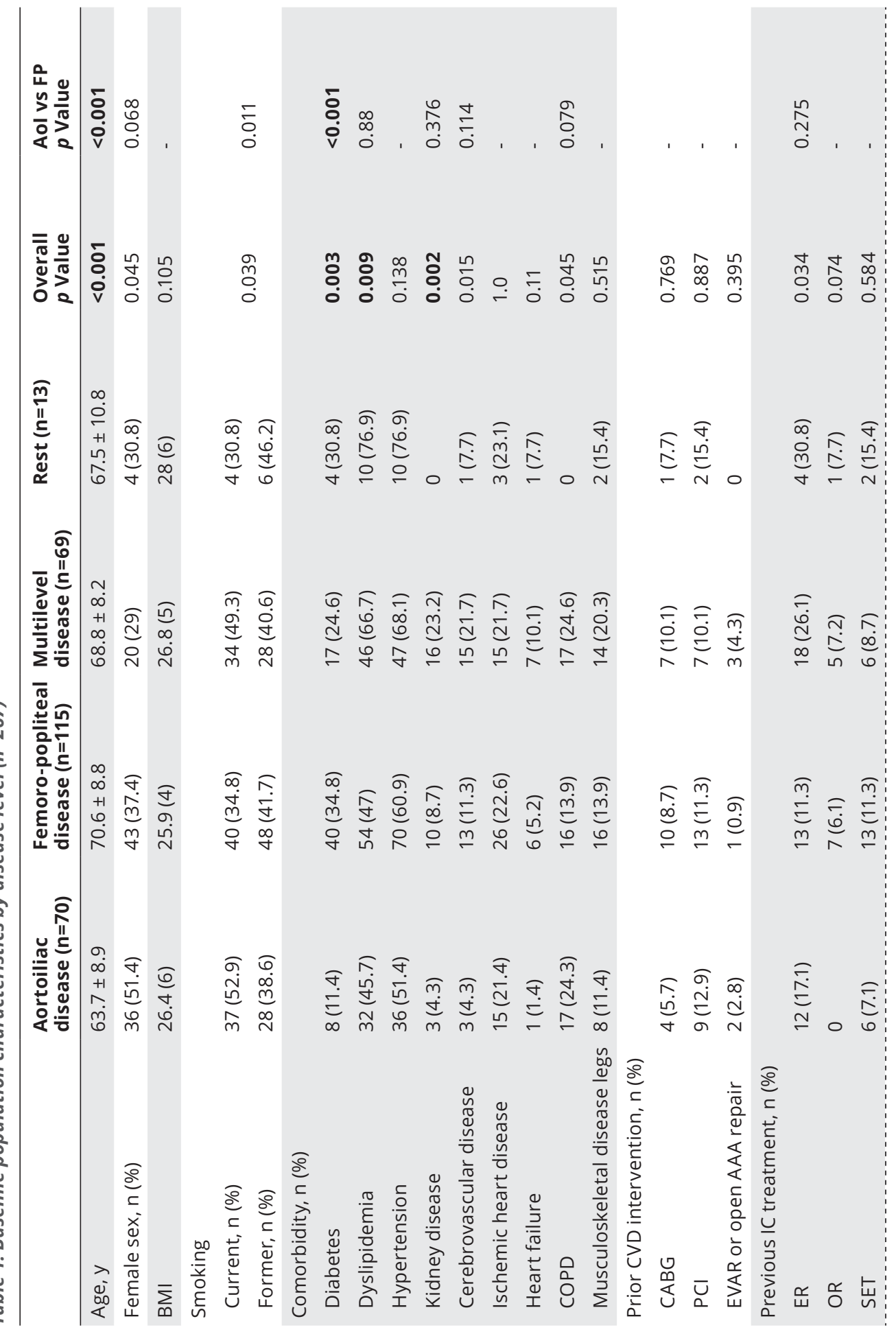




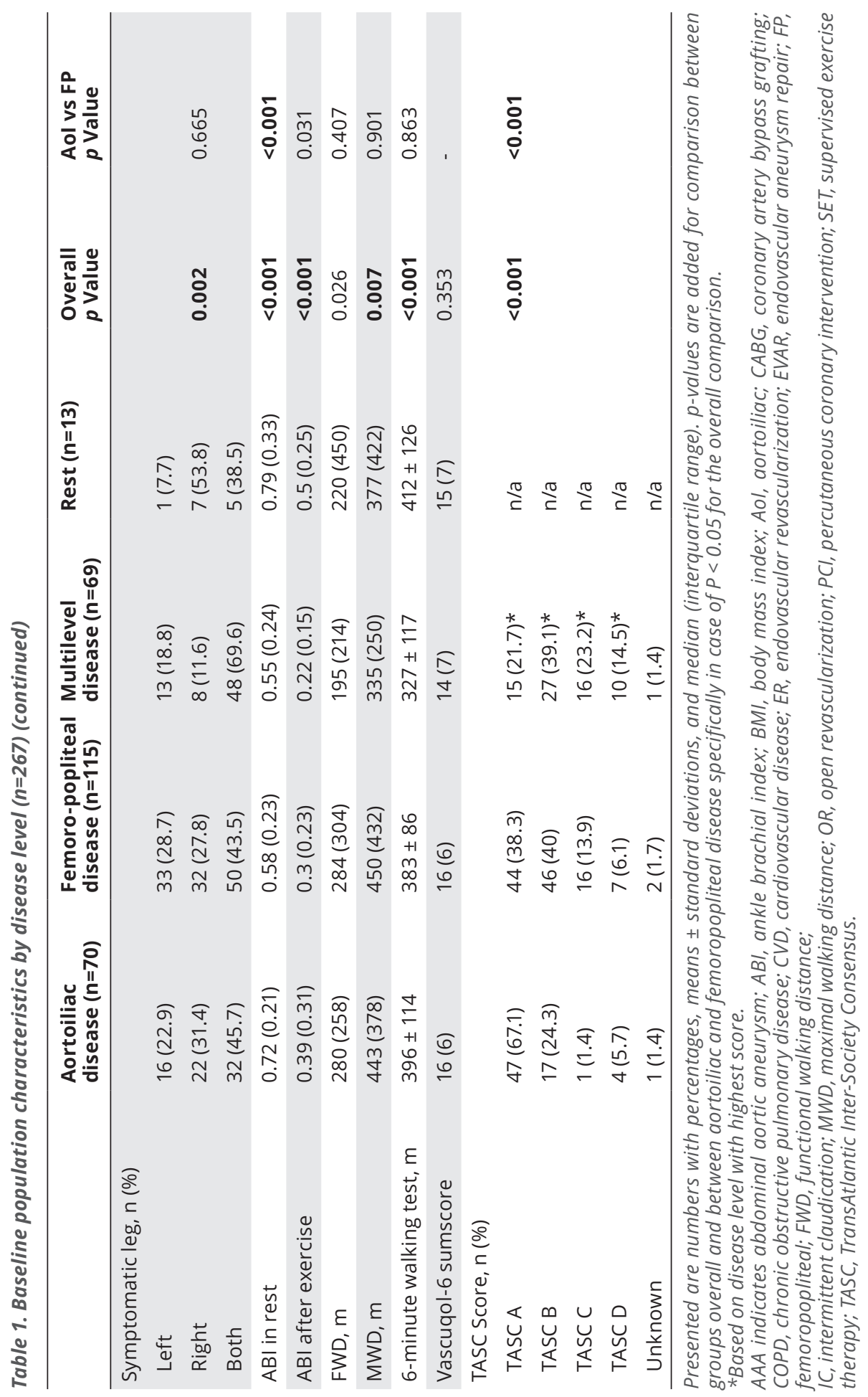




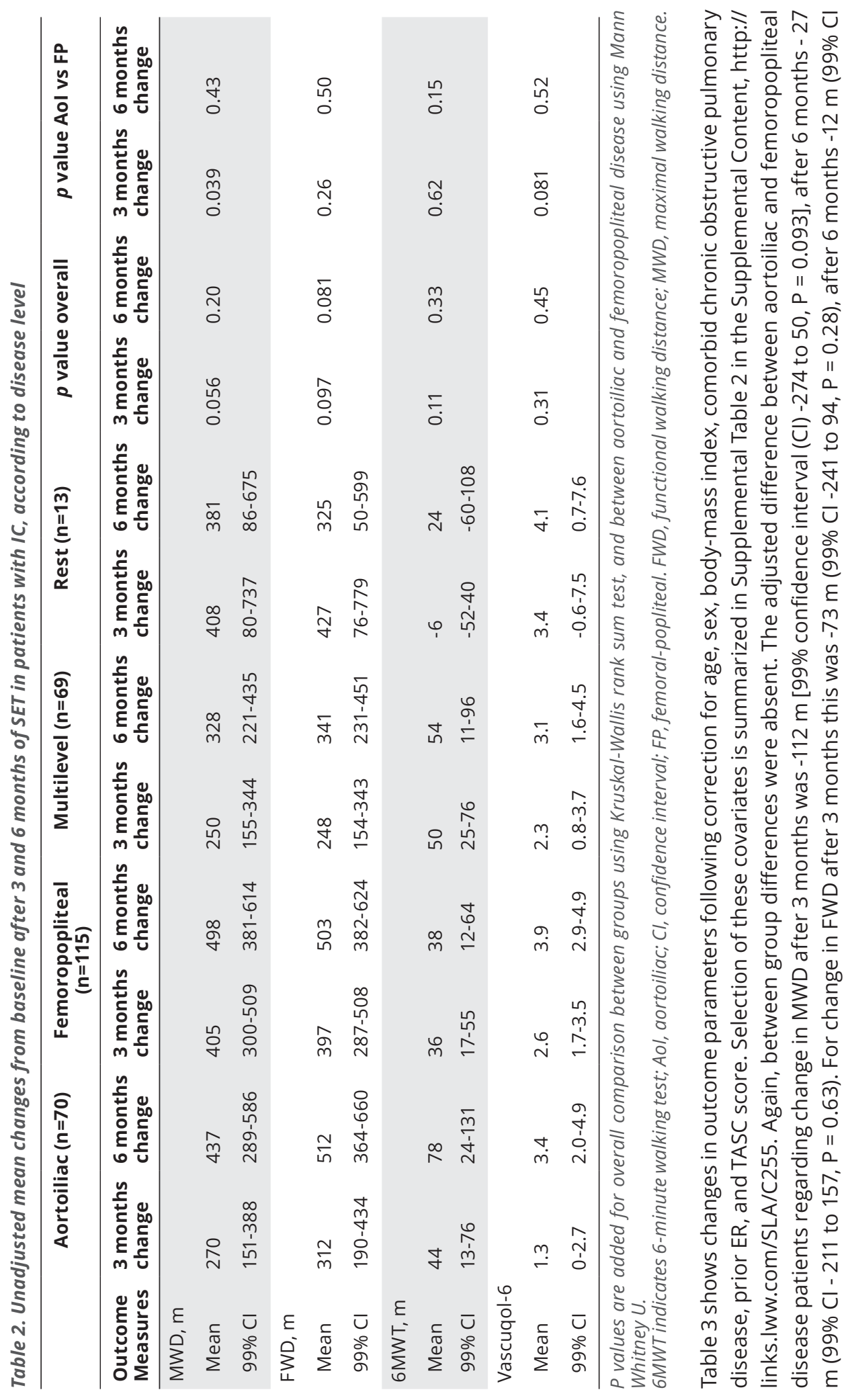




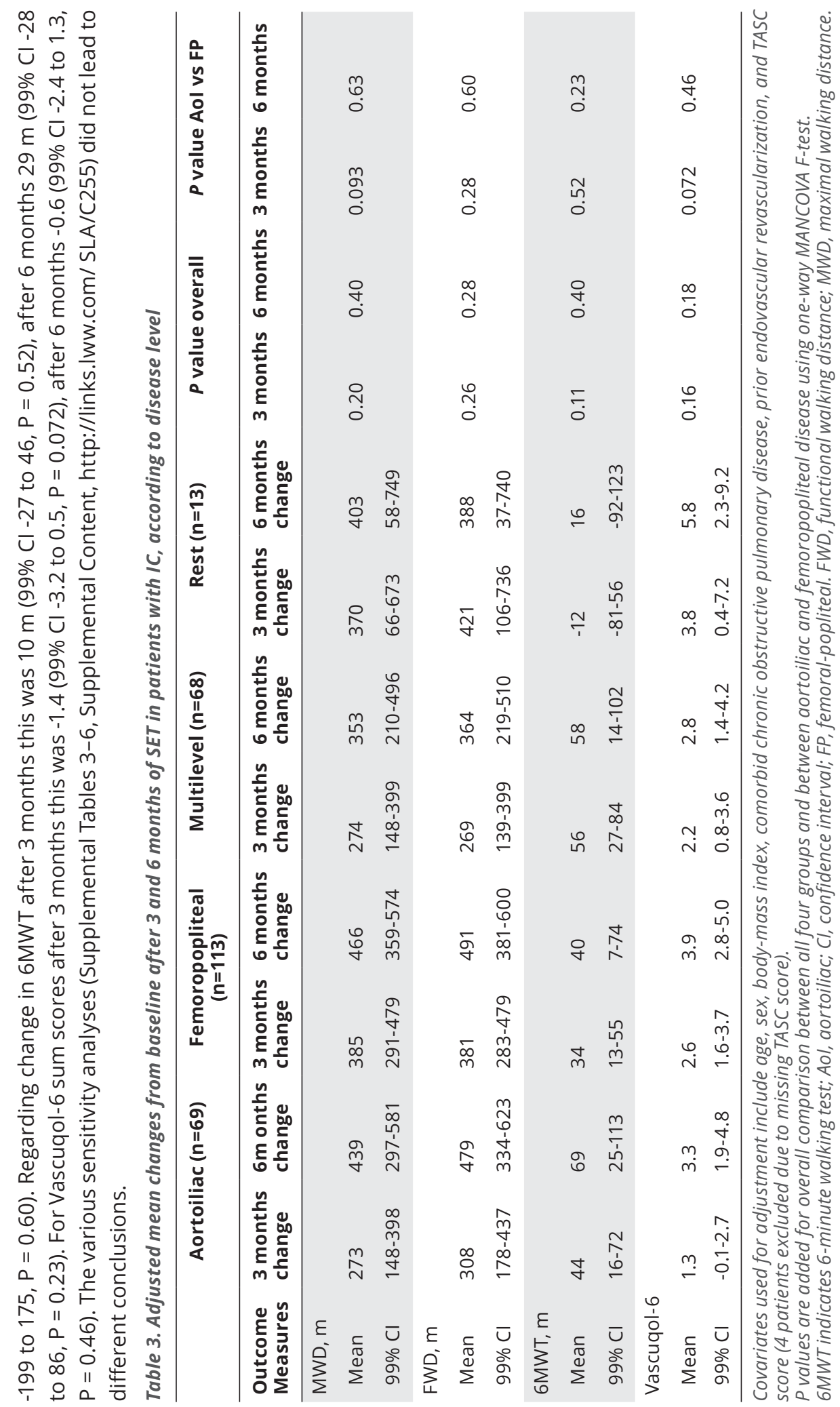




\section{Freedom from revascularization}

After 6 months follow-up, 73.9\% (51/69) of patients in the aortoiliac group remained free from intervention, compared to $88.6 \%$ (101/114) of femoropopliteal disease patients, $75.4 \%$ (52/69) of multilevel disease patients and all (100\%) participants in the no disease group. Kaplan-Meier survival curves (Figure 2) show that betweengroup differences start to seem after 3 months of follow-up (log rank test for overall comparison: $\mathrm{X}^{2}=10.92, \mathrm{P}=0.012$; for aortoiliac versus femoropopliteal disease $\left.X^{2}=6.559, P=0.0104\right)$. Subsequently, the association between aortoiliac disease versus femoropopliteal disease and freedom from revascularization was assessed while adjusting for potential confounding variables that were distributed differently between disease level groups at baseline, using Cox proportional hazard analysis (Table 4). Aortoiliac disease was associated with a statistically significant higher risk for early revascularization in the adjusted, but not the unadjusted, analysis. For the overall population, only resting $A B I$ was identified as additional significant predictor of early revascularization (Supplemental Table 7, Supplemental Content, http://links.Iww.com/SLA/C255).

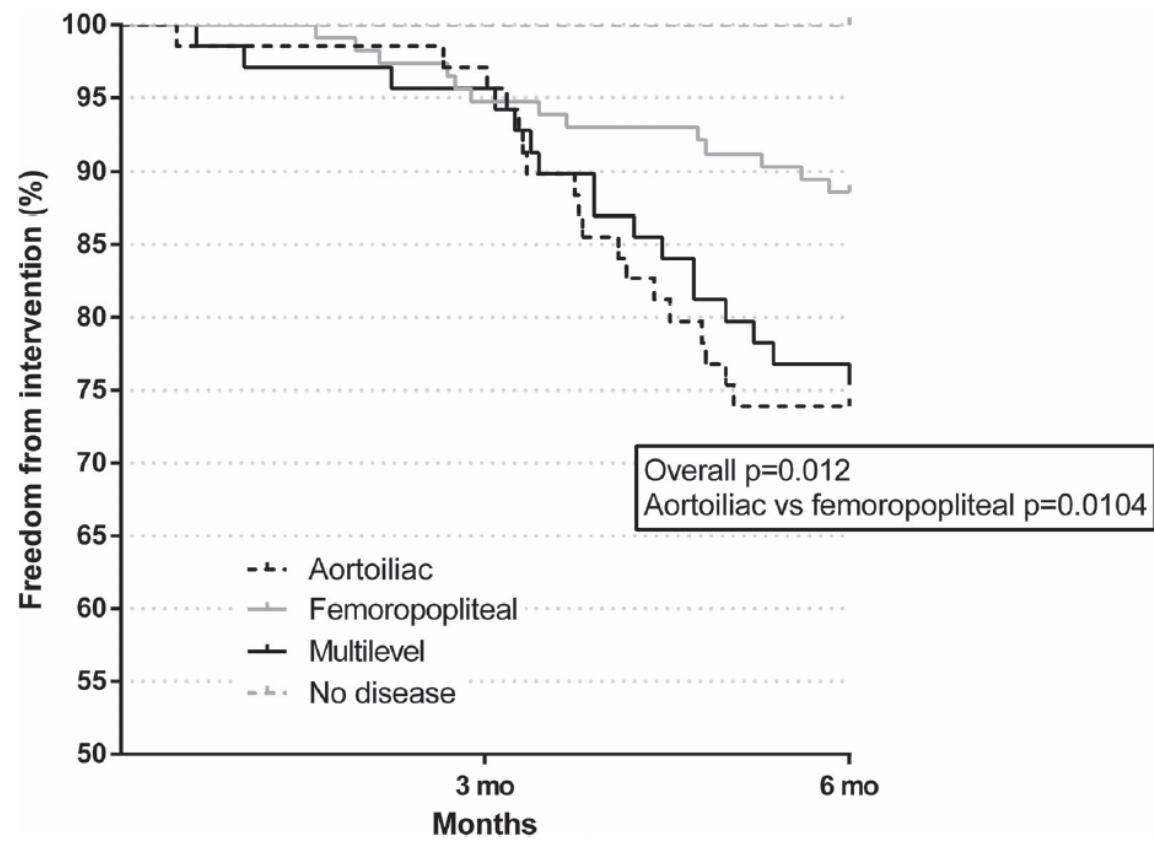

Figure 2 - Freedom from intervention after 6 months of treatment 
Table 4. Cox proportional hazard analysis of association of aortoiliac disease versus femoropopliteal disease with need for revascularization after 6 months follow-up

\begin{tabular}{lll}
\hline & Hazard ratio $(\mathbf{9 9 \%} \mathrm{Cl})$ & p value \\
\hline Unadjusted & $2.46(0.96-6.30)$ & 0.013 \\
Model 1 (adjusted)* & $2.99(1.09-8.05)$ & 0.005 \\
Model 2 (adjusted)* & $3.82(1.11-13.11)$ & 0.005 \\
Model 3 (adjusted)* & $3.68(1.04-13.05)$ & 0.008 \\
\hline
\end{tabular}

* Models adjusted for: Model 1 = TASC score (5 patients excluded because of missing data); Model $2=$ Model $1+$ age, smoking status, diabetes, ankle brachial index in rest and after exercise; Model $3=$ Model $2+$ sex, cerebrovascular accident, and chronic obstructive pulmonary disease.

$\mathrm{Cl}$ indicates confidence interval; $H R$, hazard ratio.

\section{Attainment of the treatment goal}

Data accrual for attainment of the main treatment goal was poor with $87 / 267$ (32.6\%) missing cases at 3 months, and 111/267 (41.6\%) at 6 months. Nonetheless, of the participants with complete data sets, 7/46 (15.9\%) patients with aortoiliac disease, $25 / 79$ (31.6\%) femoropopliteal disease patients, $11 / 45$ (24.4\%) of the multilevel group, and $1 / 10(10 \%)$ of the no disease group had attained their treatment goal at the 3 months follow-up visit $(P=0.14)$. After 6 months of therapy these percentages were $20 / 40$ (50\%), 33/70 (47.1\%), 15/38 (39.5\%), and $3 / 8(37.5 \%)$, respectively ( $P=0.79)$.

\section{DISCUSSION}

This prospective observational study demonstrates that patients with IC achieve equal benefits after 3 and 6 months of SET, regardless of arterial disease location. Disease level groups showed similar improvements in walking performance and health-related quality of life, and rates of attainment of the treatment goal. Nonetheless, patients with aortoiliac disease appeared more likely to undergo a vascular intervention compared to femoropopliteal disease, especially after adjustment for baseline differences.

The results of the ELECT registry justify guideline recommendations advocating exercise therapy first, before considering more invasive treatment options. IC patients with aortoiliac, femoropopliteal, and multilevel stenoses showed meaningful improvements on all outcomes beyond previously established minimally important differences. ${ }^{25-27}$ No between-group differences were present. These results are consistent with previous randomized trials on the effectiveness of $\mathrm{SET}, 2,28$ and the presumed working mechanisms of exercise therapy in PAD. With $\mathrm{SET}$, improvement of claudication symptoms is established due to a combination of (cardiovascular) systemic mechanisms and adaptations in pain tolerance, rather than improving measures of limb vascular resistance such as the ABI. ${ }^{29}$ Moreover, alternative modes of exercise such as upper-extremity training seem to have similar effects on walking performance compared with walking exercise. ${ }^{30}$ This 
study confirms that the distribution of atherosclerotic disease does not determine any functional outcomes of SET. As a consequence, it is not necessary to obtain imaging (CTA, MRA, or duplex) of the lower extremity arteries before referral to a physical therapist contributing to the cost-effectiveness of SET. Although this is already recommended in current guidelines, it is not widespread standard practice. ${ }^{1}$

In this study, single-level aortoiliac disease appeared associated with a higher probability of undergoing vascular interventions. Likewise, multilevel disease patients showed similar intervention rates probably attesting to the practice of "fixing the inflow first" among vascular professionals. An earlier study identified proximal disease as the strongest predictor of primary revascularization as opposed to conservative management. ${ }^{10}$ Apart from any functional improvements after SET, the risk-benefit ratio of a possible intervention understandably plays a role in a shared decision to intervene. In aortoiliac disease, risks are less and benefits more durable, compared to ER in more distal lesions. ${ }^{1}$ Moreover, in the current study, patients with aortoiliac disease had overall less severe TASC scores and were younger than femoropopliteal disease patients, factors possibly playing a role in the trend towards more interventions. When correcting for these and other factors, statistical certainty for the difference in freedom from revascularization increased.

In general, the need for revascularization in this study cohort (23\%) was higher than reported in other Dutch population-based series with longer follow-up (6\%19\%). ${ }^{5,31}$ This may be partly explained by bias introduced by the study design and setting. First, as dictated by the inclusion criteria, the location of disease was known for all patients in the analysis. This knowledge may have lowered the threshold to intervene. Second, all participants were recruited from outpatient vascular surgery clinics. Over the past years, a growing sample of patients in the Netherlands is referred to SET by a first-line general practitioner. As only the presence of symptoms of IC and a valid ABI reading suffice for an appropriate referral, a consultation of a vascular surgeon is generally not needed unless more invasive treatment is possibly indicated. In addition, a hospital patient population may be more inclined to a vascular intervention by default. Furthermore, the proportion of patients with prior vascular interventions was relatively high, as was having TASC A lesions. Interestingly; however, only aortoiliac disease and resting $A B I$ appeared significant predictors for early revascularization. More research is needed to elucidate the determinants of the need for intervention relative to location of disease and functional outcomes. The longer-term results from the ELECT registry will be used in this regard.

The ELECT registry is the first study to couple the extent and location of atherosclerotic disease in IC patients treated with SET to a wide range of functional and clinical outcome measures. However, several limitations should be taken in consideration when interpreting the results. First, a relatively large number of patients declined participation, possibly introducing bias where only patients most motivated for the treatment are reflected in the results. Second, more participants 
were having multilevel disease than projected causing a smaller sample size in the aortoiliac group. Therefore, the current study lacked power for detecting small differences in outcomes between groups, especially with the stringent $P<0.01$ significance level. However, the sample size was sufficient to detect clinically relevant differences, especially in the inflow versus outflow disease sensitivity analysis. Moreover, the reported P-values exceed more conservative significance levels, especially after correction for confounders. Third, although a wide range of baseline characteristics were measured and accounted for in the analyses, unmeasured confounding may possibly have influenced the results. For instance, the intensity of exercise during SET sessions is not recorded, nor daily life physical activity levels. Fourth, DUS is a non-invasive and accurate tool to assess location and extent of stenosis in PAD, but has its limitations. For instance, visualization of the iliac vessels can be limited due to body habitus and/or bowel gas, possibly introducing bias in the study design. Finally, the current report shows short-term results but not any long-term data.

In conclusion, the efficacy of SET with regards to improving walking performance and health-related quality of life in IC patients in the short term is not influenced by arterial disease level. Despite equal improvements in functional measures, aortoiliac disease patients were prone to early revascularization compared with patients with femoropopliteal stenoses. This study confirms that all IC patients should receive a trial of exercise therapy before invasive treatment is considered, regardless of the location or extent of the stenosis.

\section{ACKNOWLEDGEMENTS}

The authors would like to acknowledge all 219 participating physical therapists participating in ClaudicatioNet for their help in data collection. Furthermore, we thank all outpatient clinic personnel involved in conducting the study who were not credited in the ELECT Study Group or as an author on this paper.

\section{The ELECT Study Group}

Catharina Hospital, Eindhoven: Joep AW Teijink, Marc RHM van Sambeek, Philippe WM Cuypers, Marijn ML van den Houten, Sandra CP Jansen, Anneroos Sinnige Amphia Hospital, Breda: Lijckle van der Laan, Gwan H Ho, Thijs MG Buimer Elisabeth Twee Steden Hospital, Tilburg: Patrick WHE Vriens Medical Spectrum Twente, Enschede: Edith M Willigendael VieCuri Medical Centre, Venlo: Jan-Willem M Elshof Rijnstate Hospital, Arnhem: Jan Willem P Lardenoije Maxima Medical Centre, Veldhoven: Marc RM Scheltinga Albert Schweitzer Hospital, Dordrecht: Maarten A Lijkwan University Medical Centre Utrecht, Utrecht: Eline S van Hattum Amsterdam University Medical Centres, Amsterdam: Mark JW Koelemay 


\section{REFERENCES}

1. Aboyans V, Ricco JB, Bartelink MEL, et al. Editor's choice - 2017 ESC guidelines on the diagnosis and treatment of peripheral arterial diseases, in collaboration with the european society for vascular surgery (ESVS). Eur J Vasc Endovasc Surg. 2018;55:305-368.

2. Fakhry F, Fokkenrood HJ, Spronk S, et al. Endovascular revascularisation versus conservative management for intermittent claudication. Cochrane Database Syst Rev. 2018;3:CD010512.

3. Fakhry F, Rouwet EV, den Hoed PT, et al. Long-term clinical effectiveness of supervised exercise therapy versus endovascular revascularization for intermittent claudication from a randomized clinical trial. Br J Surg. 2013; 100:1164-1171.

4. van den Houten MM, Lauret GJ, Fakhry $F$, et al. Cost-effectiveness of supervised exercise therapy compared with endovascular revascularization for intermittent claudication. Br J Surg. 2016;103:1616-1625.

5. Hageman D, Fokkenrood HJP, Essers PPM, et al. Improved adherence to a stepped-care model reduces costs of intermittent claudication treatment in the Netherlands. Eur J Vasc Endovasc Surg. 2017;54:51-57.

6. Golledge J, Moxon JV, Rowbotham S, et al. Risk of major amputation in patients with intermittent claudication undergoing early revascularization. $\mathrm{Br} J$ Surg. 2018;105:699-708.

7. Murphy TP, Cutlip DE, Regensteiner JG, et al. Supervised exercise versus primary stenting for claudication resulting from aortoiliac peripheral artery disease: six-month outcomes from the claudication: exercise versus endoluminal revascularization (CLEVER) study. Circulation. 2012;125:130-139.
8. Spronk S, Bosch JL, den Hoed PT, et al. Intermittent claudication: clinical effectiveness of endovascular revascularization versus supervised hospital-based exercise trainingrandomized controlled trial. Radiology. 2009; 250:586-595.

9. Koelemay MJ, Frans FA, Buscher $H C$, Vermeulen EG, Legemate DA, Reekers JA on behalf of the Super Study Investigators. Supervised Exercise Therapy or Immediate PTA for Intermittent Claudication in Patients With an Iliac Artery Obstruction - A Randomized Controlled Trial. Abstract Presented at '31st Annual Meeting of the European Society for Vascular Surgery (ESVS) Lyon, France, 19 September 2017

10. van Zitteren $\mathrm{M}$, Vriens PW, Burger DH, et al. Determinants of invasive treatment in lower extremity peripheral arterial disease. J Vasc Surg. 2014;59:400-408.e2.

11. Aboyans V, Lacroix P, Criqui MH. Large and small vessels atherosclerosis: similarities and differences. Prog Cardiovasc Dis. 2007; 50:112-125.

12. Aboyans V, Criqui MH, Denenberg JO, et al. Risk factors for progression of peripheral arterial disease in large and small vessels. Circulation. 2006; 113:2623-2629.

13. Chen Q, Smith CY, Bailey KR, et al. Disease location is associated with survival in patients with peripheral arterial disease. J Am Heart Assoc. 2013;2:e000304.

14. Aboyans V, Desormais I, Lacroix P, et al. The general prognosis of patients with peripheral arterial disease differs according to the disease localization. J Am Coll Cardiol. 2010;55:898-903. 
15. van den Houten MM, Jansen SC, Sinnige A, et al. Protocol for a prospective, longitudinal cohort study on the effect of arterial disease level on the outcomes of supervised exercise in intermittent claudication: the ELECT registry. BMJ Open. 2019;9:e025419.

16. Lauret GJ, Gijsbers HJ, Hendriks EJ, et al. The ClaudicatioNet concept: design of a national integrated care network providing active and healthy aging for patients with intermittent claudication. Vasc Health Risk Manag 2012;8:495- 503.

17. Merry AHH, Teijink JAW, Jongert MWA, et al. KNGF-Richtlijn Symptomatisch Perifeer Arterieel Vaatlijden [In Dutch],Available at: https:// www.kngf.nl/kennisplatform/ richtlijnen/symptomatisch-perifeerarterieelvaatlijden. Accessed February 24, 2020.

18. Steering Committee TASC, Jaff MR, White CJ, et al. An Update on Methods for Revascularization and Expansion of the TASC Lesion Classification to Include Below-the-Knee Arteries: A Supplement to the Inter-Society Consensus for the Management of Peripheral Arterial Disease (TASC II). Vasc Med. 2015;20:465478.

19. Wu TY, Giesler G, Herscu G, et al. Agreement among observers in the assignment of TransAtlantic Inter-Society Consensus classification and runoff score. J Vasc Surg. 2013;58:1254-1258.

20. Kukkonen $\mathrm{T}$, Korhonen $\mathrm{M}$, Halmesmaki $\mathrm{K}$, et al. Poor inter-observer agreement on the TASC II classification of femoropopliteal lesions. Eur J Vasc Endovasc Surg. 2010;39:220-224.

21. Gardner AW, Skinner JS, Cantwell BW, et al. Progressive vs single-stage treadmill tests for evaluation of claudication. Med Sci Sports Exerc. 1991;23:402-408.
22. Dorenkamp S, Mesters I, de Bie R, et al. Patient characteristics and comorbidities influence walking distances in symptomatic peripheral arterial disease: a large one-year physiotherapy cohort study. PLoS One. 2016;11: e0146828.

23. Kruidenier LM, Nicolai SP, Ten Bosch JA, et al. Predictors of walking distance after supervised exercise therapy in patients with intermittent claudication. Eur J Vasc Endovasc Surg. 2009;38:449-455.

24. Gardner AW, Parker DE, Montgomery PS. Predictors of improved walking after a supervised walking exercise program in men and women with peripheral artery disease. Int J Vasc Med. 2016;2016:2191350.

25. Conijn AP, Bipat S, Reekers JA, et al. Determining the minimally important difference for the VascuQol sumscore and its domains in patients with intermittent claudication. Eur J Vasc Endovasc Surg. 2016;51:550-556.

26. van den Houten MM, Gommans LN, van der Wees PJ, et al. Minimally important difference of the absolute and functional claudication distance in patients with intermittent claudication. Eur J Vasc Endovasc Surg. 2016; 51:404-409.

27. Gardner AW, Montgomery PS, Wang M. Minimal clinically important differences in treadmill, 6-minute walk, and patientbased outcomes following supervised and home-based exercise in peripheral artery disease. Vasc Med. 2018;23:349357

28. Hageman D, Fokkenrood HJ, Gommans $L N$, et al. Supervised exercise therapy versus home-based exercise therapy versus walking advice for intermittent claudication. Cochrane Database Syst Rev. 2018;4:CD005263. 
29. Hamburg NM, Balady GJ. Exercise rehabilitation in peripheral artery disease: functional impact and mechanisms of benefits. Circulation. 2011;123:87-97.

30. Lauret GJ, Fakhry F, Fokkenrood HJ, et al. Modes of exercise training for intermittent claudication. Cochrane Database Syst Rev. 2014;CD009638.

31. Fokkenrood HJ, Scheltinga MR, Koelemay MJ, et al. Significant savings with a stepped care model for treatment of patients with intermittent claudication. Eur J Vasc Endovasc Surg. 2014;48:423429. 


\section{SUPPLEMENTAL CONTENT}

\section{Treadmill speed}

i. Supplemental Table 1. Walking speed used for treadmill testing per disease level group.

\section{Selection of covariates}

ii. Supplemental Table 2. Multivariable linear regression analysis of factors independently correlated with change in maximal walking distance from baseline at 6 months.

\section{Complete case analysis}

iii. Supplemental Table 3. Unadjusted mean changes from baseline after 3 and 6 months of SET in patients with IC, according to disease level, for the complete case.

iv. Supplemental Table 4. Adjusted mean changes from baseline after 3 and 6 months of SET in patients with IC, according to disease level, for the complete case.

\section{Per-protocol analysis}

v. Supplemental Table 5. Adjusted mean changes from baseline after 3 and 6 months of SET in patients with IC, according to disease level, excluding patients who underwent early revascularization.

\section{Inflow versus outflow analysis}

vi. Supplemental Table 6. Adjusted mean changes from baseline after 3 and 6 months of SET in patients with IC with inflow disease versus outflow disease.

\section{Predictors of early revascularization}

vii. Supplemental Table 7. Multivariable analysis of predictors of early revascularization at 6-months clinical follow-up. 


\section{TREADMILL SPEED}

Supplemental Table 1. Walking speed used for treadmill testing per disease level group.

\begin{tabular}{llllll}
\hline & $\begin{array}{l}\text { Aortoiliac } \\
\text { disease } \\
(\mathbf{n = 5 9 )}\end{array}$ & $\begin{array}{l}\text { Femoral- } \\
\text { popliteal } \\
\text { disease } \\
(\mathbf{n = 8 9 )}\end{array}$ & $\begin{array}{l}\text { Multilevel } \\
\text { disease } \\
(\mathbf{n = 5 9 )}\end{array}$ & $\begin{array}{l}\text { No disease } \\
(\mathbf{n = 1 1 )}\end{array}$ & $\boldsymbol{P}$ \\
\hline Treadmill speed, no. & & & & & \\
\hline $2 \mathrm{~km} / \mathrm{h}$ & $1(1.7 \%)$ & $3(3.4 \%)$ & $7(11.9 \%)$ & $1(9.1 \%)$ & \\
\hline $3.2 \mathrm{~km} / \mathrm{h}$ & $50(84 . \%)$ & $74(83.1 \%)$ & $49(83.1 \%)$ & $9(81.8 \%)$ & 0.19 \\
\hline $4.4 \mathrm{~km} / \mathrm{h}$ & $8(13.6 \%)$ & $12(10.4 \%)$ & $4(6.8 \%)$ & $1(9.1 \%)$ & \\
\hline
\end{tabular}

$P$ values are added for overall comparison between groups using Pearson's $\chi^{2}$ 


\section{SELECTION OF COVARIATES}

Supplemental Table 2. Multivariable linear regression analysis of factors independently correlated with change in maximal walking distance from baseline at 6 months.

\begin{tabular}{|c|c|c|c|c|}
\hline \multirow[t]{2}{*}{ Variables } & \multicolumn{2}{|c|}{ Multivariable model } & \multicolumn{2}{|c|}{ Final model } \\
\hline & $P$ value & Beta per unit & $P$ value & Beta per unit \\
\hline Female sex & 0.65 & 15 & 0.61 & 21 \\
\hline Age, y & 0.24 & 4 & 0.31 & 3 \\
\hline Body mass index, $\mathrm{kg} / \mathrm{m}^{2}$ & 0.18 & -11 & 0.19 & -10 \\
\hline$A B I$ in rest & 0.45 & -149 & - & - \\
\hline $\mathrm{ABI}$ after exercise & 0.30 & -206 & - & - \\
\hline Smoking status & & & - & - \\
\hline Never & 0.47 & -63 & & \\
\hline Current smoker & 0.74 & 17 & - & - \\
\hline Former smoker & Reference & Reference & - & - \\
\hline Diabetes Mellitus & 0.55 & -38 & - & - \\
\hline Hypercholesterolemia & 0.55 & -31 & - & - \\
\hline Hypertension & 0.48 & 53 & - & - \\
\hline Kidney disease & 0.60 & -52 & - & - \\
\hline Cerebrovascular disease & 0.42 & 59 & - & - \\
\hline Heart failure & 0.56 & -86 & - & - \\
\hline COPD & 0.28 & -82 & 0.16 & -112 \\
\hline Prior ER & 0.25 & -114 & 0.093 & -143 \\
\hline Prior OR & 0.42 & -128 & - & - \\
\hline Symptomatic leg & 0.42 & -45 & - & - \\
\hline TASC score & & & 0.61 & -6 \\
\hline TASC A & 0.61 & 2.6 & - & - \\
\hline TASC B & 0.39 & 0.39 & - & - \\
\hline TASC C & 0.18 & 0.18 & - & - \\
\hline TASC D & Reference & Reference & - & - \\
\hline
\end{tabular}

$A B I$ indicates ankle brachial index; $C O P D$, chronic obstructive pulmonary disease; $E R$, endovascular revascularization; $O R$, open revascularization. 


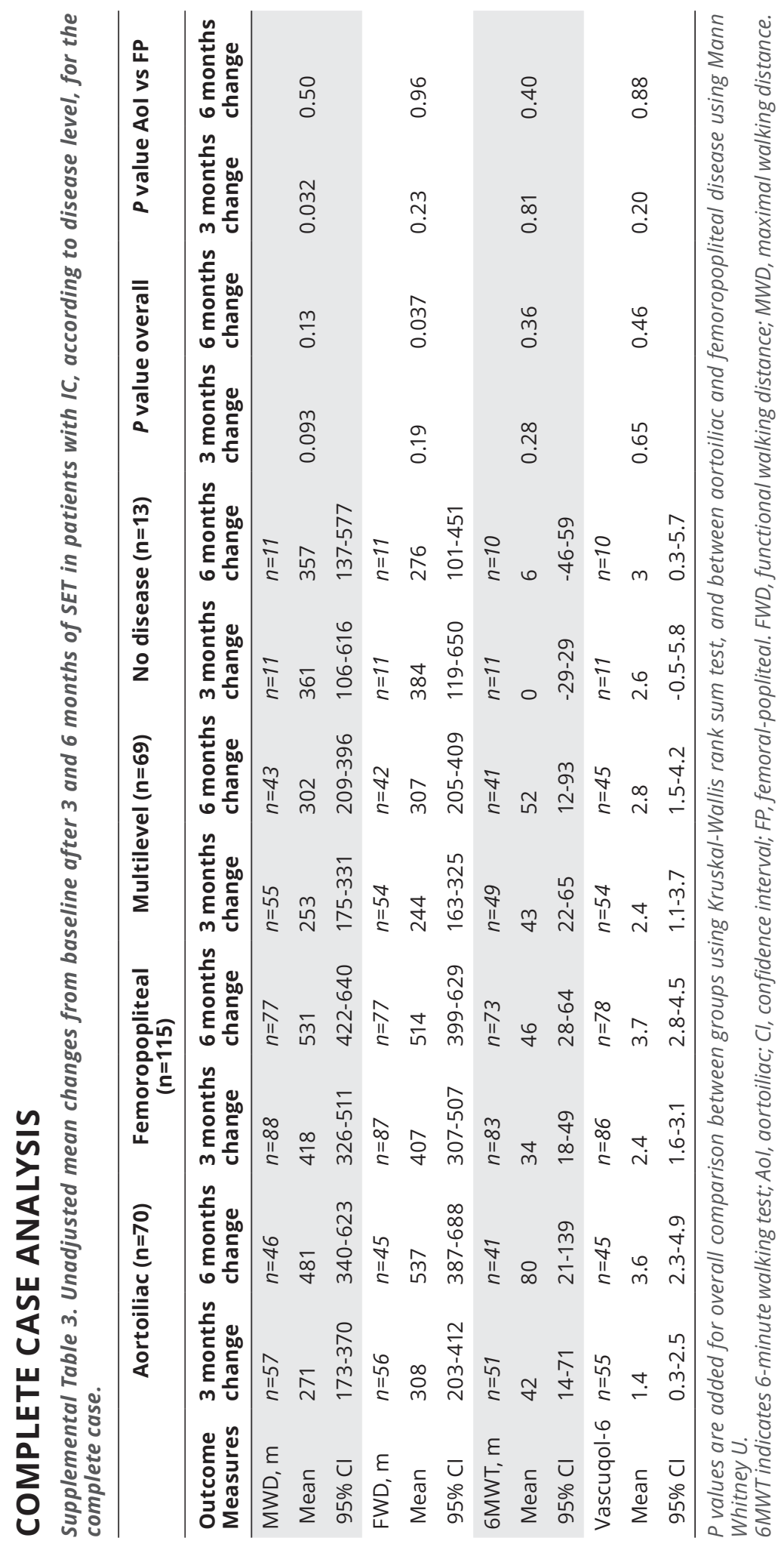




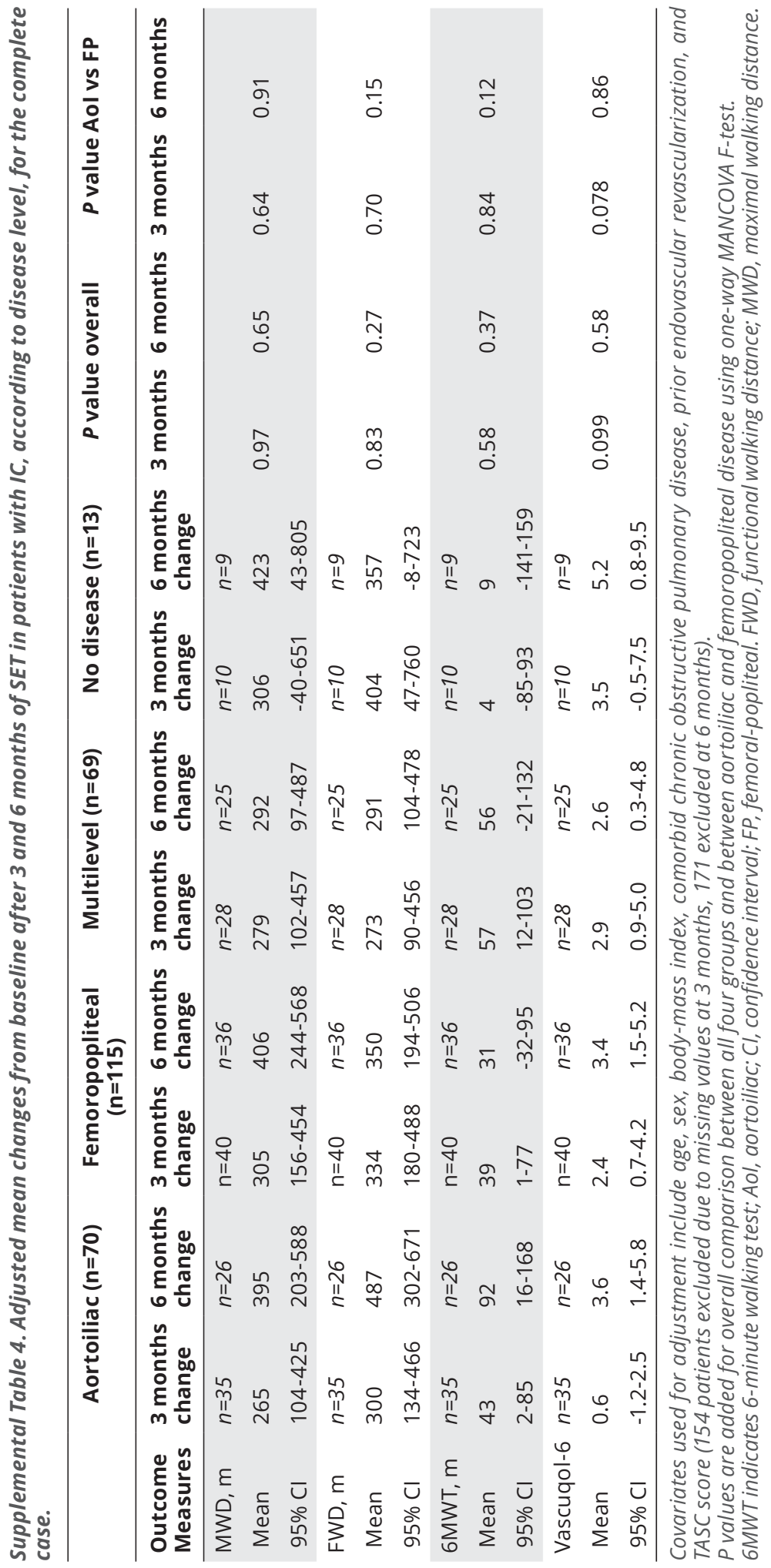




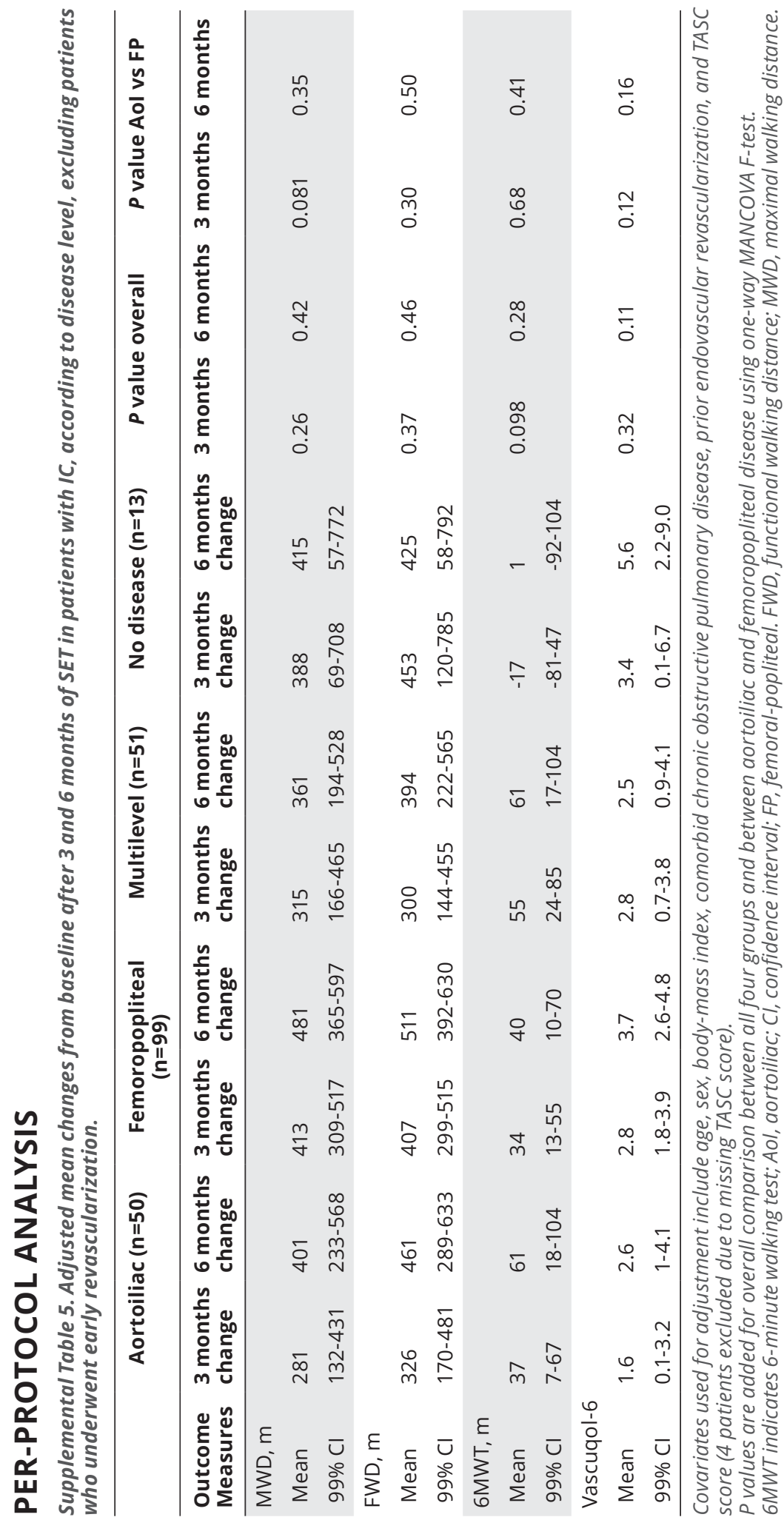




\section{INFLOW VERSUS OUTFLOW ANALYSIS}

Supplemental Table 5. Adjusted mean changes from baseline after 3 and 6 months of SET in patients with IC with inflow disease versus outflow disease.

\begin{tabular}{|c|c|c|c|c|c|c|}
\hline \multirow[b]{2}{*}{$\begin{array}{l}\text { Outcome } \\
\text { Measures }\end{array}$} & \multicolumn{2}{|c|}{$\begin{array}{l}\text { Inflow stenosis } \\
\qquad(n=137)\end{array}$} & \multicolumn{2}{|c|}{$\begin{array}{l}\text { Outflow stenosis } \\
\qquad(n=113)\end{array}$} & \multicolumn{2}{|c|}{ P value } \\
\hline & $\begin{array}{l}3 \text { months } \\
\text { change }\end{array}$ & $\begin{array}{l}6 \text { months } \\
\text { change }\end{array}$ & $\begin{array}{l}3 \text { months } \\
\text { change }\end{array}$ & $\begin{array}{l}6 \text { months } \\
\text { change }\end{array}$ & $\begin{array}{l}3 \text { months } \\
\text { change }\end{array}$ & $\begin{array}{l}6 \text { months } \\
\text { change }\end{array}$ \\
\hline \multicolumn{7}{|l|}{ MWD, m } \\
\hline Mean & 271 & 397 & 383 & 468 & 0.044 & 0.28 \\
\hline $95 \% \mathrm{Cl}$ & $186-357$ & $299-496$ & $289-478$ & $359-577$ & & \\
\hline \multicolumn{7}{|l|}{ FWD, m } \\
\hline Mean & 287 & 423 & 382 & 497 & 0.083 & 0.31 \\
\hline $95 \% \mathrm{Cl}$ & $198-375$ & $322-523$ & $284-480$ & $386-609$ & & \\
\hline \multicolumn{7}{|l|}{ 6MWT, m } \\
\hline Mean & 50 & 63 & 33 & 41 & 0.20 & 0.27 \\
\hline $95 \% \mathrm{Cl}$ & $30-69$ & $32-93$ & $12-55$ & $7-74$ & & \\
\hline \multicolumn{7}{|l|}{ Vascuqol-6 } \\
\hline Mean & 1.8 & 3.2 & 2.6 & 4 & 0.20 & 0.20 \\
\hline $95 \% \mathrm{Cl}$ & $0.8-2.7$ & $2.2-4.1$ & $1.6-3.7$ & $2.9-5.1$ & & \\
\hline
\end{tabular}

Covariates used for adjustment include age, sex, body-mass index, comorbid chronic obstructive pulmonary disease, prior endovascular revascularization, and TASC score (4 patients excluded due to missing TASC score).

$P$ values are added for comparison using one-way MANCOVA F-test.

6MWT indicates 6-minute walking test; Aol, aortoiliac; Cl, confidence interval; FP, femoral-popliteal. FWD, functional walking distance; MWD, maximal walking distance. 


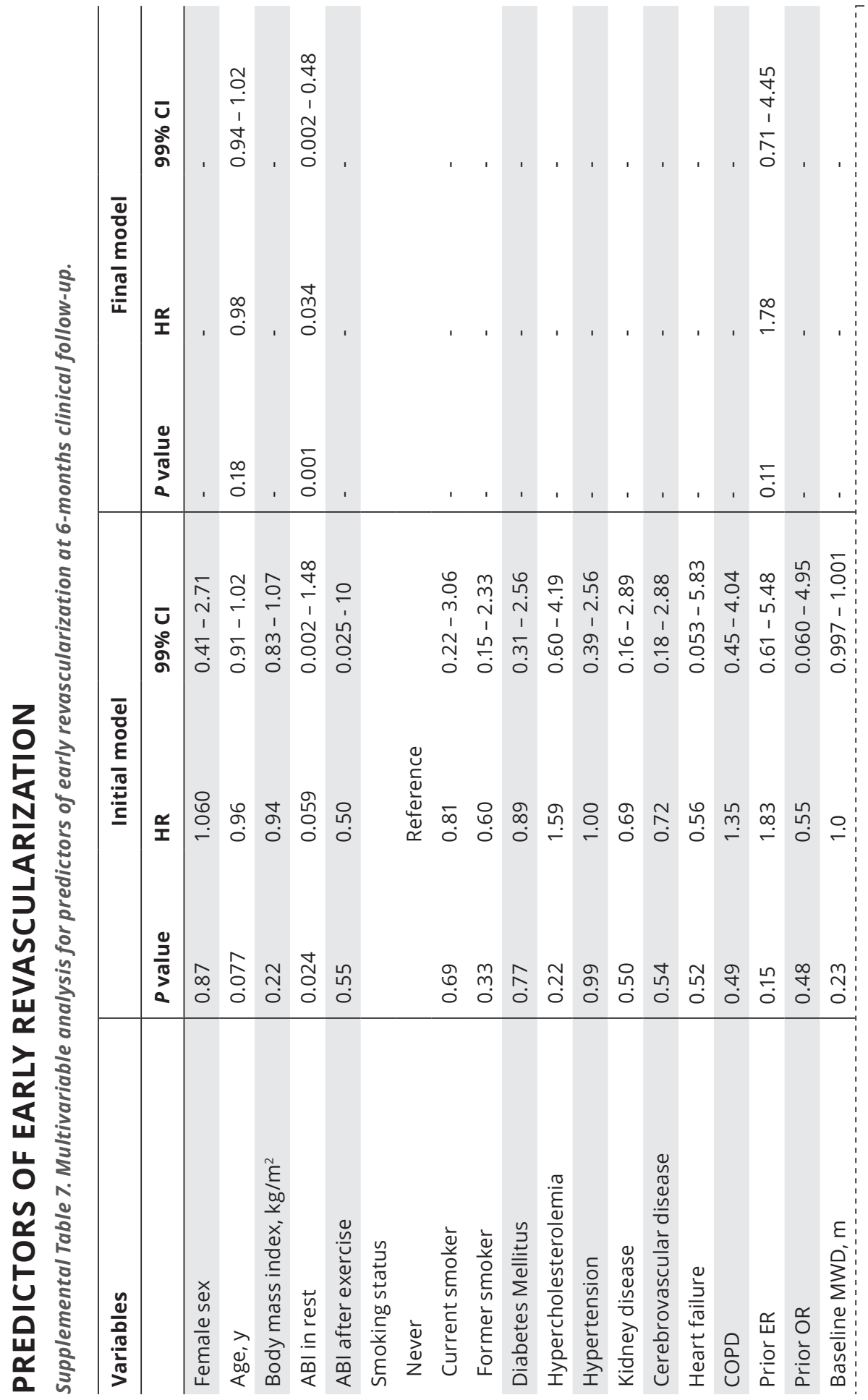




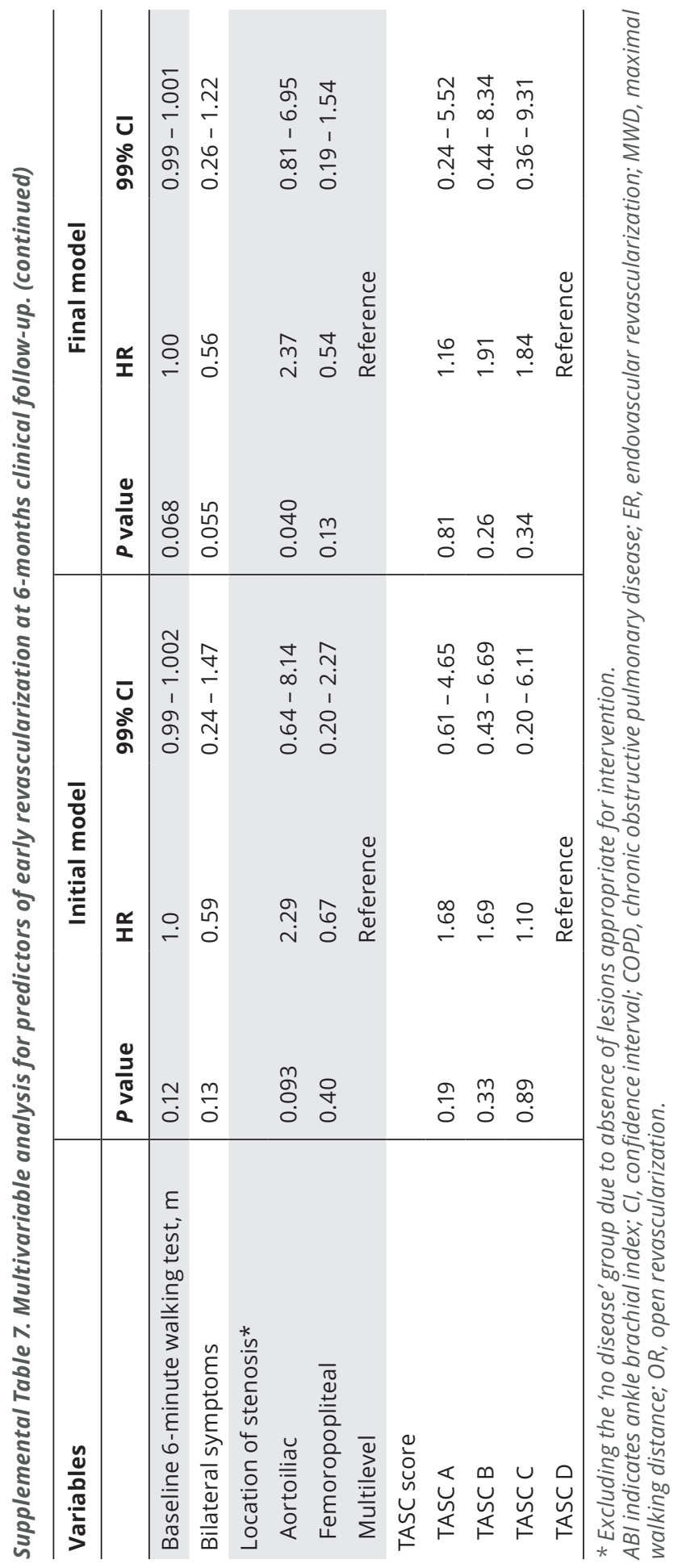





\title{
Chapter
}

\section{Supervised exercise therapy is effective for patients with intermittent claudication regardless of psychological constructs}

\author{
Jansen SCP \\ Hoeks SE \\ Nyklí̌̌ek I \\ Scheltinga MRM \\ Teijink JAW \\ Rouwet EV
}

Eur J Vasc Endovasc Surg. 2021 Dec 6;S1078-5884(21)00811-X. Online ahead of print. 


\section{ABSTRACT}

\section{Background}

According to current guidelines, supervised exercise therapy (SET) is the treatment of choice for intermittent claudication (IC). Little is known about the potential consequences of psychological factors on treatment effectiveness. This study aims to determine possible associations between a set of psychological constructs and treatment outcomes, and to investigate whether self-efficacy increased after SET.

\section{Methods}

This was a substudy of the ELECT Registry, a multicentre Dutch prospective cohort study in IC patients receiving primary SET. A complete set of validated questionnaires scoring extraversion, neuroticism, conscientiousness, anxiety, depression, selfcontrol, optimism and self-efficacy was obtained in 237 patients (median age 69yrs, $40 \%$ female). Anxiety and depression were dichotomized using established cut-off scores, whereas other scores were analysed as continuous measures. Multiple linear regression analyses determined possible associations between these independent variables and maximum and functional walking distances (MWD and FWD, respectively), Six-Minute Walk Test (6MWT), and VascuQol-6 (dependent variables). Self-efficacy during 12 months of SET was analysed using a linear mixed model.

\section{Results}

Neuroticism and anxiety were associated with lower overall VascuQol-6 scores (estimate -1.35 points (standard error (SE) 0.57), p .018 and -1.98 points (0.87), p .023; respectively). Optimism and self-efficacy demonstrated higher overall 6MWT (5.92 metres (2.34), p .012 and 1.35 metres $(0.42)$, p .001; respectively). Self-control was associated with lower overall log-MWD (-0.02 (0.01), p .038) and log-FWD (-0.02 (0.01), p .080), whereas self-efficacy with higher overall log-MWD ( 0.01 (0.003), p .009) and log-FWD (0.01 (0.003), p .011). Depressive IC patients demonstrated a larger improvement of 6MWT during follow-up (17.56 metres (8.67), p .044), but this small effect was not confirmed in sensitivity analysis. Self-efficacy did not increase during follow-up $(0.12 \%(0.49)$, p .080).

\section{Conclusions}

The beneficial effects of SET occur regardless of the psychological constructs, supporting current guidelines recommending a SET-first strategy in each IC patient. 


\section{INTRODUCTION}

The most common manifestation of peripheral artery disease (PAD) is intermittent claudication (IC), defined as exertional discomfort in leg muscles. Primary IC treatment goals are symptom relief and cardiovascular risk management. Standard treatment comprises of risk factor management, optimal medical therapy and supervised exercise therapy (SET) ${ }^{1,2}$ Lifestyle and psychosocial factors are known to play an important role in the prognosis of cardiovascular disease. ${ }^{3,4}$ Moreover, psychosocial risk factors may influence treatment outcomes as they act as potential barriers to treatment adherence and efforts to improve lifestyle. Therefore, assessment of such risk factors is recommended. ${ }^{4}$

However, psychosocial screening is not yet implemented in routine practice. To date, research on the potential influence of psychological constructs on the effectiveness of IC treatment is scarce. It may seem that depression and type D personality, which is related to neuroticism and introversion, negatively influence walking ability and quality of life. ${ }^{5,6}$ Additionally, possible associations between positive factors such as optimism and self-control and IC treatment outcomes are largely unknown. If certain psychological constructs indeed affect outcomes, IC treatment may require a tailored approach.

In addition, self-efficacy is an important mediator of behaviour change and is defined as one's judgement on being able to carry out a certain behaviour. It can be promoted through mastery experiences, social modeling, and motivational interviewing. ${ }^{7,8}$ It is currently unknown if SET increases self-efficacy.

This study aims to identify possible associations between a set of psychological constructs and treatment outcomes up to 6 months of follow-up in patients with IC, and to investigate whether self-efficacy increased during 12 months of SET.

\section{METHODS}

\section{Study design}

The present study is part of a multicentre prospective cohort study termed ELECT Registry. Participants were included between October 2017 and October 2018 in ten vascular surgery departments across the Netherlands. The study was approved by the Medical Research Ethics Committees United (reference number W17.071) and registered on the Dutch Trial Registry (trialregister.nl Identifier: NL7135 or NTR7332). Study methods were published previously. ${ }^{9}$ In summary, patients were eligible if diagnosed with IC (PAD Fontaine II/ Rutherford 1-3) and were considered candidates for SET as primary treatment. Exclusion criteria were advanced PAD stage (i.e. ischaemic rest pain and/or ulcers: Fontaine > II, Rutherford 4-6), vascular intervention as primary treatment, prior PAD treatment (SET or revascularisation) within 12 months before inclusion, or presence of a more dominant comorbidity. 
Primary objective of the ELECT Registry was to determine whether location of stenosis determined outcomes following SET as published. ${ }^{10}$

The present study objectives require different periods of follow-up. Possible associations between psychological constructs and treatment outcomes may be used for tailoring treatment strategy. As treatment evaluation occurs after 3 to 6 months, the 6 months follow-up point is most relevant in this context. Conversely, self-efficacy is essential to patients' perseverance in their efforts to improve health behaviour without intensive supervision from a physiotherapist and is preferably evaluated after 12 months follow-up.

\section{Treatment of subjects}

All patients received standard cardiovascular risk management and SET according to current guideline recommendations., ${ }^{1,2,11}$ A typical SET program entailed treadmillbased or track-based exercise and contains up to 37 individual 30 minutes sessions during a 3 to 12 months period. All physiotherapists were affiliated with ClaudicatioNet, the Dutch nationwide physiotherapist network specialised in IC treatment. ${ }^{12}$ They were all qualified to provide motivational interviewing and lifestyle coaching in addition to exercise therapy. A vascular surgeon evaluated treatment effect and possible need for revascularisation after 3 to 6 months of follow-up.

\section{Data collection}

The used diagnostic and outcome measures were recorded as part of standard care supplemented by a set of questionnaires. The participant's hospital record was used to document baseline characteristics, vascular laboratory data and treatment plan. Walking performance tests at baseline, 3, 6, and 12 months were collected by the physiotherapist. ${ }^{9}$

\section{Study measures}

Relevant treatment outcomes were functional walking distance (FWD), maximum walking distance (MWD), Six-Minute Walk Test (6MWT), Vascular Quality of Life Questionnaire-6 (VascuQoL-6), and freedom from lower leg vascular interventions. The psychological constructs of interest were personality traits of extraversion, neuroticism, conscientiousness, symptoms of anxiety and depression, and positive factors of self-control, optimism, and self-efficacy (see Supplemental Methods for definitions and reliability and consistency scores). These were all measured at baseline by means of validated Dutch translated questionnaires. Notably, selfefficacy was also determined at 3, 6, and 12 months following SET initiation.

\section{Big Five Inventory (BFI)}

The BFI consists of 44 items regarding statements of characteristics associated with five main personality traits. ${ }^{131415}$ Only extraversion, neuroticism and 
conscientiousness were examined, as two (agreeableness and openness) were found less relevant in the present context.

\section{Hospital Anxiety and Depression Scale (HADS)}

The HADS is a 14-item scale including 7 items on anxiety and 7 items on depression, both with a score ranging from 0 to $21 .{ }^{16,17} \mathrm{~A} \geq 8$ cut-off score is considered to reflect clinically relevant symptoms of anxiety and depression. ${ }^{18}$

\section{Brief Self-Control Score (BSCS)}

The Brief Self-Control Score determines self-control scoring 13 items with a fivepoint Likert scale. ${ }^{19,20}$ Scores range from 13 to 65 , whereas a high score is associated with low self-control.

\section{Life Orientation Test-Revised (LOT-R)}

The LOT-R measures optimism by means of 10 items rated on a five-point Likert scale. ${ }^{21,22}$ The total score ranges between 0 and 24 , and a high total score is associated with a high level of optimism.

\section{Barrier self-efficacy scale (BSES)}

The Barrier self-efficacy scale measures self-efficacy. It contains 13 items describing the possibility that one would indeed exercise despite potential barriers, such as bad weather or lack of interest. ${ }^{23}$ Inventors of this self-efficacy scale identified these barriers by attributive analysis of earlier participants' arguments for discontinuing exercise programs. Participants indicate their degree of self-perceived capability for each item on a 0 (no confidence at all) to $100 \%$ (complete confidence) scale. The mean percentage of all items yields a total score. A high score is associated with high barrier self-efficacy. This study used a validated Dutch translation, although one question ('My work schedule conflicted with my exercise session') was left out because the vast majority of participants was retired. ${ }^{24}$

\section{Statistical analysis}

Analyses were performed using R statistical software (version 3.6.2, December 2019). ${ }^{25}$ Categorical variables were presented as counts with percentages, continuous variables as means $\pm S D$, or as medians with interquartile ranges (IQR) in case of non-normal distribution. Distribution of continuous outcomes was assessed using visual inspection of plots and Shapiro-Wilk normality test. A p value $<.05$ was considered statistically significant. Sample size was only calculated for the primary objective of the ELECT Registry, as previously published..$^{9,10}$

Multivariable linear regression was performed using baseline psychological constructs as independent variables and SET-related outcomes as dependent variables. The model analysed both overall effects (i.e. lowered or raised the overall curve) and interaction effects with follow-up time (i.e. course during follow- 
up). Using random intercepts and random slopes, the model respected individual differences at baseline and possible variation in associations between individuals. In case of nonnormal distribution of residuals, log transformation was used. HADS' sub scores were dichotomized using standardized cut-off scores ( $\geq 8$ for anxiety and depressive symptoms). Other psychological construct scores were analysed as continuous measures. The following covariates were included in adjusted analyses: age, sex, smoking, dyslipidaemia, BMI, hypertension, diabetes, cardiovascular disease (i.e. myocardial infarction, angina, heart failure), cerebrovascular disease (i.e. transient ischemic attack, cerebrovascular incident), pulmonary disease (i.e. chronic obstructive pulmonary disease or other pulmonary disease), renal insufficiency, lower extremity musculoskeletal disease, prior lower extremity revascularisation, prior SET, location of stenosis, resting ankle-brachial index (ABI), and ABI after exercise. A generalized linear model was used to analyse possible associations between baseline psychological constructs and freedom from vascular intervention during follow-up. This analysis was only adjusted for age and sex due to ratio of number of events per variable. Whether self-efficacy increased during 12 months of follow-up was analysed using a linear mixed model with self-efficacy as dependent variable correcting for all covariates as mentioned earlier.

Missing data, if not completely at random, were multiply imputed to minimise bias. ${ }^{26}$ Multiple imputation by chained equations (MICE) was performed under the assumption of missing at random (MAR), using MICE package in R. ${ }^{27}$ The outcome was included in the imputation model. Sensitivity analyses involved complete case analyses including only cases in which all relevant outcomes and variables were completely available.

\section{RESULTS}

A total of 297 patients were included in the ELECT Registry of which 237 patients completed the baseline psychological construct questionnaires (Figure 1). Not completing the questionnaires was mostly due to logistical reasons. However, sensitivity analysis demonstrated that outcomes of participating and nonparticipating groups were not different. Loss to follow-up concerned 27 patients at 3 months of follow-up, and an additional 20 patients at 6 months. There were no missing data for the psychological questionnaires at baseline, as this was an inclusion criterion for the study. The numbers of available data before imputation are described in Figure 1 for MWD, FWD, 6MWT and VascuQol- 6 at baseline and during follow-up. Regarding baseline characteristics, data were complete except for BMI (42\% missing), ankle brachial index (3\%) and affected vascular segment (7\%). Characteristics are presented in Tables 1 and 2. One in 5 patients (21\%) had clinically relevant symptoms of anxiety and one in $6(16 \%)$ of depression. 


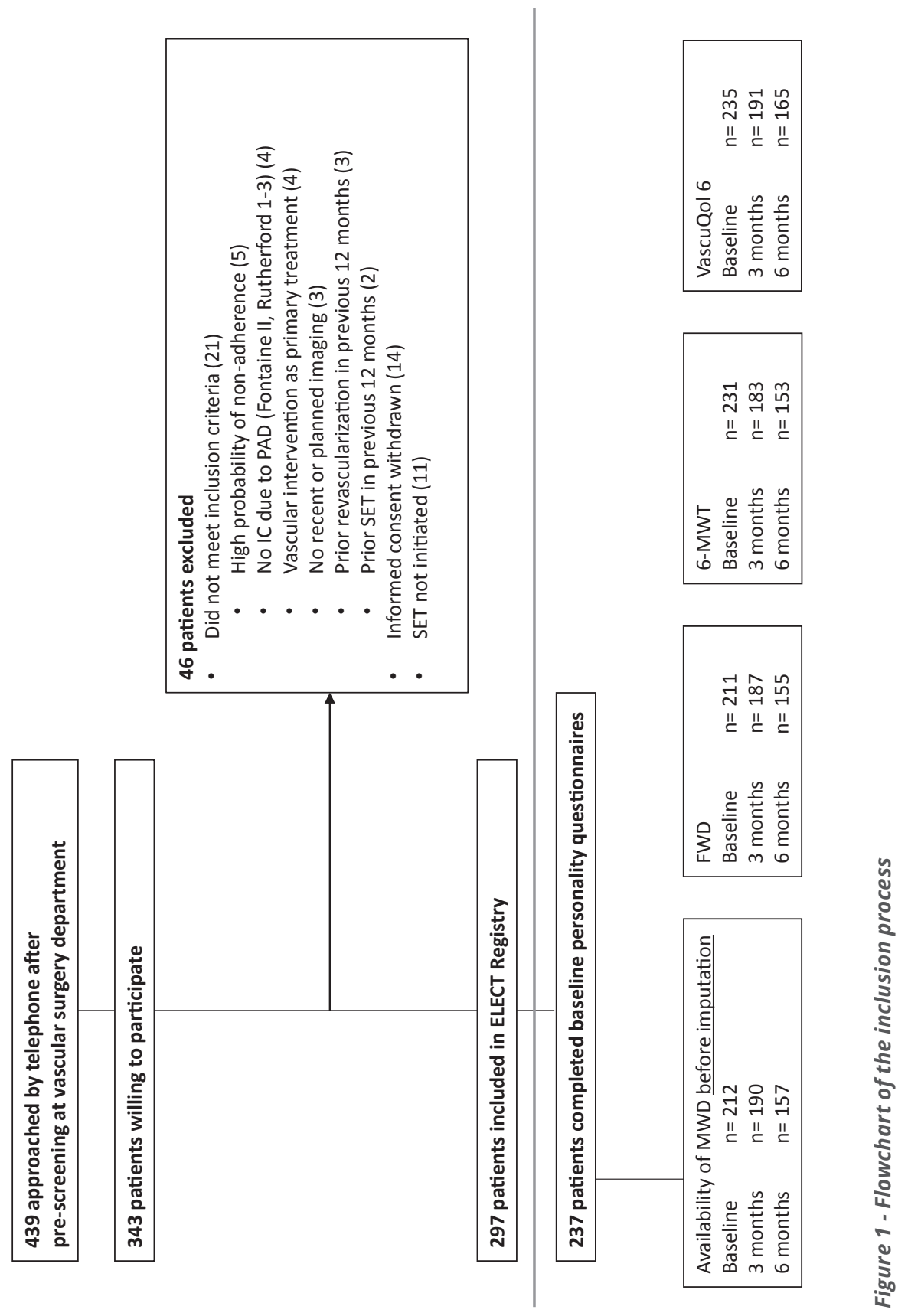


Table 1. Baseline characteristics

\begin{tabular}{|c|c|}
\hline \multicolumn{2}{|l|}{ Number of participants: 237} \\
\hline Age, median (IQR), years & $69(63-74)$ \\
\hline Female sex, No. (\%) & $95(40)$ \\
\hline \multicolumn{2}{|l|}{ Smoker, No. (\%) } \\
\hline Current & $101(43)$ \\
\hline Former & $100(42)$ \\
\hline Dyslipidaemia, No. (\%) & $132(56)$ \\
\hline Body mass index, median (IQR) ${ }^{1}$ & $26.6(24.2-29.4)$ \\
\hline Hypertension, No. (\%) & $147(62)$ \\
\hline \multicolumn{2}{|l|}{ History of disease, No (\%) } \\
\hline Diabetes & $65(27)$ \\
\hline Cardiac $^{2}$ & $55(23)$ \\
\hline Cerebrovascular ${ }^{3}$ & $28(12)$ \\
\hline Pulmonary ${ }^{4}$ & $63(27)$ \\
\hline Renal insufficiency & $27(11)$ \\
\hline Lower extremity musculoskeletal disease & $36(15)$ \\
\hline Prior lower extremity revascularization, No. $(\%)^{5}$ & $55(23)$ \\
\hline Prior supervised exercise therapy, No. $(\%)^{5}$ & $28(12)$ \\
\hline \multicolumn{2}{|l|}{ Affected vascular segment, No. (\%) } \\
\hline Aortoiliac & $55(24)$ \\
\hline Femoropopliteal & $96(40)$ \\
\hline Multilevel $^{6}$ & $60(25)$ \\
\hline Rest group ${ }^{7}$ & $10(4)$ \\
\hline Unknown ${ }^{8}$ & $16(7)$ \\
\hline \multicolumn{2}{|l|}{ Ankle brachial index, median (IQR) ${ }^{9}$} \\
\hline At rest & $0.60(0.50-0.80)$ \\
\hline After exercise & $0.30(0.20-0.40)$ \\
\hline \multicolumn{2}{|l|}{ Walking distance, median (IQR), metres } \\
\hline Maximum & $380(255-631)$ \\
\hline Functional & $240(145-400)$ \\
\hline 6-Minute walk test, median (IQR), metres & $380(306-440)$ \\
\hline VascuQol-6 score, median (IQR) & $16(12-18)$ \\
\hline
\end{tabular}

${ }^{1}$ Calculated as weight in kilograms divided by height in meters squared.

${ }^{2}$ Defined as myocardial infarction, angina or heart failure.

${ }^{3}$ Defined as transient ischemic attack or cerebrovascular accident.

${ }^{4}$ Defined as chronic obstructive pulmonary disease or other pulmonary disease.

${ }^{5}$ Excluding the 12 months prior to inclusion as defined in the exclusion criteria.

${ }^{6}$ Defined as the combination of aortoiliac and femoropopliteal level.

${ }^{7}$ Defined as no significant stenoses in aortoiliac and femoropopliteal tract.

${ }^{8}$ Although imaging was planned at time of inclusion, this was ultimately not obtained or imaging was only available for one level (aortoiliac or femoropopliteal).

${ }^{9}$ Minimum value for right and left legs. 


\section{SET-related outcomes}

MWD, FWD, 6MWT and VascuQol-6 improved after 6 and 12 months of followup (Table 3). Most SET sessions were followed in the first 3 months (median 17 sessions, IQR 14-20). After 6 and 12 months, this number increased to 26 sessions (21 - 30 sessions) and 34 sessions (27-37), respectively. Secondary to a seemingly unsuccessful SET, lower extremity revascularisation was provided to 40 patients (16.9\%) after 6 months, and to a total of 62 (26\%) after 12 months.

Table 2. Baseline psychological construct scores

\begin{tabular}{lll}
\hline Number of participants: $\mathbf{2 3 7}$ & & Possible range \\
\hline $\begin{array}{l}\text { BFI, median (IQR) } \\
\text { Extraversion }\end{array}$ & 1 to 5 \\
$\begin{array}{l}\text { Neuroticism } \\
\text { Conscientiousness }\end{array}$ & $2.62(2.25-3.00)$ \\
HADS, median (IQR) & $3.67(3.22-4.11)$ & \\
Anxiety subscale & & 0 to 21 \\
Depression subscale & $5.00(3.00-7.00)$ & \\
Self-control, median (IQR) & $3.00(2.00-6.00)$ & 13 to 65 \\
Optimism, median (IQR) & $35(30-39)$ & 0 to 24 \\
Barrier self-efficacy, median (IQR) & $66.70(50.00-77.50)$ & 0 to 100 \\
\hline
\end{tabular}

Table 3. Change from baseline scores for 6MWT, FWD, MWD, and VascuQol-6 at 6 and 12 months follow-up in patients with intermittent claudication primarily treated with supervised exercise therapy

\begin{tabular}{llllll}
\hline Outcome & $\begin{array}{l}\text { Baseline value } \\
\text { median (IQR) }\end{array}$ & $\begin{array}{l}\text { Change from } \\
\text { baseline scores } \\
\text { 6 months } \\
\text { estimate } \\
\text { (standard error) }\end{array}$ & $\begin{array}{l}\text { p-value } \\
\text { Change from } \\
\text { baseline scores } \\
12 \text { months } \\
\text { estimate } \\
\text { (standard error) }\end{array}$ & p-value \\
\hline $\begin{array}{l}\text { MWD } \\
\text { metres }\end{array}$ & $380(255-631.8)$ & $215(14.66)$ & $<.001$ & $163(10.17)$ & $<.001$ \\
$\begin{array}{l}\text { FWD } \\
\text { metres }\end{array}$ & $240(145-400)$ & $224(15.46)$ & $<.001$ & $172(10.80)$ & $<.001$ \\
$\begin{array}{l}\text { 6MWT } \\
\text { metres }\end{array}$ & $380(306-440)$ & $23(2.73)$ & $<.001$ & $18(2.47)$ & $<.001$ \\
$\begin{array}{l}\text { VascuQol-6 } \\
\text { 16(12-18) }\end{array}$ & $1.7(0.15)$ & $<.001$ & $1.2(0.10)$ & $<.001$ \\
\hline
\end{tabular}

Unadjusted linear regression analysis of complete cases

MWD maximum walking distance, FWD functional walking distance, 6MWT 6-minute walking test, VascuQol-6 vascular quality of life questionnaire 


\section{Psychological constructs and relevant SET-related outcomes at 6 months follow-up}

Psychological constructs in relation to outcomes after SET are depicted in Table 4. Several psychological constructs had an overall effect on outcomes (i.e. lowered or raised the overall curve, including baseline measurement). However, the course during follow-up was not influenced except for depression (as depicted by the interaction terms). Neuroticism and anxiety were associated with lower overall VascuQol-6. Optimism and self-efficacy were associated with higher overall 6MWT. Self-control was associated with lower overall log-MWD and log-FWD, while selfefficacy was associated with overall higher log-MWD and log-FWD. Patients with a depression score above the cut-off point showed greater improvement of 6MWT during follow-up. Sensitivity analysis did not lead to different conclusions, except that the only follow-up effect of depression on 6MWT lost its significance (Supplemental Table 1). No associations were found for freedom from vascularisation at 6 months follow-up (Table 5). 


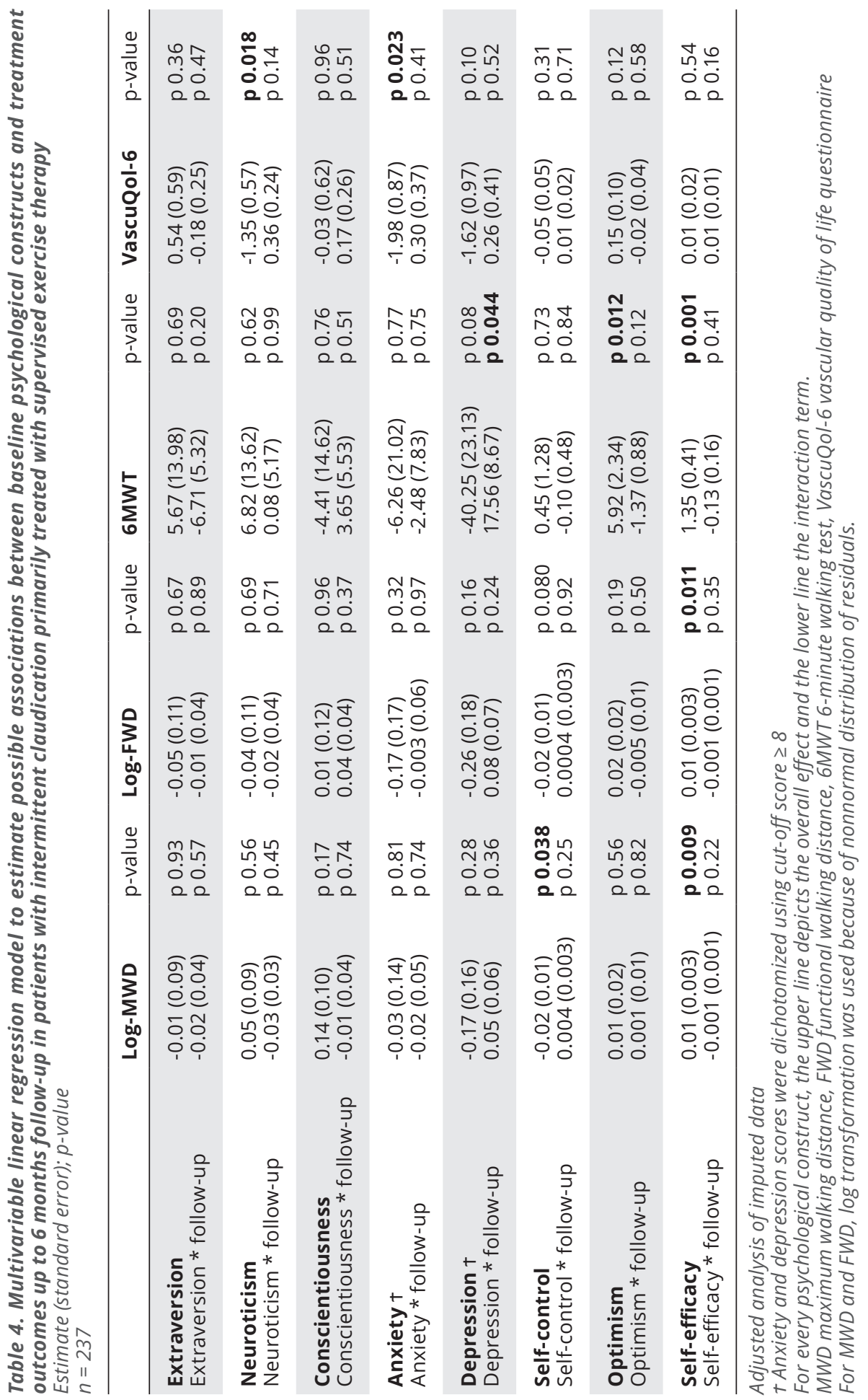


Table 5. Generalized linear model to estimate possible associations between baseline psychological constructs and freedom from revascularisation at 6 months follow-up in patients with intermittent claudication primarily treated with supervised exercise therapy $n=237$

\begin{tabular}{lll}
\hline & Estimate (standard error) & p-value \\
\hline Extraversion & $-0.32(0.30)$ & $\mathrm{p} 0.28$ \\
Neuroticism & $-0.20(0.28)$ & $\mathrm{p} 0.48$ \\
Conscientiousness & $-0.46(0.31)$ & $\mathrm{p} 0.14$ \\
Anxiety & $0.15(0.42)$ & $\mathrm{p} 0.72$ \\
Depression & $0.08(0.47)$ & $\mathrm{p} 0.86$ \\
Self-control & $0.02(0.03)$ & $\mathrm{p} 0.46$ \\
Optimism & $0.01(0.05)$ & $\mathrm{p} 0.91$ \\
\hline Self-efficacy & $-0.004(0.01)$ & $\mathrm{p} 0.67$ \\
\hline
\end{tabular}

Analysis of complete data adjusted for age and sex (no missing data)

\section{Self-efficacy during 12 months follow-up}

The self-efficacy questionnaire was completed by 188 patients at 3 months followup, 163 at 6 months, and 159 at 12 months follow-up. Median score at baseline was 66.70 (50 - 77.5), and slightly increased during follow-up (69.20 (55.4 - 87.5), 70 (54.6 - 83.3), and 70.8 (57.1 - 84.2) at 3, 6, and 12 months follow-up, respectively). Compared to baseline, the linear model demonstrated a small increase at 6 months follow-up (estimate $1.62(0.70)$, p .022), but no overall difference from baseline to 12 months of follow-up (0.12 (0.49), p .080) (see Figure 2).

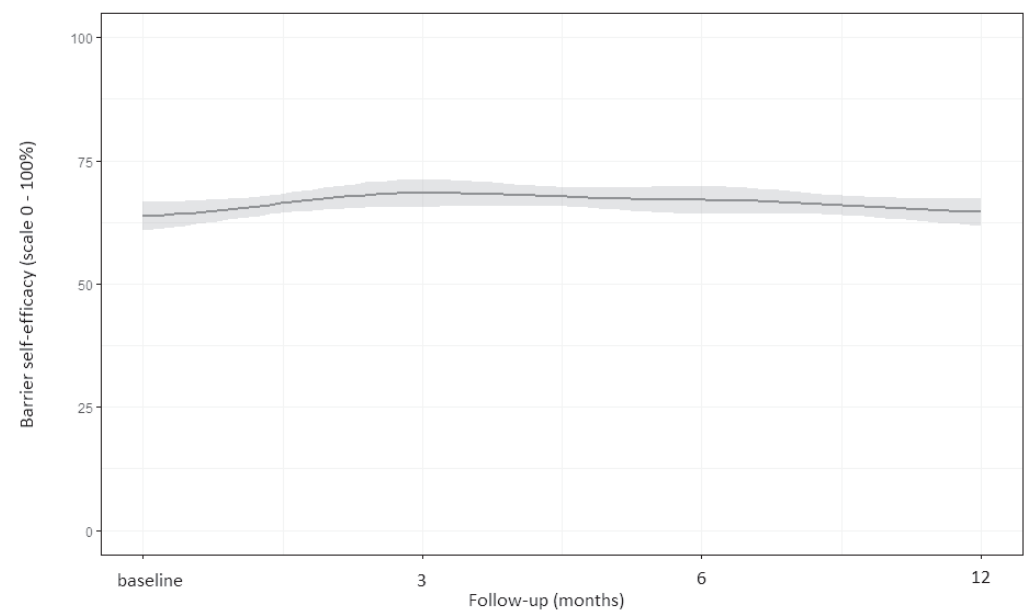

Figure 2 - Multivariable linear regression model of self-efficacy from baseline to 12 months follow-up.

$n=237$ 


\section{DISCUSSION}

This prospective cohort study demonstrates that SET improves walking performance and health-related quality of life in IC patients regardless of the investigated psychological constructs. Although several psychological factors demonstrated an overall effect on outcomes, they did not affect clinical course after 6 months followup. Patients with clinically relevant symptoms of depression improved more during follow-up, but this small effect disappeared after sensitivity analysis.

There is evidence supporting the contention that certain positive psychological factors are associated with improved outcomes in cardiovascular disease. For instance, optimism was related to superior physical activity and fewer cardiac readmissions in patients with acute coronary syndrome. ${ }^{28}$ In PAD patients, selfefficacy was positively associated with MWD and 6MWT. ${ }^{29}$ Furthermore, a metaanalysis found that general and exercise-specific self-efficacy have a positive effect on HR-QoL during cardiovascular rehabilitation. ${ }^{30}$ In the current study, patients with high self-efficacy and optimism demonstrated slightly higher overall 6MWT scores. As the minimally clinical important difference is 10 metres, these effects seem small and practical relevance may be doubted. ${ }^{31}$

Although patients with higher grades of neuroticism, anxiety and depression might present themselves with overall lower outcomes, they benefit at least as much from SET as other IC patients. The prevalence of anxiety and depression is higher in patients with PAD (percentages up to 48\%), and they more often develop depression during follow-up. 4,5,32,33 While some studies found more compromised physical function and walking ability in depressed PAD patients ${ }^{5,34}$, a recent systematic review found discrepant findings regarding the presence of anxiety and depression in relation to walking distances and QoL. ${ }^{6}$ The current study found lower overall VascuQol-6 scores for neuroticism and anxiety. Improvement in 6MWT during follow-up was certainly no less for patients with depressive symptoms than without. Although anxiety and depression do not clearly affect SET outcomes, it only seems reasonable to incorporate psychological screening as part of standard procedures in order to identify patients who need further psychological evaluation and assistance.

In the current study SET by itself does not increase self-efficacy. Limited mobility in PAD patients is often associated with lower expectations and self-efficacy, both promoting an inactive lifestyle. ${ }^{35}$ Self-efficacy is a key modifiable factor affecting cardiovascular rehabilitation participation and treatment outcomes. Increased selfefficacy is associated with improved cardiovascular lifestyle. ${ }^{30,36}$ An RCT in older patients found that an exercise program in itself was not sufficient to significantly increase barrier and exercise self-efficacy, and efficacy-levels even decreased after the program stopped. ${ }^{37}$ On the other hand, a group-mediated cognitive behavioural intervention in PAD patients improved self-efficacy independent of change in 6MWT. ${ }^{38}$ In the current population, barrier self-efficacy did not increase 
over time. Although SET includes mastery experiences, more attention should be paid to addressing patient-specific and situation-specific motivators and challenges to maintaining a routine walking program and physical activity. 7,38

The findings of this study support a recent statement indicating that there are no clinical patient characteristics that consistently identify individuals with IC who will not benefit from walking exercise. ${ }^{39}$ However, next to improving walking ability and reducing claudication symptoms, cardiovascular risk management is an essential part of IC treatment. While most studies focus on momentary walking distance, it is health behaviour such as physical activity that requires modification for achieving long-term benefit. Behaviour change is very challenging. Although several interventions are efficacious, even the most effective ones still have high levels of non-response. ${ }^{40}$ When designing health promotion programs for IC patients, future research may focus on distinguishing patients based on likelihood of response and to adjust intensity and type of counselling accordingly. ${ }^{41}$

\section{Limitations}

This study has limitations that should be addressed. First, the inclusion criteria allowed participation of most IC patients who visited the outpatient clinics. Although the included population was representative in terms of age, comorbidity and relevant baseline outcomes, relatively low ankle-brachial indices ${ }^{42,43}$ and high vascular intervention rates ${ }^{44}$ possibly reflected severe IC population. A portion of patients (20\%) declined participation which possibly introduced bias. Third, a considerable number of patients (20\%) were lost to follow-up. Missing data including follow-up outcomes were considered missing at random and were dealt with using multiple imputation techniques. There is no empirical method to check this assumption. Nevertheless, complete case analyses showed similar direction and uncertainty of effects. Fourth, this study was not powered to detect changes based on the psychological constructs. Finally, data on objective and reliable measurements of therapy compliance and physical activity levels were not obtained.

\section{CONCLUSION}

The beneficial effects of SET for IC occur regardless of certain psychological constructs including depression and anxiety. These findings support current guidelines recommending a SET-first strategy in each IC patient. Further research may focus interventions to increase self-efficacy and to promote sustainable effects.

\section{ACKNOWLEDGEMENTS}

The authors would like to express their appreciation to all participating patients and physiotherapists (affiliated with ClaudicatioNet) for their indispensable contribution to this study. Furthermore, we thank all outpatient clinic personnel involved in 
conducting the study who were not credited in the ELECT Study Group or as an author on this paper.

\section{The ELECT Study Group}

Catharina Hospital, Eindhoven: Joep AW Teijink, Marc RHM van Sambeek, Philippe WM Cuypers, Marijn ML van den Houten, Sandra CP Jansen, Anneroos Sinnige Amphia Hospital, Breda: Lijckle van der Laan, Gwan H Ho, Thijs MG Buimer Elisabeth Twee Steden Hospital, Tilburg: Patrick WHE Vriens Medical Spectrum Twente, Enschede: Edith M Willigendael VieCuri Medical Centre, Venlo: Jan-Willem M Elshof Rijnstate Hospital, Arnhem: Jan Willem P Lardenoije Maxima Medical Centre, Veldhoven: Marc RM Scheltinga Albert Schweitzer Hospital, Dordrecht: Maarten A Lijkwan University Medical Centre Utrecht, Utrecht: Eline S van Hattum Amsterdam University Medical Centres, Amsterdam: Mark JW Koelemay 


\section{REFERENCES}

1. Aboyans V, Ricco JB, Bartelink MEL, et al. ESC Guidelines on the Diagnosis and Treatment of Peripheral Arterial Diseases, in collaboration with the European Society for Vascular Surgery (ESVS). Eur J Vasc Endovasc Surg. 2017; 10.1016/j.ejvs.2017.07.018.

2. Gerhard-Herman MD, Gornik HL, Barrett C, et al. 2016 AHA/ACC Guideline on the Management of Patients With Lower Extremity Peripheral Artery Disease: Executive Summary: A Report of the American College of Cardiology/American Heart Association Task Force on Clinical Practice Guidelines. Circulation. 2017;135(12):e686-e725.

3. Rozanski A. Behavioral cardiology: current advances and future directions. $J$ Am Coll Cardiol. 2014;64(1):100-110.

4. Piepoli MF, Hoes AW, Agewall S, et al. 2016 European Guidelines on cardiovascular disease prevention in clinical practice: The Sixth Joint Task Force of the European Society of Cardiology and Other Societies on Cardiovascular Disease Prevention in Clinical Practice (constituted by representatives of 10 societies and by invited experts)Developed with the special contribution of the European Association for Cardiovascular Prevention \& Rehabilitation (EACPR). Eur Heart J. 2016;37(29):2315-2381.

5. Brostow DP, Petrik ML, Starosta AJ, Waldo SW. Depression in patients with peripheral arterial disease: A systematic review. Eur J Cardiovasc Nurs. 2017;16(3):181-193.

6. Sliwka A, Furgal M, Maga P, et al. The role of psychopathology in perceiving, reporting and treating intermittent claudication: a systematic review. Int Angiol. 2018;37(5):335-345.
7. Bandura A. Health promotion by social cognitive means. Health Educ Behav. 2004;31(2):143-164.

8. Miller, W., Rollnick, S. (2002). Motivational interviewing: Preparing people for change (2nd ed.). New York, NY: Guilford Press.

9. van den Houten MM, Jansen SC, Sinnige A, et al. Protocol for a prospective, longitudinal cohort study on the effect of arterial disease level on the outcomes of supervised exercise in intermittent claudication: the ELECT Registry. BMJ Open. 2019;9(2):e025419.

10. van den Houten $M M L$, Jansen $S$, van der Laan L, et al. The Effect of Arterial Disease Level on Outcomes of Supervised Exercise Therapy for Intermittent Claudication: A Prospective Cohort Study. Ann Surg. 2020; 10.1097/SLA.0000000000004073.

11. Merry AHH, Teijink JAW, Jongert MWA. KNGF-Richtlijn Symptomatisch Perifeer Arterieel Vaatlijden (In Dutch). 2017.

12. Hageman $D$, van den Houten MM, Spruijt S, Gommans LN, Scheltinga MR, Teijink JA. Supervised exercise therapy: it does work, but how to set up a program? J Cardiovasc Surg (Torino). 2017;58(2):305312.

13. John OP, Naumann LP, Soto CJ. Paradigm shift to the integrative Big Five trait taxonomy: History, measurement, and conceptual issues. In:John O.P., Robins R.W., Pervin L.A. (eds). Handbook of personality: Theory and research. . New York: Guilford Press; 2008:114-58.

14. Arterberry BJ, Martens MP, Cadigan JM, Rohrer D. Application of Generalizability Theory to the Big Five Inventory. Pers Individ Dif. 2014;69:98-103. 
15. Denissen JJ, Geenen R, van Aken MA, Gosling SD, Potter J. Development and validation of a Dutch translation of the Big Five Inventory (BFI). J Pers Assess. 2008;90(2):152-157.

16. Zigmond AS, Snaith RP. The hospital anxiety and depression scale. Acta Psychiatr Scand. 1983;67(6):361-370.

17. Bjelland I, Dahl AA, Haug TT, Neckelmann D. The validity of the Hospital Anxiety and Depression Scale. An updated literature review.J Psychosom Res. 2002;52(2):69-77.

18. Spinhoven P, Ormel J, Sloekers PP, Kempen GI, Speckens AE, Van Hemert AM. A validation study of the Hospital Anxiety and Depression Scale (HADS) in different groups of Dutch subjects. Psychol Med. 1997;27(2):363-370.

19. Tangney JP, Baumeister RF, Boone AL. High self-control predicts good adjustment, less pathology, better grades, and interpersonal success. J Pers. 2004;72(2):271-324.

20. Helmerhorst M, M. DVR. Zelf-controle Schaal en Zelf-Controle Schaal Observatie. Geautoriseerde Nederlandse vertaling van de Self-Control Scale (Tangney, Baumeister, Boone, 2004). Utrecht: Van der Hoeven Stichting, 2011.

21. Scheier MF, Carver CS, Bridges MW. Distinguishing optimism from neuroticism (and trait anxiety, selfmastery, and self-esteem): a reevaluation of the Life Orientation Test. J Pers Soc Psychol. 1994;67(6):1063-1078.

22. ten Klooster PM, Weekers AM, Eggelmeijer $F$, et al. Optimisme en/of pessimisme: factorstructuur van de Nederlandse life orientation test-revised. Psychologie en Gezondheid. 2010;38:89-100.
23. McAuley E. The role of efficacy cognitions in the prediction of exercise behavior in middle-aged adults. J Behav Med. 1992;15(1):65-88.

24. Stevens $M$, van Dijk $A B$, de Greef MHG, Lemmink KAPM, Rispens P. A Dutch translation of a questionnaire assessing self-efficacy in leisure-time physical activity. J Aging Phys Act. 2001;9:223-32.

25. R_Core_Team. R: A language and environment for statistical computing. 2017; https://www.R-project.org/. Accessed December 2019.

26. van der Heijden GJ, Donders AR, Stijnen T, Moons KG. Imputation of missing values is superior to complete case analysis and the missing-indicator method in multivariable diagnostic research: a clinical example. J Clin Epidemiol. 2006;59(10):1102-1109.

27. van Buuren S, Groothuis-Oudshoorn K. mice: Multivariate Imputation by Chained Equations in R. Journal of Statistical Software. 2011;45(3):1-67.

28. Huffman JC, Beale EE, Celano CM, et al. Effects of Optimism and Gratitude on Physical Activity, Biomarkers, and Readmissions After an Acute Coronary Syndrome: The Gratitude Research in Acute Coronary Events Study. Circ Cardiovasc Qual Outcomes. 2016;9(1):5563.

29. Collins TC, Lunos S, Ahluwalia JS. Selfefficacy is associated with walking ability in persons with diabetes mellitus and peripheral arterial disease. Vasc Med. 2010;15(3):189-195.

30. Banik A, Schwarzer R, Knoll N, Czekierda K, Luszczynska A. Self-efficacy and quality of life among people with cardiovascular diseases: A meta-analysis. Rehabil Psychol. 2018;63(2):295-312. 
31. Gardner AW, Montgomery PS, Wang M. Minimal clinically important differences in treadmill, 6-minute walk, and patientbased outcomes following supervised and home-based exercise in peripheral artery disease. Vasc Med. 2018;23(4):349357.

32. McDermott MM, Guralnik JM, Tian L, et al. Incidence and Prognostic Significance of Depressive Symptoms in Peripheral Artery Disease. I Am Heart Assoc. 2016;5(3):e002959.

33. Smolderen KG, Hoeks SE, Pedersen SS, van Domburg RT, de L, II, Poldermans D. Lower-leg symptoms in peripheral arterial disease are associated with anxiety, depression, and anhedonia. Vasc Med. 2009;14(4):297-304.

34. Smolderen KG, Aquarius $A E$, de Vries J, Smith OR, Hamming JF, Denollet J. Depressive symptoms in peripheral arterial disease: a follow-up study on prevalence, stability, and risk factors. J Affect Disord. 2008;110(1-2):27-35.

35. Rejeski WJ, Tian L, Liao Y, McDermott $M M$. Social cognitive constructs and the promotion of physical activity in patients with peripheral artery disease. $J$ Cardiopulm Rehabil Prev. 2008;28(1):65-72.

36. Sol BG, van der Graaf $Y$, van Petersen $R$, Visseren FL. The effect of self-efficacy on cardiovascular lifestyle. Eur J Cardiovasc Nurs. 2011;10(3):180-186.

37. McAuley E, Jerome GJ, Marquez DX, Elavsky S, Blissmer B. Exercise selfefficacy in older adults: social, affective, and behavioral influences. Ann Behav Med. 2003;25(1):1-7.

38. Rejeski WJ, Spring B, Domanchuk K, et al. A group-mediated, home-based physical activity intervention for patients with peripheral artery disease: effects on social and psychological function. J Transl Med. 2014;12:29.
39. Treat-Jacobson D, McDermott MM, Bronas UG, et al. Optimal Exercise Programs for Patients With Peripheral Artery Disease: A Scientific Statement From the American Heart Association. Circulation. 2019;139(4):e10-e33.

40. Kim ES, Kubzansky LD, Soo J, Boehm JK. Maintaining Healthy Behavior: a Prospective Study of Psychological WellBeing and Physical Activity. Ann Behav Med. 2017;51(3):337-347.

41. Kangovi S, Asch DA. Behavioral Phenotyping in Health Promotion: Embracing or Avoiding Failure. JAMA. 2018;319(20):2075-2076.

42. Murphy TP, Cutlip DE, Regensteiner $J G$, et al. Supervised exercise, stent revascularization, or medical therapy for claudication due to aortoiliac peripheral artery disease: the CLEVER study. J Am Coll Cardiol. 2015;65(10):999-1009.

43. Fakhry F, Spronk S, van der Laan L, et al. Endovascular Revascularization and Supervised Exercise for Peripheral Artery Disease and Intermittent Claudication: A Randomized Clinical Trial. JAMA. 2015;314(18):1936-1944.

44. Jansen SCP, van Nistelrooij LPJ, Scheltinga MRM, Rouwet EV, Teijink JAW, Vahl AC. Successful Implementation of the Exercise First Approach for Intermittent Claudication in the Netherlands is Associated with Few Lower Limb Revascularisations. Eur J Vasc Endovasc Surg. 2020;10.1016/j.ejvs.2020.07.074. 


\section{SUPPLEMENTAL METHODS -PSYCHOLOGICAL CONSTRUCTS}

This supplement provides definitions for the psychological constructs used in this study and also includes reliability and consistency scores for the used instruments.

\section{Big Five Inventory (BFI)}

The BFI focuses on personality traits, that reflect individual differences in patterns of behaviour, emotion and cognition. Each trait encompasses many lower-level characteristics (i.e. facets), as listed below.

Extraversion; orientation of one's interests and energy outward into the social world

- Facets: warmth, gregariousness, assertiveness, activity, excitement-seeking, positive emotion

- Opposite pole: introversion

Neuroticism; emotional instability and proneness to psychological distress

- Facets: anxiety, angry hostility, depression, self-consciousness, impulsiveness, vulnerability

- Opposite pole: emotional stability

Conscientiousness; tendency to be organized, persistent and motivated in goaldirect behaviour

- Facets: competence, order, dutifulness, achievement-striving, self-discipline, deliberation

- $\quad$ Opposite pole: unreliability

The BFI has a high test-retest reliability with intraclass correlation coefficients ranging from 0.93 to 0.96 . The Dutch translation that was used had a satisfactory reliability for measuring individual traits. Cronbach's alpha among traits are between 0.73 to 0.86 ( $a>0.7$ is a sign of good reliability).

\section{Hospital Anxiety and Depression Scale (HADS)}

The HADS is not an instrument to diagnose anxiety and depression, but merely indicates clinically relevant symptoms of anxiety and depression. The questions associated with the depressive component of the HADS were largely based upon the loss of pleasure response (i.e. anhedonia). The scale was specifically developed for use in patients suffering from somatic disease and therefore the questions do not refer to somatic symptoms such as pain and fatigue. 
Using the $\geq 8$ cut-off score, sensitivities and specificities for both subscales approximate 0.80 . Internal consistency ( 0.71 to 0.90 Cronbachs' alpha) and testretest reliability (0.91 Pearson correlation coefficient) are good.

\section{Brief Self Control Scale (BSCS)}

Self-control is the ability to be in command of one's behaviour and to restrain from impulses. The scale concerns self-discipline, non-impulsive behaviour, healthy habits, work ethic and reliability.

This scale has good test-retest reliability ( 0.87 Pearson correlation coefficient). The used Dutch translation has high internal consistency (Cronbachs' alpha 0.80).

\section{Life Orientation Test-Revised (LOT-R)}

The LOT-R measures optimism, but also contains items about pessimism that are scored in reverse. Optimism is a positive attitude about the future and can be viewed as a general way of thinking that can be a beneficial influence.

This test has satisfactory $(0.60$ to 0.68$)$ test-retest reliability. The used validated Dutch translation has moderate to high internal consistency (Cronbachs' alpha ranging from 0.58 to 0.80 ).

\section{Barrier Self-Efficacy Scale (BSES)}

Self-efficacy is defined as one's judgement on being able to carry out a certain behaviour. The BSES specifically measures self-efficacy in relation to exercise despite potential barriers.

Internal consistency of the Dutch scale is high (Cronbachs' alpha 0.84) and test-retest reliability is satisfactory ( 0.63 intraclass correlation coefficient). 


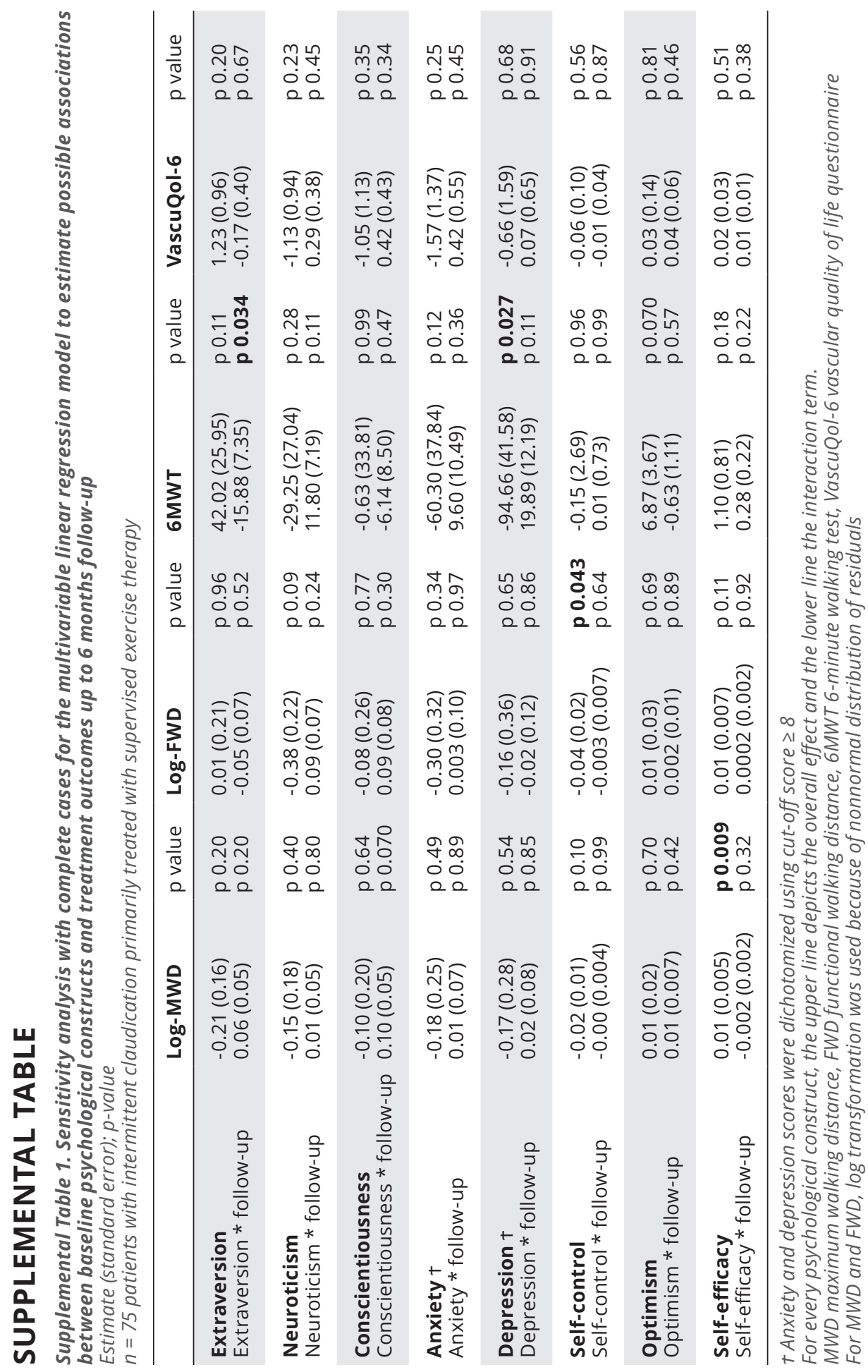





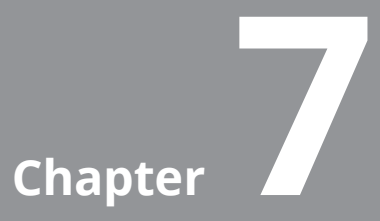

\title{
Modes of exercise training for intermittent claudication
}

\author{
Jansen SCP \\ Abaraogu UO \\ Lauret G] \\ Fakhry F \\ Fokkenrood HJP \\ Teijink JAW
}

Cochrane Database Syst Rev. 2020 Aug 20;8:CD009638 


\section{ABSTRACT}

\section{Background}

According to international guidelines and literature, all patients with intermittent claudication should receive an initial treatment of cardiovascular risk modification, lifestyle coaching, and supervised exercise therapy. In the literature, supervised exercise therapy often consists of treadmill or track walking. However, alternative modes of exercise therapy have been described and yielded similar results to walking. This raises the following question: which exercise mode produces the most favourable results? This is the first update of the original review published in 2014.

\section{Objectives}

To assess the effects of alternative modes of supervised exercise therapy compared to traditional walking exercise in patients with intermittent claudication.

\section{Search methods}

The Cochrane Vascular Information Specialist searched the Cochrane Vascular Specialised Register, CENTRAL, MEDLINE, Embase and CINAHL databases and World Health Organization International Clinical Trials Registry Platform and ClinicalTrials. gov trials registers to 4 March 2019. We also undertook reference checking, citation searching and contact with study authors to identify additional studies. No language restriction was applied.

\section{Selection criteria}

We included parallel-group randomised controlled trials comparing alternative modes of exercise training or combinations of exercise modes with a control group of supervised walking exercise in patients with clinically determined intermittent claudication. The supervised walking programme needed to be supervised at least twice a week for a consecutive six weeks of training.

\section{Data collection and analysis}

Two review authors independently selected studies, extracted data, and assessed the risk of bias for each study. As we included studies with different treadmill test protocols and different measuring units (metres, minutes, or seconds), the standardised mean difference (SMD) approach was used for summary statistics of mean walking distance (MWD) and pain-free walking distance (PFWD). Summary estimates were obtained for all outcome measures using a random-effects model. We used the GRADE approach to assess the certainty of the evidence. 


\section{Main results}

For this update, five additional studies were included, making a total of 10 studies that randomised a total of 527 participants with intermittent claudication (IC). The alternative modes of exercise therapy included cycling, lower-extremity resistance training, upper-arm ergometry, Nordic walking, and combinations of exercise modes. Besides randomised controlled trials, two quasi-randomised trials were included. Overall risk of bias in included studies varied from high to low. According to GRADE criteria, the certainty of the evidence was downgraded to low, due to the relatively small sample sizes, clinical inconsistency, and inclusion of three studies with risk of bias concerns. Overall, comparing alternative exercise modes versus walking showed no clear differences for MWD at 12 weeks (standardised mean difference (SMD) -0.01, 95\% confidence interval (CI) -0.29 to 0.27; P = 0.95; 6 studies; 274 participants; low-certainty evidence); or at the end of training (SMD $-0.11,95 \% \mathrm{CI}$ -0.33 to $0.11 ; P=0.32 ; 9$ studies; 412 participants; low-certainty evidence). Similarly, no clear differences were detected in PFWD at 12 weeks (SMD $-0.01,95 \% \mathrm{Cl}-0.26$ to $0.25 ; \mathrm{P}=0.97 ; 5$ studies; 249 participants; low-certainty evidence); or at the end of training (SMD $-0.06,95 \% \mathrm{Cl}-0.30$ to $0.17 ; \mathrm{P}=0.59 ; 8$ studies, 382 participants; low-certainty evidence). Four studies reported on health-related quality of life (HR-QoL) and three studies reported on functional impairment. As the studies used different measurements, meta-analysis was only possible for the walking impairment questionnaire (WIQ) distance score, which demonstrated little or no difference between groups (MD $-5.52,95 \% \mathrm{Cl}-17.41$ to 6.36 ; $\mathrm{P}=0.36 ; 2$ studies; 96 participants; low-certainty evidence).

\section{Authors' conclusions}

This review found no clear difference between alternative exercise modes and supervised walking exercise in improving the maximum and pain-free walking distance in patients with intermittent claudication. The certainty of this evidence was judged to be low, due to clinical inconsistency, small sample size and risk of bias concerns. The findings of this review indicate that alternative exercise modes may be useful when supervised walking exercise is not an option. More RCTs with adequate methodological quality and sufficient power are needed to provide solid evidence for comparisons between each alternative exercise mode and the current standard of supervised treadmill walking. Future RCTs should investigate outcome measures on walking behaviour, physical activity, cardiovascular risk, and HR-QoL, using standardised testing methods and reporting of outcomes to allow meaningful comparison across studies. 


\section{BACKGROUND}

\section{Description of the condition}

Peripheral arterial disease (PAD) is a chronic arterial occlusive disease caused by progressive atherosclerosis. Several arterial segments can be affected, such as the aorta and iliac, femoral, popliteal, and tibial arteries in the limbs. Approximately 202 million people are affected with PAD of the lower extremities, defined as an ankle-brachial index below 0.90 in either leg (Fowkes 2013). The incidence of PAD increases with age, in particular after the age of 40 years. In high-income countries, PAD affects $5.41 \%$ of men and $5.28 \%$ of women at the age of 45 to 49 years. This prevalence rises to $18.83 \%$ in men and $18.38 \%$ in women at the age of 85 to 89 years (Fowkes 2013). The most common symptom is intermittent claudication (IC), defined as a cramping pain in the muscles of the leg(s) that occurs during exercise and is relieved by a short period of rest. Because of this symptom, patients have a reduced painfree and maximum walking capacity, which restricts the patients' activity and mobility, leading to diminished health-related quality of life (Dumville 2004; McDermott 2001).

Moreover, given that PAD is a systemic disease which is characterised by generalised atherosclerosis, individuals with IC have an increased total risk of cardiovascular events beyond the claudication symptoms. Patients with IC have a five-year all-cause mortality rate of $10 \%$ to $15 \%$ and a $20 \%$ chance of a non-fatal cardiovascular event (Aboyans 2018). When IC progresses to critical limb ischaemia, an even higher mortality rate of $25 \%$ after one year is found (Conte 2015).

\section{Description of the intervention}

All patients with IC should receive a multicomponent therapy consisting of cardiovascular risk management, lifestyle counselling, and exercise therapy to reduce the associated serious health risks (Aboyans 2018; Gerhard-Herman 2017). Several randomised controlled trials and systematic reviews have compared walking exercise supervised by a physical or exercise therapist to non-supervised exercise, usual care, placebo, single walking advice, endovascular interventions, or bypass surgery (Fakhry 2018; Hageman 2018; Lane 2017; Parmenter 2015). The current evidence supports supervised exercise therapy as the primary treatment to improve walking capacity and health-related quality of life in patients with IC. Furthermore, community-based supervised exercise appears to be at least as efficacious as programmes provided in a hospital setting (Bendermacher 2007; Kruidenier 2009; Nicolaï 2010). Less attention has been paid to the mode of (supervised) exercise. Besides traditional walking, alternative modes of supervised exercise training, such as cycling, upper-extremity cycle ergometer exercise, Nordic walking, and strength training exist and are associated with a significantly improved walking capacity (Golledge 2018; Parmenter 2011; Tompra 2015). Notably, this update includes Nordic walking as an alternative exercise mode, in contrast to the original review in which 
Nordic walking was considered a different walking protocol (Lauret 2014). Recent research has provided new insights on the potential benefits of Nordic walking. This core-focused walking technique substantially differs from traditional walking, as it engages the muscles of the arms and trunk to reduce the load on the legs during walking (Golledge 2018).

Exercise training often provokes the claudication symptoms, depending on the protocol used (pain-free walking or walking until maximum pain). This can be considered as an adverse event, which is particularly relevant to patients in the context of expectation management. It generally takes several weeks for the patient to experience improvement and this type of treatment requires perseverance from the patient. Research has demonstrated that supervised exercise therapy is safe and has an exceedingly low all-cause complication rate (Gommans 2015).

\section{How the intervention might work}

Physical activity, which includes exercise, is associated with many health benefits (Piercy 2018). In patients with IC, exercise therapy significantly reduces symptoms and improves health-related quality of life. The exact mechanism for these benefits remains unclear and is probably multifactorial. A number of potential mechanisms have been suggested, such as induction of vascular angiogenesis, metabolic adaptations within skeletal muscle, improved endothelial flow-mediated dilatation, reduced inflammatory activation, reduced endothelial and mitochondrial dysfunction, improved walking efficiency, and increased pain tolerance (Conte 2015; Haas 2012; Harwood 2016). Additionally, supervised exercise therapy potentially reduces cardiovascular risk (Jansen 2019).

If walking ability is severely compromised by IC symptoms, traditional walking exercise may not provide sufficient stimulus to improve aerobic capacity. In those patients, alternative modes of aerobic exercise might be more beneficial than traditional walking. An example is Nordic walking, which is a core-focused walking technique which reduces the load on the legs during walking (Golledge 2018). Cycling is also a non-weight-bearing mode of exercise; and muscle strength seems to be associated with improved walking ability, which might explain the potential benefits of progressive resistance training of the lower limbs (Parmenter 2013b).

\section{Why it is important to do this review}

In most studies, supervised exercise programmes involve treadmill or track walking that is of sufficient intensity to provoke claudication symptoms. Walking exercise is alternated with rest over the course of a 30- to 60-minute session. Unfortunately, not all patients with IC are able to complete the exercise protocol because of concomitant comorbidity, such as arthrosis, chronic obstructive pulmonary disease, stroke, or cardiac complaints. For these patients, an adjusted protocol or alternative exercise regimen may be proposed. Being able to offer several modes of exercise might improve therapy adherence, as this enables better alignment with personal 
preferences. Combinations of aerobic and muscle-strengthening activities might be preferable, as these provide the most extensive health benefits according to the Physical Activity Guidelines (Piercy 2018).

This systematic review aims to determine the effect of alternative exercise modes on walking distance and health-related quality of life in patients with IC, compared to traditional walking exercise. Other Cochrane systematic reviews have demonstrated favourable results for supervised exercise programmes compared to usual care and non-supervised programmes (Hageman 2018; Lane 2017). We solely compared different exercise modalities between supervised programmes.

This is the first update of the original review published in 2014 (Lauret 2014). The original review concluded that there was no clear difference between supervised walking exercise and alternative exercise modes in improving the maximum and pain-free walking distance, but also highlighted the need for more studies with larger sample sizes. The international guidelines for the management of IC recommend considering the use of alternative exercise modes (such as cycling, upper-arm ergometry and strength training) to improve walking ability and functional status when walking is not an option for patients (Aboyans 2018; Gerhard-Herman 2017). In this update, we have presented all the currently available evidence on alternative exercise modes to help patients and healthcare providers determine which may offer the most benefit to them.

\section{OBJECTIVES}

To assess the effects of alternative modes of supervised exercise therapy compared to traditional walking exercise in patients with intermittent claudication.

\section{METHODS}

\section{Criteria for considering studies for this review}

\section{Types of studies}

We included parallel-group randomised controlled trials (RCTs) comparing (combinations of) alternative modes of exercise training (for example, ergometry, strength training, aerobic exercise, Nordic walking, etc.) with traditional walking exercise in patients with intermittent claudication (IC). Quasi-randomised trials were also included, but were distinguished from properly randomised trials in 'Risk of bias' assessment. We excluded cross-over, factorial, or cluster-RCTs. Types of participants We included studies where the study participants consisted of adults (18 years and older) with clinically determined IC (Fontaine II or Rutherford 1 to 3 (Aboyans 2018)), who were considered for conservative treatment. We excluded studies of participants with asymptomatic lower-limb atherosclerosis identified by testing. When studies described a mixture of asymptomatic and symptomatic 
participants, we contacted the authors to ask if a sub analysis was available. If not, we excluded these studies.

\section{Types of interventions}

We included all RCTs comparing alternative modes of exercise training (e.g. arm ergometry, strength training, cycling, Nordic walking, etc.) or combinations of exercise modes with a control group of traditional walking exercise. All exercise programmes had to be supervised at least twice a week during six consecutive weeks of training. We excluded studies reporting an exercise programme with a duration of less than six weeks of training or with less than two supervised sessions a week.

We excluded all types of mechanical intermittent compression treatments as we did not consider them to be exercise training. We excluded studies comparing different settings of walking exercise (supervised versus unsupervised, community versus hospital-based) or comparisons of different walking protocols (low- versus high-frequency training, low- versus high-intensity training, different treadmill exercise protocols).

\section{Types of outcome measures}

Studies were included only if reported outcome measures were available at baseline and after at least six weeks of follow-up.

\section{Primary outcomes}

- Maximal treadmill walking distance or time (MWD/T)

\section{Secondary outcomes}

- $\quad$ Pain-free treadmill walking distance or time (PFWD/T)

- Health-related quality of life scores (HR-QoL)

- $\quad$ Self-reported functional impairment (Walking Impairment Questionnaire)

\section{Search methods for identification of studies}

\section{Electronic searches}

The Cochrane Vascular Information Specialist conducted systematic searches of the following databases for randomised controlled trials and controlled clinical trials without language, publication year or publication status restrictions:

- Cochrane Vascular Specialised Register via the Cochrane Register of Studies (CRS-Web searched on 4 March 2019);

- Cochrane Central Register of Controlled Trials (CENTRAL) Cochrane Register of Studies Online (CRSO 2019, issue 2); 
- $\quad$ MEDLINE (Ovid MEDLINE® Epub Ahead of Print, In-Process \& Other NonIndexed Citations, Ovid MEDLINE $®$ Daily and Ovid MEDLINE ${ }^{\circledR}$ ) (searched from 1 January 2013 to 4 March 2019);

- $\quad$ Embase Ovid (searched from 1 January 2013 to 4 March 2019);

- $\quad$ CINAHL Ebsco (searched from 1 January 2017 to 4 March 2019);

- $\quad$ AMED Ovid (searched from 1 January 2017 to 4 March 2019).

The Information Specialist modelled search strategies for other databases on the search strategy designed for CENTRAL. Where appropriate, they were combined with adaptations of the highly sensitive search strategy designed by the Cochrane Collaboration for identifying randomised controlled trials and controlled clinical trials (as described in the Cochrane Handbook for Systematic Reviews of Interventions Chapter 6, Lefebvre 2011). Search strategies for major databases are provided in Appendix 1.

The Information Specialist searched the following trials registries on 4 March 2019:

- World Health Organization International Clinical Trials Registry Platform (who.int/trialsearch);

- ClinicalTrials.gov (clinicaltrials.gov).

\section{Searching other resources}

We searched the reference lists of relevant studies identified by the above search strategies to identify other relevant citations.

\section{Data collection and analysis}

\section{Selection of studies}

Two review authors (SJ and UA) independently selected studies for this review. Three review authors (GJ, HF, and JT) confirmed the suitability of selected studies for inclusion. Any disagreements regarding inclusion/exclusion of selected studies were solved through discussion.

\section{Data extraction and management}

Two review authors (SJ and UA) independently extracted data using a standard data collection form and entered data into Review Manager (Review Manager 2020). When necessary, additional information from included studies was sought by contacting study authors. The extracted study data consisted of the following:

- study characteristics, including study design, method of randomisation, exclusions post-randomisation, publication year, country, and study period;

- baseline characteristics, including number of participants, losses to followup, mean age, gender distribution, and inclusion and exclusion criteria; 
- type of interventions, including mode(s) of exercise, duration of programme, number of sessions, number of supervised sessions, and exercise protocol; and

- mean maximum walking distance or time, mean pain-free walking distance or time, mean quality of life scores at baseline and follow-up periods, and functional impairment information.

\section{Assessment of risk of bias in included studies}

Two review authors (SJ and UA) assessed the risk of bias for each study according to the checklist of design components, which comprised sequence generation, allocation concealment, blinding of personnel and participants, blinding of outcome assessments, incomplete outcome data, selective reporting, and other forms of bias. Another review author (GJ) confirmed the methodological quality of studies, primarily for adequacy of allocation concealment and follow-up. Due to the intervention (exercise), blinding of participants and personnel was not possible. Although this could introduce bias, we decided to consider the risk of bias to be low, since all studies experienced the same limitation. Consensus was achieved through informal discussion. The adequacy of each category was summarised as having 'low', 'unclear', or 'high' risk, according to criteria provided in the Cochrane Handbook for Systematic Reviews of Interventions (Higgins 2019).

\section{Measures of treatment effect}

We assessed treatment effects on the MWD and PFWD after 12 weeks follow-up and at the end of training. As studies with different treadmill test protocols and different measuring units (metres, minutes, or seconds) were included, the standardised mean difference (SMD) with 95\% confidence intervals (CI) approach was used for summary statistics of MWD and PFWD. The SMDs were interpreted by using rules of thumb for effect sizes, in which a SMD of 0.2 represents a small effect, 0.5 a moderate effect, and 0.8 a large effect (Cohen 1988). For HR-QoL and functional impairment scores, data were only pooled if more than one study used the same questionnaire. Therefore, the summary statistic was expressed as mean difference (MD) with $95 \% \mathrm{Cl}$.

\section{Unit of analysis issues}

We used the individual participant as the unit of analysis. Studies using cross-over design were not included in this review.

\section{Dealing with missing data}

Where applicable, missing data were requested from the authors. We expected that most studies reported baseline and final values with the corresponding standard deviations (SD). Where only change scores were provided, final value means were calculated using change score means and baseline data. Standard deviations 
were imputed using a conservative correlation coefficient of 0.5 for within-patient correlation from baseline to follow-up according to instructions in the Cochrane Handbook for Systematic Reviews of Interventions (Higgins 2019).

\section{Assessment of heterogeneity}

Statistical heterogeneity was assessed by the Chi²-test $(P<0.10$ considered as heterogeneous) and the $I^{2}$ statistic ( $I^{2}>50 \%$ considered as moderate to substantial risk of heterogeneity), in order to assess whether observed variation in trial results was compatible with the variation expected by chance alone (Higgins 2019). A rough guide to help interpretation of the $I^{2}$ statistic is as follows: $0 \%$ to $40 \%$ : might not be important; $30 \%$ to $60 \%$ : may represent moderate heterogeneity; $50 \%$ to $90 \%$ : may represent substantial heterogeneity; and $75 \%$ to $100 \%$ : considerable heterogeneity.

\section{Assessment of reporting biases}

No language restrictions were applied, to prevent language bias. In case of sufficient studies ( $\geq 10$ studies), we planned to assess publication bias with a funnel plot (Higgins 2019). If bias was present (demonstrated by an asymmetrical appearance of the funnel plot), the effect calculated by the meta-analysis would tend to overestimate the intervention effect.

\section{Data synthesis}

Data were analysed using Review Manager software (Review Manager 2020). We used final values, SDs, and number of participants to enable calculation of the SMD. The SDs were used to standardise the mean differences to a single scale and to compute study weights. It was assumed that between-study variation in SDs reflected only differences in measurement scales and not differences in the reliability of outcome measures or variability among study populations. As the alternative modes of exercise included in the current review consisted of several different types of exercise, we could not assume that intervention effects across studies were identical. Therefore, the DerSimonian and Laird random-effects model was used to analyse treatment effect, to account for between-study variance (DerSimonian 1986). The data of each study were summarised in forest plots and summary estimates with a $95 \% \mathrm{Cl}$ were calculated. A two-sided P (with a value $\leq 0.05$ ) was considered statistically significant.

\section{Subgroup analysis and investigation of heterogeneity}

Treatment effects were based on the outcomes after 12 weeks follow-up and at the end of training, in order to deal with heterogeneity in follow-up duration and because international guidelines recommend a programme duration of 12 weeks. Furthermore, we performed subgroup analyses of each type of alternative exercise mode and combinations of exercise modes, if more than one trial comparing the 
specific exercise mode or combinations of exercise to traditional walking was available.

\section{Sensitivity analysis}

We examined individual study effects by removing each study one at a time to check whether excluding a particular study significantly changed the results. In addition, we planned to perform sensitivity analyses on the methodological quality of the studies. Studies with apparent risk of bias were removed to examine whether excluding these studies significantly changed the results. Studies with more than one domain at high risk or more than three domains at unclear risk were considered to be at high risk of bias.

\section{Summary of findings and the assessment of certainty of the evidence}

We created a 'Summary of findings' table using GRADE-proGDT software (GRADEproGDT 2015) to present the main review findings for 'Alternative modes of exercise compared to walking exercise in people with intermittent claudication' (Summary of findings 1). We included the outcomes listed in Types of outcome measures. These were MWD, PFWD, HRQoL and functional impairment. The system developed by the Grades of Recommendation, Assessment, Development and Evaluation Working Group (GRADE working group) was used for grading the certainty of the evidence as high, moderate, low and very low, based on within-study risk of bias, directness of evidence, heterogeneity, precision of effects estimates, and risk of publication bias (Atkins 2004). 


\section{RESULTS}

\section{Results of the search}

See Figure 1.

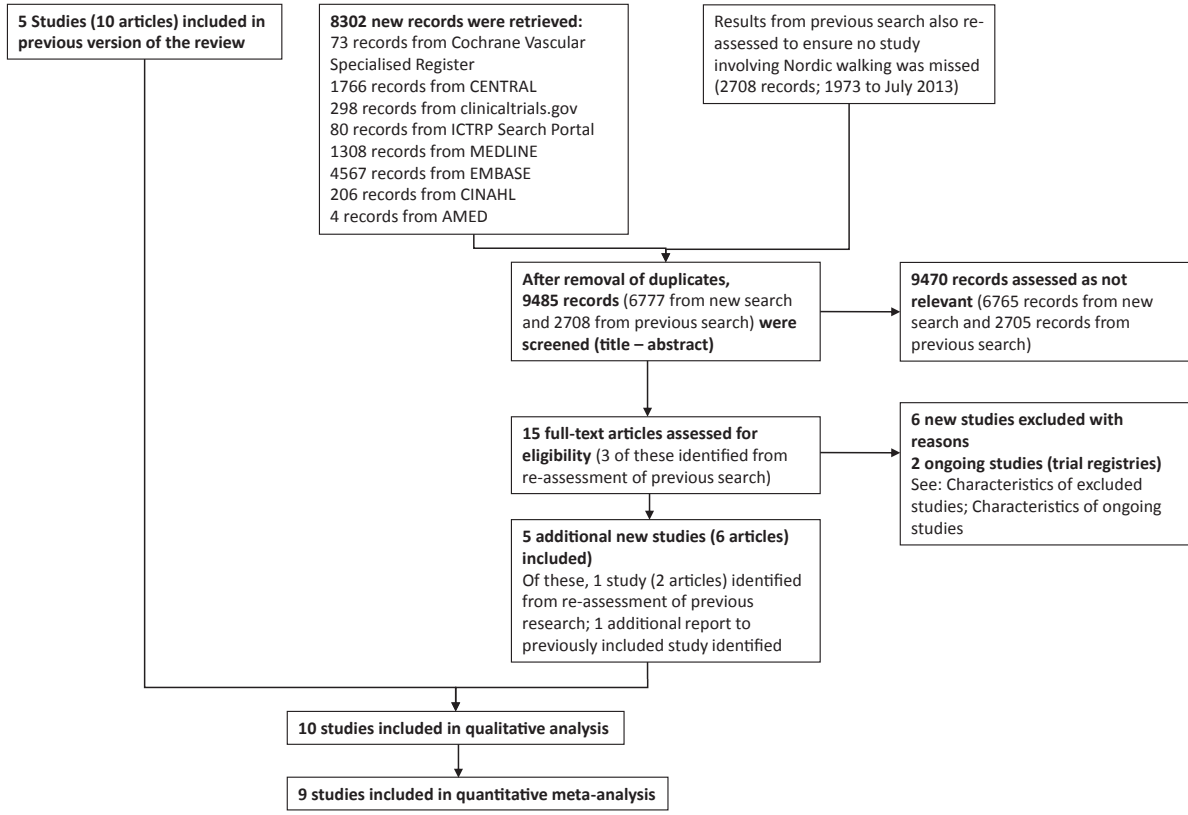

Figure 1 - Study flow diagram

For this update, the updated electronic searches identified 6777 new records (after removal of duplicates), of which four new studies were included in the review (Bulinska 2016; Delaney 2015; Kropielnicka 2018; Szymczak 2016). We also reevaluated the 2708 records identified in the first review published in 2014, according to the updated inclusion criteria (inclusion of Nordic walking as an alternative mode of exercise). This resulted in the inclusion of one additional study (Collins 2012). Two new ongoing studies were identified (ACTRN12616000243415; NCT03837639); and six new studies were excluded (Delaney 2014; EXERT Study 2018; Kao 2012; Oakley 2008; Parmenter 2013; Van Schaardenburgh 2017).

\section{Included studies}

For this update, five additional studies were included (Bulinska 2016; Collins 2012; Delaney 2015; Kropielnicka 2018; Szymczak 2016), making a total of 10 included studies (Bulinska 2016; Collins 2012; Delaney 2015; Kropielnicka 2018; McDermott 2009; Regensteiner 1996; Ritta-Dias 2010; Sanderson 2006; Szymczak 2016; TreatJacobson 2009). A summary of included studies is provided in the Characteristics of included studies tables. In these 10 studies, a total of 527 participants with 
intermittent claudication (IC) were randomised, of which 488 participants were randomised to the treatment arms that were relevant to this review. One study was relatively small involving fewer than 30 participants (Regensteiner 1996), six studies included more than 30 but fewer than 70 participants (Delaney 2015; McDermott 2009; Ritta-Dias 2010; Sanderson 2006;, Szymczak 2016; , Treat-Jacobson 2009); and three studies included 70 or more participants (Bulinska 2016; Collins 2012; Kropielnicka 2018). The mean age of the participants in the included studies varied between 62.0 and 73.4 years. All studies included both men and women, with the percentages of female participants ranging from $7 \%$ to $54 \%$. The studies were conducted in Australia (2), Brazil (1), Poland (3) and the United States (4). Nine studies compared supervised walking exercise to a single alternative mode of exercise, which was lower-extremity resistance training in four studies (McDermott 2009; Regensteiner 1996; Ritta-Dias 2010; Szymczak 2016), Nordic walking in three studies (Bulinska 2016; Collins 2012; Kropielnicka 2018), cycling (Sanderson 2006) and arm ergometry (Treat-Jacobson 2009). Four studies compared walking exercise to combination treatment, consisting of walking and lower-extremity resistance training (Delaney 2015; Regensteiner 1996), walking and arm ergometry (Treat-Jacobson 2009) and Nordic walking and lower extremity resistance training (Kropielnicka 2018).

Enrolment criteria were relatively homogeneous. In all studies, participants were included if a decrease in ankle brachial index ( $\mathrm{ABI}$ ) was present together with limiting or disabling symptoms of IC. Two studies (Collins 2012; McDermott 2009) assessed claudication symptoms by a questionnaire (Edinburgh Claudication Questionnaire and San Diego Claudication Questionnaire, respectively). McDermott 2009 included both symptomatic and asymptomatic patients with PAD, however only the data of participants with IC were included in this review. Delaney 2015 only included participants with radiographic evidence of infra-inguinal disease in the absence of aorta-iliac disease. In all studies, the presence of critical limb ischaemia was an exclusion criterion. Participants were also excluded if the exercise capacity was limited by another factor than IC (e.g. angina, chronic obstructive pulmonary disease, arthrosis) or if they were unable to perform the exercise programme (e.g. unable to walk on a treadmill or with Nordic poles). Szymczak 2016 excluded patients with diabetes and Kropielnicka 2018 excluded participants with "generally poor health". In four studies, claudication symptoms needed to be stable for three months (Kropielnicka 2018; Regensteiner 1996), six months (Ritta-Dias 2010), or more than 12 months (Sanderson 2006). Four studies excluded participants if a revascularisation procedure was performed in the previous year (Delaney 2015; Regensteiner 1996; Ritta-Dias 2010; Szymczak 2016). Szymczak 2016 excluded participants that suffered from stroke in the prior six months or had cardiac surgery in the prior 12 months. Three studies excluded participants if a coronary or lower-extremity revascularisation procedure was performed within the prior three months (Bulinska 2016; Kropielnicka 2018; Treat-Jacobson 2009). Two studies 
excluded participants in case of a cardiovascular event in the prior three months (Bulinska 2016); or 12 months (Kropielnicka 2018). One study excluded patients that participated in another exercise programme in the prior three weeks (Collins 2012). The two remaining studies excluded participants if they recently underwent surgery or a cardiovascular event (McDermott 2009; Sanderson 2006). Treatment duration varied between studies ranging from six weeks training (Sanderson 2006), to 12- week training (Bulinska 2016; Delaney 2015; Kropielnicka 2018; Regensteiner 1996; Ritta-Dias 2010; Szymczak 2016; Treat-Jacobson 2009) and 24-week training (Collins 2012; McDermott 2009).

\section{Excluded studies}

For this update, six additional studies (six publications) were excluded based on full-text assessment (Delaney 2014; EXERT Study 2018; Kao 2012; Oakley 2008; Parmenter 2013; Van Schaardenburgh 2017).

Overall, 23 studies were excluded based on full-text assessment. A summary of excluded studies is available in the Characteristics of excluded studies table. Three studies were excluded because they were not randomised (or quasirandomised) controlled trials (Gardner 2011; Kim 2006; Roitman 2010). Five studies were excluded because they were meeting posters with a limited description of the methods and results and no related articles were published or available after contact with the authors (Dedes 2010; Kao 2012; Kuwabara 2010; Ornelas 2011; Treat-Jacobson 2012). Twelve studies were excluded because they did not assess adequately supervised exercise therapy, according to the inclusion criteria of this review (Nawaz 2001; Oakley 2008; Parmenter 2013; Parr 2009; Saxton 2008; Saxton 2011; Van Schaardenburgh 2017; Tebbutt 2011; Treat-Jacobson 2012; Walker 2000; Wang 2008; Zwierska 2005). Six studies did not report the primary and secondary outcome measures of this review (Delaney 2014; Nawaz 2001; Saxton 2008; Saxton 2011; Treat-Jacobson 2011; Walker 2000). One study was excluded because the outcome measures were not clearly described and we did not receive additional information upon request(Jones 1996). The previous version of this review identified the EXERT study as an ongoing study (EXERT Study 2018). Although this study was completed, the original results have not been published. Only one publication was available comparing long-term follow-up (one to four years) to end of study results (24 weeks). Baseline data were not available from the authors, so we have now excluded this study.

\section{Ongoing studies}

Two ongoing studies were identified (ACTRN12616000243415; NCT03837639), for which a summary of study protocols is available in the Characteristics of ongoing studies table. One study (ACTRN12616000243415) has been completed, but the results have not yet been published or available from the authors (status: preparing the manuscript for submission to a peer-reviewed journal). 


\section{Risk of bias in included studies}

See Figure 2 and Figure 3.

Random sequence generation (selection bias)

Allocation concealment (selection bias)

Blinding of participants and personnel (performance bias): All outcomes Blinding of outcome assessment (detection bias): All outcomes

Incomplete outcome data (attrition bias): All outcomes Selective reporting (reporting bias) Other bias

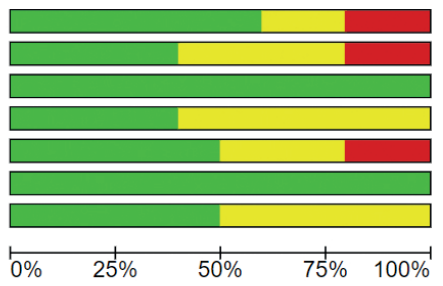

Low risk of bias Unclear risk of bias

High risk of bias

Figure 2 - Risk of bias graph: review authors' judgements about each risk of bias item presented as percentages across all included studies. 


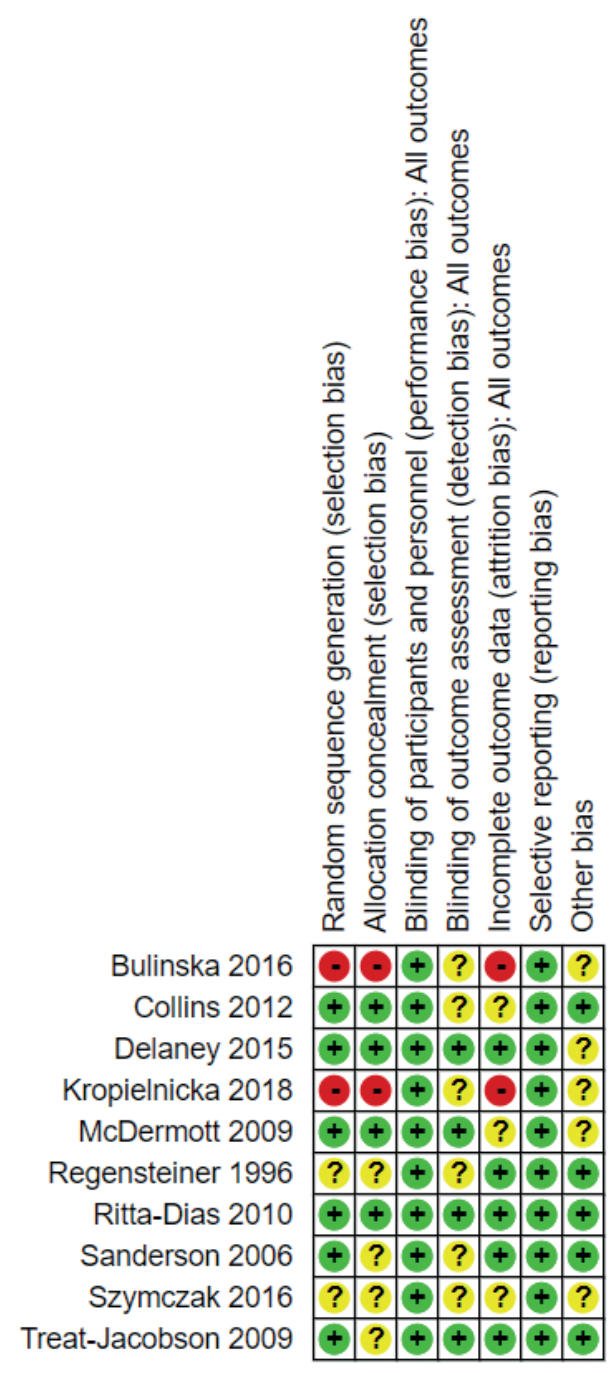

Figure 3 - 'Risk of bias' summary: review authors' judgements about each 'Risk of bias' item for each included study.

\section{Allocation}

Four studies described adequate sequence generation and allocation concealment by means of computer randomisation and so were at low risk of selection bias (Collins 2012; Delaney 2015; McDermott 2009; Ritta-Dias 2010). Two studies were judged to be at low risk for randomisation sequence bias but at unclear risk for allocation concealment as they described an adequate sequence generation, but did not describe the allocation concealment (Sanderson 2006; Treat-Jacobson 2009). Two studies were judged to have high risk of bias because they used a pseudo 
randomisation method in which participants were allocated by the investigators using a fixed block size (Bulinska 2016; Kropielnicka 2018). The two remaining studies did not describe the randomisation process and so were at an unclear risk of selection bias (Regensteiner 1996; Szymczak 2016).

\section{Blinding}

In all of the included studies, participants and personnel could not be blinded to the intervention (exercise). For this reason, bias could be introduced. However, since all studies experienced the same limitation, we considered the risk of bias to be low on this domain for all studies. Detection bias can be avoided by blinding the outcome assessors, which was achieved by four studies who were judged to be at low risk of bias (Delaney 2015; McDermott 2009; Ritta-Dias 2010; Treat-Jacobson 2009). The remaining studies did not provide information on blinding of outcome assessors and so were at unclear risk of detection bias (Bulinska 2016; Collins 2012; Kropielnicka 2018; Regensteiner 1996; Sanderson 2006; Szymczak 2016).

\section{Incomplete outcome data}

Five studies provided well-described reasons for missing data, which we considered to be plausible and well distributed among the groups and were at low risk of attrition bias (Delaney 2015; Regensteiner 1996; Ritta-Dias 2010; Sanderson 2006; Treat-Jacobson 2009). McDermott 2009 included both symptomatic and asymptomatic participants with PAD. We received the data for participants with IC upon request. Although the article described the missing data, it was unclear to what extent they were related to the group of symptomatic participants. Therefore, we considered the risk of attrition bias to be unclear. Two studies were judged to have high risk of attrition bias because missing data were not balanced across groups and no intention-to-treat analysis was performed (Bulinska 2016; Kropielnicka 2018). Bulinska 2016 did not provide reasons for drop-outs, while in the other study the majority of reasons was directly related to one of the interventions (Kropielnicka 2018). In Collins 2012, the risk of attrition bias was considered to be unclear, as the number of drop-outs was high and not evenly distributed among groups (18 versus 13). Szymczak 2016 did not provide information on incomplete outcome data, and so was also considered to be at an unclear risk of bias.

\section{Selective reporting}

We judged all studies to have low risk of reporting bias because published reports included all expected outcomes or because we obtained missing outcomes by contacting the authors (Bulinska 2016; Collins 2012; Delaney 2015; Kropielnicka 2018; McDermott 2009; Regensteiner 1996; Ritta-Dias 2010; Sanderson 2006; Szymczak 2016; Treat-Jacobson 2009). 


\section{Other potential sources of bias}

Since we included only nine studies in the quantitative analysis, we could not detect publication bias by using a funnel plot. We considered five studies to potentially have other sources of bias and so were judged as 'unclear' (Bulinska 2016; Delaney 2015; Kropielnicka 2018; McDermott 2009; Szymczak 2016). Bulinska 2016 reported significant differences in relevant baseline characteristics (hypertension and degenerative changes of spine and peripheral joints) which might have affected outcomes. Delaney 2015 excluded patients with radiographic aortic-iliac disease, but there is no scientific evidence that confirms a difference in treatment effects of exercise related to the level of disease. Therefore, this was also considered as a potential source of bias. McDermott 2009 included both asymptomatic and symptomatic participants with PAD. Although we only included data of symptomatic participants in this review, the article provided baseline characteristics for the total study population (including asymptomatic participants), which introduces a potential source of bias. In two other studies, baseline characteristics were either not clearly presented (Szymczak 2016) or very limited (Kropielnicka 2018). We identified no other potential sources of bias in the included studies.

\section{Effects of interventions}

Alternative modes of exercise training compared to traditional walking exercise after 12 weeks of training

\section{Maximum walking distance (MWD)}

Six studies reported the MWD after 12 weeks of training (Bulinska 2016; Kropielnicka 2018; Regensteiner 1996; Ritta-Dias 2010; Szymczak 2016; Treat-Jacobson 2009), with a total sample size of 274 participants. Overall, there was no clear difference detected between alternative modes of exercise compared to traditional walking (standardised mean difference (SMD) $-0.01,95 \% \mathrm{Cl}-0.29$ to 0.27 ; $\mathrm{P}=0.95$; lowcertainty evidence; see Analysis 1.1). Heterogeneity was considered low $(P=0.28$, $\mathrm{I}^{2}=18 \%$ ). See Summary of findings 1 . Sensitivity analysis was carried out by removing studies with high risk of bias, as defined in the Sensitivity analysis section (Bulinska 2016; Kropielnicka 2018; Szymczak 2016), and this had no effect on the SMD.

We investigated different modes of exercise by subgroup analysis (see Analysis 1.1). These are summarised below. No differences were detected by the test for subgroup differences $(P=0.16)$. 


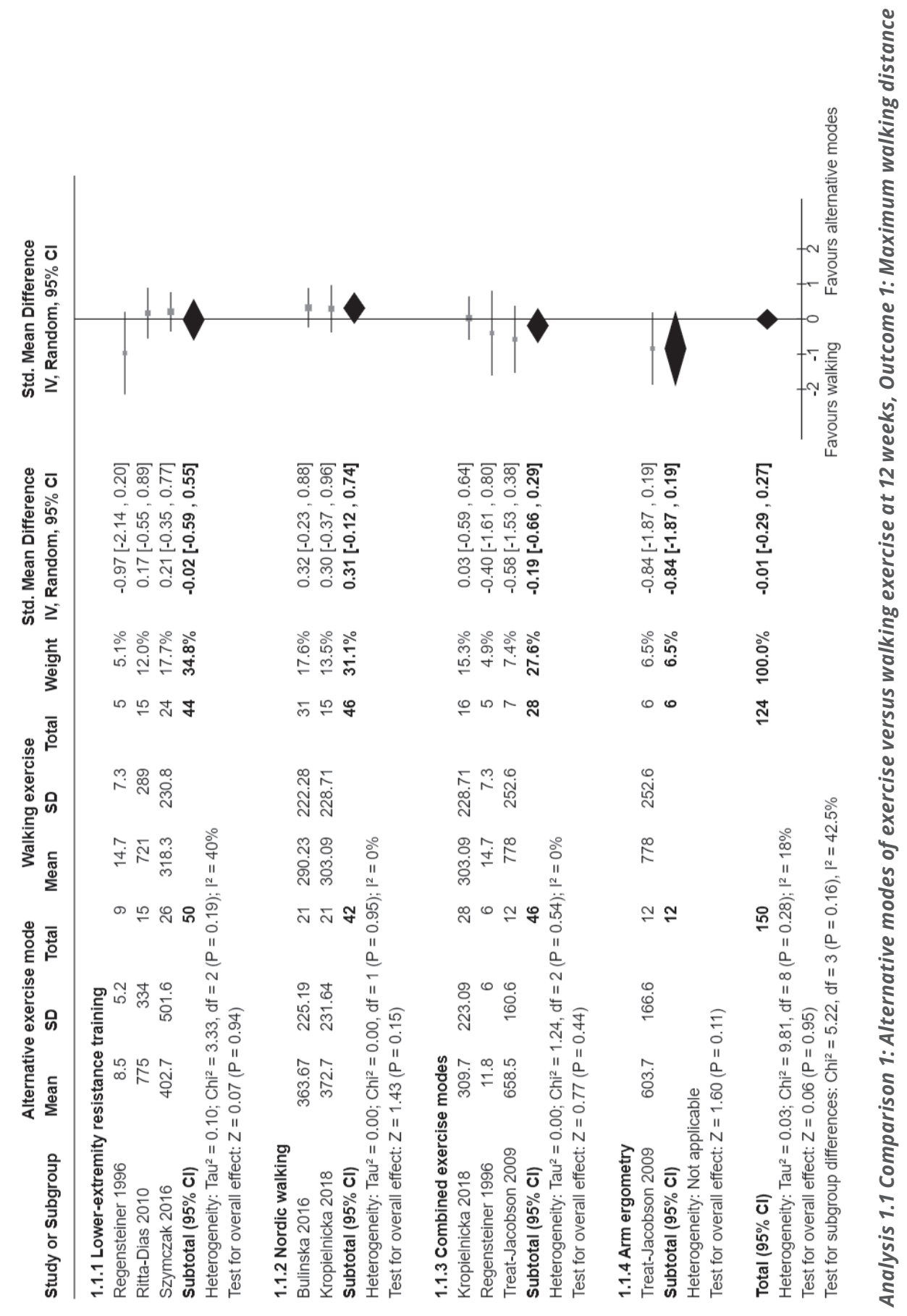


Lower extremity resistance training versus walking exercise Three studies reported the MWD after 12 weeks of training (Regensteiner 1996; Ritta-Dias 2010; Szymczak 2016), with a sample size of 94 participants. No difference was detected between the resistance and walking exercise groups (SMD $-0.02,95 \% \mathrm{Cl}-0.59$ to $0.55 ; \mathrm{P}=0.94$; Analysis 1.1). Heterogeneity was considered low $\left(I^{2}=40 \% ; P=0.19\right)$ and sensitivity analysis had no significant effect on the SMD.

Nordic walking versus walking exercise Two studies compared Nordic walking with walking and reported MWD after 12 weeks of training, with a sample size of 88 participants (Bulinska 2016; Kropielnicka 2018). The SMD of 0.31 represented little or no difference $(95 \% \mathrm{Cl}-0.12$ to $0.74 ; \mathrm{P}=0.15$; Analysis 1.1$)$. Heterogeneity was low $\left(I^{2}=0 \%\right)$.

Combination of exercise modes versus walking exercise Three studies with a sample size of 74 participants compared a combination of exercise modes with walking and reported MWD after 12 weeks of training (Kropielnicka 2018; Regensteiner 1996; Treat-Jacobson 2009). We found no clear difference for MWD (SMD -0.19, 95\% Cl -0.66 to $0.29 ; \mathrm{P}=0.44$; Analysis 1.1 ). Heterogeneity was low $\left(I^{2}=0 \%\right)$.

Arm ergometry versus walking exercise Only one small study investigated arm ergometry compared to walking exercise and no clear differences were detected in MWD between the groups (SMD -0.84, 95\% Cl -1.87 to 0.19; 18 participants; Analysis 1.1) (Treat-Jacobson 2009).

\section{Pain-free walking distance (PFWD)}

Five studies reported the PFWD after 12 weeks of training (Bulinska 2016; Kropielnicka 2018; Ritta-Dias 2010; Szymczak 2016; Treat-Jacobson 2009), with a sample size of 249 participants. Pooling did not detect a difference in PFWD between alternative modes and walking exercise (SMD $-0.01,95 \% \mathrm{Cl}-0.26$ to $0.25 ; \mathrm{P}=0.97$; low-certainty evidence; see Analysis 1.2). Heterogeneity was low $\left(I^{2}=0 \%\right)$. We carried out sensitivity analysis by removing studies with a high risk of bias, as defined in the Sensitivity analysis section (Bulinska 2016; Kropielnicka 2018; Szymczak 2016), and this had no effect on the SMD.

We investigated different modes of exercise by subgroup analysis (see Analysis 1.2). These are summarised below. No differences were detected by the test for subgroup differences $(P=0.39)$.

Lower extremity resistance training versus walking exercise Two studies reported the PFWD after 12 weeks of training, with a sample size of 80 participants (Ritta-Dias 2010; Szymczak 2016). There was no clear difference between groups for the pooled PFWD (SMD0.11, 95\% Cl-0.32 to 0.55; $\mathrm{P}=0.61$; Analysis 1.2). Heterogeneity was low $\left(I^{2}=0 \%\right)$.

Nordic walking versus walking exercise Two studies reported PFWD after 12 weeks of training, with a sample size of 88 participants (Bulinska 2016; Kropielnicka 
2018). No clear difference was detected between groups (SMD 0.14, 95\% Cl -0.28 to $0.57 ; \mathrm{P}=0.51$; Analysis 1.2). Studies were considered homogeneous $\left(\mathrm{I}^{2}=0 \%\right)$.

Combination of exercise modes versus walking exercise Two studies reported PFWD after 12 weeks of training, with a sample size of 63 participants (Kropielnicka 2018; Treat-Jacobson 2009). Little to no difference was found between groups (SMD $-0.22,95 \% \mathrm{Cl}-0.74$ to $0.30 ; \mathrm{P}=0.40 ;$ Analysis 1.2$)$. Included studies were considered homogeneous $\left(1^{2}=0 \%\right)$.

Arm ergometry versus walking exercise Only one small study investigated arm ergometry compared to walking exercise and no clear differences were detected in PFWD between the groups (SMD $-0.65,95 \% \mathrm{Cl}-1.66$ to 0.36 ; 18 participants; Analysis 1.2) (Treat-Jacobson 2009). 


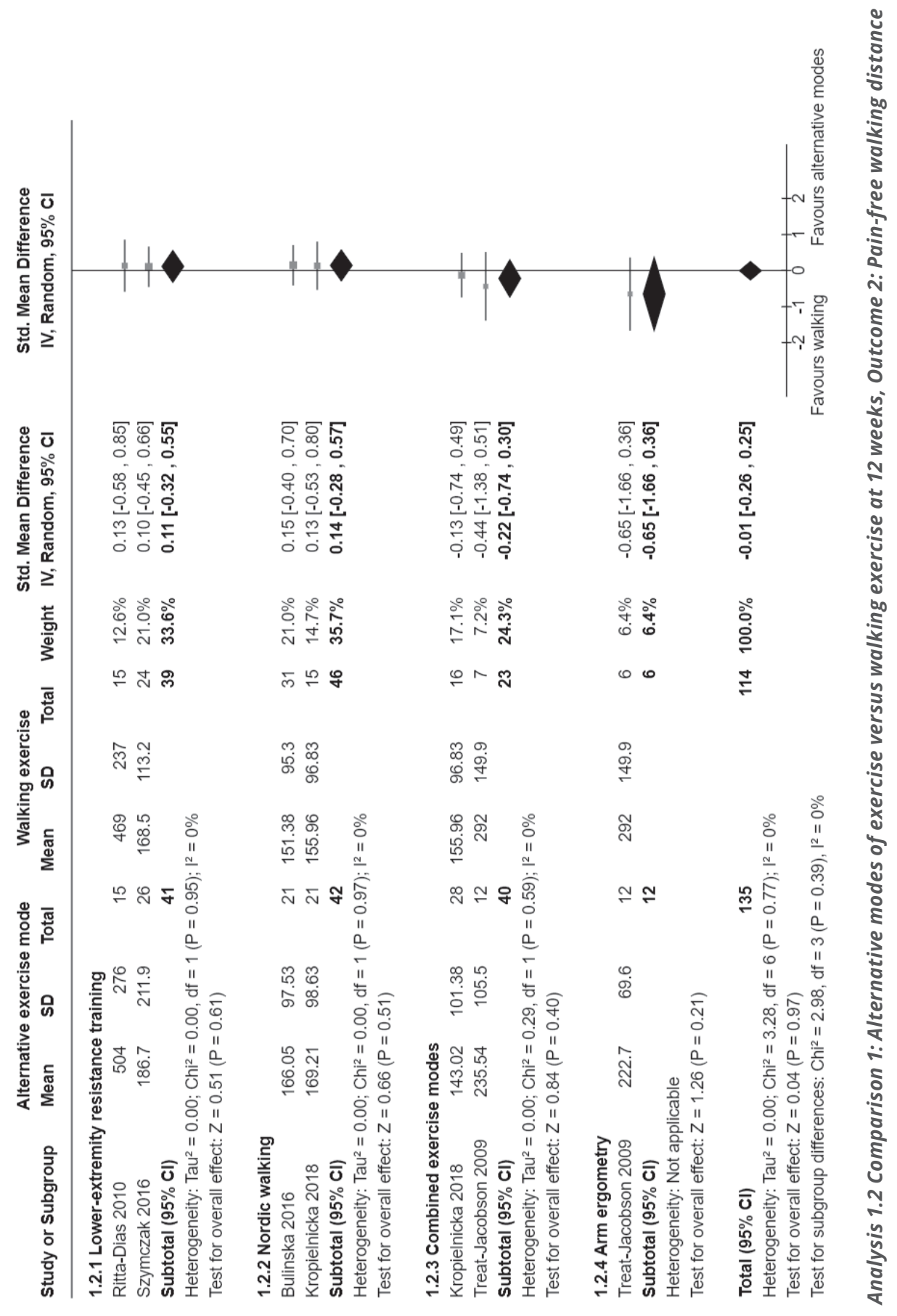




\section{Alternative modes of exercise training compared to traditional walking exercise at end of training}

\section{Maximum walking distance (MWD)}

Nine studies reported the MWD at the end of training with a total sample size of 412 participants (Bulinska 2016; Collins 2012; Kropielnicka 2018; McDermott 2009; Regensteiner 1996; Ritta-Dias 2010; Sanderson 2006; Szymczak 2016; TreatJacobson 2009). The duration of exercise programmes varied from 6 to 24weeks. Pooling detected no clear difference between groups (SMD $-0.11,95 \% \mathrm{Cl}-0.33$ to $0.11 ; P=0.32$; low-certainty evidence, see Analysis 2.1). The impact of heterogeneity was considered to be low $\left(P=0.30 ; I^{2}=15 \%\right)$. Sensitivity analysis was carried out by removing studies at risk of bias (Bulinska 2016; Kropielnicka 2018; Szymczak 2016), and resulted in a small to moderate effect on MWD in favour of walking exercise, with a SMD of $-0.36\left(95 \% \mathrm{Cl}-0.63\right.$ to $\left.-0.10 ; \mathrm{P}=0.008 ; \mathrm{I}^{2}=0 \%\right)$ (Figure 4). 


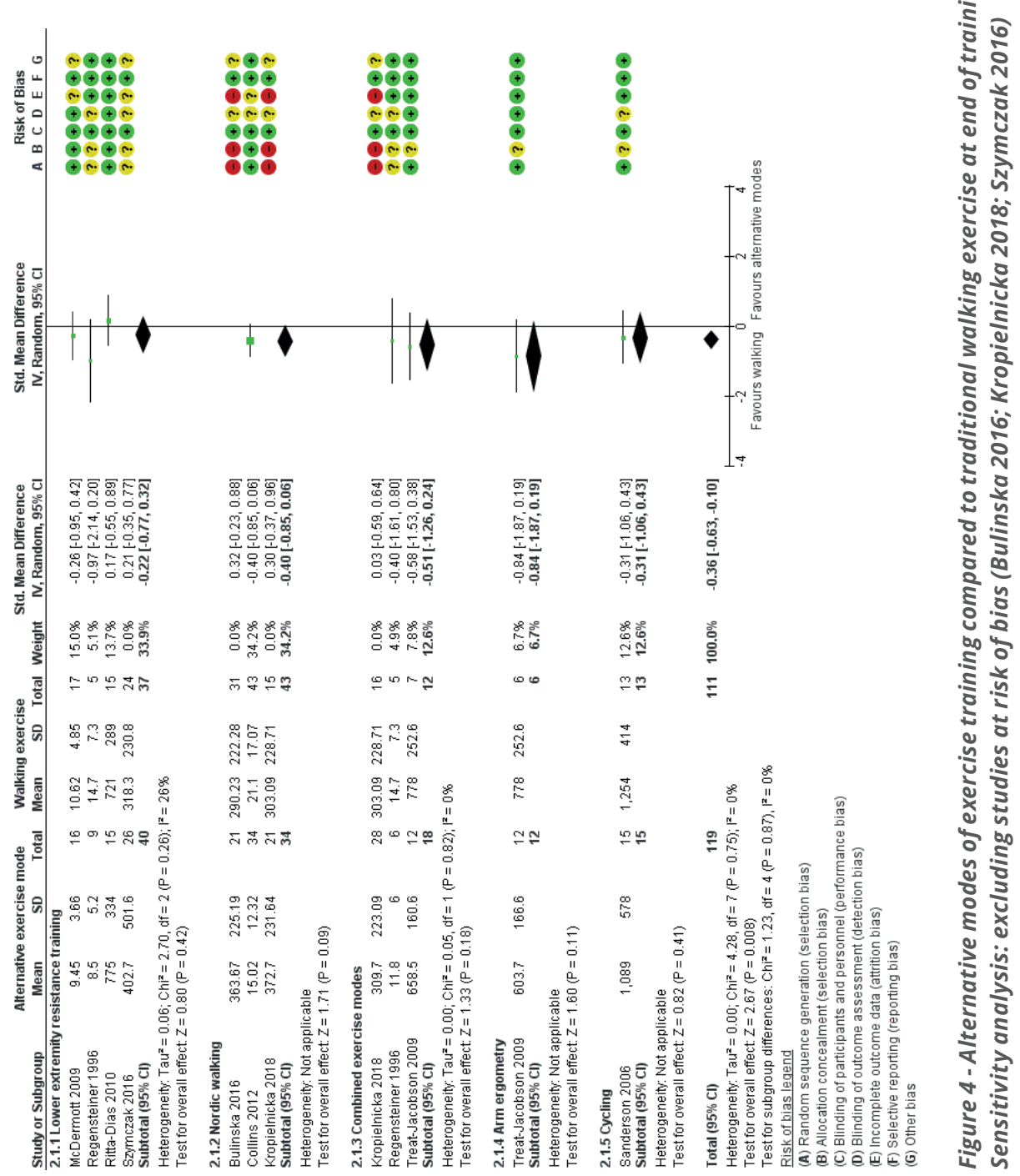


We investigated different modes of exercise by subgroup analysis (see Analysis 2.1). These are summarised below. No clear differences were detected by the test for subgroup differences $(P=0.62)$.

Lower extremity resistance training versus walking exercise Four studies reported the MWD at the end of training with a sample size of 127 participants (McDermott 2009; Regensteiner 1996; Ritta-Dias 2010; Szymczak 2016). The duration of exercise programmes varied from 12 to 24 weeks. There was no clear difference in MWD(SMD-0.06, 95\% Cl-0.48 to 0.35; $\mathrm{P}=0.77$; Analysis 2.1). Heterogeneity was considered low $\left(P=0.27 ; I^{2}=24 \%\right)$ and sensitivity analysis had no significant effect on the SMD.

Nordic walking versus walking exercise Three studies reported MWD at the end of training, with a sample size of 165 participants and programme duration varying from 12 to 24 weeks (Bulinska 2016; Collins 2012; Kropielnicka 2018). No clear difference was found (SMD 0.04, 95\% Cl -0.47 to 0.54; $\mathrm{P}=0.88$; Analysis 2.1). The moderate heterogeneity $\left(P=0.08, l^{2}=60 \%\right)$ seemed largely due to one study (Collins 2012). Sensitivity analysis to exclude this trial did result in statistical homogeneity, but did not significantly alter the SMD.

Combination of exercise modes versus walking exercise Three studies compared combination of exercise modes with walking exercise (Kropielnicka 2018; Regensteiner 1996; Treat-Jacobson 2009). As all three studies had a training duration of 12 weeks, results are reported above (Analysis 1.1).

Arm ergometry versus walking exercise Only Treat-Jacobson 2009 compared arm ergometry with walking exercise. As the training duration was 12 weeks, results are reported above (Analysis 1.1).

Cycling versus walking exercise Only one small study with 28 participants compared cycling to walking exercise and no differences were detected in MWD between the groups (SMD $-0.31,95 \% \mathrm{Cl}-1.06$ to $0.43 ; P=0.41$; Analysis 2.1) (Sanderson 2006). 


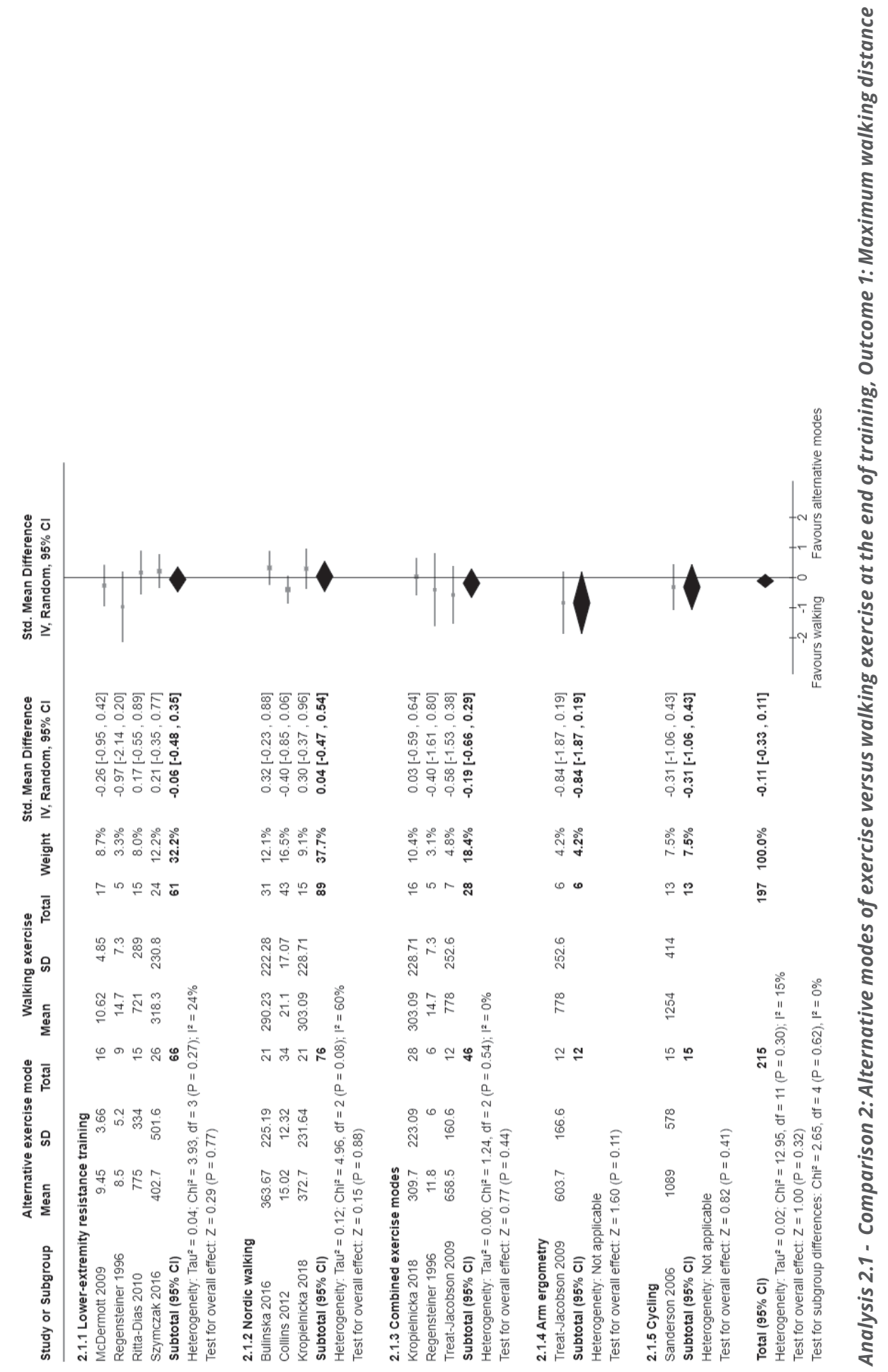




\section{Pain-free walking distance (PFWD)}

Eight studies reported the PFWD at the end of training with a sample size of 382 participants (Bulinska 2016; Collins 2012; Kropielnicka 2018; McDermott 2009; RittaDias 2010; Sanderson 2006; Szymczak 2016; Treat-Jacobson 2009). The duration of exercise programmes varied from 6 to 24 weeks. There was no clear difference between groups with a small SMD of $-0.06(95 \% \mathrm{Cl}-0.30$ to 0.17 ; $\mathrm{P}=0.59$; lowcertainty evidence, see Analysis 2.2). The heterogeneity of included studies was considered low $\left(P=0.24, I^{2}=22 \%\right)$. Sensitivity analysis by removing studies with a risk of bias did not alter the SMD (Bulinska 2016; Kropielnicka 2018; Szymczak 2016).

We investigated different modes of exercise by subgroup analysis (see Analysis 2.2). These are summarised below. The test for subgroup differences detected a difference $\left(P=0.04, I^{2}=60.4 \%\right)$, which seemed to be caused by the cycling subgroup that included only one study (Sanderson 2006). After removing this study, no subgroup differences were detected $\left(P=0.27, I^{2}=23.5 \%\right)$.

Lower extremity resistance training versus walking exercise Three studies reported PFWD at the end of training, with a sample size of 108 participants (McDermott 2009; Ritta-Dias 2010; Szymczak 2016). The duration of exercise programmes varied from 12 to 24 weeks. There was no clear difference between groups (SMD 0.01; 95\% Cl -0.37 to 0.38; P = 0.98; Analysis 2.2). The included studies were considered homogeneous $\left(I^{2}=0 \%\right)$ and sensitivity analyses had no important effect on the SMD.

Nordic walking versus walking exercise Three studies reported PFWD at the end of training, with a sample size of 165 participants and programme duration varying from 12 to 24 weeks (Bulinska 2016; Collins 2012; Kropielnicka 2018). The SMD of 0.21 represented little to no difference between groups $(95 \% \mathrm{Cl}-0.10$ to $0.52 ; \mathrm{P}=0.19)$. Studies were considered homogeneous $\left(I^{2}=0 \%\right)$.

Combination of exercise modes versus walking exercise Two studies compared combination of exercise modes with walking exercise (Kropielnicka 2018; TreatJacobson 2009). As these had a training duration of 12 weeks, results are reported above (Analysis 1.2).

Arm ergometry versus walking exercise Only Treat-Jacobson 2009 compared arm ergometry with walking exercise. As the training duration was 12 weeks, results are reported above (Analysis 1.2).

Cycling versus walking exercise Only one small study with 28 participants investigated cycling compared to walking exercise and a possible benefit from walking exercise may be seen in PFWD between the groups (SMD $-1.01,95 \% \mathrm{CI}$ -1.81 to $-0.22 ; \mathrm{P}=0.01$; Analysis 2.2) (Sanderson 2006). 


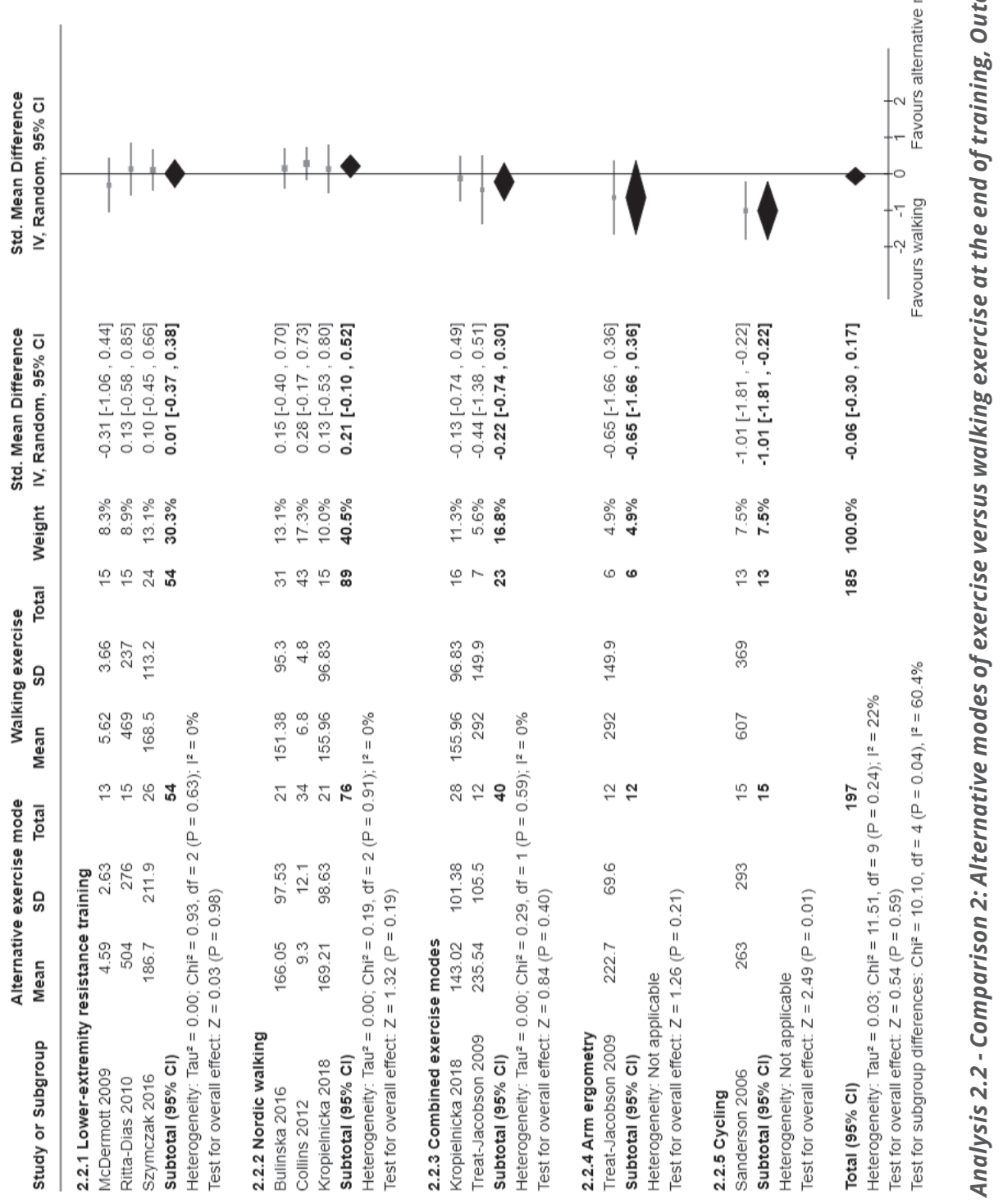




\section{Health-related quality of life (HR-QoL)}

Four studies described HR-QoL (Collins 2012; Delaney 2015; McDermott 2009; Regensteiner 1996). Two studies reported the physical functioning score of the SF-36 (Collins 2012; McDermott 2009). Because of the skewed data of one of the studies (McDermott 2009), with relatively small sample size, we did not perform a meta-analysis. Both studies found an improvement in both exercise groups. In McDermott 2009, the physical functioning score improved to a median score of 50.0 (interquartile range 40.0 to 60.0 ) after 24 weeks of treadmill walking ( $n=17$ ) and to a median score of 60.0 (interquartile range 35.0 to 70.0) after strength training ( $n=14$, $P=0.811$ ). In Collins 2012, the mean score was 60.42 (SD 21.75) after 24 weeks of treadmill walking $(n=43)$ and 55.51 (SD 21.27) after Nordic walking $(n=34, P=0.22)$.

Regensteiner 1996, with a sample size of 25 participants, used the Medical Outcomes Study (MOS) SF-20, which consists of five domain scores: physical functioning score, social functioning score, role functioning score, overall health score, and well-being score. After 12 weeks of training, the physical functioning scores improved by $24 \%$ for the walking group ( $95 \%$ Cl 9 to 39), and the well-being scores increased by $9 \%$ ( $95 \% \mathrm{Cl} 2$ to 16$)$ for the lower-extremity resistance training group. No improvement in the other MOS domains was detected. In the combined group, no improvement in MOS scores was seen.

Delaney 2015 used the Australian Vascular Quality of Life Index, in which a lower score represents higher quality of life. It found an improved HR-QoL in both the treadmill walking group $(n=15)$ and the combination exercise group $(n=12)$, with a mean decrease of 1.6 points (SD 2.4) and 3.3 points (SD 3.9), respectively $(P=0.18)$.

\section{Self-reported functional impairment}

Three studies used the WIQ to measure functional impairment (Collins 2012; McDermott 2009; Regensteiner 1996). All three studies reported the WIQ distance score and two studies also reported the speed and stair-climbing scores (McDermott 2009; Regensteiner 1996). One study had relatively small sample size and a nonnormal distribution of outcomes (McDermott 2009). Therefore, it was considered inappropriate to transform these skewed data to enable pooling. Meta-analysis of the two remaining studies, with a sample size of 96 participants, showed little or no difference between the study arms, with a mean difference of -5.52 ( $95 \% \mathrm{Cl}-17.41$ to $6.36, P=0.36$; low-certainty evidence; see Analysis 2.3 ). The included studies were considered homogeneous $\left(I^{2}=0 \%\right)$. 


\begin{tabular}{|c|c|c|c|c|c|c|c|c|c|}
\hline \multirow[b]{2}{*}{ Study or Subgroup } & \multicolumn{3}{|c|}{ Alternative exercise mode } & \multicolumn{3}{|c|}{ Walking exercise } & \multirow[b]{2}{*}{ Weight } & \multirow{2}{*}{$\begin{array}{c}\text { Mean Difference } \\
\text { IV, Random, } 95 \% \mathrm{Cl}\end{array}$} & \multirow{2}{*}{$\begin{array}{c}\text { Mean Difference } \\
\text { IV, Random, } 95 \% \mathrm{Cl}\end{array}$} \\
\hline & Mean & SD & Total & Mean & SD & Total & & & \\
\hline \multicolumn{10}{|c|}{ 2.3.1 Lower-extremity resistance training } \\
\hline Regensteiner 1996 & 42.5 & 22.6 & 9 & 56.5 & 27.7 & 10 & $27.6 \%$ & $-14.00[-36.64,8.64]$ & \\
\hline Subtotal $(95 \% \mathrm{Cl})$ & & & 9 & & & 10 & $27.6 \%$ & $-14.00[-36.64,8.64]$ & \\
\hline \multicolumn{10}{|c|}{ Heterogeneity: Not applicable } \\
\hline \multicolumn{10}{|c|}{ Test for overall effect: $Z=1.21(P=0.23)$} \\
\hline \multicolumn{10}{|l|}{ 2.3.2 Nordic walking } \\
\hline Collins 2012 & 45.5 & 31.7 & 34 & 47.8 & 30.2 & 43 & $72.4 \%$ & $-2.30[-16.26,11.66]$ & \\
\hline Subtotal $(95 \% \mathrm{Cl})$ & & & 34 & & & 43 & $72.4 \%$ & $-2.30[-16.26,11.66]$ & \\
\hline \multicolumn{10}{|c|}{ Heterogeneity: Not applicable } \\
\hline \multicolumn{10}{|c|}{ Test for overall effect: $Z=0.32(P=0.75)$} \\
\hline Total $(95 \% \mathrm{Cl})$ & & & 43 & & & 53 & $100.0 \%$ & $-5.52[-17.41,6.36]$ & \\
\hline \multicolumn{10}{|c|}{ Heterogeneity: $\operatorname{Tau}^{2}=0.00 ; \mathrm{Chi}^{2}=0.74, \mathrm{df}=1(\mathrm{P}=0.39) ; \mathrm{I}^{2}=0 \%$} \\
\hline \multicolumn{9}{|c|}{ Test for overall effect: $Z=0.91(P=0.36)$} & $-100-50$ \\
\hline
\end{tabular}

Analysis 2.3 - Comparison 2: Alternative modes of exercise versus walking exercise at the end of training, Outcome 3: Functional impairment (WIQ distance score)

In McDermott 2009, the WIQ distance score improved in both the strength $(n=15)$ and treadmill walking group $(n=15)$, with a median of, respectively, 14.0 points (interquartile range $=1.56$ to 26.6 ) and 7.46 points (interquartile range $=-0.36$ to $25.0 ; P=0.431)$. WIQ speed score improved in both the strength $(n=15)$ and treadmill walking group $(n=16)$, with a median of, respectively, 3.26 points (interquartile range $=-7.61$ to 26.1 ) and 1.63 points (interquartile range $=-3.80$ to 28.8; $\mathrm{P}=0.736)$. WIQ stair-climbing score improved in the strength training group $(n=15)$, with a median of, respectively, 12.5 points (interquartile range $=4.17$ to 25.0 ), while no improvement was seen in the median score in the treadmill walking group ( $n=16$, median score of 0.00 points, interquartile range $=0.00$ to $14.6 ; P=0.136$ ).

Finally, the remaining study (Regensteiner 1996), showed little or no difference in the WIQ speed score with an effect size of $-4.50 \%(95 \% \mathrm{CI}-27.34$ to $18.34 ; \mathrm{P}=0.70)$ and a decreased WIQ stair-climbing score with an effect size of $-29.50 \%(95 \% \mathrm{Cl}$ -51.65 to $-7.35, P=0.009$ ), both in favour of alternative exercise. 


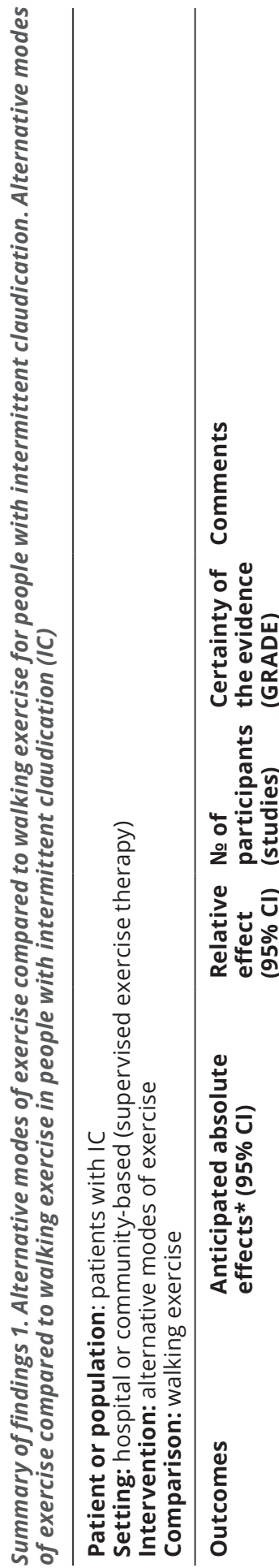

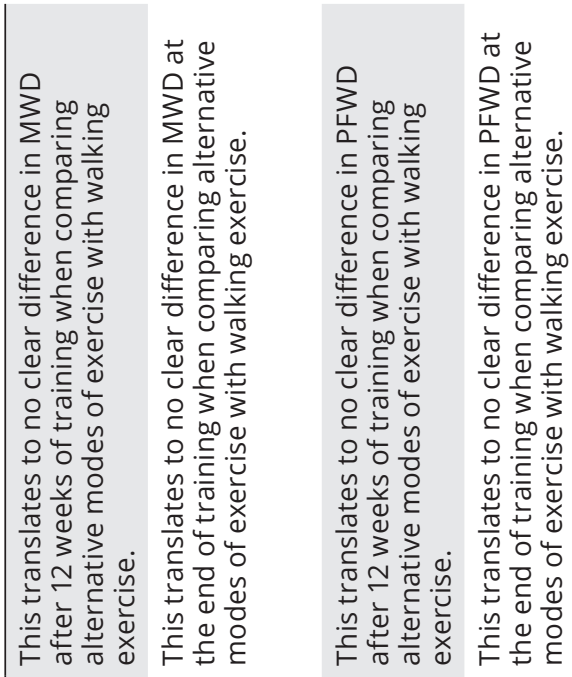

\begin{tabular}{|c|c|c|}
\hline 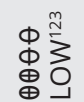 & 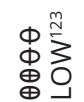 & 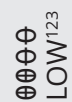 \\
\hline 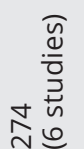 & 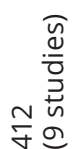 & 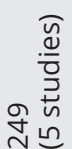 \\
\hline
\end{tabular}
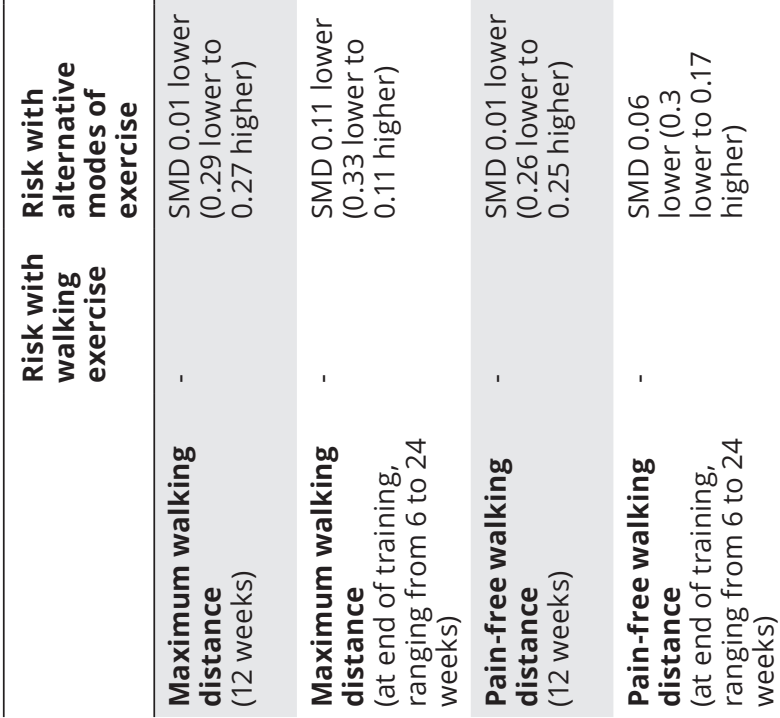


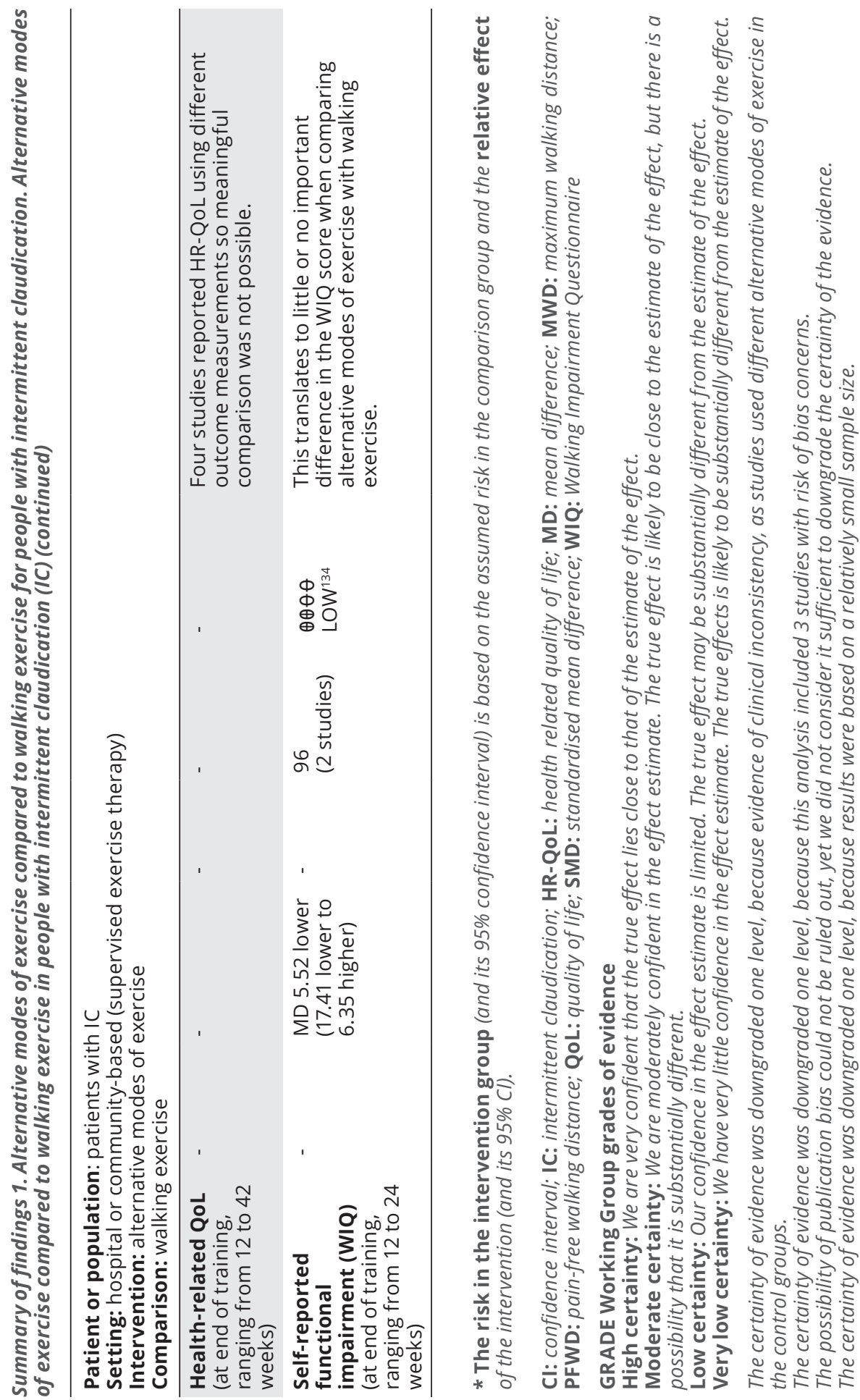




\section{DISCUSSION}

\section{Summary of main results}

See Summary of findings 1. In this update, five additional studies were included, making a total of 10 studies that randomised a total of 527 participants. The alternative modes of exercise therapy included cycling, lower-extremity resistance training, upper-arm ergometry, Nordic walking, and combinations of exercise modes. Besides randomised controlled trials, two quasi-randomised trials were included. Our assessment of the overall risk of bias of included studies varied from high to low risk of bias. Using the GRADE criteria, the certainty of evidence was judged to be low due to the relatively small sample sizes, clinical inconsistency, and inclusion of three studies with risk of bias concerns.

Overall, no clear differences were detected between alternative exercise modes and walking exercise for mean walking distance (MWD) at 12 weeks (standardised mean difference (SMD) $-0.01,95 \% \mathrm{Cl}-0.29$ to $0.27 ; \mathrm{P}=0.95 ; 6$ studies; 274 participants; low-certainty evidence); or at the end of training (SMD $-0.11,95 \% \mathrm{CI}$ -0.33 to $0.11 ; \mathrm{P}=0.32 ; 9$ studies; 412 participants; low-certainty evidence). Similarly, no clear differences were detected for PFWD at 12 weeks (SMD-0.01, 95\% Cl-0.26 to $0.25 ; \mathrm{P}=0.97 ; 5$ studies; 249 participants; low-certainty evidence) or at the end of training (SMD $-0.06,95 \% \mathrm{Cl}-0.30$ to $0.17 ; \mathrm{P}=0.59 ; 8$ studies, 382 participants; low-certainty evidence).

Four studies reported on HR-QoL and three studies reported on functional impairment. However, meta-analysis was only possible for the WIQ distance score, which demonstrated little or no important difference between study groups (MD -5.52, 95\% Cl -17.41 to 6.36; $\mathrm{P}=0.36 ; 2$ studies; 96 participants; low-certainty evidence).

We carried out subgroup analyses by type of alternative exercise mode (cycling, lower-extremity resistance training, upper-arm ergometry, Nordic walking, and combinations of exercise modes) for the main outcomes of MWD and PFWD at 12 weeks and end of training. No differences were detected by the test for subgroup differences in MWD or PFWD at 12 weeks $(P=0.16 ; P=0.39$; respectively). At the end of training, no difference was detected between the subgroups for MWD $(P=0.62)$; but a difference was detected in PFWD $(P=0.04)$, which seemed to be caused by the cycling subgroup that included only one study with 28 participants (Sanderson 2006). After removing this study, no subgroup differences were detected $(P=0.27$, $\left.I^{2}=23.5 \%\right)$.

Sensitivity analysis did not significantly alter the results, except for the primary comparison of MWD at the end of training. In this comparison, excluding studies with apparent risk of bias resulted in a small to moderate effect on MWD in favour of walking exercise

(SMD - $0.36,95 \% \mathrm{Cl}-0.63$ to $-0.10 ; \mathrm{P}=0.0008$; 5 studies; 230 participants; lowcertainty evidence). This might suggest that, in the absence of the influence of 
bias, walking exercise interventions provide more benefit for MWD at the end of intervention compared to alternative modes of exercise. The use of SMDs makes it difficult to determine whether the difference was clinically relevant. Interpretation is further complicated by the fact that the alternative exercise group includes different types of exercises. Moreover, our level of certainty regarding this evidence is low considering it was from a small number of studies with relatively small sample size. Future research is likely to have a relevant impact on our confidence in the estimate of effect.

\section{Overall completeness and applicability of evidence}

Although the subject of this review is increasing in interest, only five new studies could be included since the previous search of July 2013. In addition to this, we have identified some possible factors which may limit the completeness and applicability of the evidence presented:

\section{Participants}

The enrolment criteria were relatively homogeneous among included studies. All studies excluded participants if the exercise capacity was limited by comorbidity. However, comorbidity is common among patients with IC, as they are mostly elderly. Therefore, excluding participants with comorbidity probably affects the generalisability of the observed effects.

\section{Interventions}

The vast majority of studies met the guidelines' recommendations for a minimum programme duration of 12 weeks, with durations ranging from six weeks (Sanderson 2006), to 12 weeks (Bulinska 2016; Delaney 2015; Kropielnicka 2018; Regensteiner 1996; Ritta-Dias 2010; Szymczak 2016; Treat-Jacobson 2009), and 24 weeks (Collins 2012, McDermott 2009). The included studies reported outcomes on five alternative modes of exercise, namely cycling (Sanderson 2006), lower-extremity resistance training (McDermott 2009; Regensteiner 1996; Ritta-Dias 2010; Szymczak 2016), upperarm ergometry (Treat-Jacobson 2009), Nordic walking (Bulinska 2016; Collins 2012; Kropielnicka 2018), and combinations of exercise modes (Kropielnicka 2018; Regensteiner 1996; Treat-Jacobson 2009). Subgroup analysis was carried out with the available data but each subgroup included a small number of studies. Overall, more studies are needed to make meaningful comparisons between each alternative exercise mode and the current standard of walking exercise.

\section{Outcomes}

Only a few studies reported HR-QoL (Collins 2012; Delaney 2015; McDermott 2009; Regensteiner 1996), using different measuring instruments, and self-reported functional impairment (Collins 2012; McDermott 2009; Regensteiner 1996). Meta- 
analysis was only possible for one of the WIQ sub scores. Therefore, more studies are needed to make meaningful comparisons for these outcomes.

\section{Applicability}

For the overall meta-analysis, the sample size seemed sufficient, but the results should be interpreted with caution because of the risk of bias ranging from high to low and heterogeneity due to the different types of alternative interventions included and the different units of outcomes across studies. Participant numbers for the subgroup analyses were limited. The SMDs were not back-transformed to metres or seconds, because this could be misleading and there is no consensus in the literature on a minimum clinically relevant difference in metres. Using the rules of thumb for effect sizes might simplify interpretation of the results (SMD of 0.2 represents a small effect, 0.5 a moderate effect, and 0.8 a large effect).

The approach chosen in this review has two main limitations that need to be considered. Different types of interventions were lumped together given that our primary aim was to compare alternative modes of exercise to traditional treadmill walking, and the small number of studies looking at different modes of exercises complicated sub analysis. Furthermore, a non-inferiority approach would have been ideal, but this was not feasible due to the mixed alternative modes of exercise group and the impossibility of predetermining a substantiated non-inferiority margin. Despite these limitations, this updated review provides valuable information.

This review focused on treadmill walking distances. However, treadmill walking does not necessarily correlate to walking ability in daily life (Gommans 2016), nor does an increased treadmill walking distance always lead to more walking (Fokkenrood 2015). Additionally, a possible training effect of treadmill walking might affect treadmill testing, which probably causes an underestimation of the effect of alternative exercise modes. Because of these aspects, focusing on treadmill walking capacity alone probably has limited value in optimising the treatment of IC. Moreover, improving physical fitness and reducing cardiovascular risk, e.g. by increasing physical activity, are important treatment goals in IC. Therefore, objective measurements of functional impairment, physical activity, and physical condition might be interesting, provided that they are measured with validated measuring instruments. Unfortunately, the current lack of consensus and availability of reliable instruments leads to a high heterogeneity with regard to the use of measuring instruments and outcomes.

\section{Quality of the evidence}

The risk of bias of included studies varied from high to low and three studies were considered to have risk of bias concerns (Bulinska 2016; Kropielnicka 2018; Szymczak 2016) (see Figure 2 and Figure 3). This review demonstrates that alternative modes of exercise therapy seem to yield similar results to walking therapy and may be considered useful when supervised walking exercise is not an option for the patient. 
The certainty of the evidence according to the GRADE criteria was low for the overall comparison of alternative exercise modes versus walking exercise (Summary of findings 1). The certainty was downgraded due to the relatively small sample sizes, clinical inconsistency, and concerns over risk of bias. Furthermore, we could not investigate possible publication bias, because the limited number of studies did not allow for assessment of asymmetry in funnel plots.

\section{Potential biases in the review process}

We used the SMD as a summary statistic to reduce potential heterogeneity due to the use of different treadmill protocols between studies. Furthermore, we performed subgroup analyses if data were available. It was not possible to conduct a formal analysis of possible publication bias by using a funnel plot owing to the limited number of studies included in the meta-analysis. Several studies were excluded, solely because they did not report the correct outcome measures. Although we carefully ascertained that relevant outcomes were not available because they were not measured rather than not reported, this could introduce bias in the review process. In future updates, we will pay further attention to this potential source of bias.

Many studies were excluded in the title-abstract screening, because they were not RCTs, examined non supervised programmes or compared alternative modes of exercise to a non-exercise control group. Furthermore, this review only included supervised exercise programmes, thus excluding home-based or partially supervised programmes comparing walking exercise to alternative modes of exercise. However, considering the findings of other studies, this did not substantially alter results (see Agreements and disagreements with other studies or reviews).

\section{Agreements and disagreements with other studies or reviews}

The findings of this update are in line with the findings of the previous version of this review (Lauret 2014), as well as findings of several other systematic reviews. One previously published systematic review (Parmenter 2011), which included unsupervised exercise programmes and RCTs with a non-exercise control group, found no clear difference between alternative aerobic exercise modes and walking exercise for IC. The data on resistance training were considered insufficient to make clinical recommendations and further research was needed. A systematic review and meta-analysis on Nordic walking (Golledge 2018) suggested that Nordic walking might be more effective in improving cardiovascular fitness than standard walking. However, no benefit of Nordic walking was found compared to standard walking, including unsupervised and supervised settings. A subgroup analysis of completely supervised programmes also demonstrated similar improvements in MWD (SMD $-0.79 ; 95 \% \mathrm{Cl}-2.81$ to $1.24 ; \mathrm{P}=0.446)$. Furthermore, a recent statement from the American Heart Association (Treat-Jacobson 2019), was based on a systematic review, which included the comparison of treatment modalities. Evidence for each 
of the alternative exercise modes was summarised separately, and the authors concluded that modalities other than supervised treadmill exercise, including arm ergometry, cycling, and possibly resistance training, can also improve relevant outcomes. In summary, our findings agree with currently available evidence that alternative exercise modes are as effective as standard walking, however, more evidence is needed.

\section{AUTHORS' CONCLUSIONS}

\section{Implications for practice}

Overall, this review demonstrates that there is no clear difference between alternative modes of exercise and supervised walking exercise regarding maximum and pain-free walking distance in patients with intermittent claudication, which means that both interventions yield similar improvements. The certainty of this evidence was judged to be low, mainly due to the small sample size and risk of bias concerns. The findings of this review indicate that alternative exercise modes may be useful when supervised walking exercise is not an option. This is consistent with current international guidelines (Aboyans 2018; Gerhard-Herman 2017).

\section{Implications for research}

Although this update included five additional studies, the overall sample size was relatively small. More RCTs with adequate methodological quality and sufficient power are needed to provide more robust evidence for comparisons between each alternative exercise mode and the current standard of supervised treadmill walking. Those RCTs should preferably investigate exercise programmes with a minimum of three sessions a week for a consecutive 12 weeks, as this is recommended based on currently available evidence (Treat-Jacobson 2019). Walking capacity should not solely be measured with treadmill tests, as this might result in an underestimation of the effect of alternative exercise modes and probably does not represent 'daily life' walking. Future studies should investigate functional outcome measures on walking behaviour, physical activity, cardiovascular risk, and HR-QoL, using standardised testing methods and reporting of outcomes to allow meaningful comparison across studies. Furthermore, studies should include patients with comorbidity in order to be more representative of the PAD population. Finally, research is needed to explore possibilities to optimise exercise programmes, taking into account the personal preferences, therapy compliance, and long-term effects. It is thought that more varied and personalised programmes will possibly improve therapy adherence. Furthermore, this might lead to better compliance with the recommendations for physical activity, because combinations of aerobic and muscle-strengthening activities are preferable, according to the Physical Activity Guidelines (Piercy 2018). 


\section{ACKNOWLEDGEMENTS}

We acknowledge the support provided by Dr Marlene Stewart, Managing Editor of Cochrane Vascular, Dr Cathryn Broderick, Assistant Managing Editor of Cochrane Vascular, and Ms Candida Fenton, Cochrane Vascular Information Specialist, in updating this review. We would also like to thank the previous authors for their contributions to the previous version of this review: Professor Hunink and Dr Spronk. The review authors, and the Cochrane Vascular editorial base, wish to thank the following peer reviewers for their input as well as the reviewer who opted to remain anonymous: Dr Ralph G DePalma, Uniformed Services University of the Health Sciences, Washington and Department of Veteran Affairs, Washington, US; Associate Professor Anthony Leicht, James Cook University, Australia; Dr Jonathan Moran, Trinity College Dublin, Ireland; LeAnne Lovett-Floom, DNP, MSN, RN, PHNBC, US. 


\section{REFERENCES}

\section{References to studies included in this review}

Bulinska 2016 \{published data only\} Bulinska K, Kropielnicka K, Jasinski T, WojcieszczykLatos J, Pilch U, Dabrowska G, et al. Nordic pole walking improves walking capacity in patients with intermittent claudication: a randomized controlled trial. Disability and Rehabilitation 2016;38(13):1318-24. [PMID: 26305413]

Collins 2012 \{published data only\}

Collins EG, McBurney C, Butler J, Jelinek C, O'Connell S, Fritschi C, et al. The effects of walking or walking-withpoles training on tissue oxygenation in patients with peripheral arterial disease. International Journal of Vascular Medicine 2012;2012:985025. [PMID: 23050152]

* Collins EG, O'Connell S, McBurney C, Jelinek C, Butler J, Reda D, et al. Comparison of walking with poles and traditional walking for peripheral arterial disease rehabilitation. Journal of Cardiopulmonary Rehabilitation and Prevention 2012;32(4):210-8. [PMID: 22595894]

Delaney 2015 \{published data only\} Delaney $C L$, Miller MD, Allan RB, Spark JI. The impact of different supervised exercise regimens on endothelial function in patients with intermittent claudication. Vascular 2015;23(6):561-9. [PMID: 25406267]

Kropielnicka 2018 \{published data only\} Kropielnicka K, Dziubek W, Bulinska K, Stefanska M, Wojcieszczyk-Latos J, Jasinski $R$, et al. Influence of the physical training on muscle function and walking distance in symptomatic peripheral arterial disease in elderly. BioMed Research International 2018;2018:1937527. [PMID: 30345295] McDermott 2009 \{published data only\}
* McDermott MM, Ades P, Guralnik JM, Dyer A, Ferrucci L, Liu K, et al. Treadmill exercise and resistance training in patients with peripheral arterial disease with and without intermittent claudication: a randomized controlled trial. Journal of the American Medical Association 2009;301(2):165-74. [PMID: 19141764]

McDermott MM, Kibbe MR, Guralnik JM, Ferrucci L, Criqui MH, Domanchuk $K$, et al. Durability of benefits from supervised treadmill exercise in people with peripheral artery disease. Journal of the American Heart Association 2019;8(1):e009380. [PMID: 30587066]

Regensteiner 1996 \{published data only\}

Hiatt WR, Regensteiner JG, Wolfel EE, Carry MR, Brass EP. Effect of exercise training on skeletal muscle histology and metabolism in peripheral arterial disease. Journal of Applied Physiology 1996;81(2):780-8. [PMID: 8872646]

Hiatt WR, Wolfel EE, Meier RH, Regensteiner JG. Superiority of treadmill walking exercise versus strength training for patients with peripheral arterial disease. Implications for the mechanism of the training response. Circulation 1994;90(4):1866-74. [PMID: 7923674]

*Regensteiner JG, Steiner JF, Hiatt WR. Exercise training improves functional status in patients with peripheral arterial disease. Journal of Vascular Surgery 1996;23(1):104-15. [PMID: 8558725]

Ritta-Dias 2010 \{published data only\}

Grizzo Cucato G, De Moraes Forjaz CL, Kanegusuku H, Da Rocha Chechuen M, Riani Costa LA, Wolosker N, et al. Effects of walking and strength training on resting and exercise cardiovascular responses in patients with intermittent claudication. Vasa 2011;40(5):390-7. [PMID: 21948782] 
Menêses $A L$, De Lima $G H$, Forjaz $C L$, Lima $A H$, Silva GQ, Cucato GG, et al. Impact of a supervised strength training or walking training over a subsequent unsupervised therapy period on walking capacity in patients with claudication. Journal of Vascular Nursing 2011;29(2):81-6. [PMID: 21558030]

* Ritti-Dias RM, Wolosker N, De Moraes Forjaz $\mathrm{CL}$, Carvalho CR, Cucato GG, Leão PP, et al. Strength training increases walking tolerance in intermittent claudication patients: randomized trial. Journal of Vascular Surgery 2010;51(1):89-95. [PMID: 19837534]

Sanderson 2006 \{published data only\} Sanderson B, Askew C, Stewart I, Walker $P$, Gibbs H, Green S. Short-term effects of cycle and treadmill training on exercise tolerance in peripheral arterial disease. Journal of Vascular Surgery 2006;44(1):119-27. [PMID: 16828435]

Szymczak 2016 \{published data only\} Szymczak M, Oszkinis G, Majchrzycki $M$. The impact of walking exercises and resistance training upon the walking distance in patients with chronic lower limb ischaemia. BioMed Research International 2016;2016:7515238. [PMID: 27833919]

Treat-Jacobson 2009 \{published data only\}

Bronas UG, Treat-Jacobson D, Leon AS. Comparison of the effect of upper body ergometry aerobic training vs treadmill training on central cardiorespiratory improvement and walking distance in patients with claudication. Journal of Vascular Surgery 2011;53(6):1557-64. [PMID: 21515017]

* Treat-Jacobson D, Bronas UG, Leon AS. Efficacy of arm-ergometry versus treadmill exercise training to improve walking distance in patients with claudication. Vascular Medicine 2009;14(3):203-13. [PMID: 19651669]

\section{References to studies excluded from this review}

Dedes 2010 \{published data only\} Dedes H, Figoni SF, Kalioundji G, Kunkel C, Peter $A$, Phillips $A$, et al. Prospective trial of calf ergometry training on walking ability in peripheral arterial disease. PM \& R: Journal of Injury, Function, and Rehabilitation 2010;2(9 Suppl 1):S26.

Delaney 2014 \{published data only\}

* Delaney CL, Miller MD, Chataway TK, Spark JI. A randomised controlled trial of supervised exercise regimens and their impact on walking performance, skeletal muscle mass and calpain activity in patients with intermittent claudication. European Journal of Vascular and Endovascular Surgery 2014;47(3):304-10. [PMID: 24445084]

Delaney CL, Spark JI. A randomised controlled trial of two supervised exercise regimens and their impact on inflammatory burden in patients with intermittent claudication. Vascular 2016;24(3):264-72. [PMID: 26567275]

EXERT Study 2018 \{published data only\}

Kirk LN, Brown R, Treat-Jacobson D. Longterm outcomes of supervised exercise in peripheral artery disease: impact of differing modes of exercise 1-4 years after intervention. Journal of Vascular Nursing 2018;36(3):121-8. [PMID: 30139449]

* NCT00895635. Evaluating two exercise training programs to reduce leg pain in people with peripheral arterial disease (the EXERT study). clinicaltrials.gov/ct2/ show/NCT00895635 (first received 8 May 2009).

Gardner 2011 \{published data only\} Gardner AW. Supervised exercise therapy provided by local physiotherapists improves walking distance in patients with claudication. Evidence-based Medicine 2011;16(2):43-4. [PMID: 21427053] 
Jones 1996 \{published data only\} Jones PP, Skinner JS, Smith LK, John FM, Bryant CX. Functional improvements following Stairmaster versus treadmill exercise training for patients with intermittent claudication. Journal of Cardiopulmonary Rehabilitation 1996;16(1):47-55. [PMID: 8907442]

Kao 2012 \{published data only\} Kao K, Choi S, Etnyre G, Figoni SF, Kunkel CF, Ornelas $C C$, et al. Comparison of calf exercise and treadmill training in peripheral arterial disease. PM \& R: Journal of Injury, Function, and Rehabilitation 2012;4(10):S346.

Kim 2006 \{published data only\} Kim $\mathrm{DH}$. Exercise and peripheral arterial disease. Annals of Internal Medicine 2006;144(9):author reply 699-700. [PMID: 16670144]

Kuwabara 2010 \{published data only\} Kuwabara M, Anzai H, Nishi Y, Hayashida $\mathrm{N}$, Hisatome I. Treadmill and ergometer exercise once a week equally improve not only exercise-tolerance but also cardiorenal function in peripheral arterial disease (PAD) patients with claudication. Journal of the American College of Cardiology 2010;55(10s1):E1479-A158. [DOI: 10.1016/ S0735-1097(10)61480-5]

Nawaz 2001 \{published data only\} Nawaz S, Walker RD, Wilkinson CH, Saxton JM, Pockley AG, Wood RF. The inflammatory response to upper and lower limb exercise and the effects of exercise training in patients with claudication. Journal of Vascular Surgery 2001;33(2):392-9. [PMID: 11174795]

Oakley 2008 \{published data only\} Oakley C, Zwierska I, Tew G, Beard JD, Saxton JM. Nordic poles immediately improve walking distance in patients with intermittent claudication. European Journal of Vascular and Endovascular Surgery 2008;36(6):689-94; discussion 695-6. [PMID: 18835794]
Ornelas 2011 \{published data only\} Ornelas CC, Dhingsa K, Etnyre G, Figoni SF, Fung DA, Kunkel C, et al. Comparison of calf ergometer and treadmill exercise training interventions for participants with intermittent claudication due to peripheral arterial disease. PM \& R: Journal of Injury, Function, and Rehabilitation 2011;3(10 Suppl 1):S266-7. [DOI: 10.1016/j.pmrj.2011.08.302]

Parmenter 2013 \{published data only\} Parmenter BJ, Raymond J, Dinnen P, Lusby RJ, Fiatarone Singh MA. High-intensity progressive resistance training improves flat-ground walking in older adults with symptomatic peripheral arterial disease. Journal of the American Geriatrics Society 2013;61(11):1964-70.

Parr 2009 \{published data only\} Parr BM, Noakes TD, Derman EW. Peripheral arterial disease and intermittent claudication: efficacy of short-term upper body strength training, dynamic exercise training, and advice to exercise at home. South African Medical Journal 2009;99(11):800-4. [PMID: 20218480]

Roitman 2010 \{published data only\} Roitman JL. Treadmill exercise and resistance training in patients with peripheral arterial disease with and without intermittent claudication: a randomized controlled trial. Journal of Cardiopulmonary Rehabilitation and Prevention 2010;30(1):62.

Saxton 2008 \{published data only\} Saxton JM, Zwierska I, Hopkinson K, Espigares E, Choksy S, Nawaz S, et al. Effect of upperand lower-limb exercise training on circulating soluble adhesion molecules, hs-CRP and stress proteins in patients with intermittent claudication. European Journal of Vascular and Endovascular Surgery 2008;35(5):607-13. [PMID: 18226561] 
Saxton 2011 \{published data only\} Saxton JM, Zwierska I, Blagojevic M, Choksy SA, Nawaz S, Pockley AG. Upper- versus lower-limb aerobic exercise training on health-related quality of life in patients with symptomatic peripheral arterial disease. Journal of Vascular Surgery 2011;53(5):1265-73. [PMID: 21215558]

Tebbutt 2011 \{published data only\} Tebbutt N, Robinson L, Todhunter J, Jonker L. A plantar flexion device exercise programme for patients with peripheral arterial disease: a randomised prospective feasibility study. Physiotherapy 2011;97(3):244-9. [PMID: 21820543]

Treat-Jacobson 2011 \{published data only\} Treat-Jacobson D, Henly SJ, Bronas UG, Leon AS, Henly GA. The pain trajectory during treadmill testing in peripheral artery disease. Nursing Research 2011;60(3 Suppl):S38-49. [PMID: 21543960]

Treat-Jacobson 2012 \{published data only\} Treat-Jacobson D, Bronas UG, Krause BJ, Robinson CA, Santilli SM, Leon AS. Aerobic arm exercise training to improve outcomes for patients with severe claudication and ischemic rest pain. Vascular Medicine 2012;17(3):204.

Van Schaardenburgh 2017 \{published data only\} Van Schaardenburgh M, Wohlwend M, Rognmo O, Mattsson EJR. Exercise in claudicants increase or decrease walking ability and the response relates to mitochondrial function. Journal of Translational Medicine 2017;15(1):130. [PMID: 28592294]
Walker 2000 \{published data only\} Walker RD, Nawaz S, Wilkinson $\mathrm{CH}$, Saxton JM, Pockley AG, Wood RF. Influence of upper- and lower-limb exercise training on cardiovascular function and walking distances in patients with intermittent claudication. Journal of Vascular Surgery 2000;31(4):662-9. [PMID: 10753273] Wang 2008 \{published data only\} Wang E, HoM J, Loe H, Kaehler N, Helgerud J. Plantar flexion: an eMective training for peripheral arterial disease. European Journal of Applied Physiology 2008;104(4):749-56. [PMID: 18726111]

Zwierska 2005 \{published data only\} Zwierska I, Walker RD, Choksy SA, Male JS, Pockley AG, Saxton JM. Upper- vs lowerlimb aerobic exercise rehabilitation in patients with symptomatic peripheral arterial disease: a randomized controlled trial. Journal of Vascular Surgery 2005;42(6):1122-30. [PMID: 16376202]

\section{References to ongoing studies}

ACTRN12616000243415 \{published data only\} ACTRN12616000243415. Comparison of the eMects of supervised treadmill walking training and supervised walking with poles on functional capabilities in patients with intermittent claudication. anzctr.org.au/Trial/Registration/ TrialReview. aspx?id=369663 (first received 23 November 2015).

NCT03837639 \{published data only\} NCT03837639. Arm-crank exercise training on cardiovascular function of patients with peripheral artery disease. clinicaltrials.gov/ct2/show/NCT03837639 (first received 12 February 2019). 


\section{Additional references}

Aboyans 2018 Aboyans V, Ricco JB, Bartelink MEL, Bjorck M, Brodmann M, Cohnert T, et al. Editor's Choice 2017 ESC Guidelines on the diagnosis and treatment of peripheral arterial diseases, in collaboration with the European Society for Vascular Surgery (ESVS). European Journal of Vascular and Endovascular Surgery 2018;55(3):305-68. [PMID: 28851596]

Atkins 2004 Atkins D, Best D, Briss PA, Eccles $M$, Falck-Ytter $Y$, Flottorp $S$, et al. Grading quality of evidence and strength of recommendations. British Medical Journal 2004;328(7454):1490-

4. Bendermacher 2007 Bendermacher $B L$, Willigendael EM, Nicolaï SP, Kruidenier LM, Welten RJ, Hendriks E, et al. Supervised exercise therapy for intermittent claudication in a communitybased setting is as eMective as clinicbased. Journal of Vascular Surgery 2007;45(6):1192-6. [PMID: 17543684]

Cohen 1988 Cohen J. Statistical Power Analysis in the Behavioral Sciences. 2nd edition. Hillsdale (NJ): Lawrence Erlbaum Associates, Inc, 1988.

Conte 2015 Conte MS, Pomposelli FB, Clair DG, Geraghty PJ, McKinsey JF, Mills JL, et al. Society for Vascular Surgery practice guidelines for atherosclerotic occlusive disease of the lower extremities: management of asymptomatic disease and claudication. Journal of Vascular Surgery 2015;61(3 Suppl):2S-41S. [PMID: 25638515]

DerSimonian 1986 DerSimonian R, Laird N. Meta-analysis in clinical trials. Controlled Clinical Trials 1986;7(3):177-88. [PMID: 3802833]
Dumville 2004 Dumville JC, Lee AJ, Smith FB, Fowkes FG. The health-related quality of life of people with peripheral arterial disease in the community: the Edinburgh Artery Study. British Journal of General Practice 2004;54(508):826-31. [PMID: 15527608]

Fakhry 2018 Fakhry F, Fokkenrood HJ, Spronk S, Teijink JA, Rouwet EV, Hunink MGM. Endovascular revascularisation versus conservative management for intermittent claudication. Cochrane Database of Systematic Reviews 2018, Issue 3. Art. No: CD010512. [DOI: 10.1002/14651858.CD010512.pub2] Fokkenrood 2015 Fokkenrood HJ, Lauret GJ, Verhofstad N, Bendermacher BL, Scheltinga MR, Teijink JA. The eMect of supervised exercise therapy on physical activity and ambulatory activities in patients with intermittent claudication. European Journal of Vascular and Endovascular Surgery 2015;49(2):184-91. [PMID: 25496986]

Fowkes 2013 Fowkes FG, Rudan D, Rudan I, Aboyans V, Denenberg JO, McDermott $M M$, et al. Comparison of global estimates of prevalence and risk factors for peripheral artery disease in 2000 and 2010: a systematic review and analysis. Lancet (London, England) 2013;382(9901):1329-40. [PMID: 23915883]

Gerhard-Herman 2017 Gerhard-Herman MD, Gornik HL, Barrett C, Barshes NR, Corriere MA, Drachman DE, et al. 2016 AHA/ACC Guideline on the management of patients with lower extremity peripheral artery disease: executive summary: a report of the American College of Cardiology/American Heart Association Task Force on Clinical Practice Guidelines. Circulation 2017;135(12):e686725. [PMID: 27840332] 
Golledge 2018 Golledge J, Maarij K, Moxon JV, Beard JD, Girold S, Wrang H, et al. Systematic review and meta-analysis of clinical trials examining the benefit of exercise programmes using Nordic walking in patients with peripheral artery disease. European Journal of Vascular Endovascular Surgery 2018;56(4):534-43.

Gommans 2015 Gommans LN, Fokkenrood HJ, Van Dalen HC, Scheltinga MR, Teijink JA, Peters RJ. Safety of supervised exercise therapy in patients with intermittent claudication. Journal of Vascular Surgery 2015;61(2):512-8.e2. [PMID: 25441008]

Gommans 2016 Gommans LN, Hageman D, Jansen I, De Gee R, Van Lummel RC, Verhofstad N, et al. Minimal correlation between physical exercise capacity and daily activity in patients with intermittent claudication. Journal of Vascular Surgery 2016;63(4):983-9. [PMID: 26806522]

GRADEproGDT 2015 [Computer program] McMaster University (developed by Evidence Prime) GRADEproGDT. Version accessed 15 November 2019. Hamilton (ON): McMaster University (developed by Evidence Prime), 2015. Available from www.gradepro.org.

Haas 2012 Haas TL, Lloyd PG, Yang HT, Terjung RL. Exercise training and peripheral arterial disease. Comprehensive Physiology 2012;2(4):2933-3017. [PMID: 23720270]

Hageman 2018 Hageman D, Fokkenrood HJ, Gommans LN, Van den Houten MM, Teijink JA. Supervised exercise therapy versus home-based exercise therapy versus walking advice for intermittent claudication. Cochrane Database of Systematic Reviews 2018, Issue 4. Art. No: CD005263. [DOI: 10.1002/14651858. CD005263.pub4]
Harwood 2016 Harwood AE, Cayton T, Sarvanandan R, Lane R, Chetter I. A review of the potential local mechanisms by which exercise improves functional outcomes in intermittent claudication. Annals of Vascular Surgery 2016;30:31220. [PMID: 26362620]

Higgins 2019 Higgins JP, Thomas J, Chandler J, Cumpston M, Li T, Page MJ, Welch VA (editors). Cochrane Handbook for Systematic Reviews of Interventions Version 6.0 (updated July 2019). Cochrane, 2019. Available from www.training. cochrane.org/handbook.

Jansen 2019 Jansen SCP, Hoorweg BBN, Hoeks $\mathrm{SE}$, Van den Houten MML, Scheltinga MRM, Teijink JAW, et al. A systematic review and meta-analysis of the effects of supervised exercise therapy on modifiable cardiovascular risk factors in intermittent claudication. Journal of Vascular Surgery 2019;69(4):1293-1308. e2. [PMID: 30777692]

Kruidenier 2009 Kruidenier LM, Nicolai $\mathrm{SP}$, Hendriks EJ, Bollen EC, Prins $\mathrm{MH}$, Teijink JA. Supervised exercise therapy for intermittent claudication in daily practice. Journal of Vascular Surgery 2009;49(2):363-70. [PMID: 19028059]

Lane 2017 Lane R, Harwood A, Watson $L$, Leng GC. Exercise for intermittent claudication. Cochrane Database of Systematic Reviews 2017, Issue 12. Art. No: CD000990. [DOI: 10.1002/14651858. CD000990.pub4]

Lefebvre 2011 Lefebvre C, Manheimer E, Glanville J. Chapter 6: Searching for studies. In: Higgins JP, Green S editor(s). Cochrane Handbook for Systematic Reviews of Interventions Version 5.1.0 (updated March 2011). The Cochrane Collaboration, 2011. Available from handbook.cochrane.org. 
McDermott 2001 McDermott MM, Greenland P, Liu K, Guralnik JM, Criqui MH, Dolan NC, et al. Leg symptoms in peripheral arterial disease: associated clinical characteristics and functional impairment. Journal of the American Medical Association 2001;286(13):1599-606. [PMID: 11585483]

Nicolaï 2010 Nicolaï SP, Teijink JA, Prins MH, Exercise Therapy in Peripheral Arterial Disease Study Group. Multicenter randomized clinical trial of supervised exercise therapy with or without feedback versus walking advice for intermittent claudication. Journal of Vascular Surgery 2010;52(2):348-55. [PMID: 20478681]

Parmenter 2011 Parmenter BJ, Raymond J, Dinnen P, Singh MA. A systematic review of randomized controlled trials: walking versus alternative exercise prescription as treatment for intermittent claudication. Atherosclerosis 2011;218(1):1-12. [PMID: 21601857]

Parmenter 2013 Parmenter BJ, Raymond J, Fiatarone Singh MA. The eMect of exercise on fitness and performancebased tests of function in intermittent claudication: a systematic review. Sports Medicine (Auckland, NZ) 2013;43(6):51324. [PMID: 23539309]

Parmenter 2015 Parmenter BJ. Exercise training for management of peripheral arterial disease: a systematic review and meta-analysis. Sports Medicine 2015;45(2):231-44.

Piercy 2018 Piercy KL, Troiano RP, Ballard RM, Carlson SA, Fulton JE, Galuska DA, et al. The Physical Activity Guidelines for Americans. JAMA 2018;320(19):2020-8. [PMID: 30418471]

Review Manager 2020 [Computer program] The Cochrane Collaboration Review Manager 5 (RevMan 5). Version 5.4. Copenhagen: The Cochrane Collaboration, 2020.
Tompra 2015 Tompra N, Foster C, SanchisGomar F, De Koning JJ, Lucia A, Emanuele E. Upper versus lower limb exercise training in patients with intermittent claudication: a systematic review. Atherosclerosis 2015;239(2):599-606.

Treat-Jacobson 2019 Treat-Jacobson D, McDermott MM, Bronas UG, Campia $\mathrm{U}$, Collins TC, Criqui MH, et al. Optimal exercise programs for patients with peripheral artery disease: a scientific statement from the American Heart Association. Circulation 2019;139(4):e1033. [PMID: 30586765]

\section{References to other published ver- sions of this review}

Lauret 2012 Lauret GJ, Fakhry F, Fokkenrood HJP, Hunink MG, Teijink JAW, Spronk S. Modes of exercise training for intermittent claudication. Cochrane Database of Systematic Reviews 2012, Issue 2. Art. No: CD009638. [DOI: 10.1002/14651858.CD009638]

Lauret 2014 Lauret GJ, Fakhry F, Fokkenrood HJP, Hunink MGM, Teijink JAW, Spronk $S$. Modes of exercise training for intermittent claudication. Cochrane Database of Systematic Reviews 2014, Issue 7. Art. No: CD009638. [DOI: 10.1002/14651858.CD009638.pub2] 


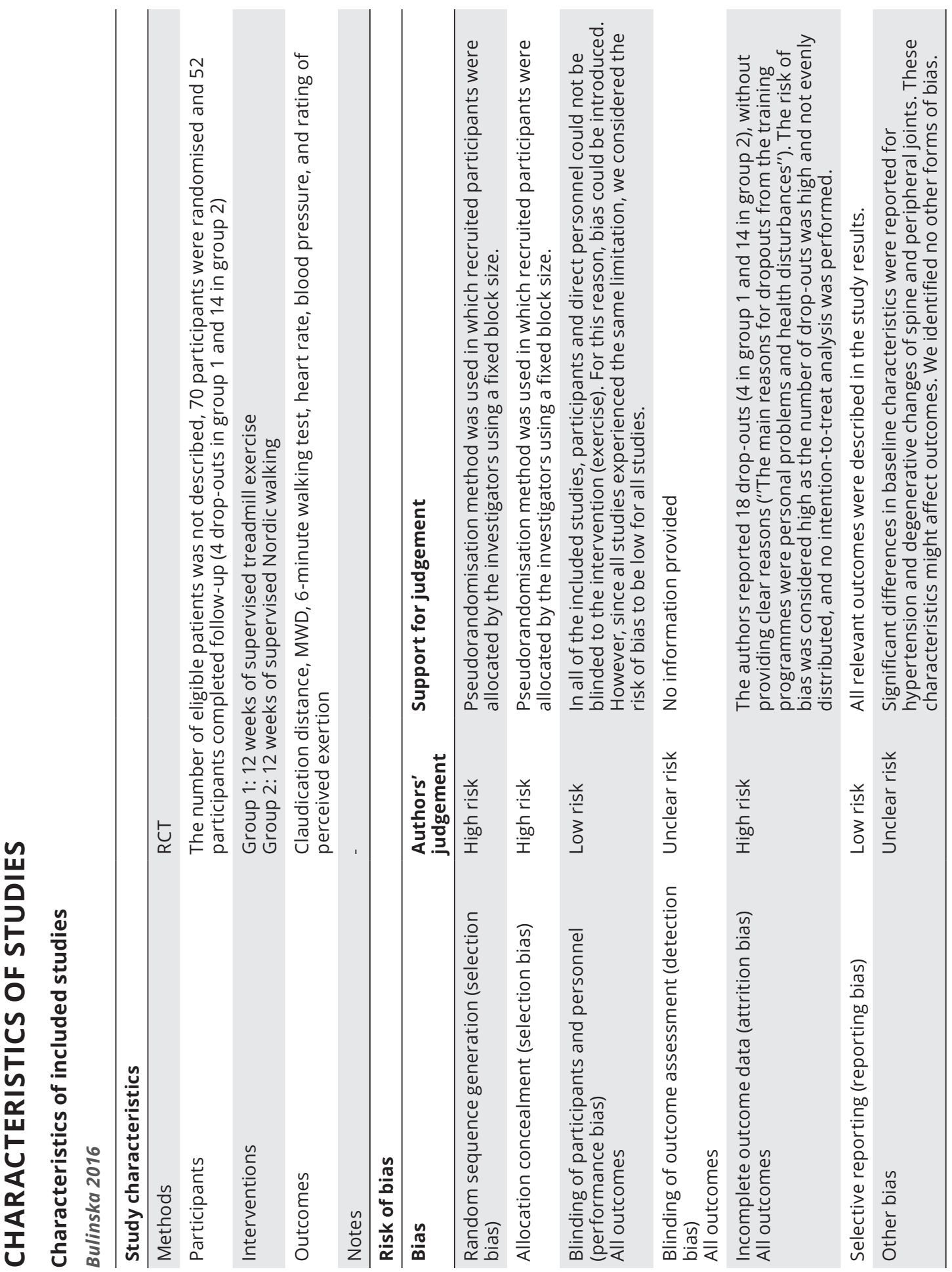




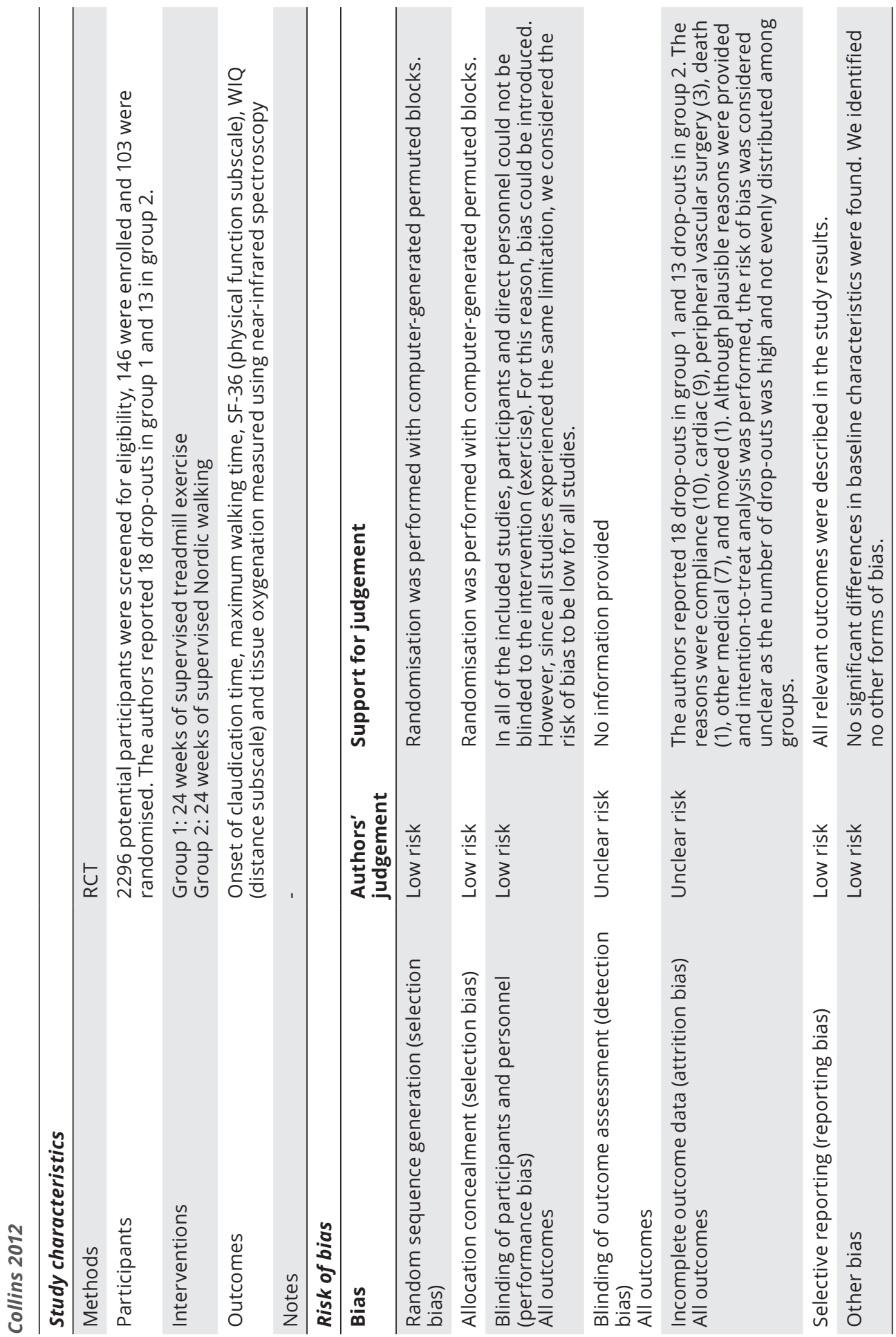




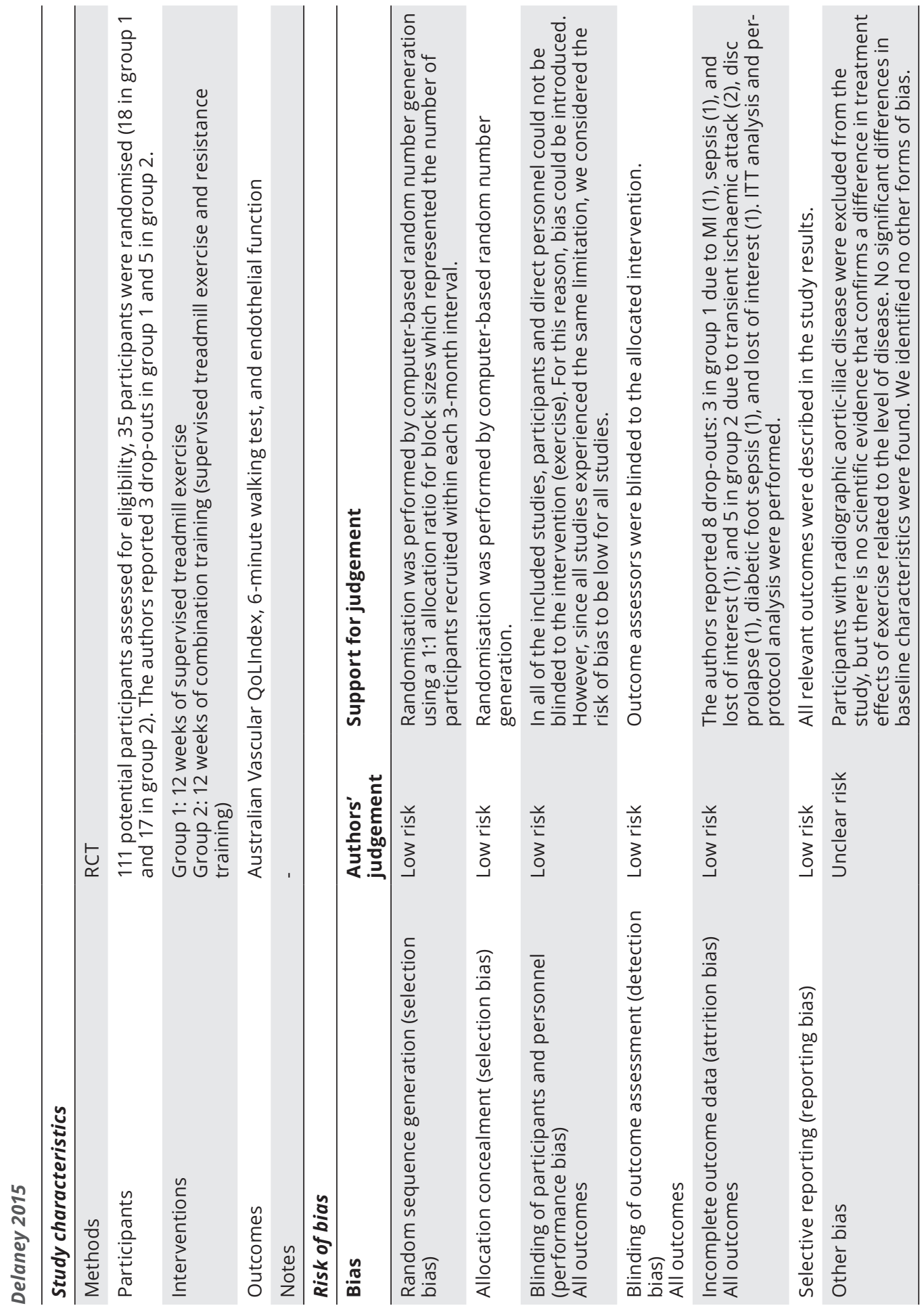




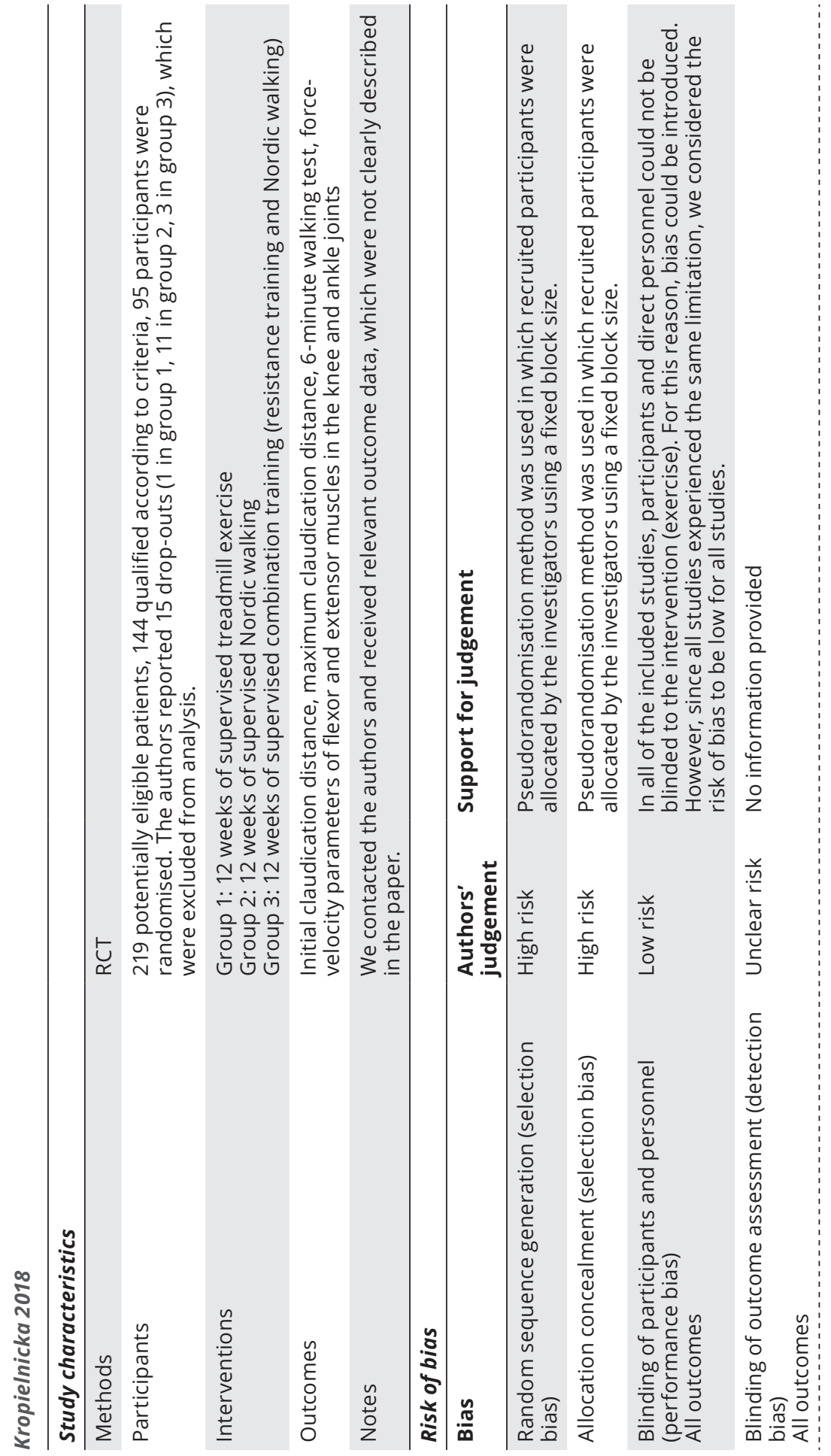




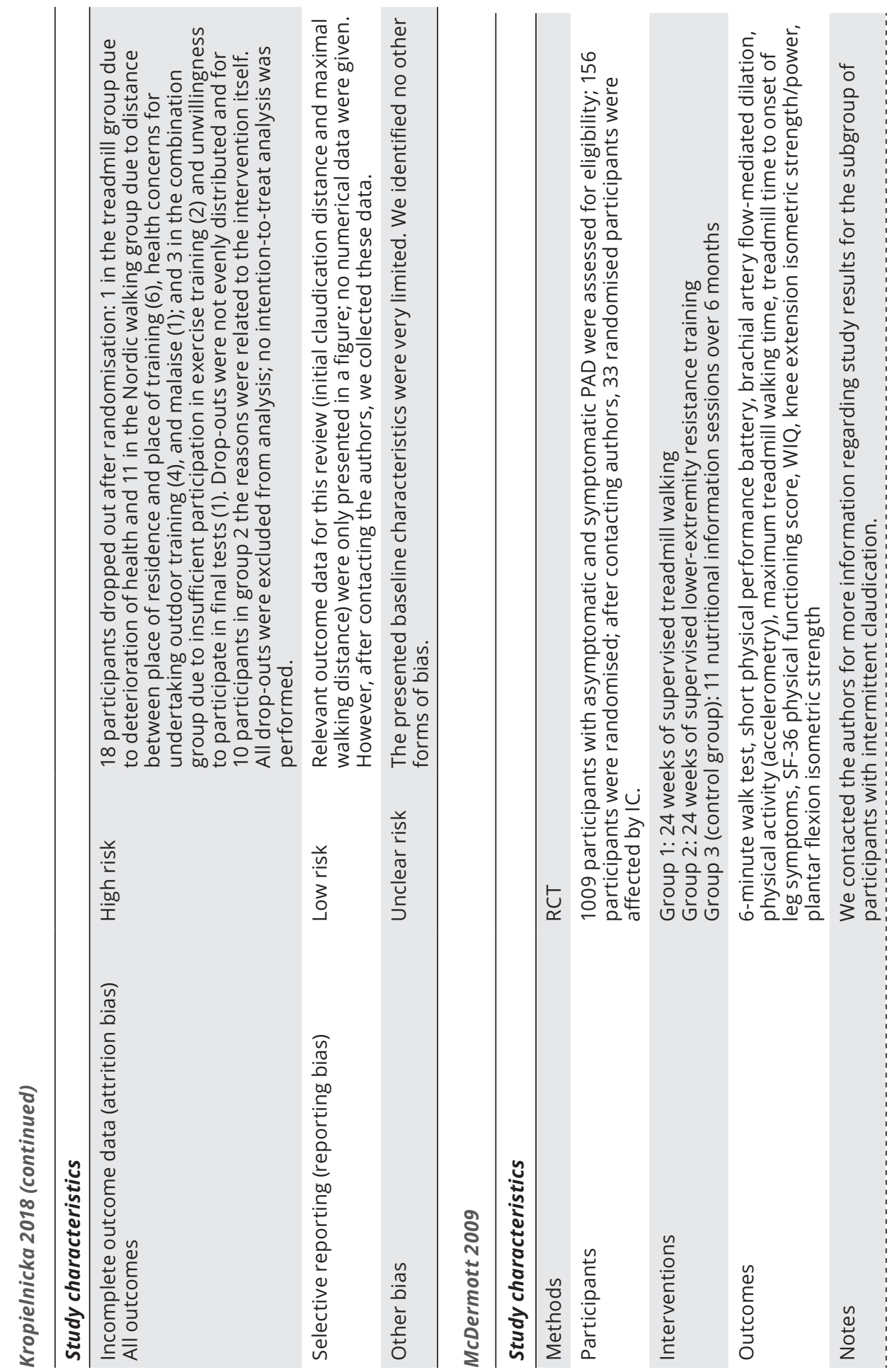




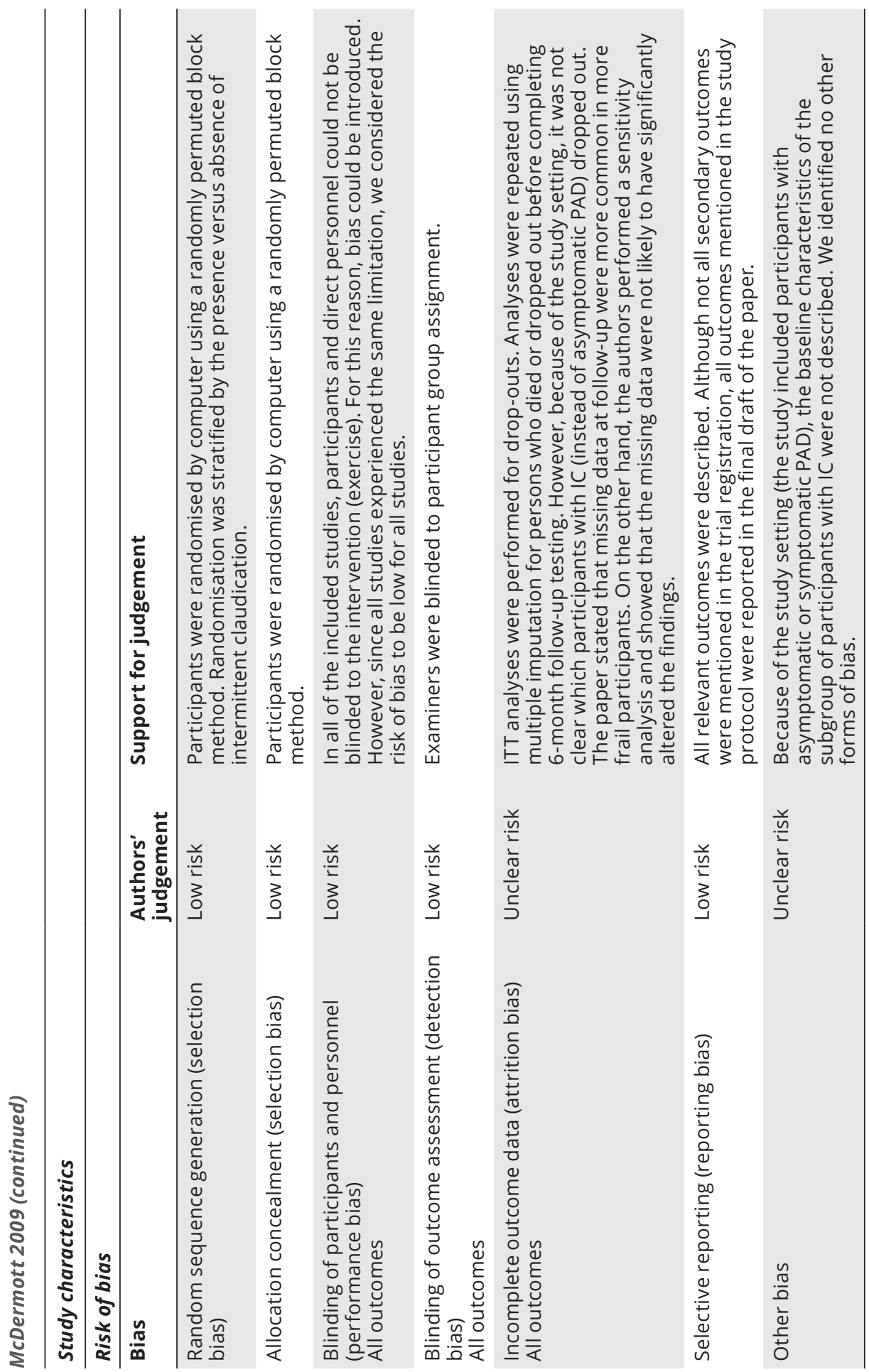



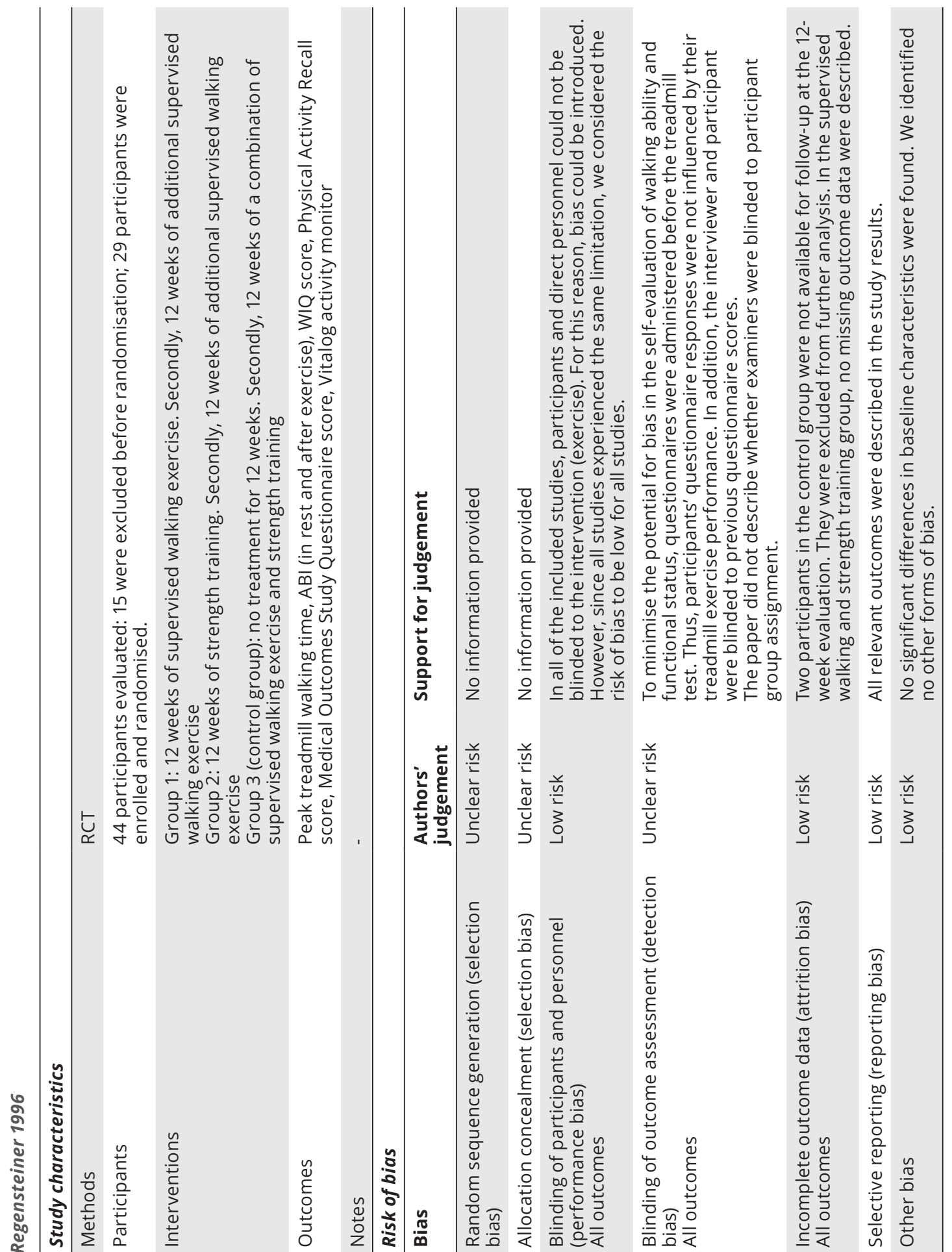


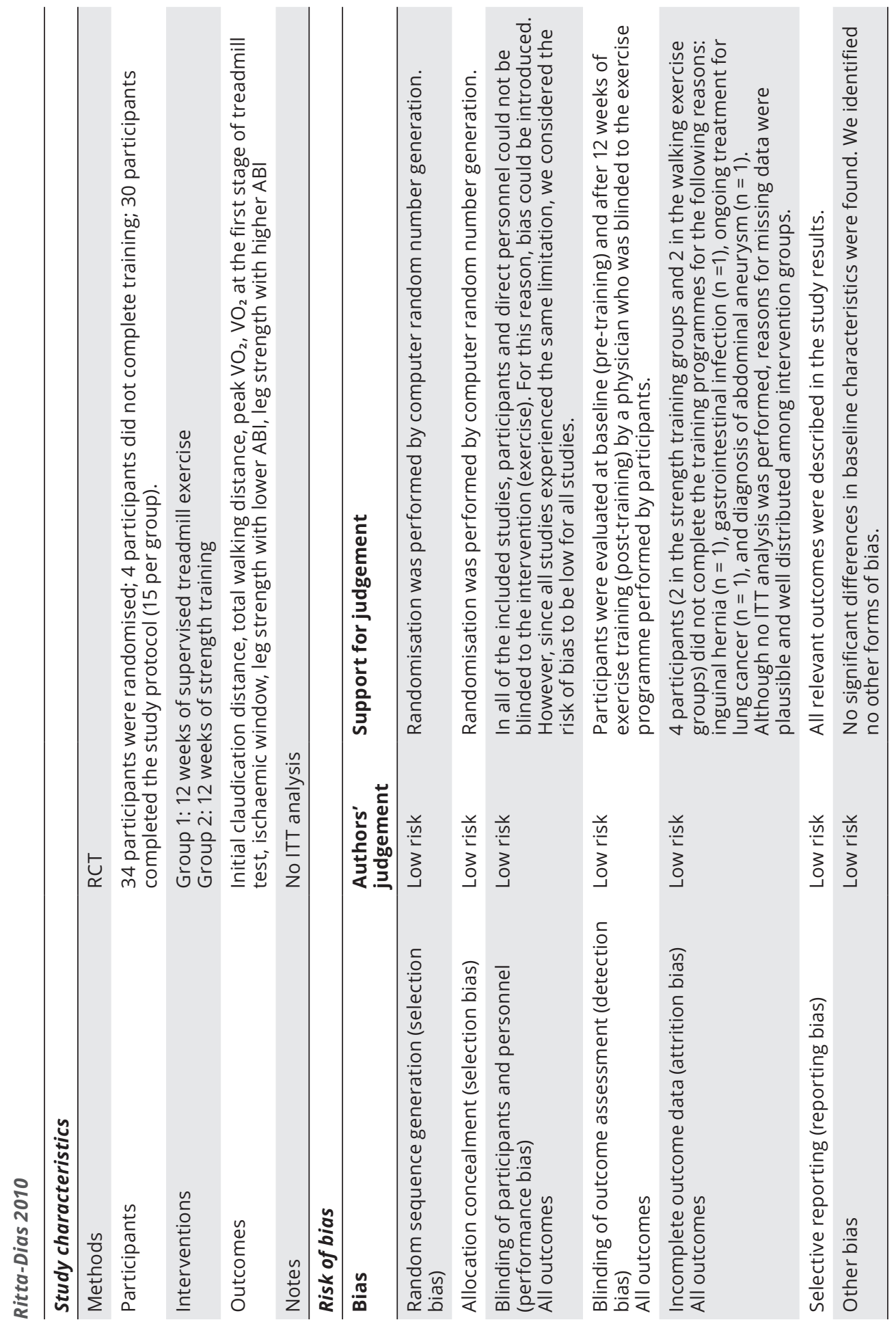




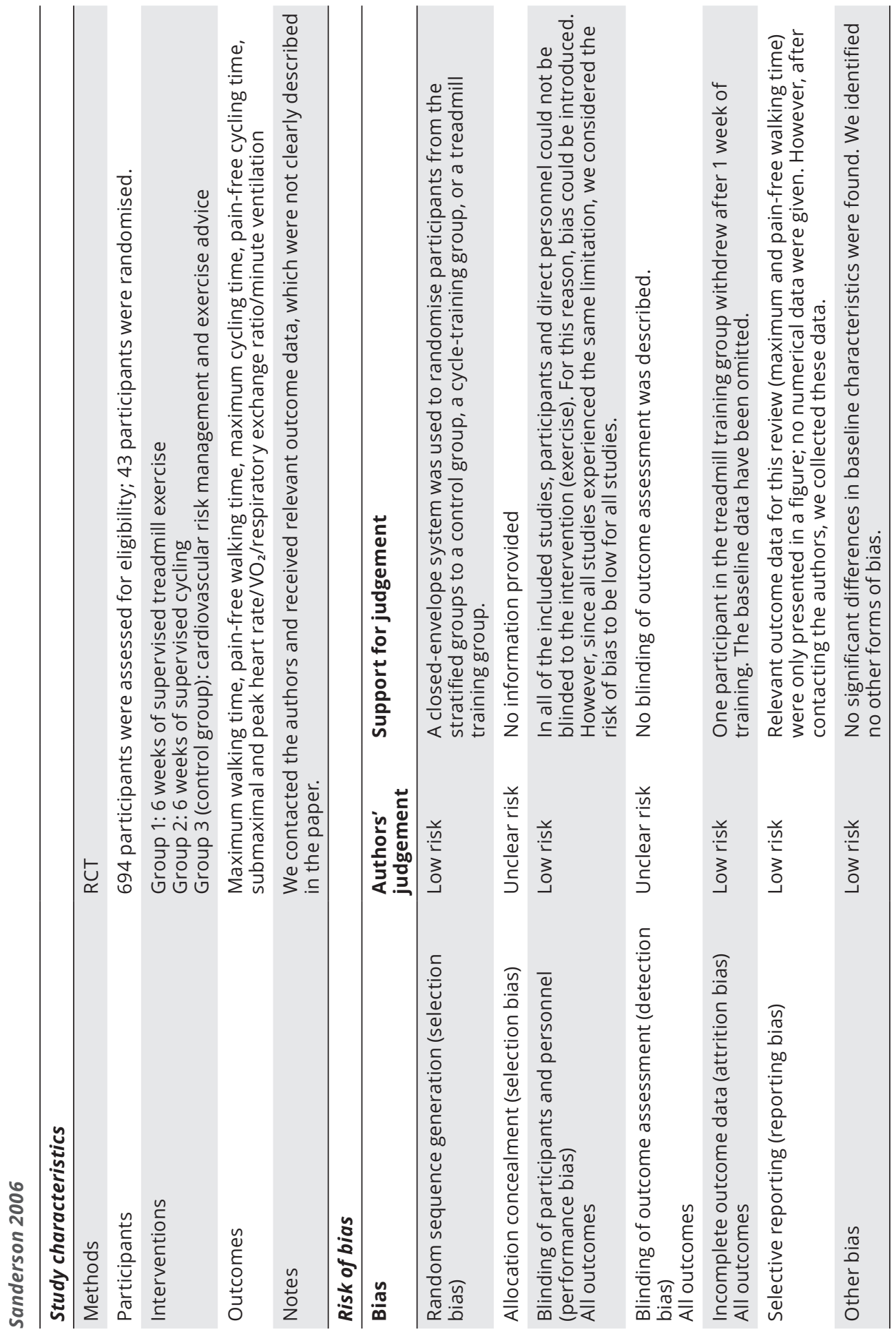




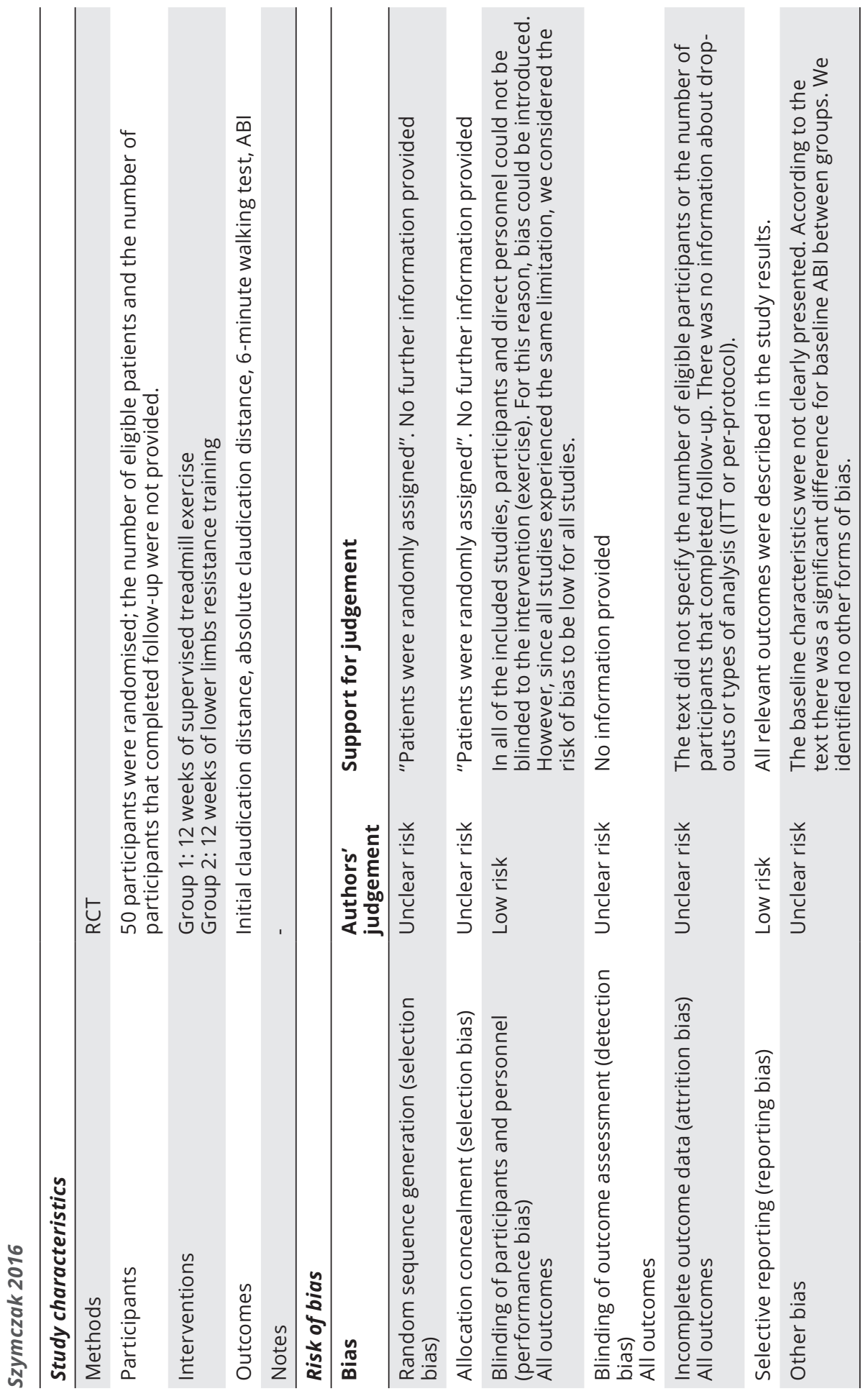



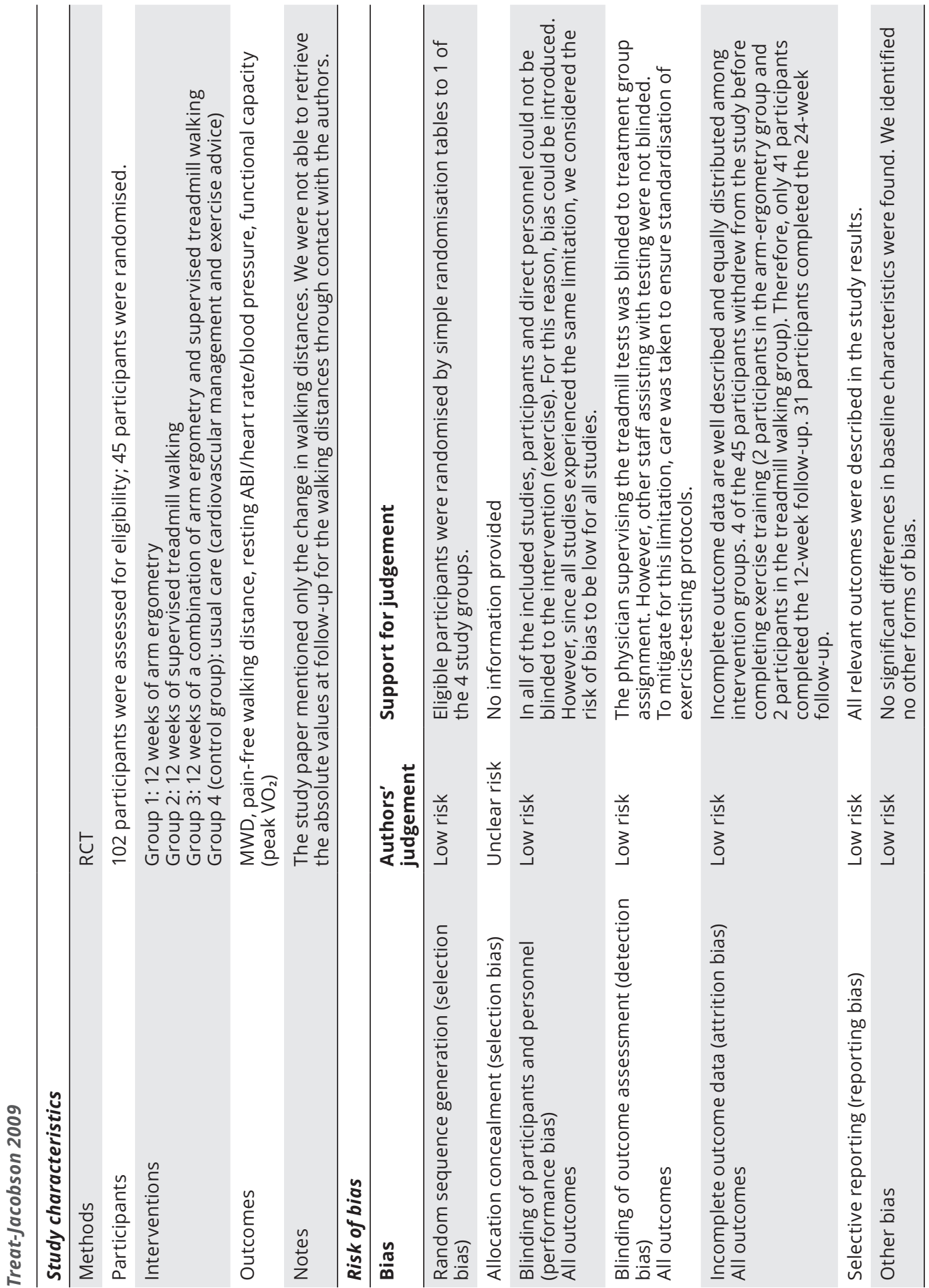
Characteristics of excluded studies

\begin{tabular}{|c|c|}
\hline Study & Reason for exclusion \\
\hline Dedes 2010 & Meeting poster; no journal article available \\
\hline Delaney 2014 & No correct outcome measures \\
\hline EXERT Study 2018 & $\begin{array}{l}\text { The original results of the EXERT study are not published. } \\
\text { Only one publication is available comparing long-term } \\
\text { follow-up ( } 1 \text { to } 4 \text { years) to end of study results ( } 24 \text { weeks). } \\
\text { Baseline data were not available from the authors. }\end{array}$ \\
\hline Gardner 2011 & Commentary on other journal article; not an RCT \\
\hline Jones 1996 & $\begin{array}{l}\text { Outcome measures unclearly described; no information } \\
\text { from authors }\end{array}$ \\
\hline Kao 2012 & Meeting poster; no article available (authors contacted) \\
\hline Kim 2006 & Commentary on other journal article; not an RCT \\
\hline Kuwabara 2010 & Meeting poster; no article available \\
\hline Nawaz 2001 & $\begin{array}{l}\text { No intervention group with adequate supervised walking } \\
\text { therapy; no correct outcome measures }\end{array}$ \\
\hline Oakley 2008 & $\begin{array}{l}\text { No intervention group with adequate supervised walking } \\
\text { therapy }\end{array}$ \\
\hline Ornelas 2011 & Meeting poster; no article available \\
\hline Parmenter 2013 & No control group with SET \\
\hline Parr 2009 & $\begin{array}{l}\text { No intervention group with adequate supervised walking } \\
\text { therapy }\end{array}$ \\
\hline Roitman 2010 & Editorial; not an RCT \\
\hline Saxton 2008 & $\begin{array}{l}\text { No control group with adequate SET; no correct outcome } \\
\text { measures }\end{array}$ \\
\hline Saxton 2011 & $\begin{array}{l}\text { No control group with adequate SET; no correct outcome } \\
\text { measures (MWD assessed by shuttle-walk test instead } \\
\text { of protocolised treadmill test) }\end{array}$ \\
\hline Tebbutt 2011 & No control group with adequate SET \\
\hline Treat-Jacobson 2011 & $\begin{array}{l}\text { No relevant outcome measures for this review assessed and } \\
\text { reported }\end{array}$ \\
\hline Treat-Jacobson 2012 & $\begin{array}{l}\text { Meeting poster; no journal article available; no control group } \\
\text { with adequate SET }\end{array}$ \\
\hline Van Schaardenburgh 2017 & No control group with adequate SET \\
\hline Walker 2000 & $\begin{array}{l}\text { No control group with adequate SET; no correct outcome } \\
\text { measures (no treadmill test) }\end{array}$ \\
\hline Wang 2008 & No control group with SET \\
\hline Zwierska 2005 & No control group with adequate SET \\
\hline
\end{tabular}




\section{DIFFERENCES BETWEEN PROTOCOL AND REVIEW}

For this update, in the Types of interventions section, 'Nordic walking' was added as an alternative mode of exercise. In the original protocol and review, studies comparing different types of walking exercise were not included, and Nordic walking was considered a different type of walking exercise. Recent publications have led to a different insight, as Nordic walking is different from standard walking because it uses a core-focused walking technique which engages the muscles of the arms and trunk to reduce the load on the legs during walking. Therefore, the criterion for not including studies was changed to 'different settings of walking exercise' (supervised versus unsupervised, community-based versus hospital), and Nordic walking was included as an alternative mode of exercise. In the Data collection and analysis section, a different method to analyse treatment effect was used in this update. In the case of different treadmill test protocols, the standardised mean difference approach was used for summary statistics. Therefore, it was no longer necessary to convert times or distances to metabolic equivalent of tasks (METs).

In the previous version, we altered the primary outcome from mean change in maximum or pain-free walking distance to a post-intervention maximum or pain-free walking distance. After extracting the data, most of the included trials only reported pre-intervention and post-intervention walking distances and their variances. No data on mean change were reported. Although calculating the mean change in walking distance from the pre-intervention and post-intervention data was possible, calculating a variance (standard deviation) of the mean change without having individual participant data was more challenging. To calculate a variance of the mean change from the reported summary pre-intervention and post-intervention variances, we needed at least the correlation between the preintervention and post-intervention variance (Higgins 2019), which was not reported. Furthermore, as we only included randomised trials and no significant difference in walking distance between the intervention and control group in each trial existed at baseline, we did not have any indication that our main outcome, the mean difference in post-intervention walking distance between the intervention and control group might be biased. We changed the $P$ value that was considered statistically significant in case of heterogeneity from 0.05 in our protocol to 0.10 in our full review. In the Background, we adjusted the description of the systematic review by Parmenter and colleagues (Parmenter 2011) after they contacted us and added why no metaanalysis was performed in this systematic review. 



\section{Summary and discussion}

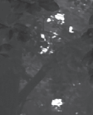

- tas.
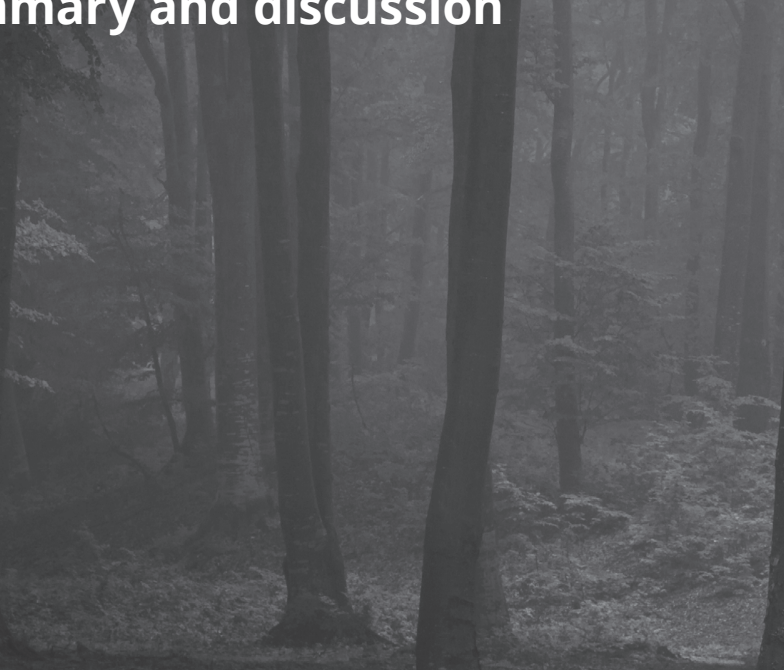

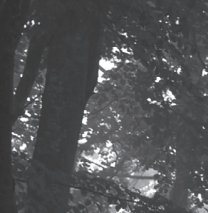
sto

if: 8

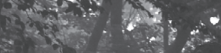

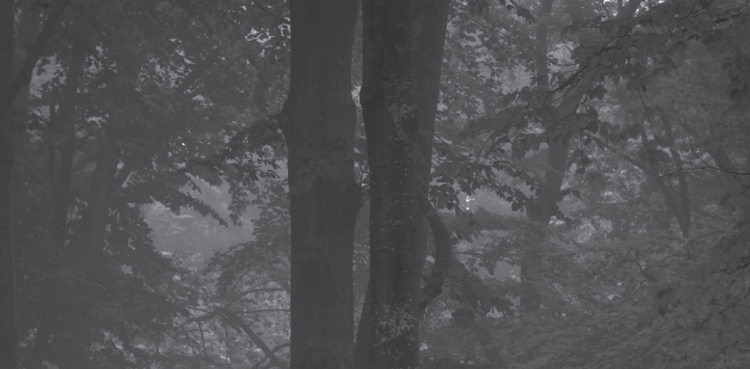





\section{Chapter}

Summary, general discussion, conclusions, recommendations and future perspectives 
In this thesis, several studies are presented that may aid in optimising supervised exercise therapy (SET) with emphasis on personalisation of treatment and cardiovascular risk management in patients with intermittent claudication (IC). The first part covers introductory remarks that set the stage for the studies that are presented in the second and third parts of the thesis. The second part contains two chapters that are dedicated to the effects of SET on cardiovascular risk, secondary interventions and mortality. Finally, the third part contains four studies that primarily cover the suitability of SET in IC patients.

\section{PART I Introduction}

Peripheral artery disease (PAD) is caused by systemic atherosclerosis affecting the infrarenal aorta and lower limb arteries and usually occurs after the age of 50. The classical presentation of PAD is intermittent claudication (IC) defined as leg pain during walking that is relieved upon a short period of rest. The 5-year mortality risk is $10-15 \%$, mostly due to fatal cardiovascular events, whereas an additional $30 \%$ of patients are confronted with a non-fatal cardiovascular event. ${ }^{1-4}$ Therefore, the main goals of IC treatment are symptom relief and cardiovascular risk management (CVRM). Current guidelines recommend SET as initial treatment for IC and support a stepped care approach in which revascularization is reserved when SET is not successful. ${ }^{1,5}$ The general aim of this thesis was to explore if SET could be optimised as a lifestyle intervention with emphasis on personalisation of treatment and CVRM.

\section{PART II Studies on the potential influence of supervised exercise therapy on cardiovascular risk, secondary in- terventions and mortality}

PAD is associated with an increased risk of cardiovascular and all-cause mortality, even after adjustment for other cardiovascular risk factors. ${ }^{6,7}$ This finding underscores the importance of CVRM in IC treatment. Next to medication and lifestyle advice, exercise has been demonstrated to exert positive effects on cardiovascular disease and associated risk factors. ${ }^{8-11}$ As a consequence, exercise training is recommended as a central component of rehabilitation and preventive interventions. ${ }^{1,5,11-13}$ Chapter 2 reports on a systematic review and meta-analysis of prospective studies including 27 studies ( $n=808$ patients) that evaluates the effects of SET on cardiovascular risk factors in IC patients. This . Pooled mean differences between follow-up and baseline were analysed using a random-effects model for the following risk factors: blood pressure (systolic or diastolic), heart rate, lipid profile (total cholesterol, triglycerides, high-density lipoprotein cholesterol, lowdensity lipoprotein cholesterol), glucose, glycated haemoglobin, body weight, body mass index, and cigarette smoking. After 6 weeks to 3 months of SET, systolic blood pressure decreased with $4 \mathrm{~mm} \mathrm{Hg}$ and diastolic blood pressure with $2 \mathrm{~mm} \mathrm{Hg}$. After 
6 to 12 months of SET, low-density lipoprotein cholesterol and total cholesterol both decreased by $0.2 \mathrm{mmol} / \mathrm{L}$. These findings support the prescription of SET programs not only to increase walking distances but also for their associated beneficial effects on a number of cardiovascular risk factors.

Although SET is recommended as primary treatment, guideline adherence is not yet self-evident in daily practice. ${ }^{14-16}$ In the Netherlands, availability and accessibility of SET are optimized through a nationwide community-based organization. Furthermore, SET is fully reimbursed and increased awareness of referring physicians probably improved SET referral rates. ${ }^{17,18}$ These factors have most likely contributed to the considerable increase of SET as primary treatment (from 63\% in 2013 to $87 \%$ in 2017), as shown in a nationwide retrospective data analysis of health insurance claims of 54504 newly diagnosed IC patients (Chapter 3). Multiple regression analysis demonstrated that patients who underwent endovascular revascularisation (ER) or open surgery as a primary treatment had a higher risk of secondary revascularizations (hazard ratio (HR) 1.44 and HR 1.45, respectively) and demonstrated a higher mortality risk compared to SET as a primary treatment (HR 1.38 and HR 1.49, respectively). Although this analysis had its restrictions, it provides a unique insight into real-world data and illustrates the potential beneficial long-term effects of the 'exercise-first strategy'.

\section{PART III Suitability of supervised exercise therapy in patients with intermittent claudication}

SET is generally regarded as an effective treatment for IC, but the question arises whether it is the most suitable therapy for each IC patient. Although three randomized controlled trials comparing SET to ER for aortoiliac stenoses found similar results regarding walking performance and quality of life $\mathrm{e}^{19-21}$, location of stenosis is a commonly used argument for a revascularisation-first strategy. The ELECT Registry aimed to provide insight into the discussion questioning the effectiveness of SET for aortoiliac disease. Chapter 4 covers the rationale and protocol for this multicentre prospective observational study. Chapter $\mathbf{5}$ depicts the results of this unique effort. A total of 267 patients were studied, of which $26 \%$ suffered from aortoiliac disease and $43 \%$ of femoropopliteal disease. Patients were primarily treated with SET according to current guidelines. All patients demonstrated equal improvements in walking performance and health related quality of life after 3 and 6 months of SET, regardless of stenosis/occlusion location. This study confirms that, ideally, all IC patients should receive a trial of SET before possibly considering invasive treatment, regardless of location or extent of the stenosis/occlusion.

Other potentially moderating influences on the effectiveness of SET are psychological constructs. In other words, does the psychological make-up of an IC patient determine outcomes of SET? A substudy of the ELECT Registry that is presented in Chapter 6 analysed possible associations between SET-related 
outcomes (walking distances, Six-Minute Walk Test, and VascuQol-6) and a set of psychological factors (extraversion, neuroticism, conscientiousness, anxiety, depression, self-control, optimism, and self-efficacy). Multiple linear regression analyses demonstrated a limited number of small overall effects of questionable clinical relevance. Interestingly however, these investigated factors did not affect (in either direction) the clinical course after 6 months of follow-up. Furthermore, self-efficacy did not improve during 12 months of follow-up. Consequently, it is concluded that the beneficial effects of SET occur regardless of the psychological constructs of an IC patient.

In addition to studying the spectrum of indications of SET, the mode of exercise may also be relevant. SET often consists of treadmill or track walking. However, not all patients with IC are able to complete such exercise protocols because of concomitant comorbidity (e.g. osteoarthritis, pulmonary disease, cardiac disease). Alternative modes of supervised exercise training do exist and are also associated with improved walking capacity. A Cochrane review in Chapter 7 included 10 studies with a total of 527 randomised IC participants. The investigated alternative modes of exercise were cycling, lower-extremity resistance training, upper-arm ergometry, Nordic walking, and combinations of exercise modes. No clear differences were detected between alternative exercise modes and supervised walking exercise with respect to improving maximum and pain-free walking distance. These findings indicate that alternative exercise modes may also be useful when walking is not a suitable option. Because the certainty of evidence was judged to be low, mainly due to small sample size and risk of bias concerns, it was not possible to conclude that the alternative modes are equally effective to walking for all IC patients.

\section{CONCLUSIONS OF THIS THESIS}

1. Supervised exercise therapy (SET) has favourable effects on modifiable cardiovascular risk factors, specifically blood pressure and cholesterol levels.

2. The exercise-first approach is successfully implemented in the Netherlands, as $87 \%$ of patients with intermittent claudication (IC) received a primary course of SET in 2017.

3. Patients receiving primary SET had fewer lower limb revascularisations and demonstrated better survival than patients undergoing primary endovascular revascularisation or open surgery.

4. Location of stenosis/occlusion does not affect short-term effectiveness of SET.

5. The beneficial effects of SET occur regardless of certain psychological constructs, including depression and anxiety.

6. Alternative exercise modes may be useful when supervised walking exercise is not a suitable option, e.g. due to common comorbidities. 
In conclusion, the overall findings of this thesis indicate that there are no clinical patient characteristics that consistently identify individuals with IC who will not benefit from walking exercise. Therefore it supports current guidelines recommending a SET-first strategy in every IC patient.

\section{RECOMMENDATIONS AND FUTURE PERSPECTIVES}

\section{Exercise-first approach}

The research of this thesis was conducted in the Netherlands, a country with sufficient SET availability. It proves that $>80 \%$ adherence to the exercisefirst strategy is feasible. Given the effectiveness of SET, including risk-benefit considerations, efforts should be made to make exercise accessible to all patients with IC worldwide. ${ }^{1,5,22-24}$ As is rightly pointed out in an invited commentary, longterm data for functional outcomes and quality of life in this "highly developed Dutch SET system" are desirable. ${ }^{25}$ Furthermore, information on the willingness to participate and adherence to SET programs is of interest. Hopefully, the long-term data of the ELECT Registry will fill some of these residual evidence gaps.

Additionally, a new focus for improvement was identified in chapter 3. Primary revascularisation should be accompanied by SET as efforts to enhance physical capacity should always be included in IC treatment., In this thesis, a disappointingly low $10 \%$ received SET in the first year following primary revascularisation. Future endeavours should be directed towards increasing this rate of participation.

As the general belief is growing that a vascular intervention as sole treatment for IC is an inferior option compared to SET, combination therapy (endovascular treatment plus SET) is a contemporary research topic. Proponents advocate a synergetic effect of these combined treatment modalities. Three recent metaanalyses did not yield unequivocal conclusions, but results suggested that combination therapy possibly gives favourable results in the short to mid-term follow-up compared to SET alone. ${ }^{26-28}$ An economic analysis of the ERASE study demonstrates that combination therapy might be more cost-effective after a 12 months of follow-up. However, patients with femoropopliteal disease or aged 65 years and beyond might benefit more from a SET only strategy. ${ }^{29}$ It seems inappropriate to expose all IC patients to invasive treatment, while SET is already highly effective in itself, especially respecting the well-known aphorism "first, do no harm". ${ }^{24,30}$ Further research is needed to determine the optimal treatment strategy for IC in the long term, considering patient characteristics, personal preferences, costs, and potential cons of invasive treatment.

\section{Promoting cardiovascular health is essential}

This thesis contains the first systematic review on the possible associations of SET and cardiovascular risk in IC. Due to the relatively small number of eligible studies, it was not possible to analyse all the risk factors of interest. Despite CVRM being 
one of the two main aims of IC treatment, cardiovascular risk does not seem to receive appropriate attention in research settings. Notably, the 5-year mortality rate in the large contemporary cohort that is presented in chapter 3 is quite similar to the $10-15 \%$ mortality rate that is based on relatively dated literature..$^{1-3}$ It would be interesting to investigate whether improvement is possible and specifically if all-cause mortality rates really drop after SET. ${ }^{25}$ Future studies should include modifiable risk factors for short-term follow-up and cardiovascular morbidity and mortality at long-term follow-up.

It is a missed opportunity if SET is not utilized for its potential positive effects on promoting cardiovascular health. The ultimate goal of SET should extend beyond confined outcomes (such as walking distances) towards actually increasing healthy behaviour such as physical activity. ${ }^{31}$ The Physical Activity Guidelines for adults also apply to older adults and to those with chronic health conditions or disabilities, to the extent that it is feasible and safe. ${ }^{32,33}$ Although it is not likely that all IC patients are able to meet these recommendations, however, if someone becomes more active, he will still gain substantial health benefits (e.g. a significant decrease in cardiovascular risk). ${ }^{32-35}$ In other words, every little bit may help.

In order to maintain and further improve the achieved results during SET, patients should be supported to continue activities beyond the program. To this end, therapy should include behavioural change techniques. ${ }^{5}$ This approach involves identifying and discussing potential barriers for each individual patient to improve the likelihood of long-term adherence. ${ }^{36-38}$ Self-efficacy is an important mediator of behaviour change, and increased self-efficacy is associated with improved cardiovascular lifestyle. ${ }^{38-42}$ Although this thesis failed to demonstrate a substantial effect of SET on self-efficacy, SET does have a potential as a lifestyle intervention. For example, its main components include mastery experiences and social modelling, both known to be able to increase self-efficacy. ${ }^{38}$ Moreover, frequent and intensive contacts between physical therapist and patient provide excellent opportunities for counselling. Nonetheless, behavioural change is a complex process that includes several motivational factors (intention, attitude, self-efficacy) which also have strong interactions. In addition, action plans and coping plans play an essential role in translating intention into actual behaviour change. ${ }^{43-45}$ Behaviour change is very challenging, and although several interventions are efficacious, even the most effective ones still have high levels of non-response. ${ }^{46}$ More attention should be paid to addressing patient-specific and situation-specific motivators and challenges to maintaining a routine walking program and physical activity. ${ }^{38,41,47-49}$

\section{Personalized treatment is the answer?}

To better align with the aim of promoting healthy behaviour, it is probably useful to identify the optimal form of exercise for individual patients. Being able to offer several modes of exercise may better suit personal preferences and could therefore improve therapy adherence. Additionally, combinations of aerobic and 
muscle strengthening activities would provide the most extensive health benefits according to the Physical Activity Guidelines. ${ }^{32,33}$ The findings in this thesis (chapter 7) indicate that alternative exercise modes may be useful when walking is not the most suitable option. More evidence is needed to substantiate the incorporation of alternative modes in SET programs. This approach also requires critical evaluation of measuring methods. Determining walking capacity solely with treadmill tests might result in an underestimation of the effect of alternative exercise modes, as treadmill walking tests have a training effect (a so-called training to the outcome measure phenomenon). ${ }^{22,50,51}$ Treadmill walking probably does not represent 'daily life' walking, for example with regard to walking surfaces and breaks. Moreover, if patients have trouble walking due to comorbidity, different measuring modes seem more suitable. ${ }^{52}$ Future studies should use validated outcome measures that allow meaningful comparisons between different modes of exercise. Conducting research into personal abilities and preferences requires applied practical studies that correlate with daily practice and consider patients on an individual level.

The findings in this thesis support a recent statement indicating that there are no clinical patient characteristics that consistently identify individuals with IC who will not benefit from walking exercise. ${ }^{22}$ Nevertheless, this does not detract from considering individual needs and preferences. For example, the high rates of anxiety and depression (percentages up to 48\%) in PAD patients require more attention, whereas a higher risk of developing depression was found during follow-up. ${ }^{53-58} \mathrm{It}$ only seems reasonable to incorporate psychological screening as part of standard procedures in order to identify patients who need further psychological evaluation and assistance. Additionally, treatment goals should better reflect patient motives, such as being self-reliant, managing chronic disease, enhancing daily life activities, and opportunities for social engagement. ${ }^{59}$ 


\section{REFERENCES}

1. Aboyans V, Ricco JB, Bartelink MEL, et al. ESC Guidelines on the Diagnosis and Treatment of Peripheral Arterial Diseases, in collaboration with the European Society for Vascular Surgery (ESVS). Eur J Vasc Endovasc Surg. 2017; 10.1016/j.ejvs.2017.07.018.

2. Criqui $\mathrm{MH}$, Langer $\mathrm{RD}$, Fronek $\mathrm{A}$, et al. Mortality over a period of 10 years in patients with peripheral arterial disease. N Engl J Med. 1992;326(6):381-386.

3. Weitz Jl, Byrne J, Clagett GP, et al. Diagnosis and treatment of chronic arterial insufficiency of the lower extremities: a critical review. Circulation. 1996;94(11):3026-3049.

4. Leng GC, Lee AJ, Fowkes FG, et al. Incidence, natural history and cardiovascular events in symptomatic and asymptomatic peripheral arterial disease in the general population. Int $J$ Epidemiol. 1996;25(6):1172-1181.

5. Gerhard-Herman MD, Gornik HL, Barrett C, et al. 2016 AHA/ACC Guideline on the Management of Patients With Lower Extremity Peripheral Artery Disease: Executive Summary: A Report of the American College of Cardiology/American Heart Association Task Force on Clinical Practice Guidelines. Circulation. 2017;135(12):e686-e725.

6. Golomb BA, Dang TT, Criqui MH. Peripheral arterial disease: morbidity and mortality implications. Circulation. 2006;114(7):688-699.

7. Criqui $\mathrm{MH}$, Aboyans V. Epidemiology of peripheral artery disease. Circ Res. 2015;116(9):1509-1526.

8. Pedersen BK, Saltin B. Exercise as medicine - evidence for prescribing exercise as therapy in 26 different chronic diseases. Scand J Med Sci Sports. 2015;25 Suppl 3:1-72.
9. Kujala UM. Evidence on the effects of exercise therapy in the treatment of chronic disease. $\mathrm{Br} J$ Sports Med. 2009;43(8):550-555.

10. European Association of Cardiovascular $P$, Rehabilitation Committee for Science G, Eacpr, et al. Secondary prevention through cardiac rehabilitation: physical activity counselling and exercise training: key components of the position paper from the Cardiac Rehabilitation Section of the European Association of Cardiovascular Prevention and Rehabilitation. Eur Heart J. 2010;31(16):1967-1974.

11. Smith SC, Jr., Benjamin EJ, Bonow RO, et al. AHA/ACCF secondary prevention and risk reduction therapy for patients with coronary and other atherosclerotic vascular disease: 2011 update: a guideline from the American Heart Association and American College of Cardiology Foundation endorsed by the World Heart Federation and the Preventive Cardiovascular Nurses Association. J Am Coll Cardiol. 2011;58(23):2432-2446.

12. Perk J, De Backer G, Gohlke H, et al. European Guidelines on cardiovascular disease prevention in clinical practice (version 2012). The Fifth Joint Task Force of the European Society of Cardiology and Other Societies on Cardiovascular Disease Prevention in Clinical Practice (constituted by representatives of nine societies and by invited experts). Eur Heart J. 2012;33(13):1635-1701.

13. Eckel RH, Jakicic JM, Ard JD, et al. 2013 AHA/ACC guideline on lifestyle management to reduce cardiovascular risk: a report of the American College of Cardiology/American Heart Association Task Force on Practice Guidelines. J Am Coll Cardiol. 2014;63(25 Pt B):2960-2984. 
14. Popplewell MA, Bradbury AW. Why do health systems not fund supervised exercise programmes for intermittent claudication? Eur J Vasc Endovasc Surg. 2014;48(6):608-610.

15. Gommans LN, Teijink JA. Attitudes to supervised exercise therapy. $\mathrm{Br} /$ Surg. 2015;102(10):1153-1155.

16. Treat-Jacobson D, McDermott MM, Beckman JA, et al. Implementation of Supervised Exercise Therapy for Patients With Symptomatic Peripheral Artery Disease: A Science Advisory From the American Heart Association. Circulation. 2019;140(13):e700-e710.

17. Hageman D, Lauret GJ, Gommans LNM, et al. Supervised Exercise Therapy for Intermittent Claudication Is Increasingly Endorsed by Dutch Vascular Surgeons. Ann Vasc Surg. 2018;47:149-156.

18. Hageman $D$, van den Houten MM, Spruijt S, Gommans LN, Scheltinga MR, Teijink JA. Supervised exercise therapy: it does work, but how to set up a program? J Cardiovasc Surg (Torino). 2017;58(2):305312.

19. Murphy TP, Cutlip DE, Regensteiner $J G$, et al. Supervised exercise versus primary stenting for claudication resulting from aortoiliac peripheral artery disease: six-month outcomes from the claudication: exercise versus endoluminal revascularization (CLEVER) study. Circulation. 2012;125(1):130-139.

20. Fakhry F, Rouwet EV, den Hoed PT, Hunink MG, Spronk S. Long-term clinical effectiveness of supervised exercise therapy versus endovascular revascularization for intermittent claudication from a randomized clinical trial. Br J Surg. 2013;100(9):1164-1171.
21. Frans FA, Bipat $S$, Reekers JA, Legemate DA, Koelemay MJ, Collaborators SS. SUPERvised exercise therapy or immediate PTA for intermittent claudication in patients with an iliac artery obstruction--a multicentre randomised controlled trial; SUPER study design and rationale. Eur J Vasc Endovasc Surg. 2012;43(4):466-471.

22. Treat-Jacobson D, McDermott MM, Bronas UG, et al. Optimal Exercise Programs for Patients With Peripheral Artery Disease: A Scientific Statement From the American Heart Association. Circulation. 2019;139(4):e10-e33.

23. Polonsky TS, McDermott MM. Lower Extremity Peripheral Artery Disease Without Chronic LimbThreatening Ischemia: A Review. JAMA. 2021;325(21):2188-2198.

24. Secemsky EA, Shen C, Schermerhorn $\mathrm{M}$, Yeh RW. Longitudinal Assessment of Safety of Femoropopliteal Endovascular Treatment With Paclitaxel-Coated Devices Among Medicare Beneficiaries: The SAFE-PAD Study. JAMA Intern Med. 2021;181(8):1071-1080.

25. Nordanstig J, Back M. Game, SET, and Match? Eur J Vasc Endovasc Surg. 2020;60(6):888.

26. Pandey A, Banerjee S, Ngo C, et al. Comparative Efficacy of Endovascular Revascularization Versus Supervised Exercise Training in Patients With Intermittent Claudication: Meta-Analysis of Randomized Controlled Trials. JACC Cardiovasc Interv. 2017;10(7):712-724.

27. Saratzis A, Paraskevopoulos I, Patel $S$, et al. Supervised Exercise Therapy and Revascularization for Intermittent Claudication: Network Meta-Analysis of Randomized Controlled Trials. JACC Cardiovasc Interv. 2019;12(12):1125-1136. 
28. Fakhry F, Fokkenrood HJ, Spronk S, Teijink JA, Rouwet EV, Hunink MGM. Endovascular revascularisation versus conservative management for intermittent claudication. Cochrane Database Syst Rev. 2018;3:CD010512.

29. Fakhry F, Rouwet EV, Spillenaar Bilgen $R$, et al. Endovascular Revascularization Plus Supervised Exercise Versus Supervised Exercise Only for Intermittent Claudication: A Cost-Effectiveness Analysis. Circ Cardiovasc Interv. 2021;14(7):e010703.

30. McDermott MM. Reducing Disability in Peripheral Artery Disease: The Role of Revascularization and Supervised Exercise Therapy. JACC Cardiovasc Interv. 2019;12(12):1137-1139.

31. Fokkenrood HJ, Lauret GJ, Verhofstad $N$, Bendermacher BL, Scheltinga MR, Teijink JA. The effect of supervised exercise therapy on physical activity and ambulatory activities in patients with intermittent claudication. Eur J Vasc Endovasc Surg. 2015;49(2):184-191.

32. Piercy KL, Troiano RP, Ballard RM, et al. The Physical Activity Guidelines for Americans. JAMA. 2018;320(19):20202028.

33. Bull FC, Al-Ansari SS, Biddle $S$, et al. World Health Organization 2020 guidelines on physical activity and sedentary behaviour. $\mathrm{Br} J$ Sports Med. 2020;54(24):1451-1462.

34. Sattelmair J, Pertman J, Ding EL, Kohl HW, 3rd, Haskell W, Lee IM. Dose response between physical activity and risk of coronary heart disease: a meta-analysis. Circulation. 2011;124(7):789-795.

35. Lear SA, Hu W, Rangarajan S, et al. The effect of physical activity on mortality and cardiovascular disease in 130000 people from 17 high-income, middle-income, and low-income countries: the PURE study. Lancet. 2017;390(10113):2643-2654.
36. Galea MN, Bray SR, Ginis KA. Barriers and facilitators for walking in individuals with intermittent claudication. J Aging Phys Act. 2008;16(1):69-83; quiz 84.

37. Barbosa JP, Farah BQ, Chehuen M, et al. Barriers to physical activity in patients with intermittent claudication. Int J Behav Med. 2015;22(1):70-76.

38. Bandura A. Health promotion by social cognitive means. Health Educ Behav. 2004;31(2):143-164.

39. Banik A, Schwarzer R, Knoll N, Czekierda K, Luszczynska A. Self-efficacy and quality of life among people with cardiovascular diseases: A meta-analysis. Rehabil Psychol. 2018;63(2):295-312.

40. Sol BG, van der Graaf $Y$, van Petersen R, Visseren FL. The effect of self-efficacy on cardiovascular lifestyle. Eur J Cardiovasc Nurs. 2011;10(3):180-186.

41. Miller, W., Rollnick, S. (2002). Motivational interviewing: Preparing people for change (2nd ed.). New York, NY: Guilford Press.

42. Sheeran P, Maki A, Montanaro E, et al. The impact of changing attitudes, norms, and self-efficacy on health-related intentions and behavior: A meta-analysis. Health Psychol. 2016;35(11):1178-1188.

43. Peels DA, Verboon $P$, van Stralen MM, et al. Motivational factors for initiating and maintaining physical activity among adults aged over fifty targeted by a tailored intervention. Psychol Health. 2020;35(10):1184-1206.

44. Schwarzer R, Lippke S, Luszczynska A. Mechanisms of health behavior change in persons with chronic illness or disability: the Health Action Process Approach (HAPA). Rehabil Psychol. 2011;56(3):161170.

45. de Vries H, Eggers SM, Bolman C. The role of action planning and plan enactment for smoking cessation. BMC Public Health. 2013;13:393. 
46. Kim ES, Kubzansky LD, Soo J, Boehm JK. Maintaining Healthy Behavior: a Prospective Study of Psychological WellBeing and Physical Activity. Ann Behav Med. 2017;51(3):337-347.

47. Rejeski WJ, Spring B, Domanchuk K, et al. A group-mediated, home-based physical activity intervention for patients with peripheral artery disease: effects on social and psychological function.J Transl Med. 2014;12:29.

48. McAuley E, Jerome GJ, Marquez DX, Elavsky S, Blissmer B. Exercise selfefficacy in older adults: social, affective, and behavioral influences. Ann Behav Med. 2003;25(1):1-7.

49. Kangovi S, Asch DA. Behavioral Phenotyping in Health Promotion: Embracing or Avoiding Failure. JAMA. 2018;319(20):2075-2076.

50. Gommans LN, Hageman D, Jansen I, et al. Minimal correlation between physical exercise capacity and daily activity in patients with intermittent claudication. J Vasc Surg. 2016;63(4):983-989.

51. McDermott MM, Guralnik JM, Tian L, et al. Comparing 6-minute walk versus treadmill walking distance as outcomes in randomized trials of peripheral artery disease. J Vasc Surg. 2020;71(3):988-1001.

52. Fokkenrood HJ, Houterman S, Schep G, TeijinkJA, Scheltinga MR. Bicycle testing as an alternative diagnostic tool in patients suspected of intermittent claudication. Ann Vasc Surg. 2014;28(3):614-619.

53. Brostow DP, Petrik ML, Starosta AJ, Waldo SW. Depression in patients with peripheral arterial disease: A systematic review. Eur J Cardiovasc Nurs. 2017;16(3):181-193.

54. McDermott MM, Guralnik JM, Tian L, et al. Incidence and Prognostic Significance of Depressive Symptoms in Peripheral Artery Disease. J Am Heart Assoc. 2016;5(3):e002959.
55. Smolderen KG, Hoeks SE, Pedersen SS, van Domburg RT, de L, II, Poldermans D. Lower-leg symptoms in peripheral arterial disease are associated with anxiety, depression, and anhedonia. Vasc Med. 2009;14(4):297-304.

56. Piepoli MF, Hoes AW, Agewall S, et al. 2016 European Guidelines on cardiovascular disease prevention in clinical practice: The Sixth Joint Task Force of the European Society of Cardiology and Other Societies on Cardiovascular Disease Prevention in Clinical Practice (constituted by representatives of 10 societies and by invited experts)Developed with the special contribution of the European Association for Cardiovascular Prevention \& Rehabilitation (EACPR). Eur Heart $J$. 2016;37(29):2315-2381.

57. Smolderen $K G$, Aquarius $A E$, de Vries J, Smith OR, Hamming JF, Denollet J. Depressive symptoms in peripheral arterial disease: a follow-up study on prevalence, stability, and risk factors. J Affect Disord. 2008;110(1-2):27-35.

58. Sliwka A, Furgal M, Maga $P$, et al. The role of psychopathology in perceiving, reporting and treating intermittent claudication: a systematic review. Int Angiol. 2018;37(5):335-345.

59. Rejeski WJ, Tian L, Liao Y, McDermott MM. Social cognitive constructs and the promotion of physical activity in patients with peripheral artery disease. J Cardiopulm Rehabil Prev. 2008;28(1):65-72. 



\section{Chapter}

\section{Impactparagraaf}


Dit proefschrift gaat over de behandeling van 'etalagebenen' (medische term is 'claudicatio intermittens'), in dit proefschrift vaak afgekort tot IC. Patiënten met deze aandoening hebben pijn in de benen bij het lopen en zijn daardoor vaak beperkt in hun dagelijks functioneren. Deze klachten worden veroorzaakt door vernauwing van de beenslagaders ten gevolge van slagaderverkalking (atherosclerose). Dit zogenoemde 'perifeer arterieel vaatlijden' is een veelvoorkomende vaatziekte die veel overeenkomsten vertoont met andere hart- en vaatziekten zoals hart- en herseninfarcten. Het voornaamste behandeldoel op korte termijn is zo ver mogelijk klachtenvrij kunnen lopen. Voor de lange termijn is het belangrijk om het risico op andere hart- en vaatziekten te verlagen, aangezien het risico daarop bij IC sterk is verhoogd. De behandeling volgt een trapsgewijze volgorde, het zogenaamde 'stepped care model' (SCM). Hierin is gesuperviseerde looptherapie (GLT) de eerste keus behandeling en wordt een invasieve ingreep (een dotter of bypass operatie) pas overwogen als GLT onvoldoende effect heeft. Het hoofddoel van dit proefschrift is het verbeteren van de behandeling van etalagebenen door personalisatie, ofwel de inhoud afstemmen op het individu, met meer aandacht voor het verlagen van het risico op andere hart- en vaatziekten.

In dit proefschrift (hoofdstuk 3) wordt het resultaat van een samenwerking met het Nederlands Zorginstituut in het kader van het 'Zinnige Zorg' project beschreven. ${ }^{1}$ Een belangrijk doel van dit project is het voorkomen van onnodige ingrepen. Ons onderzoek toont aan dat naleving van de richtlijn de afgelopen jaren sterk is verbeterd. Waar in $201363 \%$ van de patiënten startte met GLT, was dit in 2017 gestegen tot $87 \%$. Hoewel geen onderwerp van studie in dit proefschrift, is het goed om hier te vermelden dat Nederland hierin een uitzonderlijke positie inneemt. In geen enkel ander land ter wereld wordt standaard GLT aangeboden. Niet uit onwil van dokters, maar simpelweg omdat het niet beschikbaar is. Althans niet in een landelijk dekkend, gestandaardiseerd concept waarin de kwaliteit van behandeling is geborgd.

In dit proefschrift wordt aangetoond dat de in Nederland gerealiseerde richtlijn naleving gepaard gaat met minder vaatingrepen. Vaatingrepen kunnen, hoewel zelden, gepaard gaan met complicaties, zoals bloedingen, hart- en of herseninfarcten en het verlies van een been. Na GLT had maar liefst $83 \%$ van de patiënten na 5 jaar nog steeds geen vaatingreep ondergaan. Wanneer daarentegen direct werd gekozen voor dotteren of opereren, bleek de kans op een tweede ingreep juist groter te zijn. Behalve dat deze bevinding duidt op minder onnodige ingrepen en de daarmee gepaard gaande risico's, zou dit ook tot een enorme kostenbesparing leiden. Hugo Fokkenrood, een van mijn voorgangers, berekende in een eerdere studie dat wanneer $80 \%$ van de patiënten volgens het SCM behandeld zou worden, ofwel zou starten met GLT, dit in Nederland een besparing van 33 miljoen euro per jaar zou opleveren. ${ }^{2}$

Een andere relevante bevinding van het onderzoek in hoofdstuk 3 was de stijging van het aantal GLT verwijzingen via de huisarts (zie tabel 1). Deze bevinding is 
bewust niet in de wetenschappelijke publicatie vermeld, omwille van relevantie en helderheid voor de internationale doelgroep, maar komt uiteraard wel terug in de projectrapporten. In het verleden kwamen alle patiënten met etalagebenen standaard bij de vaatchirurg terecht, terwijl de huisarts eigenlijk prima zelf de diagnose kan stellen en iemand mag verwijzen voor GLT. Dit laatste is efficiënter en meer kostenbewust dan ziekenhuiszorg en past binnen het streven 'de juiste zorg op de juiste plek'. De gevonden stijging is veelbelovend. Momenteel wordt gewerkt aan het zo goed mogelijk faciliteren van adequate diagnostiek via de huisarts zonder tussenkomst van een vaatchirurg.

Tabel 1. Verwijzingen voor gesuperviseerde looptherapie door huisartsen vs. vaatchirurgen

\begin{tabular}{llllll}
\hline & 2013 & 2014 & 2015 & $2016^{*}$ & 2017* \\
\hline Verwijzing door huisarts & 1858 & 1857 & 2116 & 2453 & 3686 \\
Verwijzing door vaatchirurg & 4890 & 5198 & 5532 & 5899 & 5987 \\
\% Verwijzingen door huisarts (/ totaal aantal) & $\mathbf{2 8 \%}$ & $\mathbf{2 6 \%}$ & $\mathbf{2 8 \%}$ & $\mathbf{2 9 \%}$ & $\mathbf{3 8 \%}$ \\
\hline
\end{tabular}

* Jaren 2016 en 2017 moeten proportioneel naar boven worden bijgesteld, omdat de data 90\% respectievelijk $80 \%$ compleet waren.

Ook hoofdstuk 4 en 5 sluiten aan bij het doel om onnodige operaties te voorkomen. Een veelgebruikt argument om direct te kiezen voor een vaatingreep in plaats van GLT is de locatie van de vaatvernauwing. Meerdere gecontroleerde studies ("randomised controlled trials", RCT) leverden echter geen wetenschappelijk bewijs voor dit argument. Er bleek behoefte te zijn aan een studie waarin de gebruikelijke zorg werd verleend zonder teveel aanpassingen in het kader van onderzoek. Aan de ELECT studie mochten vrijwel alle patiënten met etalagebenen meedoen, er waren geen uitgebreide (zogenaamde inclusie-) criteria, om zo dichtbij de dagelijkse praktijk te blijven. Bij elke patiënt werd de locatie van de vaatvernauwing vastgesteld en alle patiënten begonnen met GLT. Het bleek dat locatie van de vaatvernauwing géén invloed had op de resultaten van GLT. Dit zou dus niet langer een argument mogen zijn om af te wijken van de richtlijn. Naar verwachting draagt ook deze bevinding bij aan het voorkomen van onnodige vaatingrepen.

Een ander potentieel argument om af te wijken van de richtlijn hangt samen met psychologische factoren. Mogelijk zouden "negatieve" factoren, zoals bijvoorbeeld depressie en angst, een ongunstige invloed hebben op de resultaten van GLT. Hierover was nog relatief weinig bekend. Het onderzoek in hoofdstuk 6 laat zien dat dergelijke psychologische factoren geen invloed hebben op de effectiviteit van GLT. Dit betekent dus dat, mede op basis van de resultaten van dit proefschrift, er géén vaste patiëntkenmerken zijn die suggereren dat iemand met etalagebenen niet zou profiteren van GLT. 
Ook al wordt GLT als eerste keus behandeling aanbevolen door alle (inter) nationale richtlijnen, het percentage van $87 \%$ in Nederland blijkt toch uitzonderlijk hoog te zijn. Financiële vergoeding kan een drempel zijn voor het volgen van het SCM. Daarnaast is bewustwording belangrijk. Zowel de patiënt als verwijzend arts moeten gaan inzien dat GLT een volwaardige en effectieve behandeling is. Dit wordt in toenemende mate bevorderd door wetenschappelijke publicaties, vernieuwde (inter)nationale richtlijnen en door communicatie binnen de relevante patiënt- en specialistenorganisaties. Een derde belangrijke uitdaging is de organisatie van het SCM. In Nederland wordt GLT georganiseerd via Chronisch Zorgnet (voormalig ClaudicatioNet), een landelijk netwerk van gespecialiseerde fysiotherapeuten. Inmiddels wordt vanuit Chronisch ZorgNet met steeds meer landen, zoals lerland, Portugal en België, relevante kennis en expertise gedeeld om ook internationaal de richtlijn navolging te helpen verbeteren. Hierin worden ook de resultaten van dit proefschrift meegenomen.

GLT, zoals de naam al aangeeft, bestaat voornamelijk uit looptherapie. Aangezien de klachten optreden tijdens het lopen lijkt dit logisch. Echter, soms doet lopen teveel pijn of heeft een patiënt andere aandoeningen waardoor lopen beperkt wordt. In die gevallen kunnen alternatieve trainingsvormen interessant zijn. Bovendien kan een uitgebreider en/of gevarieerder traject zorgen voor betere aansluiting op de internationale beweegrichtlijnen. In hoofdstuk 7 laat een review zien dat alternatieven, zoals Nordic walking, fietsen, weerstandtraining en combinaties daarvan, vergelijkbare resultaten kunnen geven als GLT. Fysiotherapeuten aangesloten bij Chronisch ZorgNet zullen dit opnemen in hun behandelprogramma's.

Samen met het vergroten van de klachtenvrije loopafstand is het vóórkomen van nieuwe hart- en vaatziekten het belangrijkste behandeldoel bij etalagebenen. In de praktijk betekende dit voornamelijk dat de huisarts of vaatchirurg medicatie voorschrijft en dat men enkele adviezen krijgt over gezonde leefstijl. Er wordt steeds meer duidelijk over de impact van leefstijl op bepaalde risicofactoren en hoe je dit het beste kunt beïnvloeden. Beweging blijkt hierin een bijzondere rol te spelen en is dus een belangrijk aangrijpingspunt voor verbetering. Er was niet eerder onderzocht of GLT, wat ook een vorm van beweging is, invloed heeft op die risicofactoren bij patiënten met etalagebenen. In hoofdstuk 2 zijn analyses uitgevoerd met bestaande studies, waaruit blijkt dat GLT in ieder geval positieve invloed heeft op de bloeddruk en het cholesterol. De verwachting is dat deze effecten vergroot kunnen worden door het GLT traject te intensiveren en met name nog meer te richten op leefstijl.

De inzichten van dit proefschrift hebben er mede toe geleid dat GLT in toenemende mate wordt beschouwd als een duurzame leefstijlinterventie i.p.v. een korte termijn oplossing die slechts is gericht op het verbeteren van de klachten. Binnen Chronisch ZorgNet zijn de bevindingen van dit proefschrift opgenomen in de basisopleiding en nascholingen voor aangesloten fysiotherapeuten. Naast het vergroten van kennis, worden ook meer handvatten aangereikt om de leefstijlbegeleiding te intensiveren. Zo wordt naast de basis cursus 'motivational 
interviewing' een verdiepingscursus 'motivational interviewing' toegevoegd aan de verplichte nascholing van aangesloten fysiotherapeuten. Tevens is in samenwerking met het Trimbos Instituut een stoppen met roken cursus opgestart, zodat inmiddels meer dan 400 fysiotherapeuten geregistreerd rookstop coach konden worden.

Alhoewel bepaalde psychologische kenmerken geen invloed hadden op de uitkomsten van looptherapie, viel wel op dat angst- en depressieve klachten relatief vaak voorkomen bij patiënten met etalagebenen. Daarom wordt tijdens de basisopleiding en nascholing ook steeds meer aandacht besteed aan psychosociale factoren. Het doel is dat dit onderwerp actief bespreekbaar wordt gemaakt door de fysiotherapeut, zodat indien nodig adequate ondersteuning kan worden geregeld voor de patiënt.

Alle artikelen uit dit proefschrift zijn gepubliceerd in wetenschappelijke tijdschriften. De resultaten zijn tevens gecommuniceerd aan patiënten d.m.v. nieuwsberichten en informatieve pagina's op de website. Bovendien vormt dit promotieonderzoek de basis voor het hoofdstuk 'Beweging' in het eerste handboek voor leefstijlgeneeskunde voor artsen en paramedici dat in 2020 is verschenen. ${ }^{3}$

\section{REFERENTIES}

1. Dutch National Healthcare Institute. Room for improvement analysis: Peripheral Artery Disease 2016. Available from: https://english.zorginstituutnederland.nl/publications/ reports/2018/05/30/room-for-improvement-analysis-claudication-intermittent-zinnigezorg [Accessed June 2020].

2. Fokkenrood HJ, Scheltinga MR, Koelemay MJ, et al. Significant savings with a stepped care model for treatment of patients with intermittent claudication. Eur J Vasc Endovasc Surg. 2014;48(4):423-429.

3. Teijink, J. \& Jansen, S. Hoofdstuk 12: Beweging. De Vries, M., \& de Weijer, T. (Red.) Handboek Leefstijlgeneeskunde (pp. 221-237). Houten, Nederland: Bohn Stafleu van Loghum. 2020 https://doi.org/10.1007/9789036823241. 



\section{Chapter}

\section{Dankwoord}

\section{Lijst van publicaties}

Curriculum Vitae 


\section{DANKWOORD}

Het dankwoord, vreemd genoeg is dit het meest gelezen hoofdstuk van een proefschrift. Hoewel ik zelf erg graag lees, heb ik besloten om dit hoofdstuk kort te houden. Want iemand bedanken doe ik liever persoonlijk.

Dit proefschrift vormt samen met mijn verdediging de afsluiting van mijn promotietraject. Een periode met ups en downs, nieuwe ervaringen en beproevingen. Dit proefschrift was niet tot stand gekomen zonder de medewerking van patiënten, nationale en internationale samenwerkingen en steun van vrienden en familie. Bij deze wil ik iedereen bedanken.

Prof. dr. J.A.W. Teijink, beste Joep. Als semiarts in het Catharina zei ik nog dat ik nooit fulltime wilde promoveren en al zeker niet bij de vaatchirurgie. Om mijn kansen op een opleidingsplek te vergroten ging ik toch op zoek en kwam ik bij jou terecht. Met jouw ongekende enthousiasme en gedrevenheid had je mij overtuigd en zouden we de wetenschappelijke stappen richting Chronisch ZorgNet gaan zetten. Het is bewonderenswaardig hoe jij je inzet voor de conservatieve behandeling van patiënten, niet alleen met claudicatio maar ook met andere chronische ziekten. Zoals ik tijdens mijn promotietijd de "breedte" opzocht, besloot ik dit ook in de toekomst door te zetten. Toen ik je vertelde dat ik bij de luchtmacht wilde solliciteren reageerde je heel enthousiast. Ik merkte wel dat je een beetje zenuwachtig werd of dat boekje nog wel zo snel af zou komen als ik eenmaal weg was... Zoals je ziet heb ik me aan mijn woord gehouden!

Dr. M.R.M. Scheltinga, beste Marc. Het was heel fijn hoe je af en toe uitgebreid de tijd nam om eens alles door te spreken, inclusief vakantieplannen en -foto's, precies wat je nodig hebt van een copromotor. Je praat vol passie over onderzoek en de vele ideeën die je hebt, dat werkt aanstekelijk. De snelheid waarmee jij op mails reageert en teksten reviseert deed me steeds versteld staan. Gelukkig kwamen de teksten steeds minder rood terug, ik zal de "slet" methode nooit vergeten. Bedankt dat ik zoveel van jou heb mogen leren.

Dr. E.V. Rouwet, beste Ellen. Hoeveel promovendi kunnen zeggen dat ze samen met hun copromotor een epidemiologie cursus hebben gevolgd?! Het was een leerzame en vooral ook leuke ervaring om samen met jou en Sanne de mindfulness RCT te ontwerpen tijdens de NIHES cursus. Je bent als betrokken copromotor oprecht geïnteresseerd. We hebben goede gesprekken gehad over de chirurgie en het verbaasde je helemaal niet dat ik een ander pad koos. Zelf ben je ook een nieuwe uitdaging aangegaan, met jouw centrum voor leefstijlgeneeskunde ga je ongetwijfeld veel mensen helpen!

Medeauteurs van diverse artikelen, bedankt voor jullie bijdrage aan het tot stand brengen van een aantal mooie publicaties. Een aantal mensen wil ik in het bijzonder bedanken. Anco Vahl, het was een hele fijne ervaring om met jou samen te schrijven. Je bent zeer betrokken, ook op detailniveau. Bedankt voor je kritische blik en vele ideeën. Sanne Hoeks, dankzij jou heb ik ontzettend veel geleerd van complexe 
statistische analysen. Ik heb regelmatig $\mathrm{R}$ afgesloten met een warrig hoofd van alle codes, maar inmiddels weet ik er aardig mijn weg in te vinden. Ivan Nyklicek, een stel "chirurgen" die zich gaan verdiepen in de psychosociale wetenschap, zonder jouw waardevolle inzichten was dat niet gelukt! Bert van Nistelrooij, de data van het zorginstituut mag het gebouw niet uit, maar we wisten de afstand goed te overbruggen door regelmatig een dagje samen op het zorginstituut te werken en veel telefonisch overleg. Bedankt voor die fijne samenwerking. Gert-Jan Lauret, bedankt dat ik de Cochrane review van jou als 1e auteur mocht overnemen voor deze update. Desondanks bleef je zeer betrokken. Ukay Abaraogu, you were the one to notice that the Cochrane Review needed an update and that is how our cooperation came about. Thank you for updating the review together with me.

Uiteraard wil ik ook alle patiënten bedanken die direct of indirect hebben bijgedragen aan dit proefschrift. In het bijzonder de deelnemers aan de ELECT Registry, die de vele vragenlijsten met allerlei 'rare vragen' eerlijk hebben ingevuld. Ook het telefonisch contact met honderden claudicatio patiënten heeft me veel inzichten opgeleverd.

Leden van de beoordelingscommissie, hartelijk dank voor de beoordeling van mijn proefschrift.

Collega-onderzoekers van 'de zolder'. Naast de ups en downs van promoveren was er tijd voor zolderuitjes, zolderpresentaties (streng wetenschappelijk beoordeeld) en discodinsdag/donderdag/vrijdag. Hoeveel onderzoekers kunnen zeggen dat ze met regelmaat werden toegezongen door hun collega's? Ik zal niet vergeten hoe jullie het lied 'Sunny, yesterday my life was filled with rain...' (natuurlijk uitgesproken als 'Sannie') vol enthousiasme inbrachten.

Als onderzoeker bij Joep wordt je automatisch onderdeel van het ClaudicatioNet team (inmiddels Chronisch ZorgNet). Wat een gedreven en gezellige club mensen! Diners, uitjes, de volleybal wedstrijd (die wij natuurlijk gewonnen hebben). En natuurlijk de SMR cursus, waarvoor Yvonne en ik het land door mochten trekken als semiprofessionele cameraploeg. Ook bedankt Nicole voor het helpen coördineren van dit promotietraject.

Last but certainly not least, ik wil graag mijn familie en vrienden bedanken voor alle steun en welkome afleiding. Ook al was het voor sommigen van jullie lastig te volgen wat ik nou eigenlijk precies deed, ik kon altijd op jullie interesse en steun rekenen. Lieve Faizah en Laura, bedankt dat jullie mij als paranimfen willen helpen met de laatste loodjes van dit traject. 


\section{LIJST VAN PUBLICATIES}

Jansen SC, Groeneveld-Haenen CP, Klinkhamer PJ, Roumen RM. Spontaneous regression and recurrence of stage III Merkel cell carcinoma. BMJ Case reports. Feb 25;2015.

SCP Jansen, J Bransen, G van Montfort, AT Besselaar, AH van der Veen. Should the Extended Lateral Approach Remain Part of Standard Treatment in Displaced Intra-articular Calcaneal Fractures? Journal of Foot \& Ankle Surgery. 2018 Nov - Dec;57(6):1120-1124

Jansen SCP, Hoorweg BBN, Hoeks SE, van den Houten MML, Scheltinga MRM, Teijink JAW, Rouwet EV. A systematic review and meta-analysis of the effects of supervised exercise therapy on modifiable cardiovascular risk factors in intermittent claudication. J Vasc Surg. 2019 Apr;69(4):1293-1308.e2

van den Houten MML, Jansen SCP, Sinnige A, van der Laan L, Vriens PWHE, Willigendael EM, Lardenoije JWHP, Elshof JWM, van Hattum ES, Lijkwan MA, Nyklíček I, Rouwet EV, Koelemay MJW, Scheltinga MRM, Teijink JAW. Protocol for a prospective, longitudinal cohort study on the effect of arterial disease level on the outcomes of supervised exercise in intermittent claudication: the ELECT Registry. BMJ Open 2019;9:e025419

van den Houten MML, Jansen SCP, van der Laan L, Vriens PWHE, Willigendael EM, Koelemay MJW, Scheltinga MRM, Teijink JAW, ELECT Study Group. The effect of arterial disease level on outcomes of supervised exercise therapy for intermittent claudication: a prospective cohort study. Ann Surg. $2020 \mathrm{Jul} 24$. Online ahead of print

Jansen SCP, Abaraogu UO, Lauret GJ, Fakhry F, Fokkenrood HJP, Teijink JAW. Modes of exercise training for intermittent claudication. Cochrane Database Syst Rev. 2020 Aug 20;8:CD009638

Jansen SCP, van Nistelrooij LPJ, Scheltinga MRM, Rouwet EV, Teijink JAW, Vahl AC. Successful implementation of the exercise first approach for intermittent claudication in the Netherlands is associated with few lower limb revascularisations. Eur J Vasc Endovasc Surg. 2020 Dec;60(6):881-887

Jansen SCP, Hoeks SE, Nyklíček I, Scheltinga MRM, TeijinkJAW, Rouwet EV. Supervised exercise therapy is effective for patients with intermittent claudication regardless of psychological constructs. EurJ Vasc Endovasc Surg. 2021 Dec 6;510785884(21)00811-X. Online ahead of print. 


\section{Overig}

Handreiking Technologische Innovatie in de vervolgopleiding (november 2018). Werkgroep: dr. Irma Scholten, drs. Auk Dijkstra, dr. Bram van Rhijn, dr. Hein Handgraaf, dr. Julia D'Hooghe, drs. Sandra Jansen, mr. dr. Laura Kienhorst, Abel Swaan MSc, Juliette Velu MSc. Dit visiedocument is opgesteld door De Jonge Specialist in samenwerking met de Federatie Medisch Specialisten. Zie https:// innovatie.dejongespecialist.nl/ voor e-PDF.

Teijink, J. \& Jansen, S. Hoofdstuk 12: Beweging. De Vries, M., \& de Weijer, T. (Red.) Handboek Leefstijlgeneeskunde (pp. 221-237). Houten, Nederland: Bohn Stafleu van Loghum. 2020 


\section{CURRICULUM VITAE}

Sandra Jansen werd geboren op 19 februari 1992 in Veldhoven. Na het afronden van het Gymnasium startte ze in 2010 met de studie Geneeskunde aan de Universiteit Maastricht. In juni 2016 behaalde ze haar artsexamen (cum laude) en begon aansluitend als ANIOS heelkunde in het Jeroen Bosch Ziekenhuis te Den Bosch. Na deze eerste ervaring met "echt dokteren" besloot ze ruim een jaar later fulltime promotieonderzoek op te starten onder leiding van prof. dr. Teijink (Vaatchirurgie, Catharina Ziekenhuis te Eindhoven).

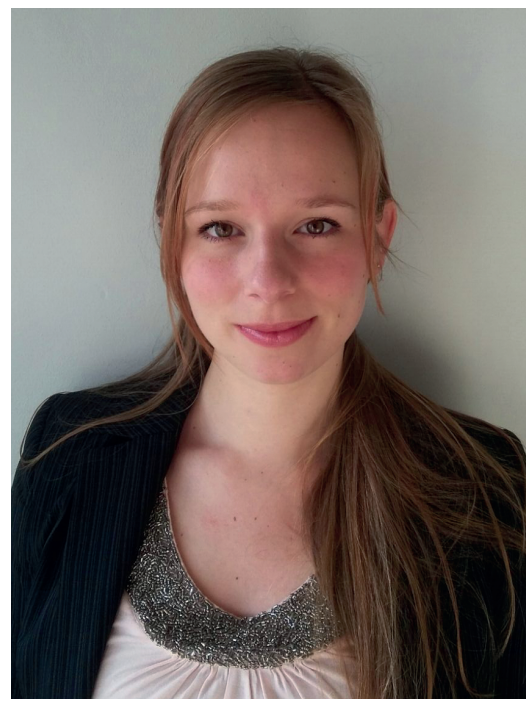

Naast haar onderzoek naar het optimaliseren van de conservatieve behandeling van claudicatio intermittens heeft zij zich binnen ClaudicatioNet voornamelijk ingezet voor de uitbreiding naar Chronisch ZorgNet. Dit deed zij onder andere door het leveren van wetenschappelijke onderbouwing, het geven van cursussen aan fysiotherapeuten en het mede ontwikkelen van de cursus rookstop coaching voor fysiotherapeuten in samenwerking met het Trimbos Instituut. Om dit zo goed mogelijk te kunnen doen heeft zij de Basistraining Motivational Interviewing (Academie voor Motivatie en Gedragsverandering) en de Summer Course Health Communication and Health Promotion (CAPHRI, Maastricht University) voltooid.

Tevens heeft zij de cursus Advanced Topics in Clinical Trials (NIHES, Erasmus MC Rotterdam) succesvol afgerond, waarna zij samen met o.a. copromotor Ellen Rouwet een multicenter RCT heeft opgezet naar de effectiviteit van mindfulness-based stress reduction therapie op het verbeteren van de kwaliteit van leven bij patiënten met atherosclerotische hart- en vaatzieken (coronair vaatlijden, ischemisch hartfalen en perifeer arterieel vaatlijden). Deze "Mind Our Heart Study" wordt momenteel opgestart in het Catharina Ziekenhuis.

Uiteindelijk heeft ze tijdens haar promotieonderzoek besloten om een andere professionele richting te kiezen. In maart 2020 is zij bevorderd tot kapitein arts na de 10 weken durende specialisten opleiding aan de Koninklijke Militaire Academie te Breda. Momenteel is zij in opleiding tot algemeen militair arts. Deze opleiding verwacht ze in april 2022 af te ronden om vervolgens werkzaam te zijn in de operationele gezondheidszorg van de Koninklijke Luchtmacht. 
